University of Louisville

ThinkIR: The University of Louisville's Institutional Repository

Electronic Theses and Dissertations

$12-2016$

\title{
Computational methods for the analysis of functional 4D-CT chest images.
}

Ahmed Soliman Naeem Soliman

University of Louisville

Follow this and additional works at: https://ir.library.louisville.edu/etd

Part of the Electrical and Computer Engineering Commons

\section{Recommended Citation}

Soliman, Ahmed Soliman Naeem, "Computational methods for the analysis of functional 4D-CT chest images." (2016). Electronic Theses and Dissertations. Paper 2567.

https://doi.org/10.18297/etd/2567

This Doctoral Dissertation is brought to you for free and open access by ThinkIR: The University of Louisville's Institutional Repository. It has been accepted for inclusion in Electronic Theses and Dissertations by an authorized administrator of ThinkIR: The University of Louisville's Institutional Repository. This title appears here courtesy of the author, who has retained all other copyrights. For more information, please contact thinkir@louisville.edu. 


\title{
COMPUTATIONAL METHODS FOR THE ANALYSIS OF FUNCTIONAL 4D-CT CHEST IMAGES
}

\author{
By \\ Ahmed Soliman Naeem Soliman \\ M.Sc., CES, Mansoura University, Mansoura 35516, Egypt, 2010
}

\begin{abstract}
A Dissertation
Submitted to the Faculty of the

J. B. Speed School of the University of Louisville in Partial Fulfillment of the Requirements for the Degree of

Doctor of Philosophy in Electrical Engineering

Department of Electrical and Computer Engineering

University of Louisville

Louisville, Kentucky
\end{abstract}

December 2016 



\title{
COMPUTATIONAL METHODS FOR THE ANALYSIS OF FUNCTIONAL 4D-CT CHEST IMAGES
}

\author{
By \\ Ahmed Soliman Naeem Soliman \\ M.Sc., CES, Mansoura University, Mansoura 35516, Egypt, 2010
}

A Dissertation Approved on

November 15th, 2016

by the Following Dissertation Committee:

Ayman El-Baz, Ph.D., Dissertation Director

Jacek Zurada, Ph.D., Co-advisor

Cindy Harnett, Ph.D.

Olfa Nasraoui, Ph.D.

Manuel Casanova, M.D. 


\section{DEDICATION}

This dissertation is dedicated to my father's soul. 


\section{ACKNOWLEDGMENTS}

In the name of Allah the most merciful, the most compassionate. All deepest thanks are due to Almighty Allah for the uncountable gifts given to me.

I would like to thank my dissertation advisor, Dr. Ayman El-Baz, my dissertation advisor, for his continuous encouragement, guidance, and support over the course of my Ph.D. study. I thank him for giving me the opportunity to work in his research group and providing me the opportunity to work on promising applications and meet interesting people. Without his expertise, guidance, and commitment, my research would not have been possible. I truly appreciate his optimism whenever things looked impossible.

I also express my deepest gratitude to Dr. Jacek Zurada, my dissertation co-advisor, and Dr. Cindy Harnett, Dr. Olfa Nasraoui, and Dr. Manuel Casanova for being on my dissertation committee with enthusiasm and taking interest in my research in the midst of many other responsibilities and commitments.

I also have to thank Dr. Georgy Gimel' farb for his useful discussions and valuable comments and feedback. He has never hesitated to share his experience in Markov-Gibbs random field and the field of image processing. I also would like to thank Dr. Mohamed Abou El-Ghar of the radiology department, Urology and Nephrology Center, University of Mansoura, Egypt. They spent much of their valuable time providing advices for my work in the analysis of CT images and commenting on its quality.

I also express my deepest thanks to all members of the research group in the BioImaging Laboratory at the University of Louisville. Last but not least, I would like to sincerely thank all my family members. 


\title{
ABSTRACT \\ COMPUTATIONAL METHODS FOR THE ANALYSIS OF FUNCTIONAL 4D-CT CHEST IMAGES
}

\author{
Ahmed Soliman Naeem Soliman
}

November 15th, 2016

\begin{abstract}
Medical imaging is an important emerging technology that has been intensively used in the last few decades for disease diagnosis and monitoring as well as for the assessment of treatment effectiveness. Medical images provide a very large amount of valuable information that is too huge to be exploited by radiologists and physicians. Therefore, the design of computer-aided diagnostic (CAD) system, which can be used as an assistive tool for the medical community, is of a great importance. This dissertation deals with the development of a complete CAD system for lung cancer patients, which remains the leading cause of cancer-related death in the USA. In 2014, there were approximately 224,210 new cases of lung cancer and 159,260 related deaths. The process begins with the detection of lung cancer which is detected through the diagnosis of lung nodules (a manifestation of lung cancer). These nodules are approximately spherical regions of primarily high density tissue that are visible in computed tomography (CT) images of the lung. The treatment of these lung cancer nodules is complex, nearly $70 \%$ of lung cancer patients require radiation therapy as part of their treatment. Radiation-induced lung injury is a limiting toxicity that may decrease cure rates and increase morbidity and mortality treatment. By finding ways to accurately detect, at early stage, and hence prevent lung injury, it will have significant positive consequences for lung cancer patients.
\end{abstract}


The ultimate goal of this dissertation is to develop a clinically usable CAD system that can improve the sensitivity and specificity of early detection of radiation-induced lung injury based on the hypotheses that radiated lung tissues may get affected and suffer decrease of their functionality as a side effect of radiation therapy treatment. These hypotheses have been validated by demonstrating that automatic segmentation of the lung regions and registration of consecutive respiratory phases to estimate their elasticity, ventilation, and texture features to provide discriminatory descriptors that can be used for early detection of radiation-induced lung injury. The proposed methodologies will lead to novel indexes for distinguishing normal/healthy and injured lung tissues in clinical decisionmaking. To achieve this goal, a CAD system for accurate detection of radiation-induced lung injury that requires three basic components has been developed. These components are the lung fields segmentation, lung registration, and features extraction and tissue classification.

This dissertation starts with an exploration of the available medical imaging modalities to present the importance of medical imaging in today's clinical applications. Secondly, the methodologies, challenges, and limitations of recent CAD systems for lung cancer detection are covered. This is followed by introducing an accurate segmentation methodology of the lung parenchyma with the focus of pathological lungs to extract the volume of interest (VOI) to be analyzed for potential existence of lung injuries stemmed from the radiation therapy. After the segmentation of the VOI, a lung registration framework is introduced to perform a crucial and important step that ensures the co-alignment of the intra-patient scans. This step eliminates the effects of orientation differences, motion, breathing, heart beats, and differences in scanning parameters to be able to accurately extracts the functionality features for the lung fields. The developed registration framework also helps in the evaluation and gated control of the radiotherapy through the motion estimation analysis before and after the therapy dose. Finally, the radiation-induced lung injury is introduced, which combines the previous two medical image processing and analysis steps with the 
features estimation and classification step. This framework estimates and combines both texture and functional features. The texture features are modeled using the novel $7^{\text {th }}$-order Markov Gibbs random field (MGRF) model that has the ability to accurately models the texture of healthy and injured lung tissues through simultaneously accounting for both vertical and horizontal relative dependencies between voxel-wise signals. While the functionality features calculations are based on the calculated deformation fields, obtained from the 4D-CT lung registration, that maps lung voxels between successive CT scans in the respiratory cycle. These functionality features describe the ventilation, the air flow rate, of the lung tissues using the Jacobian of the deformation field and the tissues' elasticity using the strain components calculated from the gradient of the deformation field. Finally, these features are combined in the classification model to detect the injured parts of the lung at an early stage and enables an earlier intervention. 


\section{TABLE OF CONTENTS}

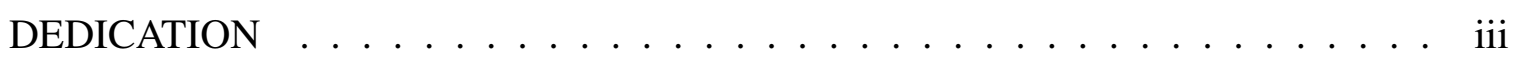

ACKNOWLEDGMENTS .................. iv

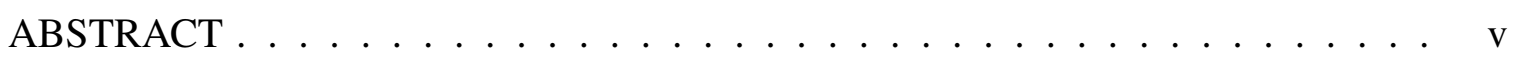

LIST OF TABLES $\ldots \ldots \ldots \ldots \ldots \ldots \ldots \ldots \ldots$ xiii

LIST OF FIGURES $\ldots \ldots \ldots \ldots \ldots \ldots \ldots \ldots$ xv

LIST OF ALGORITHMS $\ldots \ldots \ldots \ldots \ldots \ldots \ldots \ldots \ldots$ xx

\section{CHAPTER}

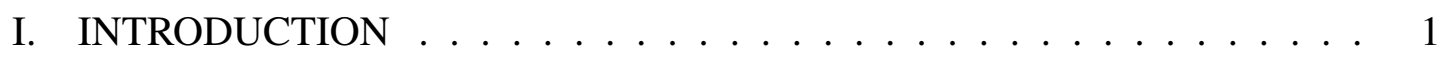

A. Computed tomography $(\mathrm{CT}) \ldots \ldots \ldots \ldots \ldots \ldots$

1. Structural CT .................. 3

2. Contrast-enhanced CT (CE-CT) . . . . . . . . . . 4

3. CT angiography $(\mathrm{CTA}) \ldots \ldots \ldots \ldots \ldots \ldots$

4. Microtomography (Micro-CT) . . . . . . . . . . . . 6

5. Dynamic CT imaging (4D-CT) $\ldots \ldots \ldots \ldots \ldots$

6. CT imaging: Pros and cons $\ldots \ldots \ldots \ldots \ldots$

B. Nuclear medical imaging (nuclide imaging) $\ldots \ldots \ldots \ldots$

C. Computed tomography Applications in different diseases . . . . . . 12

D. Dissertation organization $\ldots \ldots \ldots \ldots \ldots \ldots \ldots$ 


\section{COMPUTER-AIDED DIAGNOSIS SYSTEMS FOR LUNG CANCER: A}

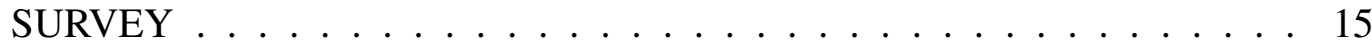

A. Introduction . . . . . . . . . . . . . . . 15

B. Lung Segmentation . . . . . . . . . . . . . . . . . . . . 17

C. Detection of lung nodules . . . . . . . . . . . . . . . . . 24

D. Lung Nodule Segmentation . . . . . . . . . . . . . . . . 33

1. Clinical Applications . . . . . . . . . . . . . . 33

2. CT Nodules Segmentation Techniques . . . . . . . . . . 34

a. Thresholding ................... 35

b. Mathematical morphology ... . . . . . . . . . 35

c. Region growing ... . . . . . . . . . . 35

d. Deformable model . . . . . . . . . . . . . . 36

e. Dynamic programming ............. 36

f. Spherical/ellipsoidal model fitting . . . . . . . . . . 37

g. Probabilistic classification . . . . . . . . . . 38

h. Discriminative classification ............ . 38

i. Mean shift .................. . . 39

j. Graph-cuts (GCs) and Watersheds (WSs) . . . . . . . . . . 39

3. PET Segmentation Techniques . . . . . . . . . . . . . 45

4. Nodule Segmentation Challenges . . . . . . . . . . . . 51

a. Nodule Types . . . . . . . . . . . . . . . . . . 51

b. Automation .................... 55

c. Robustness .................... . . 56

d. Validation ................... 58 
E. Diagnosis of Lung Nodules _ . . . . . . . . . . . . . . 60

1. Diagnosis of lung nodules based on growth rate . . . . . . . 60

a. Technical Approaches . . . . . . . . . . . . . 62

2. Diagnosis of lung nodules based on shape and appearance . . . 68

3. PET/CT Nodule Diagnosis . . . . . . . . . . . . . . 78

F. Summary and Discussion . . . . . . . . . . . . . . 80

1. Research Challenges. . . . . . . . . . . . . . . . . 81

\section{SEGMENTATION OF PATHOLOGICAL LUNGS USING ADAPTIVE SHAPE} GUIDED BY CT IMAGES APPEARANCE $\ldots \ldots \ldots \ldots$

A. Introduction . . . . . . . . . . . . . . . . . . 84

B. Methods . . . . . . . . . . . . . . . . . 88

1. Adaptive probabilistic shape prior . . . . . . . . . . . . . 89

2. Gaussian scale space (GSS) smoothing . . . . . . . . . 95

3. First-order appearance (intensity) modeling . . . . . . . . . . 97

4. Modeling pairwise spatial interactions of lung labels . . . . . . 101

5. Joint MGRF model of 3D chest CT images . . . . . . . . . . 104

6. Classification using majority voting . . . . . . . . . . . . . 104

C. Performance Evaluation Metrics . . . . . . . . . . . . . . . . . 104

1. Dice Similarity Coefficient (DSC) . . . . . . . . . . . 105

2. overlap measure . . . . . . . . . . . . . . 106

3. percentage volume difference $(\mathrm{PVD}) \ldots \ldots$

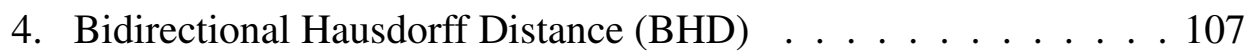

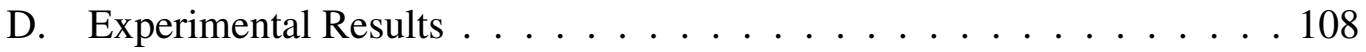

1. Evaluation using UoLDB. . . . . . . . . . . . . . . 108 
2. Evaluation using VESSEL12 database. . . . . . . . . . . . 114

3. Evaluation using LOLA11 database. . . . . . . . . . . . 115

E. Summary and Discussion . . . . . . . . . . . . . . . . 122

IV. AN ISO-SURFACES BASED LOCAL DEFORMATION HANDLING FRAMEWORK OF LUNG TISSUES . . . . . . . . . . . . . . . . . 125

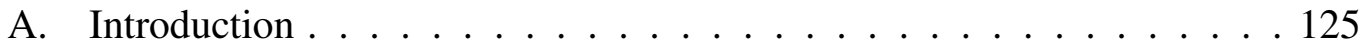

B. Methods . . . . . . . . . . . . . . . . . . . . . 127

1. Global Alignment . . . . . . . . . . . . . . . . . . 127

2. Local Alignment . . . . . . . . . . . . . . . . . . . 129

3. Correspondences Refinement . . . . . . . . . . . . . 132

C. Experimental Results and Validation . . . . . . . . . . . 135

1. Qualitative Evaluation . . . . . . . . . . . 138

2. Quantitative Evaluation . . . . . . . . . . 138

a. In-vivo Data Validation . . . . . . . . . . . . . 138

b. Simulated Data Validation . . . . . . . . . . . . 139

D. Summary . . . . . . . . . . . . . . . . . . . . 140

V. COMPUTER AIDED DIAGNOSIS SYSTEM FOR ACCURATE DETECTION OF RADIATION-INDUCED LUNG INJURY . . . . . . . . . . . 143

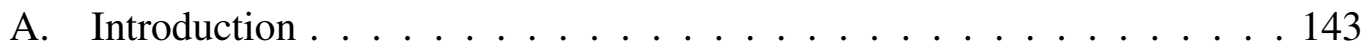

B. Methods . . . . . . . . . . . . . . . . . . . . 146

1. Deformable Image Registration . . . . . . . . . . . . . 147

2. 4D-CT Lung Segmentation . . . . . . . . . . . . . . 149

3. RILI detection Using Texture and Functionality Features . . . . 151

a. The $7^{t h}$-order textural feature: . . . . . . . . . . 151 
b. Appearance differences in follow-up scans . . . . . . . . . 154

c. Functionality features . . . . . . . . . . . 155

4. Tissue Classification . . . . . . . . . . . . . 157

C. Experimental Results and Conclusions . . . . . . . . . . . . . . . . 159

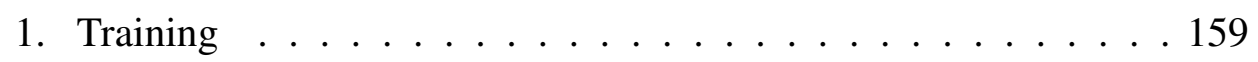

2. Data Augmentation ................ . . 160

3. Scale Generation . . . . . . . . . . . . . . . 160

4. Results ...................... 161

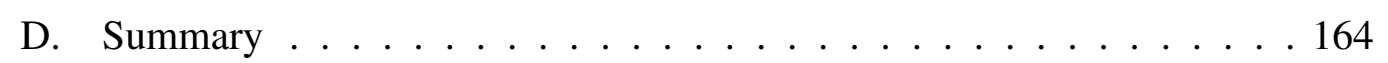

VI. CONCLUSIONS AND FUTURE WORK . . . . . . . . . . . . . 166

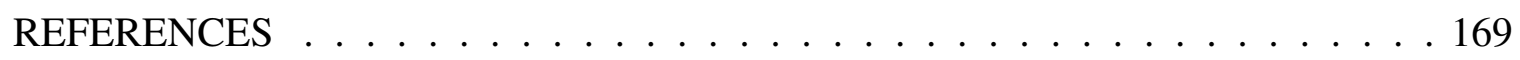

CURRICULUM VITAE . . . . . . . . . . . . . . . . . 211 


\section{LIST OF TABLES}

TABLE.

PAGE

1. Typical radionuclides for nuclear medical imaging. . . . . . . . . . . . . . 9

2. Review of current lung segmentation approaches ordered by their publication

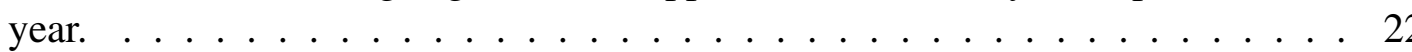

3. Classification component in CADe schemes. Studies are ordered by their pub-

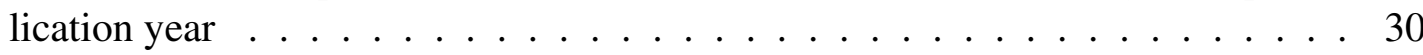

4. Studies on volumetric nodule segmentation reported from 1998 to 2011. Studies are ordered by their publication year. The purpose, type, and basic idea of each reported segmentation method are briefly described. Data and method used for validation of proposed methods are also described. CCL: connected component labeling; DP: dynamic programming; ECLAP: early lung cancer action program; EM: expectation-maximization; GGO: ground-glass opacity (non-solid and part-solid) nodules; GMM: Gaussian mixture model; GT: ground-truth; kNN: k-nearest neighbor; LDA: linear discriminant analysis; LS: level-sets; MAP: maximum a priori; MO: morphological operations; MRF: Markov random fields; RMS: root mean square; SMDC: selective marking and depth constrained;

5. Summary of lung nodule segmentation approaches from PET images. For each study, the table summarizes the number of the patients enrolled in the study and the type of the nodule delineation approach with respect to the methodology, the approach dimension, and the automation level. . . . . . . . . . . . . . 48

6. Assessing effect of PET/CT on GTV. For each study, the number of patients, the PET/CT fusion method, and the increase and decrease in the GTV as a percentage of the total number of the study cases is reported. . . . . . . . 50

7. Growth rate based methodologies for following up pulmonary nodules. . . . . . 65

8. Classification between malignant and benign nodules based on shape and ap-

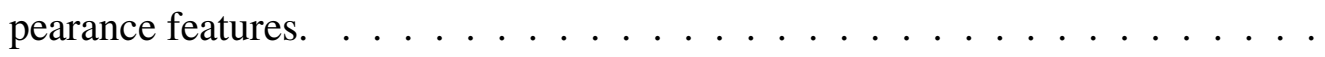


9. Evaluation of nodule malignancy in PET . . . . . . . . . . . . . . . . . 79

10. Evaluation of nodule malignancy in fused PET/CT . . . . . . . . . . . . 80

11. DSC, BHD, and PVD accuracy ( mean $_{ \pm \text {standar deviation }}$ ) of different components (feature groups FG) of the proposed framework on the UoLDB in terms of the: FG1 (intensities and spatial interactions), FG2 (combined original / GSSfiltered intensities and spatial interactions), FG3 (FG2 + adaptive shape priors). . 113

12. DSC, BHD, and PVD accuracy $\left(\right.$ mean $\left._{ \pm \text {standar deviation }}\right)$ of the proposed method and FC segmentation [1] on the UoLDB and VESSEL [2] databases. . . . . . 113

13 Right and left lung overlap scores for each subject in LOLA11 challenge . . . . 117

14. Mean overlap of the proposed segmentation method with the ground truth for the entire LOLA 11 database of 55 chest CT images vs. the $2^{\text {nd }}-5^{\text {th }}$-rank LOLA11 competitors (these accuracies were provided by the LOLA11 organizers [3]). . . 120

15. Comparison of registration accuracy for the 4D-CT DIR-lab data (All units are in $\mathrm{mm}) . \ldots \ldots \ldots \ldots$. . . . . . . . . . . . . . . . . . . . . . . . . . . . . . . . .

16. Registration accuracy for simulated displacements (All units are in mm). . . . . 141

17. Classification Accuracy using Cross Validation for deifferent feature groups: FG1 (Texture features), FG2 (Functionality features), and FG3 (combination of

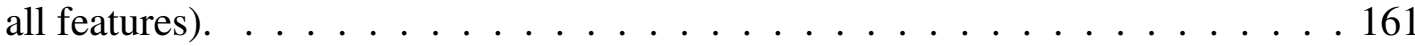




\section{LIST OF FIGURES}

FIGURE.

PAGE

1. Most popular types of medical images. . . . . . . . . . . . . . 2

2. Axial brain (a), lung (b), and heart (c) CT scans. . . . . . . . . . . . . . . 4

3. Abdominal DCE-CT scans before (a); $90 \mathrm{sec}$ after, and $180 \mathrm{sec}$ after administering the contrast agent. . . . . . . . . . . . . . . 4

4. Brain CTA image. . . . . . . . . . . . . . . . 6

5. Nuclear medical imaging. . . . . . . . . . . . . . . . . 8

6. A typical computer-aided diagnosis for lung cancer. The input of the CAD system is the medical images obtained using the appropriate modality. A lung segmentation step is used to reduce the search space for lung nodules. Nodule detection is used to identify the lung nodules. The detected nodules is segmented. Then, a candidate set of features, such as volume, shape, and/or appearance features, are extracted and used for diagnosis. . . . . . . . . . . 16

7. The proposed framework for segmenting lungs from chest CT images. . . . . . 85

8. Test subject alignment and shape probability calculations. . . . . . . . . . 92

9. A typical 2D Illustration of how the shape probability for each voxel calculated in an adaptive way in terms of spatial locations based on varying window size and in terms of intensity value based on changing tolerance. . . . . . . . . . . 93

10. Axial planar sections of the 3D CT images; background / lung shape probabilities, and the final segmentation maps. . . . . . . . . . . . . . 95

11. Three different scales of the 3D Gaussian kernels (GK). . . . . . . . . . . 96 
12. 2D projection of the original and GSS filtered CT images on the axial (A), sagittal (S), and coronal (C) planes using 3D GKs shown in Figure 11. Original CT images(a); and the smoothed CT images (b,c,d) using the 3D GKs in Figure 11(a), Figure 11(b); and Figure 11(c), respectively. . . . . . . . . . . . 96

13. Axial planar sections of the original 3D CT image and its GSS-smoothed versions obtained with the $9 \times 9 \times 5,17 \times 17 \times 5$, and $33 \times 33 \times 5$ kernels. . . . 97

14. Typical 3D CT chest data $(a)$; and estimated density $(b)$ using only two dominant Gaussian components $(c)$, deviation between empirical and estimated densities $(d)$, estimated density of absolute deviation (e) LCDG components $(f)$, final estimated density $(g)$, and final estimated marginal density for each class $(h) .99$

15. 3D CT image (a) and its GSS-filtered version (b) for the $33 \times 33 \times 5$ kernel, with the final estimated conditional LCDG models for chest and lung marginals $(\mathrm{c}, \mathrm{d}) \ldots \ldots \ldots \ldots$. . . . . . . . . . . . . . . . . . . . . . . . . . . . . . . . . .

16. The 3D neighborhood system for pairwise MGRF model. . . . . . . . . . . . 100

17. Initial segmentation maps after voxel-wise classification by the LCDG modeling of original and GSS-smoothed images. . . . . . . . . . . . . 101

18. MGRF-guided segmentation of original image and its GSS smoothed version. . 103

19. Coronal 2D schematic illustration of the determination of segmentation measures for calculating the Dice similarity coefficient (DSC) . . . . . . . . . . 106

20. 3D schematic illustration for the absolute lung volume difference (ALVD) estimation. . . . . . . . . . . . . . . . . . 107

21. A schematic illustration for the Haussdorf distance (HD) calculation. . . . . . . 108

22. 3D lung segmentation (the UoLDB): 2D axial (first column), coronal (second column) and sagittal (third column) cross-sections of an original image (first row); its segmentation (second row); segmentation of the GSS-filtered images with $9 \times 9 \times 5$ (third row) and $17 \times 17 \times 5$ (forth row) kernels; and final segmentation (fifth row) with color-coded ground truth edges, false positive errors, and false negative errors (red, yellow, and pink points, respectively). Only these two kernels were used in the experiments, because after the second smoothing the overall segmentation accuracy improves insignificantly. . . . . . . . . . . . 109 
23. Impacts of elements of the proposed segmentation for 3 UoLDB subjects: original 2D chest sections (first row) and lung regions found for the original intensities and spatial interactions (second row); combined original/GSS-filtered intensities and spatial interactions (third row); and combined original/GSSfiltered intensities and spatial interactions, together with the adaptive shape model (fourth row). Same color ground truth and error coding as in Figure 22. 110

24. Other 4 subjects from the UoLDB: original CT chest images with different pathologies: (left: from top to bottom) lung mass; consolidation; lung cancer, and cavitation + consolidation pathologies, and the proposed segmentation (right: the same color ground truth and error coding as in Figure 22) . . . . . . . 111

25. Comparative segmentation of the UoLDB: First column- original CT sections with, from top to bottom, plural effusion and lung nodules; consolidation; pulmonary sarcoidosis, and fibrosis; second column- the proposed segmentation, and third column - the FC segmentation [1] (the same color ground truth and error coding as in Figure 22). . . . . . . . . . . . . . . 112

26. Comparative segmentation of the VESSEL [2] database: original CT sections (first row); The proposed segmentation (second row); and the FC segmentation (third row) [1] (the same color ground truth and error coding as in Figure 22). . 115

27. Lung separation process; (a) an original slice; (b) generated energies of pixels traversed until finding the optimum path, and (c) the original image with the overlaid separating path. . . . . . . . . . . . . . . 116

28. Segmenting pathological lungs from the LOLA11 database. First row-Original CT sections with, from left to right, fibrosis, lung mass (tumor), consolidation, cavitation + fibrosis, and fibrosis, and the proposed segmentation is in the second row (its accuracy has been evaluated by the LOLA11 organizers [3]). . . . . 120

29. Proposed segmentation result for 3 subjects from the LOLA11 database vs. the $2^{\text {nd }}$ - to $5^{\text {th }}$-rank LOLA11 competitors (the LOLA11 organizers [3] compared the extracted lungs with their ground truth and accounted for differences, being orange/pink-coded in the above region maps, to evaluate the overall accuracy). . 121

30. Total accuracy reduction due to erroneous segmentation of pathological tissues (first row) between ground truth (second row) and pathological-missing segmentation (third row): only $0.1 \%$ for missing the lung mass for a tumor (left column), but about $30 \%$ for a severe pathology (right column). . . . . . . . 123

31. Impacts of severe right and left lung pathologies on the proposed segmentation accuracy: the ground-truth-to-left-lung overlaps of $87.4 \%$ (left) and 5.2\% (right), the true lung maps being available to only the LOLA11 organizers. . . . 124 
32. The proposed framework for the co-alignment of lung CT images. . . . . . . . 128

33. Iso-surfaces generation: the reference and target distance maps $(a, b)$, and their iso-surfaces $(c, d) \ldots \ldots \ldots \ldots$. . . . . . . . . . . . . . . . . . . . . . . . . .

34. Schematic illustration of the $\mathrm{N}$-nearest voxels. . . . . . . . . . . . . . 131

35. GGMRF spatial refinement effect on the voxel-wise correspondences between two iso-surfaces. . . . . . . . . . . . . . . . . . . . . 134

36. 3D global and local registration results projected into axial, coronal, and sagittal planes: (a) reference objects with overlaid target edges (green); and the superimposed checkerboard visualization (b) before global alignment (SSD = 0.032), (c) after global alignment (SSD = 0.024), (d) after FFD alignment (SSD $=0.020)$, and $(\mathrm{d})$ after the iso-surfaces-alignment $(\mathrm{SSD}=0.015) \ldots \ldots 136$

37. 3D registration results: superimposed reference and target CT data before global alignment(a), after global alignment(c), after the iso-surfaces-alignment(e). and their respective superimposed checkerboard visualization(b), (d), and (f). Note that the reference and target objects are shown in grey and orange colors, respectively. . . . . . . . . . . . . . . . . . 137

38. Comparing the 1,309 randomly selected voxel positions from the lung vascular system for a large deformation field: The ground truth is in blue, and the corresponding positions found with the proposed method are in red. Note that all units are in $\mathrm{mm}$ in all directions. . . . . . . . . . . . . . . . . . . . . . . . 141

39. Effect of radiation on normal lung tissues; three axial slices from Pre-treatment scan (a), 3 months follow-up scan (b), and 6 months follow-up scan (c). Note that they are cropped from the same center coordinate to show the effect of global and local misalignment. . . . . . . . . . . . . . . . . 144

40. General framework for detecting the radiation-induced lung injuries (RILI). . . 147

41. Processing pipeline framework for detecting the radiation-induced lung injuries (RILI). . . . . . . . . . . . . . . . . . . . . 148

42. Successive 4D-CT data registration to obtain the final deformation field $\mathbf{u}(\mathbf{p})=$ $\sum_{i=1}^{N-1} \mathbf{u}_{i}(\mathbf{p})$ between the peak inhale and peak exhale phases. . . . . . . . . 149

43. Processing steps of the proposed framework for one subject: peak inhale (a); and voxel-wise $7^{\text {th }}$-order Gibbs energies (b) . . . . . . . . . . . . 153

44. The $7^{\text {th }}$-order clique (a): signals $q_{0}, q_{1}, \ldots, q_{6}$ are at the central pixel and its six central-symmetric neighbours at the radial distance $r$, respectively. . . . . . 155 
45. (a) baseline image $I_{b}$, (b) 3 months follow-up image $I_{f}$, (c) deformed image $I_{b}(T(x))$, and $(\mathrm{d})$ the difference image $I_{d}(\mathrm{~d}) \ldots \ldots \ldots \ldots \ldots \ldots$

46. Graphical illustration of the random forest (RF) classification. . . . . . . . 158

47. ROC curves for the RILI classification. . . . . . . . . . . . . . . . 162

48. Processing steps of the proposed framework for one subject: peak inhale (a) and peak exhale (b) images; the deformed inhale image (c); lung segmentation (d); voxel-wise $7^{\text {th }}$-order Gibbs energies (e); color-coded estimated Jacobian ventilation (f) and maximal strain component ( $\mathrm{g}$ ); and identified by classification injured region (h) with respect to the ground truth (false negative errors are pink-coded). . . . . . . . . . . . . . . . . . 163

49. More injured lung regions from different subjects (the regions were identified with the proposed framework and projected onto the axial plane; the ground truth was obtained by a radiologist). False positive and false negative errors are yellow- and pink-coded, respectively. . . . . . . . . . . . . . . . 164

50. More injured lung regions from different subjects identified using the proposed framework (coronal planar projections; the ground truth by a radiologist, false positive (yellow-coded) and negative (pink-coded) errors). . . . . . . . . . . 165 


\section{LIST OF ALGORITHMS}

$\begin{array}{ll}\text { ALGORITHM. } & \text { PAGE }\end{array}$

1. The Proposed Lung Segmentation Algorithm _ . . . . . . . . . . . . . 91

2. Adaptive shape prior ... . . . . . . . . . . . . . 94

3. Gaussian scale space smoothing . . . . . . . . . . . . . 97

4. $1^{\text {st }}$-order intensity modeling with linear combination of discrete Gaussians . 101

5. Learning the MGRF model of the input map. . . . . . . . . . . . 103

6. Solution of the Laplace Equation Between two Surfaces . . . . . . . . . . 132

7. Proposed Registration Methodology . . . . . . . . . . . . . . 135

8. 4D-CT segmentation using adaptive shape prior. . . . . . . . . . . 150

9. Learning the $7^{\text {th }}$-order MGRF appearance models. . . . . . . . . . . . 154 


\section{CHAPTER I INTRODUCTION}

Medical imaging is a very important emerging technology in the last few decades. It is used intensively in diagnosis, disease monitoring, assessment of treatment effectiveness, taking therapeutic or surgical decisions, and guiding surgery operations. Recent advances in medical imaging modalities provide images with a plethora of sizes, structures, resolution, and degrees of contrast. Such available data represents a very large amount of valuable information that is too huge to be exploited by radiologists and physicians. Therefore, the design of Computer Aided Diagnostic (CAD) system is of a great importance as an assistive tool for the medical community. A two-dimensional (2D) image is a rectangular array of pixels (picture cells, or elements) containing measured visual signals (intensities or colors) that quantify properties of related spatial locations in a whole body or its part. The $2 \mathrm{D}$ image of a thin planar cross-section of a 3D object is usually called a slice. A collection of the successive slices forms a 3D image, being an array of voxels (volume elements).

Each imaging modality has advantages and limitations in providing structural and/or functional physiological information about every organ of interest. Figure 1 exemplifies most important and popular modalities, namely, magnetic resonance imaging (MRI); computed tomography (CT); ultrasound imaging (US, or USI); positron emission tomography (PET), and single photon emission computed tomography (SPECT). The MRI and CT provide both the functional and structural information, whereas the USI, PET, and SPECT give the functional information. The structural MRI falls into three basic categories: the T1-, T2-, and PD (proton density)-weighted MRI. The functional MRI is classed into the fMRI; dynamic contrast enhanced MRI (DCE-MRI); tagged MRI; perfusion-weighted MRI (PWI), and diffusion MRI. The latter includes the diffusion-weighted imaging (DWI), 


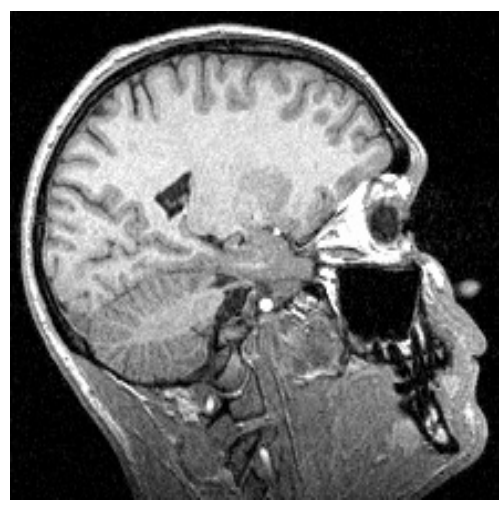

Brain MRI

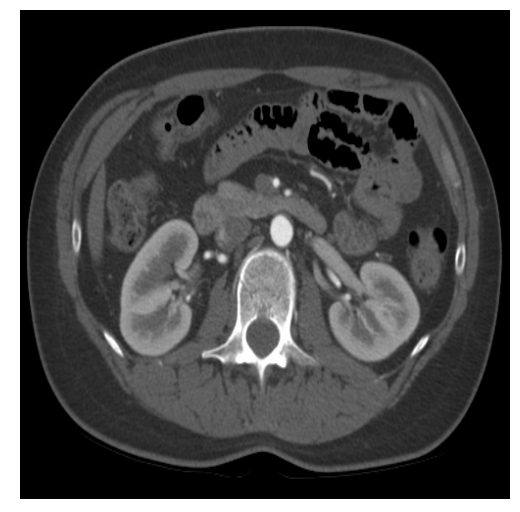

Kidney CT image

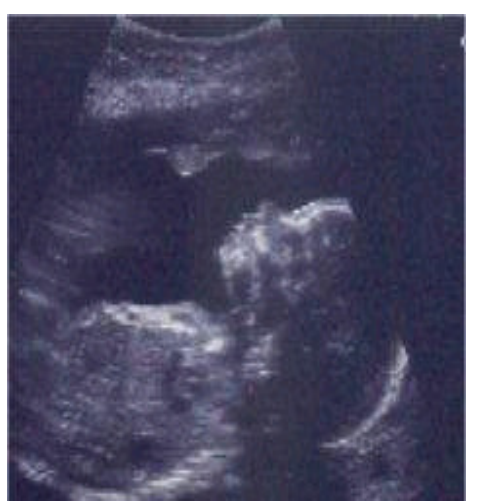

Fetus US image

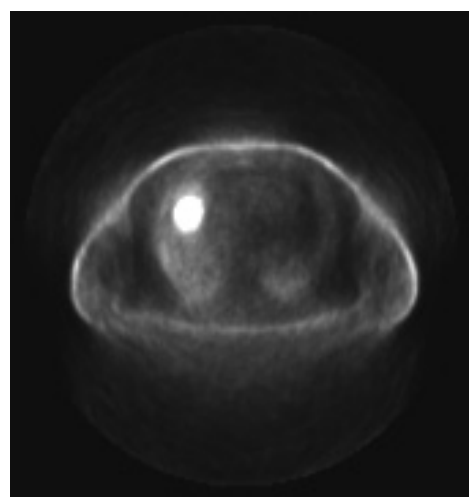

Lung PET image

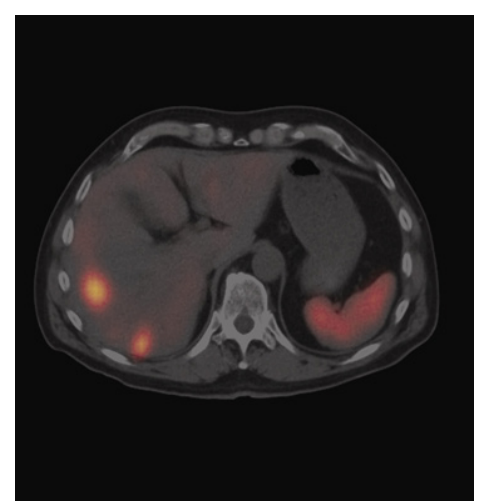

Liver SPECT image

FIGURE 1: Most popular types of medical images.

diffusion tensor imaging (DTI) and diffusion spectrum imaging (DSI). The functional CT is separated into the contrast enhanced CT (CE-CT) and the CT angiography (CTA).

The following section will focus only on CT and nuclear medical imaging as they are the related modality explored through the dissertation.

\section{A. Computed tomography (CT)}

One of the most popular and useful medical imaging modalities that has been available since the mid-1970, the CT reconstructs internal object structures from multiple projections obtained by measuring transmission of X-ray radiation through the object. The X-ray source and the set of detectors are mounted on the rotating gantry of the CT device, 
so that both the source and the detectors remain opposite to one another when revolving around, or scanning the patient's body. While passing through the patient's body, each individual X-ray beam is absorbed (attenuated) by muscles, fat, bones, and other tissues. The detector, being opposite to the X-ray source, measures the beam magnitude integrating all the attenuations due to absorption along the way from the source. The multiple projections, i.e., the integral absorptions by the patient's body for multiple directions of the beams, allow for reconstructing point-wise X-ray attenuation coefficients for each location within the object. The coefficients are measured in integer Hounsfield units (HU), called CT numbers, which vary from -1000 (air) to +1000 (cortical bone), 0 corresponding to water. The attenuation coefficient of the matter, measured in integer Hounsfield units (called CT numbers), is the intensity value of the volumetric CT scan. The HU values vary from $\mu_{\text {air }}-1000$ (air) to $\mu_{\text {bone }}=+1000$ (cortical bone), $\mu_{H_{2} O}=0$ corresponding to water, and a general $\mu$ $\mathrm{HU}$ corresponds to a material with attenuation coefficient $\frac{\mu-\mu_{\mathrm{H}_{2} \mathrm{O}}}{\mu_{\mathrm{H}_{2} \mathrm{O}}-\mu_{\text {air }}} \times 1000$. The CT scans $\mathrm{HU}$ are independent of scanner manufacturer and imaging sequence which make them as a direct quantitative measurement. The reconstructed CT images are 2/3D maps of these coefficients.

\section{Structural CT}

Historically, the CT images were acquired in an axial or transverse plane, being orthogonal to the long axis of the body. Recent developments in spiral and multi-slice CT enabled acquiring the volumetric (3D) images in a single patient breath-hold. For exploring anatomical structures, the volumetric data is easily represented by various planar cross-sections. Figure 2 shows typical axial 2D CT images.

Today's multi-detector CT scanners with 64 or more detectors obtain images with much finer details and in a shorter time. Continuous improvements in the scanning times and image resolution have dramatically increased the diagnostic accuracy. The structural CT allows radiologists to understand more in depth organs under examination and gain 

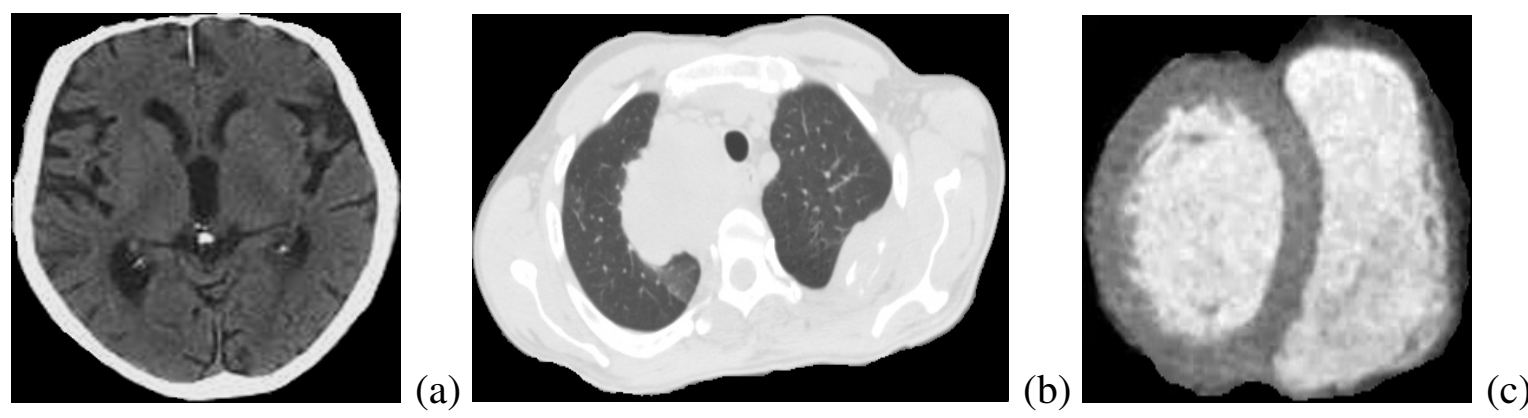

FIGURE 2: Axial brain (a), lung (b), and heart (c) CT scans.

valuable information for diagnosis and treatment.

\section{Contrast-enhanced CT (CE-CT)}

Although the CT readily acquires structural (anatomical) information, its abilities to provide functional (physiological) information are limited. Administering contrast agents before a CT scan helps in acquiring finer image details. The resulting CE-CT has benefits in both disease diagnosis and pre-operative guidance and planning, due to better contrast of anatomical structures, increased sensitivity of detecting pathologic lesions, and higher accuracy of lesion characterization. These benefits assist physicians in their clinical management.
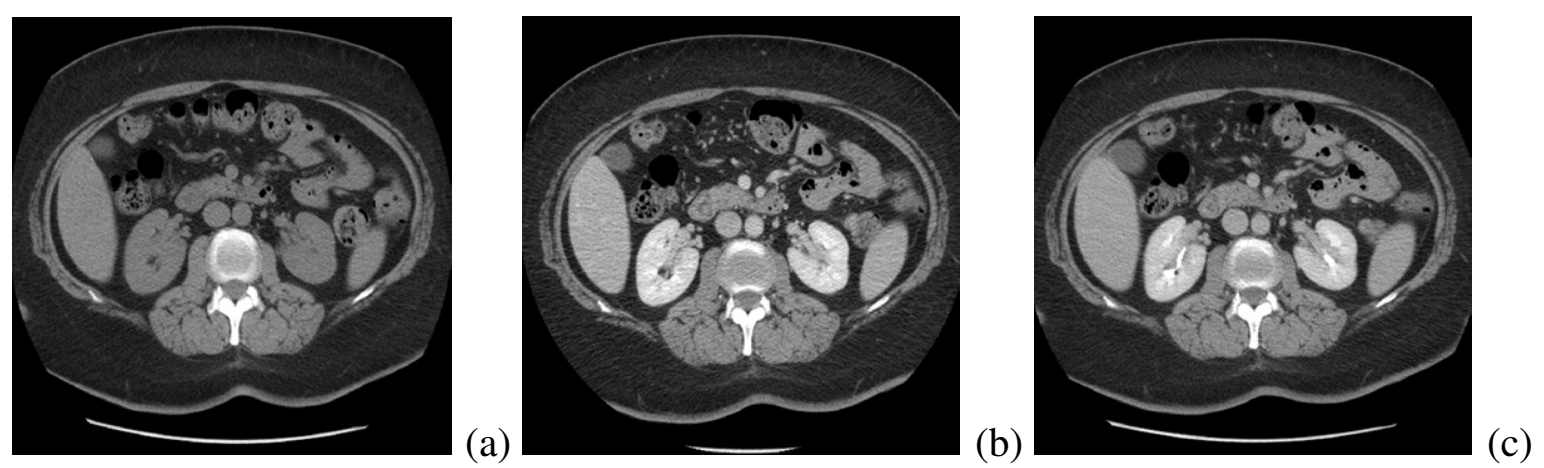

FIGURE 3: Abdominal DCE-CT scans before (a); $90 \mathrm{sec}$ after, and $180 \mathrm{sec}$ after administering the contrast agent.

Functional CT, called also dynamic $C E-C T$ (DCE-CT), takes a sequence of CT scans at the same location to study changes of the contrast agent distributions in time (fig- 
ure 3). The administration of contrast agents prior to the CT scan increases the image contrast and, therefore, helps to visualize finer details of tissues, organs, and physiological processes. Several types of non-iodinated (barium sulphate) and iodinated contrast agents are routinely used for both the traditional X-ray imaging (where these agents are often called radiocontrast ones) and the CT scans.

Generally, signal intensities in the X-ray imaging are proportional to electron densities. Since the early years of the X-ray radiography, a number of substances containing heavy elements with large atomic numbers, i.e., large numbers of electrons, were employed to achieve the better contrast. Depending on application, barium and iodine are the most common radiocontrast agents. The iodinated agents are either ionic (high-osmolar), or non-ionic (low-osmolar organic) compounds, the former having more side effects, than the latter.

The medical DCE-CT scanning generally uses an insoluble barium sulphate powder to block the X-rays passage, so that organs filled with it become white on an X-ray image. This universal contrast agent helps in the X-ray diagnosis of problems in the upper gastrointestinal areas, e.g., the oesophagus, stomach, and/or small intestine. Clear soluble iodine agents are generally harmless and usually injected as liquids into the body parts to be imaged. Most of the intravenous contrast dyes are iodine-based, so that the iodinecontaining agents are used for imaging the gallbladder, urinary tract, blood vessels, spleen, liver, bile duct and other soft tissues.

\section{CT angiography (CTA)}

CTA is a non-invasive examination that used to visualize blood flow in the vessels throughout the body, e.g. in arterial vessels through thr body, abdomen, lungs, kidneys, after an intravenous contrast agent was injected (figure 4).

The image recording during CTA examination takes only seconds, while most of the time is spent in setting up the requirements. In comparison with other imaging techniques, 


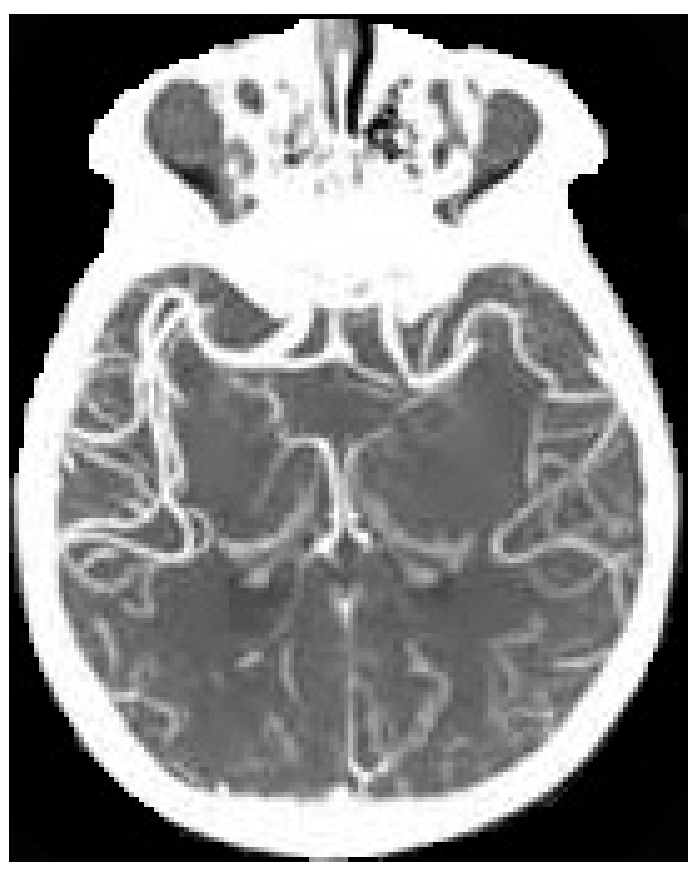

FIGURE 4: Brain CTA image.

it provides a lower cost at a faster scan time compared to other imaging modalities, and detecting aneurysms (enlarged blood vessels) and narrowing or damages of vessels early in time for determining appropriate therapies.

\section{Microtomography (Micro-CT)}

The micro-CT $(\mu \mathrm{CT})$ is a high-resolution CT for imaging small-scale internal structures. While spatial resolution of a typical medical CT scanner is about $1 \mathrm{~mm}$, the micro-CT systems can acquire 3D images with voxel spacing of less than $0.1 \mathrm{~mm}$ and perform 3D microscopy of internal structures by getting about 2,600 2D cross-sections in a single scan. These technical advances have made the micro-CT practical for both in vivo and in vitro studies that need high-resolution images, e.g. for biomedical research. 


\section{Dynamic CT imaging (4D-CT)}

For some clinic applications, such as radiation therapy planning, or identifying abnormal parathyroid, a time series of 3D lung CT scans is acquired during the breathing/respiratory cycle. These series are called 4D-CT or dynamic CT lung scans. It is used for the assessment of motion during breathing/respiratory cycle to improve the margins of the delivered radiation. The series of 3D-CT scans (4D-CT) are acquired during the free breathing with a scanner connected to respiration-monitoring system that used in the reconstruction of the temporal 3D volumes to a 4D-CT one.

\section{CT imaging: Pros and cons}

At present, the CT is faster and more widely available than other imaging modalities, results in low per-scan costs. Also, it is less likely to require sedating or anaesthetizing the patient, and it provides good spatial resolution with the ability to image bone, soft tissue and blood vessels all at the same time, compared with the MRI, i.e., the CT allows for separating two inner structures at smaller distances from each other. Also, it is less sensitive to patient movement and no radiation remains in the body after the examination.

The main concern of the $\mathrm{CT}$ is the use of ionizing X-ray radiation, which may be harmful to patients and can be accumulated by successive scans and cause DNA damage or induce cancer. Recent CT technologies use low radiation doses in order to reduce the radiation effects. However, the lower the dose, the lesser the image quality.

\section{B. Nuclear medical imaging (nuclide imaging)}

This modality visualizes spatial distribution of a radioactive decaying isotope (radionuclide), which has been administered into the body. Emitted gamma-rays or positrons are acquired by an appropriate radiation detector, e.g., a gamma-camera (figure 5). The resulting functional images are of low resolution, noisy, and without anatomic or structural 
details, but demonstrate metabolic or biochemical activities in the body, specific disease processes, or damaged areas. However, this imaging, like the CT, may be harmful to patients due to ionizing radiation.

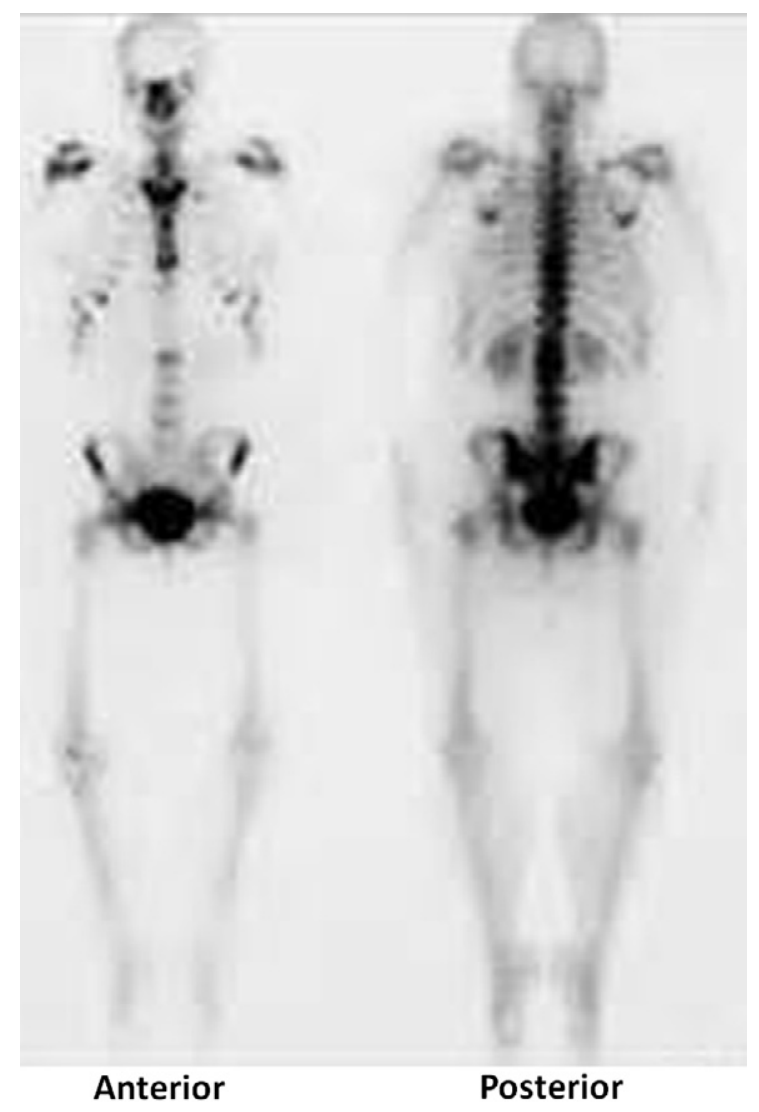

FIGURE 5: Nuclear medical imaging.

The emitted isotope radiation is quantified by measuring its half life (the time required for radioactivity to drop to half of its starting value), half-value layer, and energy. For imaging, the half life time should be short enough to reduce the patient radiation dose, but still sufficiently large to produce the image with radiation, which was not totally absorbed within the body. The half-value layer is the tissue thickness that absorbs half of the radioactivity produced. The layer of several centimeters indicates that most of the radiation is absorbed within the body, so that the required patient's radiation dose has to be too high. The radiation energy is usually measured in $\mathrm{KeV}$, the typical gamma-radiation energies in 
the nuclear medical imaging being between 100 and $200 \mathrm{KeV}$. Gamma-rays of the lower energies cannot leave the body to roduce images, while the higher energies cause image distortions due to scattered and unwanted radiation penetrating the gamma-camera. Table 1 shows the half lives and energies of radionuclides, being commonly used in the nuclear medical imaging. In particular, Thallium-20 emitting $X$-rays with energies between 69-82 $\mathrm{KeV}$ and photons of 135 and $167 \mathrm{KeV}$ is widely used for nuclear cardiac stress tests due to good imaging characteristics and reasonably small patient radiation doses. Iodine-123 and indium-111 isotopes have similar characteristics.

TABLE 1: Typical radionuclides for nuclear medical imaging.

\begin{tabular}{|c|c|c|c|c|}
\hline & & & \multicolumn{2}{|c|}{ Emission type: } \\
\cline { 4 - 5 } Radionuclide & Symbol & Half-life & Gamma $(\mathrm{KeV})$ & Positron $(\mathrm{KeV})$ \\
\hline Krypton-81m & ${ }^{81 \mathrm{~m}} \mathrm{Kr}$ & $13.1 \mathrm{sec}$ & 190 & - \\
Rubidium-82 & ${ }^{82} \mathrm{Rb}$ & $1.3 \mathrm{~min}$ & 511 & 3.4 \\
Nitrogen-13 & ${ }^{13} \mathrm{~N}$ & $10.0 \mathrm{~min}$ & 511 & 1190 \\
Fluorine-18 & ${ }^{18} \mathrm{~F}$ & $1.8 \mathrm{~h}$ & 511 & 250 \\
Technetium-99m & ${ }^{99 \mathrm{~m}} \mathrm{~T} \mathrm{c}$ & $6.0 \mathrm{~h}$ & 140 & - \\
Iodine-123 & ${ }^{123} \mathrm{I}$ & $13.0 \mathrm{~h}$ & 159 & - \\
Indium-111 & ${ }^{111} \mathrm{In}$ & 2.8 days & 171,245 & - \\
Thallium-201 & ${ }^{201} \mathrm{Tl}$ & 3.0 days & $69-82(X$-ray $)$ & - \\
Gallium-67 & ${ }^{67} \mathrm{Ga}$ & 3.2 days & $93,185,300,394$ & 0.36 \\
Xenon-133 & ${ }^{133} \mathrm{Xe}$ & 5.3 days & 81 & 0.81 \\
Iodine-131 & ${ }^{131} \mathrm{I}$ & 8.0 days & 364 & - \\
\hline
\end{tabular}

Typical nuclear medical images are produced by either a gamma-ray (rarely, $X$ ray) emission: a planar scintigraphy and single-photon emission computed tomography (SPECT), - or a positron emission tomography (PET), which can also be combined with the CT or MRI, e.g., the PET/CT, SPECT/CT, and SPECT/MRI. A positron is an elec- 
tron's antiparticle with opposite charge. Other types of radioactivity, such as, e.g., alpha-, beta-, or gamma-rays, are used only for therapeutic applications, where a radionuclide is targeting tumor locations in order to destroy diseased tissues. The gamma- and beta-rays are not used for imaging due to their too high energies and too small half-value layers. For positron emission, a radiopharmaceutical is synthesized in a cyclotron. Typical examples: carbon-11, oxygen-15, fluorine-18, and nitrogen-13, - are introduced into the body with biologically active molecules, in which one or more atoms have been replaced by their radioactive counterparts. The most common radiopharmaceutical in the clinical PET scanning is a sugar fluorodeoxyglucose, containing fluorine-18 and carbon-11 palmitate. A positron emitted by an radioisotope travels in the tissue for a short distance (typically, less than $1 \mathrm{~mm}$ depending on the isotope). Then it annihilates with an electron in the tissue, forming two gamma-rays that produce better image quality, than other radioactive decay mechanisms.

The planar scintigraphy visualizes a 2D distribution of a radioactive (radiopharmaceutical) material within the human body. Typically, the material is taken internally, and its emitted radiation is captured by a gamma camera. Clinically, these scintigraphic images are beneficial for obtaining information and making diagnostic conclusions about the size and normal/abnormal behavior of inner organs. Also, certain abnormalities, e.g., lesions or tumors, which are difficult to find with other imaging modalities, can be visually detected in these images as brighter or darker than normal areas of higher or lower concentration of radiation, respectively.

The planar scintigraphy is widely used for detecting bone fractures as areas of the increased radiation; thyroid metastases and functionality (using Iodine-131 or Technetium99m) or parathyroid adenomas; and a heart stress (usually, using Thallium-201) due to a coronary steal and ischemic coronary artery disease. It can also diagnose pulmonary embolism with a two-phase ventilation / perfusion scan; obstruction of the bile ducts by a gallstone or a tumor, and gallbladder diseases, e.g. bile leaks of biliary fistulas. In the 
brain imaging, the planar scintigraphy helps usually to detect tumors, indicated by higher radiation uptakes, since the blood flow is often higher in the tumor than other tissues, or confirm the brain death (dead brain functionally) if the visualized carotid arteries are cut off at the base of the skull.

The SPECT imaging exploits the same principles as the planar scintigraphy and a similar imaging procedure, but reconstructs, like the conventional CT, a 3D tomographic image from a set of 2D nuclear medical images taken from different views. A radiopharmaceutical is injected into the patient, e.g., through blood; multiple scintigraphic images are acquired from the different angles, and a 3D distribution of the radiation energy is reconstructed from these $2 \mathrm{D}$ images considered the tomographic projections.

The SPECT images are clinically beneficial for measuring blood perfusion in the brain to indicate strokes or the Alzheimer's disease, and myocardial perfusion and blood flow patterns in the heart to detect the coronary artery disease and myocardial infarct. Like the planar scintigraphy, these images can also be used for bone scanning and detecting tumors. Other applications include detecting liver diseases, e.g., cirrhosis; assessing the kidneysfunctionality, e.g., to reveal renal artery stenosis or renal infarction, and studying the lung perfusion to indicate bronchitis, asthma, or pulmonary edema.

The PET produces a 3D image that provides functional information about specific processes in the body. Contrary to the SPECT, the PET's gamma-camera detects pairs of gamma-rays, following the annihilation of an emitted positron. Detecting the pairs, rather than single gamma-rays, as in the planar scintigraphy and SPECT, allows the PET to produce images of better quality and resolution. However, the cost of the PET imaging is higher, and a cyclotron to provide the positron-emitting radionuclides, having typically very short half life times, must be available just on-site.

The PET has been used in many medical applications. In particular, the PET with the fluorodeoxyglucose radiopharmaceutical (FDG-PET) was widely used in exploring possibilities of cancer metastases to spread to other sites. Other positron-emitting radionu- 
clides are often used in the PET, too. For instance, the regional blood flow in the brain is measured with the radioactive oxygen-15, because its concentration is proportional to the brain perfusion and allows for detecting brain diseases associated with local areas of the increased or decreased uptake. In neurology, the PET is used in brain tumor evaluation and early identification of recurring degenerative disorders such as the Alzheimer's disease in order to plan assessment and surgical treatment of seizure disorders. The cardiac PET helps in measuring the blood flow and metabolism inside the heart; assessing myocardial fatty acid metabolism and infarction, and determining the need for a heart transplant or a bypass surgery.

The combined, or hybrid PET/CT, SPECT/CT, and PET/MRI add precise anatomic localizations and/or soft tissue images to functional nuclear imaging data. Both types of the images are captured sequentially, in the same session, and fused into a single superposed (co-registered) image. The PET/CT is typically used in lung cancer applications for diagnosing pulmonary nodules, surgical planning, radiation therapy, and cancer staging. The combined PET and CT images improve delineating lung nodule contours and estimating their volumes. The SPECT/CT helps in locating ectopic parathyroid adenomas, which may not be in their usual locations in the thyroid gland.

\section{Computed tomography Applications in different diseases}

Basics of the CT, including the attenuation corrections, CT numbers, windowing, back projection, and reconstruction are detailed in [4-6]. This imaging modality has been extensively used in clinical determination of a trauma's extent [7], tumor localization [8], detection of lung diseases [9-12], heart disease diagnosis [13], diagnosis of kidney diseases [14], study of dental problems [15] etc.

The DCE-CT has gained considerable attention for capturing parameters of physi-

ological functioning and disease in the human body and has many clinical applications in brain and neck tumor diagnosis [16], lung nodule evaluation [17], prostate cancer exami- 
nation [18], and therapy monitoring [19].

The CTA is widely used in clinical applications, in particular, for diagnozing the coronary artery disease of the heart [20], evaluating patients with acute ischemic stroke [21], diagnozing renal artery stenosis [22], and detecting acute pulmonary embolism [23].

Practical applications of the micro-CT, include investigation of small specimens and animals [24,25], evaluations of mineralized tissues, such as insect exoskeletons [26] and skeletal tissues [27], quantification of pulmonary fibrosis and investigation of airway microstructures of animals lungs [28], assessment of bone and soft tissue disease and therapeutic response of small animals [29], and assessment of induced cardiac stress [30].

\section{Dissertation organization}

The dissertation consists of six chapters. The following are summaries for the scope of each chapter:

- Chapter I presents some of the basic concepts about medical images and CT images. The chapter covers different types of CT images including structural CT, contrastenhanced CT (CE-CT), CT angiography (CTA), microtomography (Micro-CT), dynamic CT imaging (4D-CT), and discusses the advantages and disadvantages as well.

- Chapter II introduces a survey of the methodologies, challenges, and limitations of recent computer aided diagnosis (CAD) systems for lung cancer. It covers the four main components of designing any CAD system which are: $(i)$ segmentation of lung tissues, (ii) detection of the nodules which are inside the lung parenchyma, (iii) segmentation of detected lung nodules, and (iv) classifications of the nodules as benign or malignant. It goes through each component and explores all related recent work. Also, the limitations of the existing techniques have been reported and how to overcome these limitations.

- Chapter III introduces the first and most important step in developing any CAD sys- 
tem for lung cancer, which is the lung segmentation. It introduces an accurate lung parenchyma segmentation methodology with the focus of pathological lungs to extract the volume of interest (VOI) to be analyzed for potential existence of injured lung tissues.

- Chapter IV introduces the second crucial step in developing any CAD system for lung cancer, which is the lung registration. It ensures voxel on voxel matching between different 3D lung volumes by deforming each voxel of the source object over a set of nested, equi-spaced surfaces (i.e., iso-surfaces) to closely match the target object. The developed non-rigid registration exploits the geometric features in addition to image intensities to avoid problems associated with nonlinear intensity variations in medical images.

- Chapter V presents the general framework of the lung injury detection through the estimation of features for lung tissues and its classification as normal or injured.

- Chapter VI concludes the work and outlines the future directions. 


\section{CHAPTER II}

\section{COMPUTER-AIDED DIAGNOSIS SYSTEMS FOR LUNG CANCER: A SURVEY}

This chapter overviews one of the most important, interesting, and challenging problems in oncology, which is the problem of lung cancer diagnosis. Developing an efficient computer-aided diagnosis (CAD) system for lung cancer is of great clinical importance and can increase the patient's chance of survival. For this reason, developing CAD systems for lung cancer has been investigated in a huge number of research studies. A typical CAD system for lung cancer diagnosis is composed of four main processing steps: segmentation of the lung fields, detection of the nodules inside the lungs, segmentation of the detected nodule, and diagnosing the nodules as benign or malignant. This chapter overviews the state-of-the-art techniques that are developed to implement each of these CAD processing steps. For each technique involved in this chapter, various aspects of technical issues, implemented methodologies, training and testing data, validation methods, as well as the achieved performance are described. In addition, the chapter addresses the several challenges that face the researchers in each implementation step and outlines the strengths and drawbacks of the exciting approaches for lung cancer CAD systems.

\section{A. Introduction}

Lung cancer remains the leading cause of cancer-related deaths in the US. In 2014, there were approximately 224,210 new cases of lung cancer, 116,000 Male and 108,210 Female, and 159,260 related deaths, 86,930 Male and 72,330 Female, [31]. Early diagnosis can improve the effectiveness of treatment and increase the patient's chance of survival. computed tomography (CT), low dose computed tomography (LDCT), contrast- 
enhanced computed tomography (CE-CT) and Positron emission tomography (PET) are most common non-invasive imaging modalities for detecting lung nodules and diagnosing the detected ones. PET scans are used to discriminate between malignant and benign lung nodules. Early detection of the nodules can be based on CT and LDCT scans that allow for reconstructing the anatomy of and detecting any anatomic change in the chest. The CE-CT allows for reconstructing the anatomy of the chest and assessing the diagnostics of the detected nodules.

A wealth of known publications have investigated the development of CAD systems for lung cancer from a host of different image modalities. The success of a particular CAD system can be measured in terms of accuracy, processing time, and automation level. The goal of this chapter is to overview the different CAD systems for lung cancer, proposed in the literature.

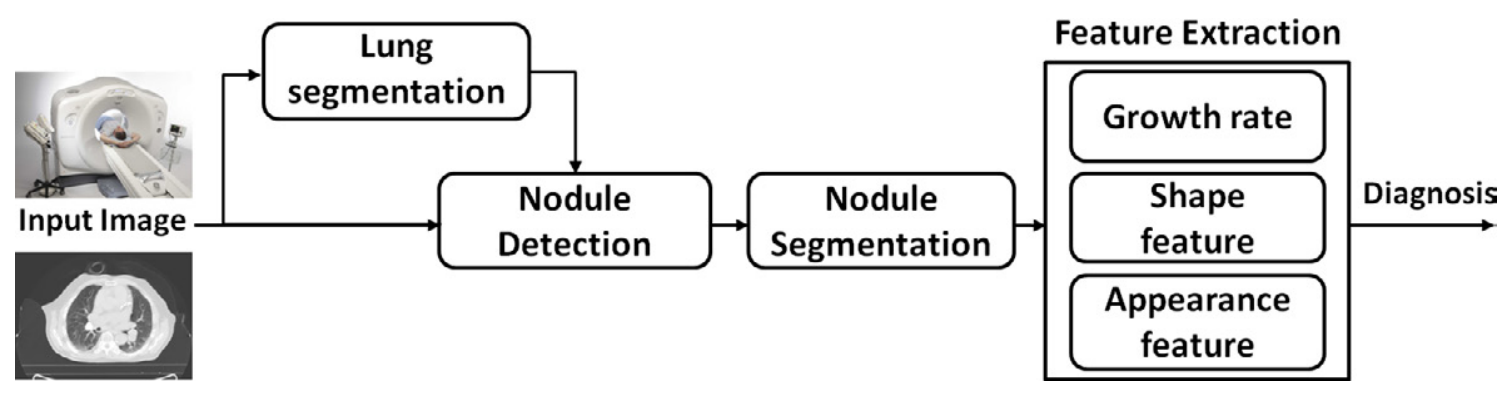

FIGURE 6: A typical computer-aided diagnosis for lung cancer. The input of the CAD system is the medical images obtained using the appropriate modality. A lung segmentation step is used to reduce the search space for lung nodules. Nodule detection is used to identify the lung nodules. The detected nodules is segmented. Then, a candidate set of features, such as volume, shape, and/or appearance features, are extracted and used for diagnosis.

A schematic diagram of a typical CAD system is shown in Figure 6. The segmentation of lung tissues on chest images is a pre-processing step in developing the CAD system in order to reduce the search space for lung nodules. Next, the detection and segmentation of the lung nodules from the available search space are mandatory steps. Finally, the clas- 
sification of the detected nodules into benign and malignant is the final step. Classification of the detected nodules is a major component in CAD schemes for detection and diagnosis of lung nodules in CT. In CAD schemes for detection (often abbreviated as CADe), a classification component classifies the nodule candidates identified in the previous step into nodules or non-nodules (i.e., normal anatomic structures), whereas a CAD scheme for diagnosis (often abbreviated as CADx) classifies detected nodules (either by a computer or a radiologist) into benign or malignant nodules. Below, we will address each processing step in developing CAD systems, i.e, the lung segmentation, the nodule detection, the nodule segmentation, and nodule diagnostics.

\section{B. Lung Segmentation}

The segmentation of lungs from chest images is a crucial step in any computer aided diagnosis system that can lead to the early diagnosis of lung cancer, as well as other pulmonary diseases. The segmentation of lungs is a very challenging problem due to the natural signal inhomogeneities over the lung region, very close signals of pulmonary structures such as arteries, veins, bronchi and bronchioles, and the different scanners and scanning protocols. A wealth of known publications has addressed the segmentation of lung regions from CT images and chest radiographs. The success of a particular technique can be measured in terms of accuracy, processing time, and automation level. Most of the existing techniques for healthy $\backslash$ moderate pathology lung segmentation can be classified into four categories based on signal thresholding, deformable boundaries, shape models, or edges.

Healthy lung tissues form darker regions in CT images compared to other parts of the chest such as heart and liver. This fact has encouraged many researchers to search for an optimum threshold that separates the lungs from all other tissues. Hu et al. [32] compute iteratively such a threshold to get an initial lung region. Then the initial segmentation is refined by opening and closing morphological operations. This method was further used by Ukil et al. [33] and van Rikxoort [34] to automatically segment the lung 
as a pre-processing step for the lung lobe segmentation. Ross et al. [35] used a similar method to the Hu et al. approach [32], but used Otsu's method [36] for thresholding, instead of the iterative thresholding, in order to segment the lung fields as a step of lung lobe extraction. Yim et al. [37] extract the lung fields by region growing followed by connected component analysis. Armato III et al. [38,39] used gray-level thresholds to segment first the thorax from the background, and then the lungs from the thorax. A rolling ball filter was further applied to the segmented lung borders to avoid the loss of juxtapleural nodules. The identified lung fields were used to limit the search space for their lung nodule detection framework. In [40], the threshold is selected automatically as described by Armato III et al. [38]. A thresold-based region filling methodology is then used to segment the lung fields as a first step for a pulmonary fissure segmentation framework. Pu et al. [41] set a threshold to initially segment the lung regions. To refine the segmentation and include juxtapleural nodules, a border marching algorithm is used to march along the the lung borders with an adaptive marching step and refine convex tracks. Gao et al. [42] proposed another threshold-based segmentation approach consisting of four processing steps: $(i)$ removing the large airway from the lung region by an isotropic diffusion to smooth edges followed by region growing; (ii) finding an optimal threshold to remove pulmonary vessels; (iii) separating the left and the right lungs by detection of anterior and posterior junctions using the largest threshold, and (iv) morphological smoothing of the lung boundary along the mediastinum and lung wall based on the structure of the airway tree. To identify lung fields in a lung lobe segmentation framework, Wei et al. [43] selected a threshold to segment the lung regions using histogram analysis. The segmented lungs are then refined using connect-component labeling and circular morphology closing. Ye et al. [44] used a 3D adaptive fuzzy thresholding to segment the lung region from CT data. The segmentation is followed by smoothing the segmented lung contour, represented as chain code [45], by 1D Guassian smoothing. They further applied a methodology to detect the lung nodules in the segmented lung fields. The main problem of the threshold-based segmentation is that its 
accuracy is affected by many factors, including an image acquisition protocol and a scanner type (e.g., GE, Siemens, etc.). Moreover, radiodensities (in the Hounsfield units) of some pulmonary system's structures, such as arteries, veins, bronchi, and bronchioles, are very close to radiodensity of the chest tissues. As a result, the threshold-based segmentation cannot be accurate for the whole lung region and needs further intensive post-processing steps to overcome the natural signal inhomogeneity over the lung region.

Lung segmentation techniques of the second category use deformable boundary models, such as active contours (snakes), level sets, or geodesic active contours. A snake starts from some initial position and shape and evolves under specific internal and external guiding forces to fit the shape of one or more desired objects. Snakes can extract a region of interest (ROI) or locate an object boundary. Itai et al. [46] extract the lung region with a 2D parametric deformable model using the lung borders as an external force. The deformable model started from an initial segmentation obtained by a threshold estimated from CT data. The segmentation results were used as a pre-processing step to classify abnormal areas within each lung filed. Silveira et al. [47] used a 2D geometric active contour (level set) being initialized at the boundary of the chest region. Then it is automatically split into two regions representing the left and right lungs. Main drawbacks of the deformable model-based segmentation are the excessive sensitivity to initialization and the inability of traditional external forces (e.g., based on edges, gray levels, etc.) to capture natural inhomogeneity in the lung regions. As a result, it is hard to provide an adequate guidance to the deformable model to achieve the accurate segmentation.

To improve the segmentation accuracy, the shape-based techniques add prior information about the lung shape to image signals. To use the shape prior, it should be aligned with the initial CT data before starting the segmentation. Annangi et al. [48] integrated a prior shape term, calculated as described in [49], with a term describing edge feature points, and term representing region-based data statistics [50] in a variational energy framework for lung segmentation. The formulated energy is used to guide a level-set deformable model 
in order to segment the lung fields from posterior-anterior (PA) chest x-ray images. Shi et al. [51] used an adaptive shape prior to guide a deformable model used to segment the lung fields from time-series data. The initial shape is trained from manually marked lung field contours from the population using the principle component analysis (PCA) method and is used to segment the initial time-point images of each subject. To address the shape variability for each subject, the shape is adapted for the segmentation of further time-point images with the previously segmented images from the same subject. Van Ginneken et al. [52] optimized the active shape model (ASM) developed by Tsai et al. [53] to segment the lung fields. They compared the segmentation with an active appearance model (AAM)based segmentation and multi-scale resolution pixel classification, concluding that the latter gives the best results. Hardie et al. [54] invoked the optimized ASM shape model of van Ginneken et al. [52] to segment the lungs field in a CAD system developed to identify lung nodules on CT images. Sun et al. [55] segmented the lungs in two main processing steps. First, a 3D ASM matching method is used to get a rough initial segmentation of the lung borders. Second, a global optimal surface finding method, developed by Li et al. [56], is used to find a refined smoothed segmentation of the lungs.Sluimer et al. $[57,58]$ proposed to segment a pathological lung by using the shape model of a normal lung. Sofka et al. [59] aligned a shape model using a set of automatically detected anatomical landmarks and refined the shape model through an iterative surface deformation approach in order to segment lungs that involve pathologies. The main limitation of the shape-based segmentation techniques is that their accuracy depends strongly on how accurately the prior shape model is registered with respect to the $\mathrm{CT}$ image. Instead of using a shape prior, Kockelkorn et al. [60] used a user-interactive framework for lung segmentation in CT scans with severe abnormalities, where a user corrects the results obtained by a $k$-nearest-neighbor $(\mathrm{KNN})$ classifier trained on prior data.

Besbes et al. [61] used a graph-based shape model with image cues based on boosted features to segment the lung fields from chest radiographs. Hua et al. [62] presents an au- 
tomatic method to segment pathological lung fields using graph-based search of a cost function that incorporates the intensity, gradient, boundary smoothness, and the rib information. El-Baz et al. $[63,64]$ proposed an iterative Markov random field (MRF)-based segmentation framework to segment the lung fields from LDCT images. A linear combination of discrete Gaussian (LCDG) model with positive and negative components is used to approximate the empirical distribution of the LDCT signals of the lung fields and their background, describing the 1st-order visual appearance model of the LCDG image. An initial segmentation of the lung fields is obtained by voxel-wise Bayesian maximum A posteriori (MAP) classification of a given image, based on its LCDG approximation of the signals of the lung fields and their background. The segmentation of the lung fields is iteratively refined by the iterative conditional mode (ICM) relaxation that maximizes a MRF energy that account for the 1st-order visual appearance model and the spatial interaction between the image voxels.

The edge-model-based lung segmentation is performed using spatial edge-detector filters, or wavelet transforms. Campadelli et al. [65] detect an inial outline of lung borders by using the first derivative of Gaussian filters taken at four different orientations. Then, an edge tracking procedure using the Laplacian of Gaussian (LoG) operator at three different scales is used to find a continuous external lung contour, which is further integrated with the initial outline to produce the final lung segmentation from PA chest radiographs. Mendonça et al. [66] selected automatically the regions-of-interest from PA chest radiographs as rectangular areas that surrounds as close as possible each lung field through an iterative procedure. Edge points (i.e., the mediastinal, costal, top, and bottom edge points) are detected using spatial edge-detector filters and combined to define a closed contour for the lung borders.

A review of the current methodologies for lung fields segmentation is presented in Table 2. To efficiently reduce the search space for lung nodules, some technical issues should have further investigated to provide accurate segmentation of the lung fields. 
These technical issues include the automation level of the technique, the sensitivity of the method to the scanning parameters, the efficiency of an algorithm to work with different image modalities (e.g. CT, LDCT, or CE-CT), and the ability of the algorithm to provide a proper lung segmentation in cases with severe pathologies that are associated with inhomogeneities in the pathological lungs.

TABLE 2: Review of current lung segmentation approaches ordered by their publication year.

\begin{tabular}{|c|c|c|c|c|}
\hline Study & $\begin{array}{c}\text { Data Description } \\
\text { \& AL }\end{array}$ & oach & Speed & $\begin{array}{c}\text { Validation } \\
\text { and Accuracy }\end{array}$ \\
\hline $\begin{array}{ll}\text { Hu } & \text { et } \\
\text { al. [32] } & \end{array}$ & $\begin{array}{l}\text { - } 24 \quad \text { datasets } \\
\text { from } 8 \text { subjects } \\
\text { - } 512 \text { x } 512,3 \mathrm{~mm} \\
\text { thin } \\
\text { - A }\end{array}$ & $\begin{array}{l}\text { - } 3 \mathrm{D} \\
\text { - } \\
\text { Iterative thresh- } \\
\text { old } \\
\text { - } \text { dynamic pro- } \\
\text { gramming } \\
\text { - } \text { morphological } \\
\text { operations }\end{array}$ & $\begin{array}{l}2-3 \mathrm{~min} \text { on a } 300 \\
\mathrm{MHz} \text { processor, } \\
512 \mathrm{MB} \text { RAM } \\
(512 \times 512 \times 120)\end{array}$ & $\begin{array}{l}\text { - } 229 \text { Manual traced } \\
\text { images } \\
\text { - } \mathrm{RMSD}=0.54 \mathrm{~mm} \\
(0.8 \text { pixel })\end{array}$ \\
\hline $\begin{array}{l}\text { Mendonça } \\
\text { et al. [66] }\end{array}$ & $\begin{array}{l}\text { - } 47 \text { image radio- } \\
\text { graphs } \\
\text { - } \mathrm{A}\end{array}$ & $\begin{array}{ll}- & 2 \mathrm{D} \\
- & \text { Spatial edge de- } \\
& \text { tector }\end{array}$ & - & $\begin{array}{l}\text { - } 47 \text { Manual traced } \\
\text { data } \\
\text { - } \mathrm{SEN}=0.9225, \mathrm{PPV}= \\
0.968\end{array}$ \\
\hline $\begin{array}{l}\text { Yim et } \\
\text { al. [37] }\end{array}$ & $\begin{array}{l}\text { - } 10 \text { subjects } \\
\text { - } 512 \text { x 512, } \\
0.75-2 \mathrm{~mm} \text { thin } \\
\text { - } \mathrm{A}\end{array}$ & $\begin{array}{ll}- & 3 \mathrm{D} \\
- & \text { Region growing } \\
\text { - } & \text { Connected com- } \\
& \text { ponent }\end{array}$ & $\begin{array}{l}42.3 \mathrm{sec} \text { on } \mathrm{a} \\
2.5 \mathrm{GHz} \text { proces- } \\
\text { sor, } 2.0 \mathrm{~GB} \text { RAM } \\
(512 \times 512 \times 352)\end{array}$ & $\begin{array}{l}\text { - } 10 \text { Manual traced } \\
\text { data } \\
\text { - } \text { RMSD = 1.2pixel }\end{array}$ \\
\hline $\begin{array}{l}\text { Sluimer et } \\
\text { al. }[58]\end{array}$ & $\begin{array}{l}\text { - } 26 \text { scans } \\
\text { - } 512 \quad \text { x } 512, \\
0.75-2.0 \mathrm{~mm} \\
\text { - } \mathrm{A}\end{array}$ & $\begin{array}{l}\text { - } 3 \mathrm{D} \\
\text { - Shape-based }\end{array}$ & $\begin{array}{l}3 \mathrm{hr} \text { on a } 2.8 \mathrm{GHz} \\
\text { processor, } 2.0 \\
\text { GB RAM }(512 \times \\
512 \times 400)\end{array}$ & $\begin{array}{l}\text { - } 10 \text { Manual trace data, } \\
\text { each } 4 \text { slice } \\
\text { - } \mathrm{OM}=0.8165, \mathrm{AD} \\
=1.48 \mathrm{~mm}, \mathrm{HD}= \\
13.45 \mathrm{~mm}\end{array}$ \\
\hline $\begin{array}{l}\text { Campadelli } \\
\text { et al. }[65]\end{array}$ & $\begin{array}{l}\text { - } 487 \text { image ra- } \\
\text { diographs } \\
\text { - } 256 \times 256 \\
\text { - A }\end{array}$ & $\begin{array}{ll}- & 2 \mathrm{D} \\
- & \text { Spatial edge de- } \\
& \text { tector }\end{array}$ & - & $\begin{array}{l}\text { - } 487 \text { Manual traced } \\
\text { data } \\
\text { - } \mathrm{SEN}=0.9174, \mathrm{SPE} \\
=0.9584, \mathrm{PPV}= \\
0.9197, \quad \mathrm{ACC}= \\
0.9437\end{array}$ \\
\hline $\begin{array}{l}\text { Korfiatis } \\
\text { et al. [67] }\end{array}$ & $\begin{array}{l}\text { - } 23 \text { scans } \\
\text { - } 512 \text { x } 512, \\
0.625 \mathrm{~mm} \\
\text { - } \mathrm{A}\end{array}$ & $\begin{array}{ll}\text { - } & 3 \mathrm{D} \\
\text { - } & \text { Wavelet edge de- } \\
& \text { tector }\end{array}$ & $\begin{array}{l}3 \mathrm{~min} \text { on a } 2.8 \\
\mathrm{GHz} \text { processor, } 2 \\
\mathrm{~GB} \text { RAM }(512 \times \\
512 \times 50)\end{array}$ & $\begin{array}{l}\text { - } 22 \text { Manual traced } \\
\text { data } \\
\text { - } \mathrm{OM}=0.983, \mathrm{AD}= \\
0.77 \mathrm{~mm}, \mathrm{RMSD}= \\
0.52 \mathrm{~mm}\end{array}$ \\
\hline $\begin{array}{ll}\text { Gao } & \text { et } \\
\text { al. [42] } & \end{array}$ & $\begin{array}{l}\text { - } 8 \text { subjects } \\
\text { - } 512 \text { x } 512 \text { x } 240 \\
\text { - } \mathrm{A}\end{array}$ & $\begin{array}{l}\text { - } 2 \mathrm{D} \\
\text { - Thresholding }\end{array}$ & $\begin{array}{l}15-20 \mathrm{~min} \text { on a } \\
3.0 \mathrm{GHz} \text { proces- } \\
\text { sor, } 1 \mathrm{~GB} \text { RAM } \\
(512 \times 512 \times 240)\end{array}$ & $\begin{array}{l}\text { - } 8 \text { Manual traced } \\
\text { datasets } \\
\text { - } \text { DSC = 0.9946 }\end{array}$ \\
\hline
\end{tabular}


TABLE 2 - continued from the previous page

\begin{tabular}{|c|c|c|c|c|}
\hline Study & $\begin{array}{c}\text { Data Description } \\
\text { \& AL }\end{array}$ & oach & Speed & $\begin{array}{c}\text { Validation } \\
\text { and Accuracy }\end{array}$ \\
\hline $\begin{array}{l}\text { Silveira et } \\
\text { al. [47] }\end{array}$ & $\begin{array}{l}\text { - } 1 \text { subject } \\
\text { - } 512 \text { x 512, 1mm } \\
\text { thin } \\
\text { - A }\end{array}$ & $\begin{array}{ll}\bullet & 2 \mathrm{D} \\
- & \text { Deformable } \\
& \text { model }\end{array}$ & - & $\begin{array}{l}\text { - } \text { NA } \\
\text { - Qualitative assess- } \\
\text { ment }\end{array}$ \\
\hline $\begin{array}{l}\mathrm{Pu} \text { et } \\
\text { al. [41] }\end{array}$ & $\begin{array}{l}\text { - } 20 \text { datasets } \\
\text { - } 512 \times 512,1.25 \\
\text { mm thin } \\
\text { - } \mathrm{A}\end{array}$ & $\begin{array}{ll} & \text { 2D } \\
- & \text { Thresholding }\end{array}$ & $\begin{array}{l}1 \mathrm{~min} \text { on a } 2.11 \\
\mathrm{GHz} \text { processor, } 2 \\
\text { GB RAM }(512 \times \\
512 \times 540)\end{array}$ & $\begin{array}{l}\text { - } 20 \text { manual traced } \\
\text { datasets } \\
\text { - } \mathrm{FP} / \mathrm{GT}=0.43 \% \\
\mathrm{FN} / \mathrm{GT}=1.63 \%\end{array}$ \\
\hline $\begin{array}{l}\text { Shi et } \\
\text { al. [51] }\end{array}$ & $\begin{array}{l}\text { - } 247 \text { image ra- } \\
\text { diographs } \\
\text { - } 256 \times 256 \\
\text { - A }\end{array}$ & $\begin{array}{ll}- & \text { 2D } \\
- & \text { Shape-based de- } \\
& \text { formable model }\end{array}$ & $\begin{array}{l}75 \text { sec per im- } \\
\text { age on a } 3 \mathrm{GHz} \\
\text { processor, } 1 \mathrm{~GB} \\
\text { RAM }(512 \times 512)\end{array}$ & $\begin{array}{l}\text { - } 247 \text { Manual traced } \\
\text { images } \\
\text { - } \mathrm{OM}=0.92 \mathrm{AD}= \\
\text { 1.78pixel }\end{array}$ \\
\hline $\begin{array}{l}\text { El-Baz et } \\
\text { al. }[63,64]\end{array}$ & 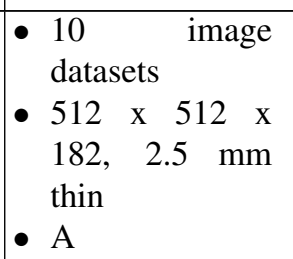 & $\begin{array}{ll}\text { - } & \text { 3D } \\
\text { - } & \text { Statistical MRF } \\
& \text { model }\end{array}$ & $\begin{array}{l}1.65 \text { sec per im- } \\
\text { age on a } 3.2 \mathrm{GHz} \\
\text { processor, } 16 \mathrm{~GB} \\
\text { RAM }\end{array}$ & $\begin{array}{l}\text { - } 1820 \text { Manual traced } \\
\text { images } \\
\text { - } \mathrm{ACC}=0.968\end{array}$ \\
\hline $\begin{array}{l}\text { Annangi } \\
\text { et al. [48] }\end{array}$ & $\begin{array}{l}\text { - } 1130 \text { image ra- } \\
\text { diographs } \\
\text { - } 128 \times 128 \text { \& } \\
256 \times 256 \\
\text { - A }\end{array}$ & $\begin{array}{ll}- & \text { 2D } \\
- & \text { Shape-based de- } \\
& \text { formable model }\end{array}$ & $\begin{array}{l}7 \text { sec per image } \\
\text { on a } 2.4 \mathrm{GHz} \text { pro- } \\
\text { cessor }\end{array}$ & $\begin{array}{l}\text { - } \text { Manual traced data } \\
\text { - } \mathrm{DSC}=0.88\end{array}$ \\
\hline $\begin{array}{l}\text { Kockelkorn } \\
\text { et al. }[60]\end{array}$ & $\begin{array}{ll}\text { - } 22 \text { scans } & \\
- & 0.9-1.0 \quad \mathrm{~mm} \\
& \text { thickness } \\
\text { - } & \mathrm{UI}\end{array}$ & $\begin{array}{ll}- & 3 \mathrm{D} \\
\text { - } & \text { Prior training } \\
\text { - } & \text { statistical classi- } \\
& \text { fier }\end{array}$ & $10 \mathrm{~min}$ & $\begin{array}{l}12 \text { Manual traced } \\
\text { data } \\
\text { - } \mathrm{OM}=0.96, \mathrm{AD}= \\
1.68 \mathrm{~mm}\end{array}$ \\
\hline $\begin{array}{l}\text { Besbes et } \\
\text { al. [61] }\end{array}$ & $\begin{array}{l}\text { - } 247 \text { image ra- } \\
\text { diographs } \\
\text { - } 256 \times 256,1 \mathrm{~mm} \\
\text { thin } \\
\text { - A }\end{array}$ & $\begin{array}{l}\text { - 2D } \\
\text { - Shape-based }\end{array}$ & - & $\begin{array}{l}\text { - } 123 \text { Manual traced } \\
\text { data } \\
\text { - } \mathrm{OM}=0.9474, \mathrm{AD}= \\
\text { 1.39pixel }\end{array}$ \\
\hline $\begin{array}{l}\text { Sofka et } \\
\text { al. [59] }\end{array}$ & $\begin{array}{ll}\text { - } & 260 \text { scans } \\
\text { - } & 0.5-5.0 \quad \mathrm{~mm} \\
& \text { thickness } \\
\text { - } & \\
& \end{array}$ & $\begin{array}{l}\text { - } 3 \mathrm{D} \\
\text { - Shape-based }\end{array}$ & - & $\begin{array}{l}\text { - } 68 \text { Manual traced } \\
\text { data } \\
\text { - } \mathrm{SCD}=1.95\end{array}$ \\
\hline $\begin{array}{l}\text { Hua et } \\
\text { al. [62] }\end{array}$ & 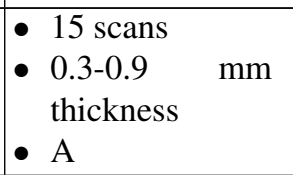 & $\begin{array}{l}\text { - 3D } \\
\text { - Graph-search }\end{array}$ & $\begin{array}{l}6 \mathrm{~min} \text { on a } 2.0 \\
\mathrm{GHz} \text { processor, } \\
32 \mathrm{~GB} \text { RAM }\end{array}$ & $\begin{array}{l}\text { - } 12 \text { Semi-automated } \\
\text { traced data } \\
\text { - } \mathrm{HD}=13.3 \text { pixel, SEN } \\
=0.986, \mathrm{SPE}=0.995\end{array}$ \\
\hline $\begin{array}{ll}\text { Sun et } \\
\text { al. [55] }\end{array}$ & $\begin{array}{ll}\text { - } 30 \text { scans } \\
\text { - } 512 \quad \text { x512 } \\
\mathrm{x} \quad 424-642, \\
0.6-0.7 \quad \mathrm{~mm} \\
\text { thin } \\
\text { - } \mathrm{A} \\
\end{array}$ & $\begin{array}{l}\text { - } 3 \mathrm{D} \\
\text { - Shape-based }\end{array}$ & $\begin{array}{lr}6 \text { min per dataset } \\
\text { on a NVIDIA } \\
\text { Tesla } & \text { C1060 } \\
\text { processor } & (240 \\
\text { thread), } 4 & \text { GB } \\
\text { RAM } & \\
\end{array}$ & 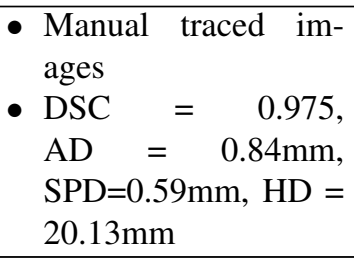 \\
\hline DSC: & \multicolumn{4}{|c|}{$\begin{array}{l}\text { denotes the Dice similarity coefficient:DSC }=\frac{2 * \mathrm{TP}}{2 * \mathrm{TP}+\mathrm{FP}+\mathrm{FN}}, \\
\text { where TP: true positive, FP:false positive, and FN: False negative }\end{array}$} \\
\hline
\end{tabular}


TABLE 2 - continued from the previous page

\begin{tabular}{|c|c|c|c|c|}
\hline Study & $\begin{array}{c}\text { Data Description } \\
\text { \& AL } \\
\end{array}$ & Approach & Speed & $\begin{array}{c}\text { Validation } \\
\text { and Accuracy }\end{array}$ \\
\hline $\mathrm{OM}$ & \multicolumn{4}{|c|}{ denotes overlap measure: $\mathrm{OM}=\frac{\mathrm{TP}}{\mathrm{TP}+\mathrm{FP}+\mathrm{FN}}$} \\
\hline SEN: & \multicolumn{4}{|c|}{ denotes the sensitivity: $\mathrm{SEN}=\frac{\mathrm{TP}}{\mathrm{TP}+\mathrm{FN}}$. } \\
\hline SPE: & \multicolumn{4}{|c|}{ denotes the specificity: $\mathrm{SPE}=\frac{\mathrm{TN}}{\mathrm{TN}+\mathrm{FP}}$. } \\
\hline PPV: & \multicolumn{4}{|c|}{ denotes the positive predictive value: $\mathrm{PPV}=\frac{\mathrm{TP}}{\mathrm{TP}+\mathrm{FP}}$} \\
\hline ACC: & \multicolumn{4}{|c|}{ Accuracy: $\mathrm{ACC}=\frac{\mathrm{TP}+\mathrm{TN}}{\mathrm{TP}+\mathrm{FP}+\mathrm{FN}+\mathrm{TN}}$} \\
\hline RMSD & \multicolumn{4}{|c|}{ denotes the root mean square difference of the distance between } \\
\hline AD: & \multicolumn{4}{|c|}{ denotes the mean absolute surface distance. } \\
\hline HD: & \multicolumn{4}{|c|}{ denotes the Hausdorff distance: the mean maximum distance } \\
\hline ПШ. & \multicolumn{4}{|c|}{ of a set to nearest point in the other set. } \\
\hline SPD: & \multicolumn{4}{|c|}{ denotes the mean signed border positioning error } \\
\hline SCD: & \multicolumn{4}{|c|}{ denotes symmetrical point-to-mesh comparisons error. } \\
\hline
\end{tabular}

\section{Detection of lung nodules}

After the definition of the search space for the nodules (e.g., the segmented lung fields), nodule detection is the next step in lung cancer CAD systems. Early detection of lung tumors (visible on chest film as nodules) may increase the patients chance of survival [68], but the nodule detection problem is a complicated task; see, e.g., [69, 70]. Nodules show up as relatively low-contrast white circular objects within the lung fields. The difficulty for CAD schemes is to distinguish true nodules from (overlapping) shadows, vessels and ribs. At present, spiral LDCT is of prime interest for screening (high risk) groups for early detection of lung cancer [71-73]. The LDCT provides chest scans with very high spatial, temporal, and contrast resolution of anatomic structures and is able to gather a complete 3D volume of a human thorax in a single breath-hold [69]. Hence, for these reasons, recently most lung cancer screening programs are being investigated in the United States [73-77] and Japan [70,72,78,79] with helical LDCT as the screening modality of choice.

The CAD schemes for detection of lung nodules in thoracic CT (i.e., CADe) generally consists of two major stages: (1) initial candidate nodules are selected (i.e, identification of nodule candidates) and then (2) the false positive nodules (FPNs), are partially 
eliminated while preserving the true ones (TPNs) (i.e., classification of the identified nodule candidates into nodules or non-nodules (i.e., normal anatomic structures)). At the first stage, conformal nodule filtering [80] or unsharp masking [81] can enhance nodules and suppress other structures to separate the candidates from the background by simple thresholding (to improve the separation, background trend is corrected in [82-85] within image regions of interest) or multiple gray-level thresholding technique $[38,86,87]$. A series of 3D cylindrical and spherical filters are used to detect small lung nodules from high resolution CT images [88-92]. Circular and semicircular nodule candidates can be detected by template matching $[81,93,94]$. However, these spherical, cylindrical, or circular assumptions are not adequate for describing general geometry of the lesions. This is because their shape can be irregular due to the spiculation or the attachments to the pleural surface (i.e., juxtapleural and peripheral) and vessels (i.e.,vascularized) [95]. In [96-99], they used morphological operators to detect lung nodules. The drawbacks to these approaches are the difficulties in detecting lung wall nodules. Also, there are other pattern recognition techniques used in detection of lung nodules such as clustering [100-103], linear discriminate functions [104], rule-based classification [105], Hough transform [106], connected component analysis of thresholded CT slices [107, 108], gray level distance transform [102], and patient-specific a priori model [109]. The FPNs are excluded at the second stage by nodule classification [82, 83, 106, 110-112].

The most popular way to do classification is to use a feature-based classifier. First, the nodule candidates identified in the first step are segmented, and features are extracted from the segmented nodule candidates. Features may include morphologic (or shapebased) features (e.g., size, circularity [83], curvature [112], etc.), gray-level-based features (including histogram-based features), and texture features. The task of the classifier is to determine "optimal" boundaries for separating classes (i.e., nodules or non-nodules) in the multi-dimensional feature space which is formed by the input features [113]. Featurebased classifiers include linear discriminant analysis (LDA) [114], rule-based or linear clas- 
sifier [38, 85, 86, 88, 90, 100,103, 115]; template matching [109]; nearest cluster [97, 99]; Markov random field [116], quadratic discriminant analysis [114], multilayer perceptron (often called just an artificial neural network; ANN) [96, 111, 117-119], and a support vector machine (SVM) $[120,121]$. A classifier is trained with sets of input features and correct class labels. A class label of 1 is assigned to the corresponding output unit when a training sample belongs to that class, and 0 is assigned to the other output units. After training, the class of the unit with the maximum value is determined to be the corresponding class to which an unknown sample belongs.

Recently, as available computational power increased dramatically, pixel/voxelbased machine learning (PML) [122] emerged in medical image analysis which uses pixel/voxel values in images directly instead of features calculated from segmented regions as input information; thus, feature calculation or segmentation is not required. Because the PML can avoid errors caused by inaccurate feature calculation and segmentation which often occur for subtle or complex lesions, the performance of the PML can potentially be higher for such lesions than that of common feature-based classifiers. PML includes neural filters [123,124], convolution neural networks (NNs) [125-129](including shiftinvariant NNs [130-132]), and massive-training ANNs (MTANNs) [133-136] (including multiple MTANNs [133,137-139], a mixture of expert MTANNs [140,141], a Laplacian eigenfunction MTANN (LAP-MTANN) [142], and a massive-training support vector regression (MTSVR) [143]). Convolution NNs have been applied for classification tasks such as false-positive (FP) reduction in CADe schemes for detection of lung nodules in chest radiographs [125-127], FP reduction in CADe schemes for detection of microcalcifications [128] and masses [129] in mammography. MTANNs have been used for classification, such as FP reduction in CADe schemes for detection of lung nodules in chest radiographs [137] and thoracic CT [133, 138, 144], distinction between benign and malignant nodules in thoracic CT [139], and FP reduction in a CADe scheme for polyp detection in CT colonography [134, 140-143]. 
Technical development of the classification step in CADe schemes for detection of lung nodules in CT is summarized in Table 3. Giger et al. [145] developed a CADe scheme for detection of lung nodules in CT. In their CADe scheme, classification was performed by geometric feature analysis in conjunction with a comparison of suspected regions in each slice with suspected regions in adjacent slices. Armato et al. $[38,146]$ extended the method to include 3D feature analysis, a rule-based scheme, and LDA for classification. Gurcan et al. [100] employed a similar approach, i.e., a rule-based scheme based on 2D and 3D features followed by LDA for classification. Lee et al. [93] employed a simpler approach which is a rule-based scheme based on 13 features for classification. Ko et al. [86] differentiated between normal structures (vessels and bronchi) and nodules by the analysis of the candidates location and shape using a rule based classifier. Their method was able to detect nodules with diameter larger than $3 \mathrm{~mm}$ and missed those with diameter less than $3 \mathrm{~mm}$ or contacted the lung border. Kanazawa et al. [103] segmented the nodule candidates (normal structures (vessels and bronchi) and nodules) within the lung fields using a fuzzy clustering method [147]. For each candidate, they extracted a shape, a gray-level, and a position features. Then, a rule based filter was used to combine these features in order to detect the lung nodules. Brown et al. [109] build semantic network priori models to describe the lung nodules and other structures. In the training phase, a set of features, composed of the $\mathrm{X}$-ray attenuation range, the relative location, the volume, and a sphericity sphericity shape parameter, were used in the semantic network nodes to describe the expectation models for the lung nodules as well as other structures. For each test candidate, a fuzzy logic was used to score the match between the extracted candidate features and the priori estimated models to define its belonging to either nodule or other structures. Wiemker et al. [94] evaluated the compactness, thickness of connecting vessels, average Hounsfield (HU) value, and HU distribution within the nodule candidate to detect nodules using $1 \mathrm{~mm}$ high resolution (HR) CT slices. On 12 HRCT exams with 203 nodules, their method achieved a sensitivity of $0.86 \%$ and 4.4 FPs per case for nodules with diameter $\geq 1 \mathrm{~mm}$. Mekada et al. [85] discrim- 
inated between nodule regions and normal structures (e.g., vessels) using the maximum distance inside connected components (MDCC) in 3D X-ray CT images. The number of false positive nodules was reduced by applying a minimum directional difference filter for the nodule candidates that has size smaller than a given threshold value. Their method achieved a sensitivity of $71 \%$ with an average number of 7.4 false positive per case in a study composed of 242 CT medical exams. Awai et al. [96] identified the initial potential nodules using a sieve filter that selected the intrapulmonary structures larger than a predefined size as lung nodule candidates. Then, an ANN classifier was used to determine if the lesion is true nodule or not based on a set of extracted candidate features, including the volume, roundness, average diameter, maximum diameter and the diameter perpendicular to it, and distance between potential nodule and thoracic. The sensitivity of this method was $80 \%$ and 0.87 FPs nodule per section on a test group composed of 82 CT exams (3556 sections) containing 78 nodules. Paik et al. [91] used a method, called the surface normal overlap method (SNO), to detect the lung nodules and colon polyps. The normal overlap method (SNO) describes the shape and geometry of a potential nodule and assigns a score for each shape. A threshold score was used to discriminate between the lesions and other structures. Their method was tested on eight lung datasets, achieving a varying sensitivity based on the allowed false positive per sets. At 1.3 FPs per dataset, a sensitivity of $80 \%$ was achieved; at 5.6 FPs per dataset a sensitivity of 90\% was achieved, and at 165 FPs per dataset; and a sensitivity of $100 \%$ was achieved. Mendonça et al. [92] used a filter for highlighting the nodule-like structures (ROI) in CT images. For every voxel in the ROI, the eigenvalues of a curvature tensor were computed and thresholds derived from anatomical models (i.e, a geometric and an intensity models) were used to label each voxel as spherical (e.g., nodules), cylindrical (e.g., vessels), or neither. Suzuki et al. [133] developed an MTANN for reduction of a single source of FPs and a multiple MTANN scheme for reduction of multiple sources of FPs that had not been removed by LDA. This MTANN approach did not require a large number of training cases: the MTANN was able to be 
trained with 10 positive and 10 negative cases [148-150], whereas feature-based classifiers generally require 400-800 training cases [148-150]. Arimura et al. [138] employed a rule-based scheme followed by LDA or the MTANN [133] for classification. Farag et al. [151] developed a template modeling approach using level sets for classification. Ge et al. [152] incorporated 3D gradient field descriptors and ellipsoid features in LDA for classification. Matsumoto et al. [153] employed LDA with 8 features for classification. Yuan et al. [154] tested a commercially available CADE system (ImageChecker CT, LN-1000, by R2 Technology, Sunnyvale, CA; Hologic now). Pu et al. [155] developed a scoring method based on the similarity distance of medial axis-like shapes obtained through a progressive clustering strategy combined with a marching cube algorithm from a sphere based shape. Retico et al. [156] used the MTANN approach (they call it in their paper) for classification. Ye et al. [44] used a rule-based scheme followed by a weighted SVM for classification. Golosio et al. [157] used a fixed-topology ANN for classification and they evaluated their CADe scheme with a publicly available database from the Lung Image Database Consortium (LIDC) [158]. Murphy et al. [159] used a k-nearest-neighbor classifier for classification. Tan et al. [160] developed a feature-selective classifier based on a genetic algorithm and ANNs for classification. Messay et al. [161] developed a sequential forward selection process for selecting the optimum features for LDA and quadratic discriminant analysis. Riccard et al. [162] used a heuristic approach based on geometric features followed by an SVM for classification. Thus, various approaches have been proposed for the classification component in CADe schemes.

This overview shows that some important factors should have further investigation in designing any CADe system for detecting the lung nodule including the automation level, the speed, the ability of the detection scheme to detect nodules of different shapes, e.g., irregular shape nodules not only the spherical ones, and the ability of the CADe system to detect cavity nodules, nodules contacted to the lung borders, and small nodules (e.g., less than $3 \mathrm{~mm}$ ). 
TABLE 3: Classification component in CADe schemes. Studies are ordered by their publication year

\begin{tabular}{|c|c|c|c|c|}
\hline Study & Feature/Input & Classifier/Method & Database & Performance \\
\hline $\begin{array}{l}\text { Giger et } \\
\text { al. [145] }\end{array}$ & $\begin{array}{l}\text { Geometric } \\
\text { features }\end{array}$ & $\begin{array}{l}\text { Comparison } \\
\text { of geometric } \\
\text { features }\end{array}$ & $\begin{array}{l}\text { Thick-slice diagnostic CT } \\
\text { scans of } 8 \text { patients with } 47 \\
\text { nodules }\end{array}$ & $\begin{array}{l}\text { Sensitivity of } 94 \% \\
\text { with1.25 FPs } \\
\text { case }\end{array}$ \\
\hline $\begin{array}{l}\text { Kanazawa } \\
\text { et } \\
\text { al. }[103]\end{array}$ & 8 features & $\begin{array}{l}\text { Rule based } \\
\text { scheme }\end{array}$ & $\begin{array}{l}\text { Helical CT scans from } 450 \\
\text { patients with } 230 \text { nodules } \\
\text { (a total of } 15,750 \text { image } \\
\text { sections) }\end{array}$ & Sensitivity of $90 \%$ \\
\hline $\begin{array}{l}\text { Armato } \\
\text { et al. [38, } \\
146]\end{array}$ & $\begin{array}{l}\text { Nine } 2 \mathrm{D} \text { and } \\
3 \mathrm{D} \text { features }\end{array}$ & $\begin{array}{l}\text { Rule-based } \\
\text { scheme and LDA }\end{array}$ & $\begin{array}{l}\text { Thick-slice }(10 \mathrm{~mm}) \text { diag- } \\
\text { nostic CT scans of } 43 \mathrm{pa}- \\
\text { tients with } 171 \text { nodules }\end{array}$ & $\begin{array}{l}\text { Sensitivity of } 70 \% \\
\text { with } 42.2 \text { FPs per } \\
\text { case in a leave-one- } \\
\text { out test }\end{array}$ \\
\hline $\begin{array}{l}\text { Lee et } \\
\text { al. [93] }\end{array}$ & 13 features & $\begin{array}{l}\text { Rule-based } \\
\text { scheme and LDA }\end{array}$ & $\begin{array}{l}\text { Thick-slice }(10 \mathrm{~mm}) \text { diag- } \\
\text { nostic CT scans of } 20 \text { pa- } \\
\text { tients with } 98 \text { nodules }\end{array}$ & 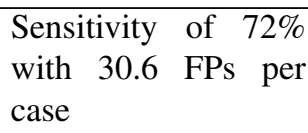 \\
\hline $\begin{array}{l}\text { Ko et } \\
\text { al. [86] }\end{array}$ & $\begin{array}{l}\text { Location } \\
\text { and } 2 \text { shape } \\
\text { features (cir- } \\
\text { cularity and } \\
\text { roundness) }\end{array}$ & $\begin{array}{l}\text { Rule-based } \\
\text { scheme }\end{array}$ & $\begin{array}{l}\text { Helical CT scans of } 16 \\
\text { studies (8 initial and } 8 \\
\text { follow-up) obtained from } 8 \\
\text { patients with } 370 \text { nodules }\end{array}$ & Sensitivity of $86 \%$ \\
\hline $\begin{array}{l}\text { Brown et } \\
\text { al. [109] }\end{array}$ & $\begin{array}{l}\text { Prior models } \\
\text { based on } 4 \\
\text { features }\end{array}$ & Fuzzy matching & $\begin{array}{l}\text { Thick slice }(5-10 \mathrm{~mm}) \mathrm{CT} \\
\text { scans of } 17 \text { patients with } \\
36 \text { nodules }\end{array}$ & $\begin{array}{l}\text { Sensitivity of } 86 \% \\
\text { and } 11 \text { FPs per case. }\end{array}$ \\
\hline $\begin{array}{l}\text { Wiemker } \\
\text { et al. }[94]\end{array}$ & $\begin{array}{l}4 \text { shape and } \\
\text { intensity fea- } \\
\text { tures }\end{array}$ & $\mathrm{NA}$ & $\begin{array}{l}\text { Thin-slice }(1 \mathrm{~mm}) \text { HRCT } \\
\text { scans of } 50 \text { subjects (A to- } \\
\text { tal of more than } 20,000 \\
\text { image sections), } 12 \text { scans } \\
\text { were reviewed by radiolo- } \\
\text { gist with } 203 \text { nodules }\end{array}$ & $\begin{array}{l}\text { Sensitivity of } 86 \% \\
\text { with } 4.9 \text { FPs per } \\
\text { case for nodules with } \\
\text { diameter } \geq 1 \mathrm{~mm} \text { and } \\
\text { sensitivity of } 95 \% \\
\text { with } 4.4 \text { FPs per case } \\
\text { with diameters } \geq \\
2 \mathrm{~mm}\end{array}$ \\
\hline $\begin{array}{l}\text { Gurcan et } \\
\text { al. }[100]\end{array}$ & $\begin{array}{l}\text { Six 2D and } \\
\text { 3D features }\end{array}$ & $\begin{array}{l}\text { Rule-based } \\
\text { scheme and LDA }\end{array}$ & $\begin{array}{l}\text { Thick-slice }(2.5-5 \mathrm{~mm} \text {, } \\
\text { mostly } 5 \mathrm{~mm}) \text { diagnostic } \\
\text { CT scans of } 34 \text { patients } \\
\text { with } 63 \text { nodules }\end{array}$ & $\begin{array}{l}\text { Sensitivity of } 84 \% \\
\text { with } 74.4 \text { FPs per } \\
\text { case in a leave-one- } \\
\text { out test }\end{array}$ \\
\hline $\begin{array}{l}\text { Suzuki et } \\
\text { al. [133] }\end{array}$ & $\begin{array}{l}\text { Pixel values } \\
\text { in a 9x9 } \\
\text { subregion }\end{array}$ & $\begin{array}{l}\text { Multiple } \\
\text { MTANNs }\end{array}$ & $\begin{array}{l}\text { Thick-slice }(10 \mathrm{~mm}) \\
\text { screening LDCT scans of } \\
63 \text { patients with } 71 \text { nod- } \\
\text { ules with solid, part-solid } \\
\text { and non-solid patterns, } \\
\text { including } 66 \text { cancers }\end{array}$ & $\begin{array}{l}\text { Sensitivity of } 80.3 \% \\
\text { with } 4.8 \text { FPs per case } \\
\text { in a validation test }\end{array}$ \\
\hline $\begin{array}{l}\text { Mekada } \\
\text { et al. }[85]\end{array}$ & $\begin{array}{l}\text { Minimum di- } \\
\text { rectional dif- } \\
\text { ference filter }\end{array}$ & $\begin{array}{l}\text { Rule } \\
\text { scheme }\end{array}$ & $\begin{array}{l}\text { CT scans of } 7 \text { subjects } \\
\text { (6 patients and } 1 \text { normal } \\
\text { subject) with } 361 \text { nodules } \\
(160-350 \text { sections per case) }\end{array}$ & $\begin{array}{l}\text { Sensitivity of } 71 \% \\
\text { and } 7.4 \text { FPs per case }\end{array}$ \\
\hline
\end{tabular}


TABLE 3 - continued from the previous page

\begin{tabular}{|c|c|c|c|c|}
\hline Study & Feature/Input & Classifier/Method & Database & Performance \\
\hline $\begin{array}{l}\text { Arimura } \\
\text { et } \\
\text { al. }[138]\end{array}$ & $\begin{array}{l}\text { Pixel values } \\
\text { in a 9x9 } \\
\text { subregion for } \\
\text { MTANNs } \\
\text { (Selected } \\
\text { features for } \\
\text { LDA) }\end{array}$ & $\begin{array}{l}\text { Rule-based } \\
\text { scheme followed } \\
\text { by multiple } \\
\text { MTANNs (or } \\
\text { LDA with Wilks' } \\
\text { lambda stepwise } \\
\text { feature selection) }\end{array}$ & $\begin{array}{l}106 \text { thick-slice }(10 \mathrm{~mm}) \\
\text { screening LDCT scans of } \\
73 \text { patients with } 109 \text { can- } \\
\text { cers with solid, part-solid } \\
\text { and non-solid patterns }\end{array}$ & $\begin{array}{l}\text { Sensitivity of } 83 \% \\
\text { with 5.8 FPs per case } \\
\text { in a validation test (or } \\
\text { a leave-one-patient- } \\
\text { out test for LDA) }\end{array}$ \\
\hline $\begin{array}{l}\text { Awai et } \\
\text { al. [96] }\end{array}$ & $\begin{array}{l}6 \text { geometric } \\
\text { features }\end{array}$ & $\begin{array}{l}\text { Artificial neural } \\
\text { network classier }\end{array}$ & $\begin{array}{l}\text { CT scans of } 82 \text { patients } \\
\text { with } 78 \text { nodules (a total of } \\
3,556 \text { image sections) }\end{array}$ & $\begin{array}{ll}\text { Sensitivity } & \text { of } 80 \% \\
\text { with } 0.87 & \text { FPs per } \\
\text { section } & \end{array}$ \\
\hline $\begin{array}{l}\text { Paik et } \\
\text { al. }[91]\end{array}$ & $\begin{array}{l}\text { Surface } \\
\text { normal over- } \\
\text { lap (SNO) } \\
\text { method that } \\
\text { describes the } \\
\text { shape and } \\
\text { geometry }\end{array}$ & $\begin{array}{l}\text { Rule-based } \\
\text { scheme }\end{array}$ & CT scans of 8 patients & $\begin{array}{l}\text { Sensitivity of } 90 \% \\
\text { with } 5.6 \text { FPs per case } \\
\text { in a cross validation } \\
\text { test }\end{array}$ \\
\hline $\begin{array}{l}\text { Farag et } \\
\text { al. }[151]\end{array}$ & NA & $\begin{array}{l}\text { Template model- } \\
\text { ing approach us- } \\
\text { ing level sets }\end{array}$ & $\begin{array}{l}\text { Thin-slice }(2.5 \quad \mathrm{~mm}) \\
\text { screening LDCT } \text { scans } \\
\text { of } 16 \text { patients with } 119 \\
\text { nodules and } 34 \text { normal } \\
\text { patients }\end{array}$ & $\begin{array}{l}\text { Sensitivity of } 93 \% \\
\text { with XX FPs per case }\end{array}$ \\
\hline $\begin{array}{l}\text { Ge et } \\
\text { al. }[152]\end{array}$ & $\begin{array}{l}44 \text { features } \\
\text { including 3D } \\
\text { gradient field } \\
\text { descriptors } \\
\text { and ellipsoid } \\
\text { features }\end{array}$ & $\begin{array}{l}\text { LDA with Wilks' } \\
\text { lambda stepwise } \\
\text { feature selection }\end{array}$ & $\begin{array}{l}82 \text { thin-slice }(1.0-2.5 \mathrm{~mm}) \\
\text { CT scans of } 56 \text { patients } \\
\text { with } 116 \text { solid nodules }\end{array}$ & $\begin{array}{l}\text { Sensitivity of } 80 \% \\
\text { with } 14.7 \text { FPs per } \\
\text { case in a leave-one- } \\
\text { patient-out test }\end{array}$ \\
\hline $\begin{array}{l}\text { Mendonça } \\
\text { et al. [92] }\end{array}$ & $\begin{array}{l}\text { Geometric } \\
\text { and inten- } \\
\text { sity models } \\
\text { combined } \\
\text { with eigen } \\
\text { curvature } \\
\text { analysis }\end{array}$ & $\begin{array}{l}\text { Rule based } \\
\text { scheme }\end{array}$ & $\begin{array}{l}\text { Thin slice (1.25 and } 2.5 \\
\text { mm) CT scans of } 242 \text { ex- } \\
\text { ams from two institutions: } \\
50 \text { CT scans with } 109 \text { nod- } \\
\text { ule and } 192 \text { CT scans with } \\
210 \text { nodules }\end{array}$ & $\begin{array}{l}\text { Sensitivity of } 67.5 \% \\
\text { and } 9.3 \text { FPs per case } \\
\text { for data from the first } \\
50 \mathrm{CT} \text { scans and sen- } \\
\text { sitivity of } 62.9 \% \text { and } \\
10.3 \text { FPs per case for } \\
\text { the second } 192 \mathrm{CT} \\
\text { scans in a leave-one- } \\
\text { out test }\end{array}$ \\
\hline $\begin{array}{l}\text { Matsumoto } \\
\text { et } \\
\text { al. }[153]\end{array}$ & 8 features & LDA & $\begin{array}{l}\text { Thick-slice (5 or } 7 \mathrm{~mm}) \\
\text { diagnostic CT scans of } 5 \\
\text { patients ( } 4 \text { of which used } \\
\text { contrast media) with } 50 \\
\text { nodules }\end{array}$ & $\begin{array}{l}\text { Sensitivity of } 90 \% \\
\text { with } 64.1 \text { FPs per } \\
\text { case in a leave-one- } \\
\text { out test }\end{array}$ \\
\hline $\begin{array}{l}\text { Yuan et } \\
\text { al. [154] }\end{array}$ & NA & $\begin{array}{l}\text { ImageChecker } \\
\text { CT LN-1000 by } \\
\text { R2 Technology }\end{array}$ & $\begin{array}{l}\text { Thin-slice }(1.25 \mathrm{~mm}) \mathrm{CT} \\
\text { scans of } 150 \text { patients with } \\
628 \text { nodules }\end{array}$ & $\begin{array}{l}\text { Sensitivity of } 73 \% \\
\text { with } 3.2 \text { FPs per case } \\
\text { in an independent } \\
\text { test }\end{array}$ \\
\hline \multicolumn{5}{|c|}{ continued on the next page ... } \\
\hline
\end{tabular}


TABLE 3 - continued from the previous page

\begin{tabular}{|c|c|c|c|c|}
\hline Study & Feature/Input & Classifier/Method & Database & Performance \\
\hline $\begin{array}{l}\text { Pu et } \\
\text { al. [155] }\end{array}$ & NA & $\begin{array}{l}\text { Dcoring method } \\
\text { based on the sim- } \\
\text { ilarity distance } \\
\text { combined with a } \\
\text { marching cube } \\
\text { algorithm }\end{array}$ & $\begin{array}{l}\text { Thin-slice }(2.5 \quad \mathrm{~mm}) \\
\text { screening CT scans of } 52 \\
\text { patients with } 184 \text { nodules } \\
\text { including } 16 \text { non-solid } \\
\text { nodules }\end{array}$ & $\begin{array}{l}\text { Sensitivity of } 81.5 \% \\
\text { with } 6.5 \text { FPs per case }\end{array}$ \\
\hline $\begin{array}{l}\text { Retico et } \\
\text { al. }[156]\end{array}$ & $\begin{array}{l}\text { Pixel val- } \\
\text { ues in a } \\
\text { subvolume }\end{array}$ & $\begin{array}{l}\text { Voxel-based } \\
\text { neural approach } \\
\text { (MTANN) }\end{array}$ & $\begin{array}{l}\text { Thin-slice }(1 \mathrm{~mm}) \text { screen- } \\
\text { ing CT scans of } 39 \text { patients } \\
\text { with } 102 \text { nodules }\end{array}$ & $\begin{array}{l}\text { Sensitivities of } 80- \\
85 \% \text { with } 10-13 \text { FPs } \\
\text { per case }\end{array}$ \\
\hline $\begin{array}{l}\text { Ye et } \\
\text { al. [44] }\end{array}$ & 15 features & $\begin{array}{l}\text { Rule-based } \\
\text { scheme followed } \\
\text { by a weighted } \\
\text { SVM }\end{array}$ & $\begin{array}{l}\text { Thin-slice }(1 \mathrm{~mm}) \text { screen- } \\
\text { ing CT scans of } 54 \text { patients } \\
\text { with } 118 \text { nodules including } \\
17 \text { non-solid nodules }\end{array}$ & $\begin{array}{l}\text { Sensitivity of } 90.2 \% \\
\text { with } 8.2 \text { FPs per case } \\
\text { in an independent } \\
\text { test }\end{array}$ \\
\hline $\begin{array}{l}\text { Golosio et } \\
\text { al. [157] }\end{array}$ & $\begin{array}{l}42 \text { features } \\
\text { from mul- } \\
\text { tithreshold } \\
\text { ROI }\end{array}$ & $\begin{array}{l}\text { Fixed-topology } \\
\text { ANN }\end{array}$ & $\begin{array}{l}\text { Thin-slice }(1.5-3.0 \mathrm{~mm}) \\
\text { CT scans of } 83 \text { patients } \\
\text { with } 148 \text { nodules that one } \\
\text { radiologist detected from } \\
\text { LIDC database }\end{array}$ & $\begin{array}{l}\text { Sensitivity of } 79 \% \\
\text { with } 4 \text { FPs per case in } \\
\text { an independent test }\end{array}$ \\
\hline $\begin{array}{l}\text { Murphy } \\
\text { et } \\
\text { al. [159] }\end{array}$ & $\begin{array}{l}\text { Features se- } \\
\text { lected from } \\
135 \text { features }\end{array}$ & $\begin{array}{l}\text { k-nearest- } \\
\text { neighbor classi- } \\
\text { fier }\end{array}$ & $\begin{array}{l}\text { Thin-slice screening CT } \\
\text { scans of } 813 \text { patients with } \\
1,525 \text { nodules }\end{array}$ & $\begin{array}{l}\text { Sensitivity of } 80 \% \\
\text { with } 4.2 \text { FPs per case } \\
\text { in an independent } \\
\text { test }\end{array}$ \\
\hline $\begin{array}{l}\text { Messay et } \\
\text { al. [161] }\end{array}$ & $\begin{array}{l}\text { Features se- } \\
\text { lected from } \\
245 \text { features }\end{array}$ & $\begin{array}{lr}\text { LDA } & \text { and } \\
\text { quadratic } & \text { dis- } \\
\text { criminant analy- } \\
\text { sis with feature } \\
\text { selection }\end{array}$ & $\begin{array}{l}\text { Thin-slice CT scans of } 84 \\
\text { patients with } 143 \text { nodules } \\
\text { from LIDC database }\end{array}$ & $\begin{array}{l}\text { Sensitivity of } 83 \% \\
\text { with } 3 \text { FPs per case } \\
\text { in a 7-fold cross- } \\
\text { validation test }\end{array}$ \\
\hline $\begin{array}{l}\text { Tan et } \\
\text { al. }[160]\end{array}$ & $\begin{array}{l}45 \text { initial fea- } \\
\text { tures }\end{array}$ & \begin{tabular}{lr}
\multicolumn{2}{l}{ Feature-selective } \\
classifier $r$ based \\
on a genetic \\
algorithm $r$ and \\
ANNs
\end{tabular} & $\begin{array}{l}\text { Thin-slice CT scans of } 125 \\
\text { patients with } 80 \text { nodules } \\
\text { that } 4 \text { radiologists agreed } \\
\text { from LIDC database }\end{array}$ & $\begin{array}{l}\text { Sensitivity of } 87.5 \% \\
\text { with } 4 \text { FPs per case in } \\
\text { an independent test }\end{array}$ \\
\hline $\begin{array}{l}\text { Riccard } \\
\text { et } \\
\text { al. }[162]\end{array}$ & $\begin{array}{l}\text { Maximum in- } \\
\text { tensity projec- } \\
\text { tion data from } \\
\text { volume of in- } \\
\text { terest }\end{array}$ & 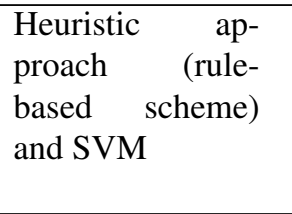 & $\begin{array}{l}\text { Thin-slice CT scans of } 154 \\
\text { patients with } 117 \text { nodules } \\
\text { that } 4 \text { radiologists agreed } \\
\text { from LIDC database }\end{array}$ & $\begin{array}{l}\text { Sensitivity of } 71 \% \\
\text { with } 6.5 \text { FPs per case } \\
\text { in a } 2 \text {-fold cross vali- } \\
\text { dation test }\end{array}$ \\
\hline $\begin{array}{l}\text { ANN: } \\
\text { CT: } \\
\text { FP: } \\
\text { HRCT: } \\
\text { LDA: } \\
\text { LDCT: } \\
\text { LIDC: } \\
\text { MTANN: } \\
\text { NA: } \\
\text { ROI: } \\
\text { SNO: } \\
\text { SVM }\end{array}$ & $\begin{array}{l}\text { denotes artificia } \\
\text { denotes comput } \\
\text { denotes false pc } \\
\text { denotes high re } \\
\text { denotes linear } \\
\text { denotes low do } \\
\text { denotes lung im } \\
\text { denotes massiv } \\
\text { denotes not app } \\
\text { denotes region- } \\
\text { denotes surface } \\
\text { denotes suppor }\end{array}$ & $\begin{array}{l}\text { neural network } \\
\text { d tomography } \\
\text { itive } \\
\text { lution computed tom } \\
\text { scriminant analysis } \\
\text { computed tomograp } \\
\text { ge database consorti } \\
\text { training artificial neu } \\
\text { cable } \\
\text { f-interest. } \\
\text { ormal overlap } \\
\text { vector machine }\end{array}$ & $\begin{array}{l}\text { y } \\
\text { al network }\end{array}$ & \\
\hline
\end{tabular}




\section{Lung Nodule Segmentation}

Lung nodule segmentation refers to a task of delineating the spatial extent of focal nodular lesions appearing in chest $\mathrm{CT}$ scans, providing a critical foundation of CAD systems for lung cancers [163-165]. The nodule segmentation is a very important and crucial step in many lung cancer applications. In this section, the clinical applications of lung nodule segmentation are outlined. Then, a review of the state-of-the-art segmentation techniques of the lung nodules from CT images as well as from PET images is provided. Finally, various aspects of challenges that face the techniques developed for the nodule segmentation problem will be addressed.

\section{Clinical Applications}

Accurate nodule segmentation is a crucial prerequisite for various diagnostic and treatment procedures for lung cancer [166], such as diagnosing tumor growth in followup CTs $[167,168]$, monitoring tumor response to therapy $[169,170]$, computer-aided lung cancer screening for early detection $[93,109,171]$, and computer-aided diagnosis of tumor malignancy $[137,172]$. In this application context, segmentation accuracy directly influences important clinical factors, such as the minimum size of measurable lesions and the shortest time duration for repeat CT in follow-up studies. Another interesting approach is to derive the standard RECIST/WHO 2D measures of lesions from the results of their volumetric 3D segmentation in order to improve their accuracy and reproducibility $[173,174]$.

The segmentation also defines a local image area, from which image features can be extracted for further computational analyses. For example, lung cancer screening by CADe $[93,109,171]$ often enhances the overall detection accuracy by segmenting detected nodules as a post-analysis to remove false-positive cases [175]. Malignancy classification of lung nodules in CADx [172] will also rely on accurate segmentation for extracting image appearance features whose quality dictates the overall classification performance [176]. 
Thus improving accuracy of nodule segmentation has a direct impact to these clinical tasks. While segmentation of large solitary nodule can be straightforward, there exists types of nodules, such as small or part-solid nodules, which pose difficulty in accurate segmentation. Because these difficult cases are also of clinical importance (e.g., early detection of lung cancer with small nodules [177]; part-solid nodule with high likelihood of being malignant [178-180]), nodule segmentation plays a critical role in successfully administering these clinical tasks.

\section{CT Nodules Segmentation Techniques}

Due to the increasing clinical significance described above, the number of papers reported in the literature of pulmonary nodule segmentation has been increasing rapidly. The advent of high-resolution thin-slice and multi-detector CT technologies in early 2000's has shifted trends in nodule segmentation research from early thresholding-based 2D methods to more sophisticated flexible 3D/volumetric segmentations. Prior to the advent of CT in routine medical practices, automatic detection, segmentation and analysis of nodules in $2 \mathrm{D}$ chest radiography were actively investigated $[181,182]$. Segmentation algorithms proposed in this context were intrinsically 2D-based. During the early phase of CT applications, images are often made with a large slice thickness. Some early methods in the literature $[183,184]$ have also adopted this $2 \mathrm{D}$ approach for this reason. This section summarizes the advances in nodule segmentation focusing on the recent volumetric approaches.

Technical approaches previously reported for volumetric lung nodule segmentation can be roughly classified into the following eleven categories: 1) thresholding [167, 168, 183-187], 2) mathematical morphology [95,98, 188-191], 3) region growing [174, 189, 190, 192-194], 4) deformable model [195-201], 5) dynamic programming [202-204], 6) spherical/ellipsoidal model fitting [205-209] 7) probabilistic classification [119,210215], 8) discriminative classification [216,217], 9) mean shift [207, 218,219], 10) graphcuts [220,221], and 11) watersheds [222]. Next, an overview of the technical approaches 
for lung nodule segmentation is given.

a. Thresholding (TH) is one of the most ubiquitous and straightforward methods for solving general segmentation problem. It yields a binary (foreground/background) segmentation of volume of interest (VOI) by labeling each voxel by testing whether its intensity value surpasses a specific threshold value or not [45]. This approach was adapted by early methods proposed by Zhao et al. [183, 185] and Yankelevitz et al. [167, 184, 186]. Automatic data-driven methods to determine threshold values have been proposed by using K-mean clustering $[167,184]$ and average gradient magnitudes and boundary compactness $[183,185]$.

b. Mathematical morphology (MM) is another popular technique in lung nodule segmentation especially for handling special cases that attached to non-target structures such as vessels (juxtavascular) and parenchymal wall or diaphragm (juxtapleural). MM is a set theoretic technique for processing geometric structures in binary and grayscale images [45]. It offers various morphological operations (MO) with four basic operators (erosion, dilation, opening and closing) with a task-specific structuring element. Commonly, a sequence of iterative MOs are used to remove non-target structures juxtaposed to the target nodule in an initial binary segmentation result. Kostis et al. [95, 188] and Kuhnigk et al. $[189,190]$ have proposed effective iterative approaches for binary morphological filtering with various combinations of these basic operators. Okada et al. [191] presented a data-driven method to determine the ellipsoidal structuring element from anisotropic Gaussian fitting. Grayscale MOs have also been successfully applied to nodule segmentation. Fetita et al. [98] proposed an approach with SMDC connection cost for handling the juxtaposed cases.

c. Region growing (RG) is another classical image segmentation method that has been successfully adapted to the lung nodule segmentation problem. It identifies a connected-component region that surrounds a seeded pixel by iteratively adding neighboring pixels which satisfies a logical predicate defining pixel intensity proximity [45]. 
RG has been popular among recent methods as their base component to produce initial rough segmentation to be improved on further, replacing the simpler $\mathrm{TH}$ adopted by earlier methods in the same context $[167,183-186]$. In the MM-based approach by Kuhnigk et al. $[189,190]$, RG was adopted in this manner. There are more recent studies $[174,192-$ 194] that have extended this approach as the main component of their overall segmentation algorithms. Dehmeshki et al. [192] proposed an adaptive shericity-oriented contrast-based RG on the fuzzy connectivity map computed from the results of local adaptive thresholding segmentation. Diciotti et al. [193] presented a RG method with a fusion-segregation criteria using geodesic distances. Finally, Kubota et al. [174, 194] proposed a RG on an Euclidean distance map that are adjusted to handle juxtaposed structure more effectively.

d. Deformable model (DM) represents a class of segmentation methods based on iterative evolution of contour curves that models the boundary of target object, such as classic energy minimization-based active contour (AC) [223], edge-based geodesic AC [224], and region-based variational level-set (LS) [50]. One of the earliest work on volumetric lung nodule segmentation reported in literature was by Kawata et al. $[195,196]$ which adopted the geodesic AC approach by [225]. El Baz and Farag et al. [197, 198] have adopted the energy minimization approach by Kass et al. [223] with a prior appearance model by Markov random field (MRF) and a current appearance model by a bi-modal linear combination of discrete Gaussians. Farag et al. [201] proposed a variational LS solution with adaptive prior probability term for nodule segmentation. Yoo et al. [200] adopted the multiphase LS framework by Vese and Chan [226] to present an asymmetric 3-phase LS segmentation method for part-solid nodules. These approaches are adopted to evolve 3D surface boundary directly. In Way et al. [199], an approach to derive volumetric segmentation by 2D ACs applied to successive axial slices with 3D gradient, 3D curvature and mask energy terms in order to facilitate continuity along slice depths.

e. Dynamic programming (DP) here refers to a variational energy minimization approach for detecting optimal contours in images [227]. It guarantees to find non- 
iteratively the energy's global minimum among all possible contours, assuring its optimality $[227,228]$. This global optimality is an attractive property of this approach leading to better reproducibility. DP has been successfully applied to detection, tracking, and matching the boundary of general objects in 2D images [228]. Xu et al. [202] also applied this to 2D nodule boundary detection with lesion contour discontinuity detection by transforming an image from the Cartesian to polar coordinate system. An inherent issue to this approach is that its generalization to higher dimensional space is not straightforward. Several methods to extend this $2 \mathrm{D}$ approach to $3 \mathrm{D}$ surface detection for volumetric nodule segmentation have been reported. In Wang et al. [204], a sequence of 2D DPs are applied to successive slices with constraints for lesion center and radius from neighboring slices along the third dimension. This is repeated to the three orthogonal directions and the results are then fused. Wang et al. [203] proposed a method to transform a 3D VOI to a 2D image by transforming the $3 \mathrm{D}$ spherical to the $2 \mathrm{D}$ polar coordinate system along the points on the unit-sphere sampled in the order of a spiral from north to south pole. After this spiral scanning transformation, the standard 2D DP was applied to detect 3D lesion boundary.

f. Spherical/ellipsoidal model fitting exploits the proximity of CT lung nodule appearance to the standard Gaussian intensity model. Such an approximation model with isotropic Gaussian has been used in an early work for computer-aided detection of nodules [93]. For segmentation, both ellipsoidal (anisotropic Gaussian) and spherical (Laplacian of Gaussian) models have been exploited to approximately segment and estimate the size of nodule lesions. Okada et al. [205-207] proposed a robust estimation method for fitting the anisotropic Gaussian intensity model (RAGF: robust anisotropic Gaussian fitting) by posing the problem as the scale selection over an anisotropic scale-space [206]. At each scale, the Gaussian model is fit to a nodule image by using the mean shift algorithm [229]. Then the most stable scale that minimizes Jensen-Shannon divergence [230] computed over the varying scales determines the final outcome. In Diciotti et al. [209], the nodule size was estimated by using the multi-scale Laplacian of Gaussian (LoG) fil- 
tering [231]. The characteristic scale defined over the LoG scale-space was adopted as the lesion's size estimate and as an initialization of their RG-based segmentation method [193]. Jirapatnakul et al. [208] also studied this method as their nodule size measurement.

g. Probabilistic classification (PC) is another popular approach where each voxel is probabilistically classified as the nodule or the other structures. Probability distributions such as class-conditional likelihoods and prior distributions for each class must first be estimated from data. At each voxel, the classification decision is then casted as the standard estimation framework, such as maximum a posteriori (MAP), maximum likelihood (ML), and likelihood ratio test (LRT) [113]. Zhang et al. [119,210] proposed a MAP approach by using the MRF as the prior and Gaussian mixture model (GMM) as the class-conditional model estimated by offline training [119] or online for each image [210]. Okada et al. [211] proposed an approach based on LRT where foreground and background likelihoods were estimated online over a joint spatio-intensity domain from the results of the RAGF [207]. In Zhou et al. [212, 213], likelihood distributions were estimated by nonparametric kernel density estimator (KDE) then Bhattacharya distance was used as their classification criterion. Browder et al. [214] also proposed a ML approach for three classes (solid nodule, nonsolid nodule, and parenchymal tissue), where a Gaussian model is used to define each distribution. In Tao et al. [215], likelihoods are derived by GMMs over a subspace found by linear discriminant analysis of various intensity features, yielding probability maps. Final segmentation is given by thresholding the map with a shape-prior.

h. Discriminative classification (DC) casts the segmentation problem as a voxelwise classification similar to PC, however the classifiers are built by using generic supervised machine learning (ML) algorithms without explicitly estimating probability distributions [113]. Numerous methods exist for supervised discriminative classifiers in the ML literature. For nodule segmentation, only a few approaches have been adopted. Van Ginneken [216] proposed a soft-segmentation method where a function is learned to map various input intensity-based features computed at a voxel to a probability of the voxel 
being a part of a nodule. The output probability values for the training set were collected from multiple ground-truth segmentations. The k-nearest neighbor regression was used to establish this function. Zinoveva et al. [217] proposed a similar soft segmentation method by using a decision tree classifier with CART algorithm [232].

i. Mean shift (MS) is a segmentation approach based on an iterative feature space analysis [229]. MS algorithm performs a clustering of feature data points by iteratively seeking from each data point a mode of non-parametric distributions estimated by KDE [229]. Unlike the standard gradient descent algorithm [113], MS is provably convergent without requiring to tune the learning parameter thus can be implemented efficiently. Several works have adopted MS for the purpose of lung nodule segmentation. Okada et al. [218] proposed a robust nodule segmentation method that applied MS in the 4D joint spatio-intensity domain to refine the RAGF results, characterizing a nodule by an anisotropic Gaussian. Nie et al. [219] proposed a MS-based 2D nodule segmentation method over a feature space that combines the convergence index to the $3 \mathrm{D}$ joint spatiointensity domain. Finally, RAGF method proposed by Okada et al. [205, 207] extended the MS algorithm to Gaussian scale-space [231] and applied it to estimate the covariance for robustly fitting a Gaussian to data.

j. Graph-cuts (GCs) and Watersheds (WSs) , GCs [233] and WSs [45] are the other well-known techniques of standard image segmentation that have been adopted to the nodule segmentation problem. Zheng et al. [220,221] applied GC to derive their initial 2D nodule segmentation in their coupled segmentation-registration method with B-spline nonrigid registration [234]. Goodman et al. [222] utilized WC in their volumetry study. Each nodule was first segmented by using WS semi-automatically then a model-based shape analysis was performed to determine anatomical characteristics of various nodule types.

These techniques have been adopted to a number of commercially available semiautomatic software package and put into the clinical practice today. Many reproducibility studies for lung nodule volumetry have investigated performance of such software pack- 
ages [235-240]. De Hoop et al. [241] compared six packages (Advantage ALA, GE, v7.4.63; Extended Brilliance Workspace, Philips, EBW v3.0; Lungcare I, Siemens, Somaris 5 VB 10A-W; Lungcare II, Siemens, Somaris 5 VE31H; OncoTreat, MEVIS v1.6; Vitrea, Vital images v3.8.1) and found that substantial variations in segmentation performance exist among current lung nodule software packages. A summary of the previously reported methods for lung nodule segmentation is given in Table 4.

TABLE 4: Studies on volumetric nodule segmentation reported from 1998 to 2011. Studies are ordered by their publication year. The purpose, type, and basic idea of each reported segmentation method are briefly described. Data and method used for validation of proposed methods are also described. CCL: connected component labeling; DP: dynamic programming; ECLAP: early lung cancer action program; EM: expectation-maximization; GGO: ground-glass opacity (non-solid and part-solid) nodules; GMM: Gaussian mixture model; GT: ground-truth; kNN: k-nearest neighbor; LDA: linear discriminant analysis; LS: level-sets; MAP: maximum a priori; MO: morphological operations; MRF: Markov random fields; RMS: root mean square; SMDC: selective marking and depth constrained;

\begin{tabular}{|c|c|c|c|}
\hline Study & Nodule Type(s) & Method(s) & Data and validation \\
\hline $\begin{array}{l}\text { Kawata et } \\
\text { al. } \quad[195, \\
196]\end{array}$ & $\begin{array}{l}\text { solitary, } \\
\text { solid }\end{array}$ & $\begin{array}{l}\text { - Deformable model: geometric } \\
\text { deformation flow of 3D LS sur- } \\
\text { face proposed by Caselles et } \\
\text { al. [225] }\end{array}$ & $\begin{array}{l}\text { - } 62 \text { nodules (47 malignant } 15 \text { be- } \\
\text { nign) between } 6 \text { to } 25 \mathrm{~mm} \\
\text { - Qualitative: correct segmenta- } \\
\text { tion of nodules with ill-defined } \\
\text { surface; malignancy classifica- } \\
\text { tion with two 3D surface charac- } \\
\text { teristics }\end{array}$ \\
\hline $\begin{array}{l}\text { Yankelevitz } \\
\text { et al. [167, } \\
184]\end{array}$ & $\begin{array}{l}\text { small, } \\
\text { solitary, } \\
\text { solid }\end{array}$ & $\begin{array}{l}\text { - Threshold-based (2D [184]/ 3D } \\
[167]) \\
\text { - k-means segmentation for auto- } \\
\text { matic threshold estimation }\end{array}$ & $\begin{array}{l}\text { - Phantom: }(3.20 \text { and } 3.96 \mathrm{~mm}) \text {; } \\
\text { in-vivo: } 13-15 \text { nodules in repeat } \\
\text { CTs } \\
\text { - RMS error in volume measure- } \\
\text { ment: } \pm 3 \% \text { (3D); volumetry: } \\
\text { effective measurement of malig- } \\
\text { nant growth of nodules as small } \\
\text { as } 5 \mathrm{~mm} \text { (2D) with doubling } \\
\text { time less than } 177 \text { days (3D) }\end{array}$ \\
\hline $\begin{array}{l}\text { Zhao et al. } \\
{[183,185]} \\
\text { Wiemker } \\
\text { \& } \\
\text { Zwartkruis } \\
{[186]}\end{array}$ & $\begin{array}{l}\text { small, } \\
\text { jux- } \\
\text { tavascu- } \\
\text { lar }\end{array}$ & $\begin{array}{l}\text { - Threshold-based (2D [183]/ } \\
\text { 3D [185]): multi-criterion } \\
\text { automatic threshold estimation } \\
\text { with average gradients along } \\
\text { lesion contour and with bound- } \\
\text { ary shape compactness, lesion } \\
\text { segmentation by CCL and } \\
\text { MOs; efficient average gradient } \\
\text { computation [186] }\end{array}$ & $\begin{array}{l}\text { - } 9 \text { nodules }(<10 \mathrm{~mm}) \text { with man- } \\
\text { ual GT }(2 \mathrm{D}) \\
\text { - Mean difference of } 0.97 \text { pixels } \\
\text { was not statistically significant: } \\
p=0.90 \text { (2D) }\end{array}$ \\
\hline & & & continued on the next page ... \\
\hline
\end{tabular}


TABLE 4 - continued from the previous page

\begin{tabular}{|c|c|c|c|}
\hline Study & Nodule Type(s) & Method(s) & Data and validation \\
\hline $\begin{array}{l}\text { Xu et al. } \\
{[202]}\end{array}$ & $\begin{array}{l}\text { juxtavascular, } \\
\text { juxta- } \\
\text { pleural, } \\
\text { calcifi- } \\
\text { cation }\end{array}$ & $\begin{array}{l}\text { - Dynamic programming: 2D } \\
\text { contour optimization by DP } \\
\text { - Calcification removal by EM } \\
\text { classification of air, soft and cal- } \\
\text { cified tissues } \\
\text { - Semi-automatic contour correc- } \\
\text { tion by observers }\end{array}$ & $\begin{array}{l}\text { - } 4 \text { nodules } \\
\text { - Qualitative discussion only }\end{array}$ \\
\hline $\begin{array}{l}\text { Fetita et al. } \\
{[98]}\end{array}$ & $\begin{array}{l}\text { juxtavascular, } \\
\text { juxta- } \\
\text { pleural }\end{array}$ & $\begin{array}{l}\text { - Automatic, mathematical mor- } \\
\text { phology: gray-level MO with } \\
\text { SMDC-connection cost } \\
\text { - juxtavascular cases by morpho- } \\
\text { logical dilation and juxtapleural } \\
\text { cases by global lung segmenta- } \\
\text { tion }\end{array}$ & $\begin{array}{l}\text { ca. } 300 \text { nodules with } 2-20 \mathrm{~mm} \\
\text { diameters of } 10 \text { patients } \\
\text { - Detection performance: } 98 \% \\
\text { sensitivity and } 97 \% \text { specificity } \\
\text { for isolated and juxtavascular } \\
\text { nodules; } 90 \% \text { sensitivity and } \\
87 \% \text { specificity for juxtapleural } \\
\text { nodules }\end{array}$ \\
\hline $\begin{array}{l}\text { Ko et al. } \\
{[168]}\end{array}$ & $\begin{array}{l}\text { small, } \\
\text { solid/GGO }\end{array}$ & $\begin{array}{l}\text { - Threshold-based: two-value } \\
\text { thresholding with partial vol- } \\
\text { ume correction based on CT } \\
\text { intensity values }\end{array}$ & $\begin{array}{l}\text { - Phantom: } 40 \text { synthetic nodules } \\
\text { (<5 mm, } 20 \text { solid and } 20 \mathrm{GGO}) \\
\text { - Average error in volume mea- } \\
\text { surement: } 2.1 \mathrm{~mm}^{3}\end{array}$ \\
\hline $\begin{array}{l}\text { Kostis et al. } \\
{[95,188]}\end{array}$ & $\begin{array}{l}\text { small, } \\
\text { juxta- } \\
\text { pleural, } \\
\text { jux- } \\
\text { tavascu- } \\
\text { lar }\end{array}$ & $\begin{array}{l}\text { Mathematical morphology: } \\
\text { isotropic resampling for partial } \\
\text { volume effect; binary segmen- } \\
\text { tation by thresholding and CCL } \\
\text { followed by vascular subtraction } \\
\text { and pleural surface removal } \\
\text { with iterative MOs }\end{array}$ & $\begin{array}{l}\text { - } 105 \text { small nodules }(<10 \mathrm{~mm}) \text { of } \\
\text { two time-points } \\
\text { - Success rate: } 80 \% \text { for } 21 \text { jux- } \\
\text { tavascular cases; reproducibility } \\
\text { study in measuring the percent- } \\
\text { age volume changes [188] }\end{array}$ \\
\hline $\begin{array}{l}\text { Okada et } \\
\text { al. [205- } \\
207,218]\end{array}$ & $\begin{array}{l}\text { small, } \\
\text { jux- } \\
\text { tavas- } \\
\text { cular, } \\
\text { GGO }\end{array}$ & $\begin{array}{l}\text { Robust anisotropic Gaussian } \\
\text { fitting \& mean shift: robust } \\
\text { anisotropic Gaussian intensity } \\
\text { model fitting with mean shift } \\
\text { segmentation in 4D spatio- } \\
\text { intensity domain }\end{array}$ & $\begin{array}{l}\text { - } 77 \text { nodules of } 3-25 \mathrm{~mm} \text { diame- } \\
\text { ters of } 14 \text { patients } \\
\text { - Success rate: } 89.6 \% \text {; consis- } \\
\text { tency: } 1.12 \text { voxel mean error for } \\
\text { lesion center estimate when per- } \\
\text { turbing initialization }\end{array}$ \\
\hline $\begin{array}{l}\text { Kuhnigk } \\
\text { et al. [189, } \\
\text { 190] }\end{array}$ & $\begin{array}{l}\text { small, } \\
\text { jux- } \\
\text { tavas- } \\
\text { cular, } \\
\text { juxta- } \\
\text { pleural }\end{array}$ & $\begin{array}{l}\text { - Automatic, region growing \& } \\
\text { mathematical morphology: re- } \\
\text { gion growing and CCL for initial } \\
\text { segmentation } \\
\text { - Juxtapleural and juxtavascular } \\
\text { cases by convex hull and MOs } \\
\text { - Volume estimation with partial } \\
\text { volume effect handling }\end{array}$ & $\begin{array}{l}\text { - Phantom: } 31 \text { nodules of various } \\
\text { types; In-vivo: } 105 \text { nodules with } \\
\text { diameter larger than } 4.6 \mathrm{~mm} \text { of } \\
16 \text { patients } \\
\text { - Success rate: } 91.4 \% \text {; inter- } \\
\text { observer variability: } 0.1 \% \text { me- } \\
\text { dian error and } 7.1 \% \text { error at } \\
95 \% \text { limit; inter-scan variability: } \\
4.7 \% \text { median error and } 26.9 \% \\
\text { error at } 95 \% \text { limit; volumetry } \\
\text { median error with phantom: - } \\
3.1 \% \text { for vascularized cases; - } \\
10.2 \% \text { for juxtapleural cases }\end{array}$ \\
\hline & & & continued on the next page .... \\
\hline
\end{tabular}


TABLE 4 - continued from the previous page

\begin{tabular}{|c|c|c|c|}
\hline Study & Nodule Type(s) & Method(s) & Data and validation \\
\hline $\begin{array}{l}\text { Mullally et } \\
\text { al. [187] }\end{array}$ & $\begin{array}{l}\text { solitary, } \\
\text { solid }\end{array}$ & $\begin{array}{l}\text { - Automatic, threshold-basad: au- } \\
\text { tomating the selection of VOI } \\
\text { for thresholding-based segmen- } \\
\text { tation methods by Zhao et al. } \\
{[183,185] \text { \& Ko et al. [168] }}\end{array}$ & $\begin{array}{l}\text { - Phantom: } 40 \text { nodules (2.4 and } \\
4.9 \mathrm{~mm} \text { ); in-vivo: } 29 \text { nodules in } \\
\text { repeat CTs; manual GTs by a ra- } \\
\text { diologist } \\
\text { - Volume accuracy: } 43 \% \text { error for } \\
\text { phantoms; } 50 \% \text { error for in vivo } \\
\text { nodules }\end{array}$ \\
\hline $\begin{array}{l}\text { Shen et al. } \\
{[242]}\end{array}$ & juxtapleural & $\begin{array}{l}\text { - Surface analysis: lung surface } \\
\text { removal for juxtapleural nodule } \\
\text { segmentation by local surface } \\
\text { smoothing }\end{array}$ & $\begin{array}{l}\text { - } 20 \text { juxtapleural nodules of a pa- } \\
\text { tient } \\
\text { - Average RMS deviation from } \\
\text { median by various click points: } \\
<2 \% \text { except for one case } \\
\text { - Volumetry consistency: } 60 \% \text { of } \\
\text { all varying click points leads to } \\
\text { the same volume measure }\end{array}$ \\
\hline $\begin{array}{l}\text { Zhang et } \\
\text { al. [119, } \\
210]\end{array}$ & $\begin{array}{l}\text { GGO, } \\
\text { jux- } \\
\text { tavascu- } \\
\text { lar }\end{array}$ & $\begin{array}{l}\text { - Probabilistic classification: } \\
\text { MAP segmentation with a } \\
\text { conditional distribution by a } \\
\text { two-class GMM and with a prior } \\
\text { by MRF } \\
\text { - MAP optimization solved by it- } \\
\text { erated conditional modes } \\
\text { - juxtavascular cases by vessel } \\
\text { segmentation } \\
\text { - conditional distribution adapted } \\
\text { to each nodule to account for in- } \\
\text { tensity offsets [210] }\end{array}$ & $\begin{array}{l}\text { - } 23 \text { GGO nodules of } 8 \text { patients; } \\
\text { manual GTs by two radiologists } \\
{[210]} \\
\text { - Success rate: } 91.3 \% \text {; consis- } \\
\text { tency with } 3 \text { different clicks: } \\
0.96 \pm 0.02 \text { overlaps for all } 21 \\
\text { successfully segmented cases } \\
\text { - Average overlap with GTs: } \\
0.69 \pm 0.05 \\
\text { - Inter-observer consistency: } \\
0.73 \pm 0.04\end{array}$ \\
\hline $\begin{array}{l}\text { Okada et } \\
\text { al. } \quad[211] \\
\text { Okada et } \\
\text { al. } \quad[191, \\
243]\end{array}$ & $\begin{array}{l}\text { small, } \\
\text { jux- } \\
\text { tavas- } \\
\text { cular, } \\
\text { GGO, } \\
\text { juxta- } \\
\text { pleural }\end{array}$ & $\begin{array}{l}\text { - Probabilistic classification [211] } \\
\text { \& mathematical morphol- } \\
\text { ogy [191,243] } \\
\text { - likelihood ratio test in spatio- } \\
\text { intensity joint domain after ro- } \\
\text { bust anisotropic Gaussian fitting } \\
\text { by [207] } \\
\text { - juxtapleural cases by morpho- } \\
\text { logical opening and by prior- } \\
\text { constrained mean shift for rib } \\
\text { bone suppression }\end{array}$ & $\begin{array}{l}\text { - } 1312 \text { nodules of } 39 \text { patients; } 123 \\
\text { true-negative cases included } 108 \\
\text { juxtapleural cases } \\
\text { - Success rate: } 83.5 \% \quad \text { [211]; } \\
94.8 \% \text { [191,243] overall; } \\
71.5 \% \text { for the juxtapleural/true- } \\
\text { negative cases. }\end{array}$ \\
\hline $\begin{array}{l}\text { El-Baz et } \\
\text { al. [197] } \\
\text { Farag et } \\
\text { al. [198] }\end{array}$ & $\begin{array}{l}\text { general, } \\
\text { cavity }\end{array}$ & $\begin{array}{l}\text { - Deformable model: lesion } \\
\text { boundary optimization by fitting } \\
\text { a prior model with MRF and } \\
\text { an appearance model with a } \\
\text { bi-modal linear combination of } \\
\text { discrete Gaussians }\end{array}$ & $\begin{array}{l}\text { - } 350 \text { nodules with } 3 \text { to } 30 \mathrm{~mm} \text { of } \\
29 \text { patients (manual GTs by a ra- } \\
\text { diologist) } \\
\text { - Segmentation error: } \min 0.4 \% \text {, } \\
\text { max } 2.25 \% \text {, mean } 0.96 \%\end{array}$ \\
\hline
\end{tabular}


TABLE 4 - continued from the previous page

\begin{tabular}{|c|c|c|c|}
\hline Study & Nodule Type(s) & Method(s) & Data and validation \\
\hline $\begin{array}{l}\text { van Gin- } \\
\text { neken } \\
{[216]}\end{array}$ & general & $\begin{array}{l}\text { Discriminative classification: } \\
\text { soft segmentation by supervised } \\
\text { classifier } \\
\text { - kNN regression of voxel-wise } \\
\text { nodule probability with inten- } \\
\text { sity features (gradient magni- } \\
\text { tude, Hessian eigenvalues etc } \\
\text { over Gaussian scale-space) }\end{array}$ & $\begin{array}{l}\text { - LIDC1 dataset: } 23 \text { nodules with } \\
\text { manual GTs } \\
\text { - Average soft-overlap: } \\
0.66 \pm 0.18(0.52 \pm 0.25 \text { by [95]) } \\
\text { - Average percentage volume } \\
\text { error: } 23.7 \% \pm 124.5 \% \text { (by } \\
510.8 \% \pm 1577.7 \% \text { [95]). }\end{array}$ \\
\hline $\begin{array}{l}\text { Way et al. } \\
{[199,244]}\end{array}$ & general & $\begin{array}{l}\text { - Deformable model: successive } \\
\text { 2D active contour with 3D gra- } \\
\text { dient, 3D curvature and mask } \\
\text { energy terms with greedy opti- } \\
\text { mization }\end{array}$ & $\begin{array}{l}\text { - LIDC1 dataset: } 23 \text { nodules with } \\
\text { manual GTs } \\
\text { - Average overlap: ranging be- } \\
\text { tween } 0.07 \text { to } 0.63 \text { across vary- } \\
\text { ing probabilistic GTs } \\
\text { - Median percentage volume er- } \\
\text { ror: ca } 40 \%\end{array}$ \\
\hline $\begin{array}{l}\text { Goodman } \\
\text { et al. }[222]\end{array}$ & juxtavascular & $\begin{array}{l}\text { Watersheds segmentation fol- } \\
\text { lowed by a model-based shape } \\
\text { analysis to handle juxtapositions }\end{array}$ & $\begin{array}{l}\text { - } 50 \text { nodules of } 25 \text { patients } \\
(<20 \mathrm{~mm}) \text { with } 17 \text { irregu- } \\
\text { lar/spiculated margins, } 16 \\
\text { juxtapleural, 10 juxtavascular, } \\
\text { and } 2 \text { GGO cases } \\
\text { - Success rate: } 97 \% \text { over } 450 \\
\text { measures (3 time-points by } 3 \text { ob- } \\
\text { servers) }\end{array}$ \\
\hline $\begin{array}{l}\text { Zhou et al. } \\
{[212,213]}\end{array}$ & $\begin{array}{l}\text { GGO, } \\
\text { jux- } \\
\text { tavascu- } \\
\text { lar }\end{array}$ & $\begin{array}{l}\text { - Probabilistic classification: } \\
\text { voxel-wise classification by } \\
\text { comparing a non-parametric } \\
\text { kernel density estimate of GGO } \\
\text { intensity model with that of } \\
\text { each local neighborhood by } \\
\text { Bhattacharya distance } \\
\text { - Juxtavascular cases by eigen } \\
\text { analysis of Hessian }\end{array}$ & 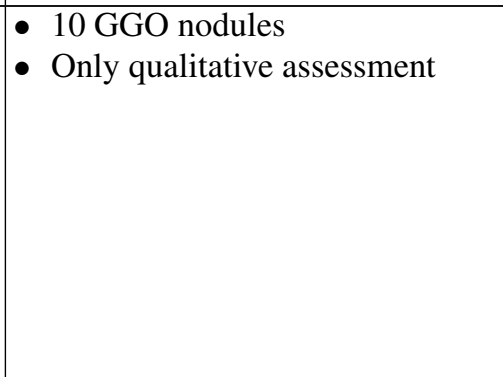 \\
\hline $\begin{array}{l}\text { Yoo et al. } \\
{[200]}\end{array}$ & $\begin{array}{l}\text { GGO, } \\
\text { jux- } \\
\text { tavascu- } \\
\text { lar }\end{array}$ & $\begin{array}{l}\text { - 3D deformable model: asym- } \\
\text { metric 3-phase deformable } \\
\text { model of two level-set functions }\end{array}$ & 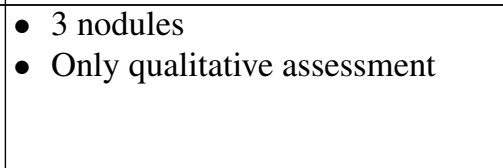 \\
\hline $\begin{array}{l}\text { Wang et al. } \\
{[203]}\end{array}$ & general & $\begin{array}{l}\text { - 3D dynamic programming: } \\
\text { transformation of 3D image to } \\
\text { 2D polar-coordinate image by } \\
\text { spiral scanning followed by 2D } \\
\text { contour optimization by DP }\end{array}$ & $\begin{array}{l}\text { - LIDC1 dataset: } 23 \text { nodules with } \\
4.0 \text { to } 33.6 \mathrm{~mm} \text { diameter; LIDC2 } \\
\text { dataset: } 73 \text { nodules with } 3.8 \text { to } \\
30.2 \mathrm{~mm} \text { diameter } \\
\text { - Average overlap (LIDC1): } 0.66 \\
\text { in [0.47,0.89]; average overlap } \\
\text { (LIDC2): } 0.64 \text { in }[0.39,0.87]\end{array}$ \\
\hline $\begin{array}{l}\text { Nie et al. } \\
\text { [219] }\end{array}$ & general & $\begin{array}{l}\text { - 2D mean shift clustering on a } \\
\text { feature domain of convergence } \\
\text { index by [245] }\end{array}$ & $\begin{array}{l}\text { - } 39 \text { nodules with manual GTs } \\
\text { - Average overlap: } 0.83\end{array}$ \\
\hline & & & continued on the next page \\
\hline
\end{tabular}


TABLE 4 - continued from the previous page

\begin{tabular}{|c|c|c|c|}
\hline Study & Nodule Type(s) & Method(s) & Data and validation \\
\hline $\begin{array}{l}\text { Zheng et } \\
\text { al. }[220] \\
\text { Zheng et } \\
\text { al. [221] }\end{array}$ & general & $\begin{array}{l}\text { - Graph-cut: 2D graph-cut seg- } \\
\text { mentation coupled with B-spline } \\
\text { non-rigid lung registration [220] } \\
\text { - Spatially coherent segmentation } \\
\text { by solving MRF with graph- } \\
\text { cut [221] }\end{array}$ & $\begin{array}{l}\text { - } 12 \text { nodules with manual GTs } \\
\text { - Mean percentage of the nodule } \\
\text { volume variation: } 0.8 \pm 0.6 \text { in } \\
\text { [220] }\end{array}$ \\
\hline $\begin{array}{l}\text { Browder et } \\
\text { al. }[214]\end{array}$ & $\begin{array}{l}\text { GGO, } \\
\text { small, } \\
\text { jux- } \\
\text { tavascu- } \\
\text { lar }\end{array}$ & $\begin{array}{l}\text { - Probabilistic classification: } \\
\text { 3-class (solid, non-solid, } \\
\text { parenchyma) voxel-wise prob- } \\
\text { abilistic classification with } \\
\text { Gaussian intensity model } \\
\text { - Bilateral filter by Tomasi used } \\
\text { for noise removal } \\
\text { - Juxtavascular cases by vessel re- } \\
\text { moval filtering }\end{array}$ & $\begin{array}{l}\text { - ELCAP dataset: } 75 \text { cases with } \\
5.6-17.5 \mathrm{~mm} \text { in diameter; man- } \\
\text { ual GTS by radiologists } \\
\text { - Median growth consistency by } \\
\text { geometric closeness metric: } \\
1.87 \text { ( } 3.12 \text { by radiologists) }\end{array}$ \\
\hline $\begin{array}{l}\text { Dehmeshki } \\
\text { et al. [192] }\end{array}$ & $\begin{array}{l}\text { juxtavascular, } \\
\text { juxta- } \\
\text { pleural }\end{array}$ & $\begin{array}{l}\text { - Region growing: sphericity- } \\
\text { oriented contrast-based region } \\
\text { growing from an optimum seed } \\
\text { point within a fuzzy connectiv- } \\
\text { ity map }\end{array}$ & $\begin{array}{l}\text { - } 815 \text { nodules with } 5-30 \mathrm{~mm} \text { in di- } \\
\text { ameter, } 98 \text { juxtapleural or jux- } \\
\text { tavascular cases } \\
\text { - Success rate: } 85-83 \%\end{array}$ \\
\hline $\begin{array}{l}\text { Diciotti et } \\
\text { al. [193] }\end{array}$ & $\begin{array}{l}\text { small, } \\
\text { jux- } \\
\text { tavascu- } \\
\text { lar, }\end{array}$ & $\begin{array}{l}\text { - Semi-automatic, region growing } \\
\text { - Target detection by Laplacian of } \\
\text { Gaussian filtering followed by } \\
\text { user selection } \\
\text { - 3D region growing segmenta- } \\
\text { tion using a fusion-segregation } \\
\text { criteria with geodesic distance }\end{array}$ & $\begin{array}{l}\text { - Phantom: } 60 \text { solid, juxtavascu- } \\
\text { lar, non-solid cases with 5.3- } \\
11 \mathrm{~mm} \text { in diameter; ITALUNG } \\
\text { dataset: } 98 \text { nodules; LIDC1 } \\
\text { dataset: } 23 \text { nodules } \\
\text { - Success rate: } \\
\text { (ITALUNG: } 79.7 \% \text { for jux- } \\
\text { tavascular); } 83.3 \% \text { (LIDC1: } \\
75 \% \text { for juxtavascular); volume- } \\
\text { try RMS error: } 1.0-6.6 \%\end{array}$ \\
\hline $\begin{array}{l}\text { Kubota et } \\
\text { al. [174, } \\
194]\end{array}$ & $\begin{array}{l}\text { small, } \\
\text { juxta- } \\
\text { pleural, } \\
\text { jux- } \\
\text { tavas- } \\
\text { cular, } \\
\text { solid, } \\
\text { GGO }\end{array}$ & $\begin{array}{l}\text { - Region growing: segmentation } \\
\text { and nodule extraction by re- } \\
\text { gion growing followed by con- } \\
\text { vex hull } \\
\text { - Nodule enhancement by coupled } \\
\text { competition-diffusion filtering } \\
\text { - Nodule core estimated as the } \\
\text { maximum component of Eu- } \\
\text { clidean distance map } \\
\text { - juxtapleural cases by estimating } \\
\text { region core with centricity map }\end{array}$ & $\begin{array}{l}\text { - LIDC1: } 23 \text { nodules; LIDC2: } 82 \\
\text { nodules; } 820 \text { nodules with man- } \\
\text { ual diameter GTs } \\
\text { - Average overlap (LIDC1): } \\
0.69 \pm 0.18(0.67 \pm 0.22 \text { by [190], } \\
0.57 \pm 0.20 \text { by [95], } 0.52 \pm 0.25 \\
\text { by [211]); average over- } \\
\text { lap (LIDC2): } 0.59 \pm 0.19 \\
(0.56 \pm 0.18 \text { by [190], } 0.45 \pm 0.21 \\
\text { by [211]) }\end{array}$ \\
\hline $\begin{array}{l}\text { Zheng et } \\
\text { al. [246] }\end{array}$ & GGO & $\begin{array}{l}\text { - 2D opacity map estimation: } \\
\text { thresholding opacity map esti- } \\
\text { mated by solving a linear equa- } \\
\text { tions system constructed with } \\
\text { the graph Laplacian }\end{array}$ & $\begin{array}{l}\text { - } 40 \text { slices of } 11 \text { patients; manual } \\
\text { GTs } \\
\text { - average shortest distance along } \\
\text { contours: } 4.2 \pm 4.9 \text { pixels }\end{array}$ \\
\hline \multicolumn{4}{|c|}{ continued on the next page ... } \\
\hline
\end{tabular}


TABLE 4 - continued from the previous page

\begin{tabular}{|c|c|c|c|}
\hline Study & Nodule Type(s) & Method(s) & Data and validation \\
\hline $\begin{array}{l}\text { Wang et al. } \\
\text { [204] }\end{array}$ & general & $\begin{array}{l}\text { - 3D dynamic programming: } \\
\text { multi-direction segmentation } \\
\text { fusion by sequential dynamic } \\
\text { 2D contouring ( }[202,203]) \\
\text { applied to three orthogonal } \\
\text { directions of a volume }\end{array}$ & $\begin{array}{l}\text { - LIDC1: } 23 \text { nodules for training } \\
\text { and LIDC2: } 64 \text { nodules for test- } \\
\text { ing } \\
\text { - LIDC1: Mean overlap: } 0.66 \text {, } \\
\text { true-positive rate (TPR): } 75 \% \text {, } \\
\text { false-positive rate (FPR): } 15 \% \text {; } \\
\text { LIDC2: Mean overlap } 0.58 \text {, } \\
\text { true-positive rate (TPR): } 71 \% \text {, } \\
\text { false-positive rate (FPR): } 22 \% \text {. }\end{array}$ \\
\hline $\begin{array}{l}\text { Tao et al. } \\
{[215]}\end{array}$ & GGO & $\begin{array}{l}\text { - } \text { Probabilistic classification } \\
\text { - GGO nodule class-conditional } \\
\text { probability map derived by an it- } \\
\text { erative LDA with GMMs of var- } \\
\text { ious intensity features } \\
\text { - Nodule segmentation by apply- } \\
\text { ing shape-prior probability mask }\end{array}$ & $\begin{array}{l}\text { - } 1100 \text { nodules with } 100 \text { GGO } \\
\text { nodules; } 60 \text { cases with manual } \\
\text { GTs } \\
\text { - Average overlap: } 0.68 \text {; voxel- } \\
\text { wise classification success rate: } \\
92.28 \% \text { overall; } 89.87 \% \text { GGO }\end{array}$ \\
\hline $\begin{array}{l}\text { Farag et al. } \\
\text { [201] }\end{array}$ & juxtapleural & $\begin{array}{l}\text { - 3D Deformable model: vari- } \\
\text { ational level-set segmentation } \\
\text { with narrow band implementa- } \\
\text { tion. }\end{array}$ & $\begin{array}{l}\text { - ELCAP database: } 397 \text { nodules } \\
\text { of } 50 \text { patients, } 115 \text { juxtapleural } \\
\text { cases } \\
\text { - Success rate: } 70 \% \text { for juxtapleu- } \\
\text { ral cases }\end{array}$ \\
\hline $\begin{array}{l}\text { Zinoveva } \\
\text { et al. [217] }\end{array}$ & general & $\begin{array}{l}\text { - Discriminative classification } \\
\text { - Soft segmentation } \\
\text { - CART decision tree classifier } \\
\text { trained with texture and inten- } \\
\text { sity features } \\
\text { - VI trimming post-processing. }\end{array}$ & $\begin{array}{l}\text { - LIDC2 dataset: } 39 \text { nodules with } \\
\text { 3-30 mm in diameter; manual } \\
\text { GTs by } 4 \text { radiologists } \\
\text { - Median soft-overlap: } 0.49 \text { and } \\
\text { 0.52 with VI trimming }\end{array}$ \\
\hline $\begin{array}{l}\text { Jirapatnaku } \\
\text { et al. }[247]\end{array}$ & juxtapleural & $\begin{array}{l}\text { - Surface analysis: robust esti- } \\
\text { mation of pleural surface, sur- } \\
\text { face removal by change detec- } \\
\text { tion over the estimated surface. }\end{array}$ & $\begin{array}{l}\text { - } 150 \text { solid juxtapleural nodules } \\
\text { - Success rate: } 98.0 \% \text { (81.3\% by } \\
\text { [248]) }\end{array}$ \\
\hline $\begin{array}{l}\text { Diciotti et } \\
\text { al. [249] }\end{array}$ & juxtavascular & $\begin{array}{l}\text { Shape analysis: refine an ini- } \\
\text { tial rough segmentation based } \\
\text { on a local shape analysis on 3-D } \\
\text { geodesic distance map represen- } \\
\text { tations }\end{array}$ & $\begin{array}{l}\text { - ITALUNG dataset: } 256 \text { small } \\
\text { nodules; LIDC12 datasets: } 157 \\
\text { small nodules } \\
\text { - Success rate: } 84.8 \% \\
\text { (ITALUNG) and } 88.5 \% \\
\text { (LIDC12) for automatic; } \\
\text { 91.0\% (ITALUNG) and 91.7\% } \\
\text { (LIDC12) for interactive mode. }\end{array}$ \\
\hline
\end{tabular}

\section{PET Segmentation Techniques}

Positron emission tomography (PET) with the glucose analog, 18F-2fluoro-2-deoxyD-glucose (FDG), has been widely used in oncology applications such as lung cancer detection and nodule segmentation. Using CT alone, target volume delineation of lung cancer is prone to inter-observer variability, with variations in the gross tumor volume (GTV) def- 
inition being as high as $700 \%$ in lung tissue [250]. However, incorporating PET enhances the result of tumor outlining, diagnostic evaluation of pulmonary nodules, and staging the mediastinum. The widely used quantifier in PET imaging is the standardized uptake value (SUV) that estimates the intensity of the lesion on PET. The SUV is calculated either pixelwise or over a region of interest (ROI) for each image at time $t$, as the ratio of tissue radioactivity concentration, $c(t)$, and injected dose at the time of injection divided by body weight:

$$
S U V=\frac{c(t)}{\text { injected dose }\left(t_{0}\right) / \text { body weight }}
$$

To define the tumor region, the most straightforward technique is to apply a thresholding based method. Automatic thresholding-based methods used the SUV parameter to estimate the optimal threshold that defines the tumor region. Paulino-et-al [251] used an SUV value of 2.5 to auto-contour the derived GTV. Other fixed thresholding-based methods define the tumor region by an arbitrary threshold value such as $40 \%, 42 \%$, or $50 \%$ of the maximum SUV [252-257]. In addition to the fixed thresholding-based techniques, there are other adaptive thresholding-based approaches that incorporate tumor volume, background activity, and source-to-background (S/B) ratios [258-263]. Nestle et al. [258] compared different GTVs obtained from different methods to look for the optimal threshold value. Four different GTV's are obtained using four different methods: (1) GTV vis obtained by visual interpretation; (2) $\mathrm{GTV}_{40}$ obtained by applying a threshold of $40 \%$ of the $\mathrm{SUV}_{\text {max }}$; (3) $\mathrm{GTV}_{2.5}$ obtained by applying a threshold equal to SUV $=2.5$; and (4) $\mathrm{GTV}_{b g}$ obtained by using phantom studies as the best fit obtained based on the tumor and background intensities. $\mathrm{GTV}_{\text {vis }}, \mathrm{GTV}_{2.5}$, and $\mathrm{GTV}_{b g}$ showed a strong correlation with the CT-derived GTV, whereas the $\mathrm{GTV}_{40}$ was shown to be unsuitable. Nestle et al. concluded that the variability of the differences was due to the inhomogeneity in the nodules appearance and the difference in their sizes.

The main limitations of thresholding-based techniques are that they are user- and system-dependent and do not consider some important factors in the tumor delineation such 
as target motion due to respiration and cardiac activity. In addition, a single threshold model lacks the incorporation of other factors such as tumor size and non-uniform distribution of FDG-activity [258]. In many cases, due to conditions such as necrosis and hypoxia in nonsmall cell lung cancer (NSCLC), a single threshold model cannot be obtained since these conditions create non-uniform uptake value. Experimental measurements of radioactive spheres in a phantom using thresholding-based methods show that the thresholding-based methods are unreliable in the clinical studies [258, 264-266].

To provide more reliable tumor delineation, statistical segmentation techniques cast the tumor segmentation within a statistical framework as an unsupervised classification problem. For a given data set composed of a set of items, a statistical classification framework attempts to label each item to with some level of certainty, like in [267]. For examples, FLAB [268] and 3-FLAB [269] are locally adaptive Bayesian segmentation approaches that are combined with a fuzzy measure. Each voxel will be assigned to its appropriate class based on its value and the values of its neighbors and also the noise model's parameters. In 3-FLAB which is an improvement of FLAB, three hard classes and three fuzzy transitions are incorporated and the model is evaluated on heterogeneous tumors as well as homogeneous ones. Based on unsupervised estimation and noise modeling, the Fuzzy C-Means clustering method (FCM) [270] and the Fuzzy Hidden Markov Chain (FHMC) [271] similarly attempt to find large groupings within the intensity distributions obtained from the PET image. The segmentation results of these fuzzy based methods show better tumor delineation with respect to the thresholding-based methods. However, they usually require an estimation of the initial class and they consider only the PET modality in their implementations.

More complex segmentation methodologies have been proposed to solve the lung tumor delineation problem [268, 271-279]. For example, Li et al. [278] used an adaptive region growing method that extracts the tumor boundaries using deformable models in PET. Avazpour et al. [280] used a region growing approach that is employed on co-registered 
PET/CT for exclusion of collapsed lung. Mohamed et al. [281] and Woods et al. [282] incorporate textural and structural features in their segmentation methods. To summarize the approaches presented for the segmentation of lung nodules from PET images, Table 5 briefly describes the number of the patients enrolled in each study and the type of the nodule delineation approach with respect to the methodology, the approach dimension, and the approach automation level.

TABLE 5: Summary of lung nodule segmentation approaches from PET images. For each study, the table summarizes the number of the patients enrolled in the study and the type of the nodule delineation approach with respect to the methodology, the approach dimension, and the automation level.

\begin{tabular}{|l|l|l|l|l|}
\hline \multicolumn{1}{|c|}{ Study } & Patients & Delineation Approach & Dim & AL \\
\hline Kiffer et al. [283] & 15 & Co-registration & 2D & A \\
\hline Munley et al. [284] & 35 & Manual registration & NA & NA \\
\hline Nestle et al. [256] & 34 & visual & NA & NA \\
\hline Mah et al. [252] & 30 & Thresholding & 3D & A \\
\hline Erdi et al. [255] & 11 & Thresholding & NA & A \\
\hline Bradley et al. [253] & 26 & Thresholding & 2D & A \\
\hline Deniaud et al. [257] & 101 & Visual & NA & NA \\
\hline $\begin{array}{l}\text { van Der Wel et } \\
\text { al. [285] }\end{array}$ & 21 & Visual & NA & NA \\
\hline $\begin{array}{l}\text { Ashamalla } \\
\text { al. [265] }\end{array}$ & 19 & Thresholding & NA & A \\
\hline $\begin{array}{l}\text { Hatt et al. [271] } \\
\text { Hatt et al. [268] }\end{array}$ & NA NA & $\begin{array}{l}\text { Fuzzy hidden markov } \\
\text { chain }\end{array}$ & NA & A \\
\hline $\begin{array}{l}\text { Avazpour } \\
\text { al. [280] }\end{array}$ & Fuzzy classification & 3D & A \\
\hline Hatt et al. [269] & Region growing & 2D & A \\
\hline $\begin{array}{l}\text { Dim } \\
\text { AL } \\
\text { NA }\end{array}$ & $\begin{array}{l}\text { Fuzzy classification } \\
\text { denotes the approach dimension (2D, 3D, or 4D), } \\
\text { denotes Non-Applicable. }\end{array}$ & NA & A \\
\hline
\end{tabular}

As PET acquisition takes several minutes, it is influenced by the patient's breathing and motion. These respiratory movements and cardiac actions result in the target motion which creates significant image blur that affects the accuracy of GTV estimation. On the other hand, using CT only implies a large uncertainty in the result of target volume delin- 
eation, specially in NSCLC $[286,287]$. Reported cases, in which the GTV delineated based on CT, include abnormalities that appear totally devoid of FDG activity and can safely be removed from the GTV. Thus, the combination of PET and CT information has been studied in order to improve the target volume definition especially in NSCL and cases with atelectasis. In this regard, the recent studies have shown that the integration of PET information in the treatment planning has significantly reduced the inter-observer contouring variability [286, 287].

To combine PET and CT information, a fusiontechnique should be applied to integrate the PET and CT images. The fusion techniques can be classified into one of three categories: (1) visual fusion in which both imaging modalities are simply considered side by side, (2) software fusion, and (3) hardware fusion. Using visual vision, Kiffer et al. [283] showed that by using PET information the outlined volume has changed in $26.7 \%$ of the cases. They conclude that the variability on the volume estimation is due to the detection of abnormal mediastinal nodes on PET which cannot be detected on CT. Nestle et al. [256] and Munley et al. [284] used software fusion techniques that reported a significant change in the target volume extraction when compared to CT-defined volume. Nestle et al. [256] has documented that in 6 out of 17 patients with dystelectasis or atelectasis, the size of the delineated target was reduced with a median change of 19.3\%. Munley et al. [284] reported an increase in the GTV in 34\% of the cases when compared to CT. Erdi et al. [255] performed a study on patients who received CT and PET scanning using the same device. GTV, PTV, and normal tissues were initially contoured on the CT and then CT and PET were registered in a treatment-planning system. There was an average increase of 19\% in the PTV volume in 7 out of 11 patients and an average decrease of $18 \%$ in the PTV in the other four patients. Van Der Wel et al. [285] showed that the GTV decreased significantly when shifting from the CT-only to the fused PET/CT in 21 patients, thus allowing dose escalation. Further studies on the rate of recurrence when PET is used showed that only 1 out of 44 patients developed the tumor recurrence [288]. Steenbakkers et al. [286] and 
Fox et al. [287] used software fusion methods and analyzed the observer variation in two phases, one with CT only and another one with fused PET/CT. The two studies addressed the issue of inter-observer variation reduction using matched PET and CT and concluded that the PET/CT software fusion is superior to visual fusion.

Table 6 summarizes the published studies on the effect of PET on GTV as a complementary to CT. For each study, the number of patients, the PET/CT fusion method, and the increase and decrease in the GTV as a percentage of the total number of the study cases are reported. These studies reported that the PET/CT fusion has improved the GTV estimation and thus is preferable for the treatment optimization in NSCLC. However, some well known technical issues such as the resolution of PET, the exact tumor edge definition, and the mis-registration between PET and CT images need further investigations.

TABLE 6: Assessing effect of PET/CT on GTV. For each study, the number of patients, the PET/CT fusion method, and the increase and decrease in the GTV as a percentage of the total number of the study cases is reported.

\begin{tabular}{|l|l|l|l|l|}
\hline \multicolumn{1}{|c|}{ Study } & Patients & $\begin{array}{l}\text { PET/CT Fusion } \\
\text { Method }\end{array}$ & $\begin{array}{l}\text { GTV in- } \\
\text { crease }\end{array}$ & $\begin{array}{l}\text { GTV de- } \\
\text { crease }\end{array}$ \\
\hline Kiffer et al. [283] & 15 & $\begin{array}{l}\text { Graphical Co- } \\
\text { registration }\end{array}$ & $27 \%$ & NA \\
\hline Munley et al. [284] & 35 & Visual & $34 \%$ & NA \\
\hline Nestle et al. [256] & 34 & $\begin{array}{l}\text { Visual (side-by- } \\
\text { side) }\end{array}$ & $9 \%$ & $26 \%$ \\
\hline Mah et al. [252] & 30 & Software & $22 \%$ & NA \\
\hline Erdi et al. [255] & 11 & Software & $64 \%$ & $36 \%$ \\
\hline Bradley et al. [253] & 26 & Software & $46 \%$ & $12 \%$ \\
\hline Deniaud et al. [257] & 101 & Software & $26 \%$ & $23 \%$ \\
\hline $\begin{array}{l}\text { Ashamalla et } \\
\text { al. [265] }\end{array}$ & 19 & Hardware & $26 \%$ & $26 \%$ \\
\hline $\begin{array}{l}\text { van Der Wel et } \\
\text { al. [285] }\end{array}$ & 21 & Visual & $14 \%$ & $52 \%$ \\
\hline $\begin{array}{l}\text { Avazpour } \\
\text { al. [280] }\end{array}$ & 11 & Software & NA & NA \\
\hline
\end{tabular}




\section{Nodule Segmentation Challenges}

Several challenges and aspects have been facing lung nodule segmentation techniques such as that the ability of a technique to segment the challenging types of nodule, the automation level of the technique and its robustness. This section will briefly address each of these challenges.

a. Nodule Types $\mathrm{CT}$ values for parenchymal tissues differ significantly from those for soft tissues. Therefore, segmentation of solitary and large solid nodules is technically straightforward. Problems arise when targeting 1) small nodules, 2) nodules attached to vessels (juxtavascular cases), 3) nodules attached to parenchymal wall and diaphragm (juxtapleural cases), and 4) ground-glass opacity nodules. The following outlines the nature of each issue and current approaches handling them.

Small nodules segmentation plays an important role for early detection of lung cancers [177]. The advent of high-resolution thin-slice CT has enabled to visualize small nodules with less than $5 \mathrm{~mm}$ in diameter which could not be made visible by previous generation CT technologies. Accurate segmentation of such small nodules is needed to assess malignancy of the lesions by measuring their growth rate as will be discussed in Section II.E.1. Partial volume effect (PVE) is the main technical concern when handling small nodules. Due to spatial discretization used for the CT imaging, a single voxel may represent more than one tissue type, resulting in averaging of their intensity values. This causes PVE, image blur especially at lesion boundaries, making their segmentation difficult. PVE becomes more pronounced when handling smaller lesions because the percentage of errors over the lesion volume would increase in such a case. This makes accurate area/volume measurement for small nodules more challenging. A number of approaches have been proposed to handle PVE in small nodule segmentation $[95,168,190,207]$. Ko et al. [168] presented partial-volume method (PVM) for estimating nodule volume based on the consistency of the average attenuation amounts. Their phantom study demonstrated that PVM yields higher accuracy in volumetry than various thresholding methods. Kuhnigk et 
al. [190] proposed segmentation-based partial volume analysis (SPVA) that extended the PVM approach to incorporate segmentation of VOI into nodule core, parenchyma area, and partial volume region. A histogram sampled from the partial volume region was used to estimate the nodule's volume near its boundary. Kostis et al. [95] proposed isotropic resampling of volumetric images to mitigate PVE and also presented an upper-bound for the PVE error of a perfect circle. Finally, RAGF proposed by Okada et al. [207] yields an ellipsoidal approximation of lesion boundary. When segmenting a small nodule, volume measure derived directly from a fitted ellipsoid may be more accurate than voxel-wise segmentation results due to PVE.

Lung nodules are frequently attached to other pulmonary structures such as airways, blood vessels, parenchymal walls, and diaphragm. Because the CT values of nodules and these non-target structures are often very similar, accurate delineations of the extent of nodules from these structures become a difficult technical challenge.

Juxtavascular nodules refer to nodules that are attached to blood vessels. There are many studies that have addressed a solution for handling such juxtavascular cases [95, $98,119,174,185,190,192,193,200,202,207,212-214,222,248,249]$. One common approach for this purpose is morphological filtering [95, 98, 119, 185, 190, 248]. Because the portion of nodules that attaches to vessels/airways is typically small with respect to the total extent of the 3D nodule surface, basic MOs, such as erosion, dilation, and opening, are often effective for most juxtavascular cases $[98,185]$. More complex morphological filtering based on iterative [95, 248] and successive [190] combinations of these basic operators, convex hull operations [174, 190], and 3D moment analysis [214] have also been adopted as a post segmentation refinement method. Geometric/shape-constrained segmentation is another popular approach in this context [192, 193, 207,222, 249]. This approach integrates shape-based prior information within the segmentation process in order to bias the results toward a spherical/nodular shape and suppress elongated non-target structures attached to the target. Gaussian model fitting [207], eigen analysis of the Hessian ma- 
trix [212,213], sphericity oriented region growing [192], geodesics distance constraints between connected-components [249], and a steepest-ascent test [174] are some examples of this type of geometric-constraint approaches.

Juxtapleural nodules refer tocases that are attached to parenchymal wall or diaphragm. A number of studies have addressed a solution for handling such juxtapleural cases [95, 98, 174, 190-192, 201, 202, 207, 242, 247, 248]. Pleural surface removal (PSR) is the most common approach $[95,98,190,191,242,247,248]$. PSR can be addressed either globally or locally. The global methods first segment the entire lung from a CT image then use the result as a negative mask to avoid the non-target wall regions to be included in the nodule segmentation results. Morphological filtering was the common approach similar to juxtavascular cases $[98,190,242]$. In order to accurately segment lung walls, juxtapleural nodules must be discounted. Local surface smoothing [242] and convex hull operation [190] have specifically been adopted for this purpose. The local PSR methods perform the same task of removing pleural surface within a VOI $[95,191,247,248]$. Morphological filtering is also a popular approach in this context [95, 191,248]. A local patch of pleural surface can be approximated as a 3D plane. Kostis et al. [95] used a morphological filtering with a disk-shape kernel and Reeves et al. [248] presented an iterative clipping plane adjustment, exploiting this planar assumption. Beyond the planar model, a polynomial surface can be fit to VOI to improve the accuracy [247]. Another approach is to robustly detect the center of juxtapleural nodules because many reported general-purpose methods fails to do this. Prior-constraint mean shift [191, 243], robust nodule core detection by centricity transformation [174], and maximum curvature point [201] are the examples of robust methods addressing this task.

Ground glass opacity (GGO) nodule refers to a type of nodules with subsolid CT values that are significantly lower than those of typical solid nodules. Based on whether any solid components are present or not, they are categorized into two types: non-solid/pure and part-solid/mixed. Segmentation of GGO nodules poses a technical challenge because 
it is hard to delineate their subtle boundaries and to model their irregular appearances. In clinical practice, the increased image resolution by the recent CT technologies have made it possible to study these small GGO nodules that were previously undetectable. Their growth are often very slow [180], however such GGO nodules, especially the mixed ones, have been found to have a high chance of being malignant [178]. Recent clinical studies have confirmed that they represent the histologic spectrum of peripheral adenocarcinomas, including the premaligannt atypical adenomatous hyperplasia (AAH) and the malignant bronchioloalveolar carcinoma (BAC) [179]. A small non-solid GGO representing $\mathrm{AAH}$ or $\mathrm{BAC}$ can slowly evolve into an invasive lung adenocarcinoma over the period of 10 years [180]. Due to the clinical interests and technical challenge, many attempts have recently been made to propose segmentation solutions for this nodule subtype $[119,168,174,194,200,210-215,218,246]$. Most common approach among them was the voxel-wise probabilistic classification in order to handle the subtle and irregular lesion appearances [119,210-215]. In this approach, segmentation is performed by assigning each voxel with a nodule/background label according to its probabilistic decision rule derived from training data. MAP segmentation with a MRF prior [119,210], LRT segmentation in the joint spatio-intensity domain [211], classification by Bhattacharya distance with a non-parametric KDE-based intensity likelihood [212,213], 3-class ML segmentation [214], and classification by Otsu thresholding with class-conditional probability map derived by an iterative linear discriminant analysis and shape-prior mask [215] are examples of various classification and modeling methods explored in the literature. Other interesting approaches for GGO nodule segmentation include RAGF approach [207], asymmetric 3-phase level-set segmentation [200], robust region growing [194], and graph Laplacian-based opacity map estimation [246].

Overall, above-cited studies have agreed that the juxtapleural and part-solid GGO nodules are the most difficult types of nodules to segment accurately. Developing typespecific and general nodule segmentation that can handle these difficult cases remains an 
unresolved challenge.

b. Automation In a computer-aided diagnosis (CAD) system, lung nodule segmentation serves as a sub-component of the overall system. Beyond the obvious accuracy requirement, the usability of the segmentation methods play a significant role in assessing effectiveness of the overall system. In this sense, to reduce labor burden of users is one of the critical goals of the segmentation methods since an accurate but labor-intensive method that requires a large amount of precise manual user-interactions would be less preferred. In this application context, previously proposed segmentation methods are classified into two types: automatic and semi-automatic.

The automatic approach takes a CTimage as an input then simultaneously segments all nodules present in the image without their locations specified by users $[98,187,212$, 213,215]. Early methods with gray-scale MM filtering by Fetita et al. [98] and with automatic locally adaptive thresholding by Mullally et al. [187] have addressed this simultaneous segmentation of all nodules in volume. More recently, the probabilistic approach, targeting GGO nodule segmentation, have been exploited to address a couple of automatic segmentation methods. Zhou et al. [212,213] used Bhattacharya distance-based classification with a GGO intensity distribution modeled by the non-parametric KDE. Tao et al. [215] employed a class-conditional probability map modeled by a GMM over a subspace of various intensity features, such as gray-level co-occurrence matrix (GLCM), local binary pattern (LBP), 3D Harr wavelet, derived by an iterative linear discriminant analysis. In both methods, the automation is realized by deriving a probabilistic model of general nodule appearance.

On the other hand, the semi-automatic approach assumes that the location of target nodules is known. In this assumption, a segmentation methods takes a VOI as an input and assumes that the VOI contains the entire extent of a single nodule. Many segmentation algorithms fall into this category since their iterative process requires initializations or seeds. The amount of seeds required ranges from a single user click with robust model fit- 
ting [207] and region growing [174, 192, 194, 207] to an entire 3D contour with deformable models [195-199,201]. Note that these semi-automatic methods can also be automated by using it together with a CADe nodule detection system which automatically provides the seeds to one of these semi-automatic segmentations.

Error correction is another important usability aspect of nodule segmentation systems. As described in the previous section, there are methods for refining/correcting segmentation results for specific types of nodules that are difficult to segment, exploiting the nature of the specific nodule types. In order to improve their usability, such error correction process can be automated with an automatic detection of nodule types or of segmentation failures. Such nodule type-specific automatic error correction has been presented for juxtavascular [249] and juxtapleaural cases [191]. Semi-automatic error correction is of interest in its clinical context. Such an approach can provide users stricter control on details of segmentation outcomes in order to better exploit the domain knowledge of expert observers during its clinical workflow. Some segmentation algorithms allow users to interactively constrain segmentation results by specifying certain voxels to be a part of the results. For example, the optimization process used in the DP algorithm can take an arbitrary number of voxels as its hard constraints such that they are fixed to be a part of the final lesion contour. Xu et al. [202] exploited this pixel-wise hard constraints in their semiautomatic segmentation refinement by letting users to specify correct contour voxels with mouth-clicks.

c. Robustness The semi-automatic procedure used in many segmentation methods involves user-determined seed points to indicate a target nodule to be segmented as described in the previous section. Different observers, or a single observer studying the same scan more than once, may produce different seed points, causing the intra- and interobserver variances with different segmentation results of the same nodule. As will be described in Section II.E.1, reduction of such variance plays a key role to realize repeatable and reproducible volumetry. For example, in percentage error of estimated volume, this 
inter-observer variance can be as high as $20 \%$ [170]. A number of robust approaches have been studied to design a reliable and robust segmentation solution against such variabilities $[174,190,192,194,207,247]$.

One of the common approaches in this context is to robustly estimate nodule's center/core from a user-specified seed in order to reduce the intra- and inter-observer variance of the semi-automatic methods. The result of this process can be treated as the optimal seed that refines the user-specified seed to be robust against the perturbations due to user interactions. RAGF proposed by Okada et al. [207] estimated a nodule center by majority-voting of convergences of mean shift procedures initialized by voxels randomly sampled around the initial seed. Kuhnigk et al. [190] detected the optimal seed as the convergence of an iterative local maximum search of 3D distance map around the initial seed. Dehmeshki et al. [192] chose the optimal seed as the voxel of the highest intensity value among the maximum distance voxels in a 3D distance map. Finally, in Kubota et al. [174, 194], the optimal seed was estimated by the voxels with maximum centricity values computed over a $3 \mathrm{D}$ distance map.

Some common robust estimation techniques have also been adopted to the nodule segmentation problem. A model fitting process can be made robust by ignoring outlier samples and only considering inlier samples. This standard principle has been applied in the RAGF method by fitting an anisotropic Gaussian only with samples within a basin of attraction defined by the mean shift [207], and in the robust lung surface modeling by fitting a polynomial surface to lung wall only with samples that lie on the pleural surface but not on the nodule [247]. A perturbation-based stability analysis was adopted in the RAGF method by Okada et al. [207] in order to determine the most stable scale for a Gaussian scale-space by minimizing the Jensen-Shannon divergence computed with a set of the Gaussians estimated with perturbed scales.

As discussed in Section II.D.4.a, variability among various types of nodules poses significant challenge for accurate nodule segmentation. Some robust approaches to han- 
dle these various nodule types have been proposed yielding general-purpose segmentation methods. The RAGF method [207] imposes the ellipsoidal constraints for handling juxtaposed cases. A similar approach with Laplacian of Gaussian filtering, imposing the spherical constraints of various sizes, has also been applied to detection and rough segmentation of nodules [208,209]. Recently, Kubota et al. [174, 194] have proposed a robust region growing method that successfully handles all types of nodules.

d. Validation Validation refers to experimental procedures to measure the accuracy of segmentation methods to be evaluated. It is important not only for choosing the best performing method for a particular application but also deriving some critical clinical parameters, such as the minimum size of measurable lesions and the shortest time duration for repeat CT in follow-up studies [170, 248]. Designing the effective validation procedure is a major challenge in the lung nodule segmentation research. This is because manual lesion contouring by observers used to prepare the ground-truth (GT) segmentations is labor-intensive, making it difficult to create large GT datasets. Another reason is the intraand inter-observer variability for nodule segmentation, indicating that even the manual GTs prepared by expert radiologists can vary significantly [289].

The most accurate gold standard for validation currently available is chest phantom scans, where an artificial device simulating nodules and other parenchymal structures of known sizes is imaged by a standard CT scanner. Many studies have utilized various phantom scans for tuning parameters and bench-marking their best possible performance in vivo [167, 168, 184, 187, 190, 193, 244, 290-293].

Experimental validation of nodule segmentation methods with actual patient scans poses difficulty as described above. One common approach is based on subjective judgment of segmentation results by visual inspection of expert observers. In this approach, the observer(s) classifies each case as success or failure, then the rate of successfully segmentation is reported [95, 119, 190, 192, 193, 201, 207, 218, 222, 247, 249].

Some authors prepare and use GT datasets of 2D segmentation [183] and of 3D 
segmentation for solid nodule $[174,187,197,220]$ and for GGO nodules [174, 210, 214, $215,246]$. With such GTs, various segmentation methods have been validated by a number of quantitative accuracy and error measures, such as 1) overlap ratio (a fraction of cardinality of the intersection and the union of voxel sets for a lesion's segmentation and its GT) [174, 199, 203, 204, 210, 215-217, 219], 2) percentage voxel error rate (percentage of voxels mis-segmented with respect to the total number of voxels in a nodule) $[197,199$, 204, 220], and 3) percentage volume error rate (percentage of error in volume measurement with respect to the GT's volume) $[187,193,216]$. The mean, standard deviation, and the root-mean-square statistics are often reported for these accuracy/error measures computed for a set of test cases.

Publicly available nodule CT datasets with GT segmentations are an important way to facilitate better validation, especially for cross-algorithm comparisons. In 2003, the Early Lung Cancer Action Program (ELCAP) [177] made a dataset of chest CT scans exhibiting lung nodules available to the public [294]. This dataset has only been used in a few recent studies [201,214]. It was the Lung Image Database Consortium (LIDC) that made the most significant efforts to make such nodule CT datasets with GTs publicly available $[158,295]$. Currently two datasets covering many type of nodules with multiple GT segmentations for each case are available through their website [296], which have already been used by many studies since 2005 [174, 193, 199, 203, 204, 216, 217, 249]. More recently, Italung dataset from a lung cancer screening program in Italy [297, 298] has been used in the studies by Diciotti et al. [193,249]. Despite the increased availability of these public datasets, comprehensive comparison of various methods proposed previously have not yet been thoroughly investigated. Kubota et al. [174] have reported a comparison of five leading methods with the LIDC datasets in their recent report, however more comprehensive comparative studies are necessary for establishing the state-of-the-art in this active research field. 


\section{E. Diagnosis of Lung Nodules}

Once the lung nodules are detected and segmented from the corresponding chest images, the next task is to determine whether the detected nodules are malignant or benign. A number of researchers developed CADx schemes for this task, which distinguish malignant nodules from benign nodules automatically and/or determine the likelihood of malignancy for the detected nodules based on the geometrical size, shape, and appearance of nodules. The performance of schemes were generally evaluated by using the receiveroperating-characteristic (ROC) analysis [299], because this task is two-class classification. Area under the ROC curve (AUC; $A_{z}$ ) [300] was often used as a performance index. Since that the malignancy of lung nodule correlates highly with the geometrical size, shape, and appearance descriptors of the nodules, below, we will review the diagnostic systems that are based on each of these descriptors.

1. Diagnosis of lung nodules based on growth rate

Growth of small pulmonary nodules measured in 2D area [184] and 3D volume [167] has been shown to predict the malignancy in early clinical studies $[167,184]$ and monitoring the tumor response to therapy [169]. More recent clinical lung cancer screening study [301] has demonstrated effectiveness of doubling time measured with commercial nodule segmentation software in diagnosing adenocarcinoma and bronchioloalveolar carcinoma. In oncology, there exists standard clinical protocols for measuring tumor size, such as RECIST and WHO [169]. However, these measures are based on crude linear (sum of $2 \mathrm{D}$ maximal diameters) or bilinear (product of 2D maximal diameter and the largest perpendicular length) approximation of 2D nodule areas, developed prior to the advent of the high-resolution CT technologies. Approximation errors of these 2D measures limits the minimum size of measurable lesions (e.g., $10 \mathrm{~mm}$ in RECIST) because of the wellknown volume averaging effect in that growth of smaller nodules cannot be determined 
with accuracy by them [169]. Addressing this shortcoming, segmentation-based volumetric measures have recently been reported in the clinical literature and actively investigated as alternative to these traditional 2D measures for more accurate size measurement. Accurate volumetric measures as results enable lesion's volumetry: objective quantification of lesion's growth in terms of growth rate (i.e., differentiating nodule's volume measured at different time-points) and/or doubling time (i.e., time that takes to double the volume of a lesion) $[170,248]$.

Aforementioned RECIST and WHO measures involve observer's subjective judgment of 2D lesion boundary and manual measurement of lesion's diameters with a ruler or electronic calipers by oncologists/radiologists [169]. Exercising the same approach to the volumetry would necessitate manual contouring of lesion boundary by the trained observers, which significantly increases their labor burden. Furthermore, the subjective nature of such manual measurements inevitably causes significant intra- and inter-observer variance. In a study comparing various lesion size metrics derived from manually segmented lesion boundaries [289], all 1D, 2D, and 3D/volumetric metrics, included in this study, resulted in high inter-observer variations, mitigating reproducibility of the measures. Reduction of such intra- and inter-observer variance and observer's burden are major motivations for adapting automatic methods for lesion segmentation in order to facilitate objectivity in the volumetry $[222,248]$.

A large volume of recent studies have addressed reproducibility/repeatability of lung nodule volumetry by characterizing its bias and variance with respect to a number of variational factors relevant to current clinical practice and workflows. Factors considered in these studies include nodule characteristics such as size $[188,236,290,292,302$, 303], shape [236, 303], and appearance types of solidness [240]; pulmonary deformation due to cardiovascular motion [304] and inspiration [236]; CT reconstruction parameters such as slice thickness [244, 290, 293, 302,303,305], slice intervals [244, 290, 293, 305], field of views [244, 290, 293, 305] algorithm types [168, 244,305] kernel types [240, 293], 
tube current time [168, 244], and dosage settings [238]; CT scanner vendors [292]; segmentation performance due to choices of threshold parameters in a segmentation algorithm [176,290], segmentation algorithms [168, 176, 291,306], segmentation algorithms in the same segmentation software package [239], segmentation software packages [241], and versions of a segmentation software package [237]; intra- and inter-observer variations by using a commercial software package for semi-automatic nodule segmentation and volumetry [222, 235, 236]; levels of observer's experience and training [291].

Results of these studies have revealed considerable variability of the current volumetric measures when above-listed factors are altered during the volumetric measurement process over time. These variations directly limit the shortest time-interval of CT followup studies to be some large values, reducing its clinical usability [188]. Note that a fixedvalue bias in segmentation error is canceled out when measuring volumetry so that even an inaccurate segmentation algorithm can be a good choice for volumetry as long as it is reproducible and robust [187]. Thus robust segmentation algorithm that produces more consistent results than existing accurate but less robust solutions can be a better choice in this application context [169,207].

a. Technical Approaches Volume measurement precision and accuracy depend on a number of factors, including the inter-scan variability, slice selection artifacts, differences in degree of inspiration and scan angles, and performance of nodule segmentation algorithms, which can make comparing serial scans unreliable. Below, we provide an overview of the existing work on measuring the growth rate of the detected lung nodules.

Generally, the growth rate of pulmonary nodules is determined by a size-based comparison of different temporal CT scans. Earlier 2D techniques exploited changes in the maximal transverse diameter of the nodule to estimate the growth rate between the CT scans $[145,183,184,307,308]$. Unfortunately, these techniques met with problems; for example, possible asymmetric growth results not only in minimal changes in the maximal diameter, but also in an increase of the overall lesion volume [309]. Hence, alternative 3D 
approaches were published for the measurement of the growth rate of small nodules. These volumetric measurements [167, 168, 170, 188, 220, 235, 248, 290, 310-318] have overcome the former 2D limitations.

Yankelevitz et al. [167] used high-resolution CT scans to assess the growth rate of small pulmonary nodules. The region of interest (ROI) containing the nodule was identified in each image manually by a radiologist. Then it was re-sampled to obtain an isotropic space using a 3D linear interpolation, thresholded, and segmented using a 3D technique to reconstruct the 3D image volume. The number of voxels contained in the resulting volume was counted, and the doubling times were estimated using a simple exponential growth model. This $3 \mathrm{D}$ method offered an advantage over the $2 \mathrm{D}$ counterpart that measured the cross-sectional area, but it did not take into account the global motions of the patients due to their movements and the local motions of the whole lung tissues due to breathing and heart beating.

Reeves et al. [248] presented a method for measuring the change in the nodule size from two CT images obtained close in time where the ROI of each CT scan was selected by hand and re-sampled to an isotropic space. To make an accurate assessment and facilitate the comparison of the selected regions, a registration process using the $3 \mathrm{D}$ rigid-body transformation was performed such that both nodules would have the same position and orientation in the image space. Following the registration stage, an adaptive thresholding technique for segmenting the nodule was applied. A rule-based segmentation adjustment was applied to both nodule segmentations. By comparing the nodule segmentations and the thresholded regions, this rule-based system achieved a more consistent measurement of the nodule volumes by discarding missegmented nodule voxels. The main limitation of this work is that only the global motion of the patient, but not the local motion due to breathing and heart beating was taken into account. This strongly affects the estimated growth rate, especially for small detected nodules (less than $5 \mathrm{~mm}$ in diameter).

Taking into account the difference in inspiration levels, Zhao et al. [183] presented 
an adaptive doubling time (ADT) measure of the growth rate of the detected lung nodules. The ADT calculation was obtained through non-rigid lung registration that took into account expanding or shrinking of the nodule. This was accomplished by weighting matching costs of each voxel based on a proposed nodule detection process and a powerful segmentation refinement process. The main limitation of this framework is that the non-rigid registration is applied directly to the segmented nodules. This affects the growth rate estimation because after segmentation of the lung nodules, we can no longer discriminate between the changes due to the true growth rate of the lung nodules and the changes in their shapes that come from breathing and heart beating.

Kawata et al. [311] co-registered the pulmonary nodules by using rigid-body registration and affine registration at two different stages. The nodules were segmented using a 3D deformable surface model, and curvature features were calculated to track the temporal evolution of the nodule. The same research group presented an extension of [311] by adding a 3D non-rigid deformable registration stage, and the analysis was performed using a displacement field to quantify areas of the nodule growth over time [312]. Zheng et al. [220] proposed a simultaneous segmentation and registration of the lung to measure the growth rate from serial CT data. They used a non-rigid transformation for lung deformation and rigid structure for the tumor in order to preserve the volume and the shape of the tumor during the registration. Segmentation of the 3D lung and tumor was based on a 2D graph-cut algorithm, and a B-spline-based non-rigid registration was used. Both these works have the same limitation as the above-mentioned work of Zhao et al. [183].

Jirapatnakul et al. [313] presented a nodule growth measurement method, called growth analysis from density (GAD). They applied a Gaussian weighting function to the region around the nodule to reduce the influence of structures lying far from the nodule center. Also, some researchers used a number of commercial packages that have been released by the CT vendors for measuring the volume of pulmonary nodules, and a number of studies have evaluated the accuracy and limitations of these software packages. Since the 
actual volumes of real pulmonary nodules are unknown, such evaluations usually involve either radiologists/experts as the "gold standard" [235,314-317] or synthetic phantoms for which the volumes of the nodules are known [168,290,318]. A general limitation of the majority of the volumetric measurement algorithms is that they are only capable of segmenting solid nodules. Moreover, the results from these packages show that the volumetric error depends on the performance of the segmentation algorithms, particularly in the presence of the nodules vascular and pleural attachments [170].

Table 7 briefly overviews the different growth rate techniques. In summary, several aspects of growth rate techniques should have further investigations. One of these aspects is to consider the global motion of the patients due to their movements and the local motions of the whole lung tissues due to breathing and heart beating in volumetric measurements of growth rate. Another aspect is that the application of global and local registration directly to the segmented nodule lead to inability to discriminate between the changes due to the true growth of the lung nodules and the changes in the nodule shape which come from breathing and heart beating. Finally, special types of lung nodules such as cavities and ground-glass nodules can not be diagnosed using the current growth-rate techniques, so further methods and nodule descriptors are needed.

TABLE 7: Growth rate based methodologies for following up pulmonary nodules.

\begin{tabular}{|c|c|c|c|c|}
\hline Study & Purpose & Method & Database & Observations \\
\hline $\begin{array}{l}\text { Yankelevitz } \\
\text { et } \\
\text { al. }[184]\end{array}$ & $\begin{array}{l}\text { To assess using } \\
\text { early CT repeat } \\
\text { to determine } \\
\text { nodule growth } \\
\text { rate }\end{array}$ & $\begin{array}{l}\text { 2D growth rate } \\
\text { technique by mea- } \\
\text { suring the maximal } \\
\text { diameter of nodule }\end{array}$ & $\begin{array}{l}\text { Repeat CT for } 15 \\
\text { patients }(15 \text { nod- } \\
\text { ules: } 9 \text { malig- } \\
\text { nant, } 6 \text { benign }(5- \\
20 \mathrm{~mm}))+ \text { spher- } \\
\text { ical phantoms of } \\
\text { known diameters }\end{array}$ & $\begin{array}{l}\text { A single repeat after } 30 \text { days } \\
\text { detect as small as } 5 \mathrm{~mm} \text { ma- } \\
\text { lignant nodule; all the } 15 \\
\text { nodules are correctly classi- } \\
\text { fied }\end{array}$ \\
\hline
\end{tabular}


TABLE 7 - continued from the previous page

\begin{tabular}{|c|c|c|c|c|}
\hline Study & Purpose & Method & Database & Observations \\
\hline $\begin{array}{l}\text { Yankelevitz } \\
\text { et } \\
\text { al. [167] }\end{array}$ & $\begin{array}{l}\text { To determine } \\
\text { the accuracy of } \\
\text { CT volumetric } \\
\text { measurements } \\
\text { of small pul- } \\
\text { monary nodules } \\
\text { to assess growth } \\
\text { and malignancy } \\
\text { status. }\end{array}$ & $\begin{array}{l}\text { Exponential } \\
\text { growth model } \\
\text { to estimate the } \\
\text { doubling time }\end{array}$ & $\begin{array}{l}13 \text { patients } \\
\text { (nodule }<10 \mathrm{~mm}) \\
(5 \text { malignant } \\
\text { and } 8 \text { benign) }+ \\
\text { synthetic phan- } \\
\text { toms of spherical, } \\
\text { defomable, and } \\
\text { different shape } \\
\text { and sizes }\end{array}$ & $\begin{array}{l}\text { (a) The synthetic nodule } \\
\text { studies revealed that the vol- } \\
\text { ume could be measured ac- } \\
\text { curately to within } 3 \% \text {. (b) } \\
\text { All five malignant nodules } \\
\text { grew, and all had doubling } \\
\text { times less than } 177 \text { days. (c) } \\
\text { All eight benign nodules had } \\
\text { doubling times of } 396 \text { days } \\
\text { or greater or showed a de- } \\
\text { crease in volume. }\end{array}$ \\
\hline $\begin{array}{l}\text { Winer- } \\
\text { Muram et } \\
\text { al. [315] }\end{array}$ & $\begin{array}{l}\text { To determine } \\
\text { the range of } \\
\text { growth rates } \\
\text { of stage I lung } \\
\text { cancers prior to } \\
\text { treatment. }\end{array}$ & $\begin{array}{l}\text { Volumetric mea- } \\
\text { surement }\end{array}$ & $\begin{array}{l}50 \text { patients, } 50 \text { tu- } \\
\text { mor }\end{array}$ & $\begin{array}{l}\text { Comparison of tumor vol- } \\
\text { umes at serial CT exami- } \\
\text { nations reveals a very wide } \\
\text { range of growth rates. Some } \\
\text { tumors grow so slowly that } \\
\text { biopsy is required to prove } \\
\text { they are malignant. }\end{array}$ \\
\hline $\begin{array}{l}\text { Borghesi } \\
\text { et } \\
\text { al. [309] }\end{array}$ & $\begin{array}{l}\text { To evaluate } \\
\text { the accuracy } \\
\text { of software- } \\
\text { calculated } \\
\text { growth rate of } \\
\text { small nodules } \\
\text { in detecting } \\
\text { malignancy }\end{array}$ & $\begin{array}{l}\text { Volume doubling } \\
\text { time was calcu- } \\
\text { lated on Aquarius } \\
\text { Workstation (Ter- } \\
\text { aRecon, Inc.) with } \\
\text { the segmenta- } \\
\text { tion analysis and } \\
\text { tracking (SAT) } \\
\text { module. }\end{array}$ & $\begin{array}{l}29 \text { patients (40 } \\
\text { nodules (solid } \\
\text { or noncalcified) } \\
4-15 \text { mm, glass } \\
\text { opacities nodules } \\
\text { were discarded); } \\
4 \text { of the nodules } \\
\text { are given their di- } \\
\text { agnosis (3 benign } \\
\text { and } 1 \text { malignant). }\end{array}$ & $\begin{array}{l}4 \text { nodules are correctly clas- } \\
\text { sified }\end{array}$ \\
\hline $\begin{array}{l}\text { Wormanns } \\
\text { et } \\
\text { al. }[235]\end{array}$ & $\begin{array}{l}\text { To assess the } \\
\text { measurement } \\
\text { precision of a } \\
\text { software tool } \\
\text { for volumetric } \\
\text { analysis of } \\
\text { nodules from } \\
\text { two consecutive } \\
\text { low-dose CT } \\
\text { scans. }\end{array}$ & $\begin{array}{l}\text { Volumetric mea- } \\
\text { surement }\end{array}$ & $\begin{array}{l}10 \text { subject, } 151 \\
\text { nodules }\end{array}$ & $\begin{array}{l}\text { Taking into account all } \\
151 \text { nodules, } 95 \% \text { limits } \\
\text { of agreement were }-20.4 \\
\text { to } 21.9 \% \text { (standard error } \\
1.5 \% \text { ). }\end{array}$ \\
\hline $\begin{array}{l}\text { Revel et } \\
\text { al. [314] }\end{array}$ & $\begin{array}{l}\text { To evaluate soft- } \\
\text { ware designed } \\
\text { to calculate pul- } \\
\text { monary nodule } \\
\text { volume in 3D. }\end{array}$ & $\begin{array}{l}\text { Volumetric mea- } \\
\text { surement }\end{array}$ & $\begin{array}{l}54 \text { nodules, } 22 \text { di- } \\
\text { agnosed: } 13 \text { be- } \\
\text { nign, } 9 \text { malignant }\end{array}$ & $\begin{array}{l}\text { Software measurement error } \\
\text { of } 6.38 \% \text { of the previous } \\
\text { volume measurement. }\end{array}$ \\
\hline \multicolumn{5}{|r|}{ continued on the next page ... } \\
\hline
\end{tabular}


TABLE 7 - continued from the previous page

\begin{tabular}{|c|c|c|c|c|}
\hline Study & Purpose & Method & Database & Observations \\
\hline $\begin{array}{l}\text { Kostis et } \\
\text { al. }[188]\end{array}$ & $\begin{array}{l}\text { To determine } \\
\text { the repro- } \\
\text { ducibility of } \\
\text { volume mea- } \\
\text { surements of } \\
\text { small pul- } \\
\text { monary nodules } \\
\text { on CT scans } \\
\text { and to estimate } \\
\text { critical time to } \\
\text { follow-up CT. }\end{array}$ & $\begin{array}{l}\text { Percentage volume } \\
\text { change (PVC) and } \\
\text { monthly volumet- } \\
\text { ric growth index } \\
\text { (MVGI) were } \\
\text { computed for each } \\
\text { nodule pair. }\end{array}$ & 115 nodule & $\begin{array}{l}\text { Factors that affect repro- } \\
\text { ducibility of nodule volume } \\
\text { measurements and critical } \\
\text { time to follow-up CT in- } \\
\text { clude nodule size at detec- } \\
\text { tion, type of scan (baseline } \\
\text { or repeat) on which the nod- } \\
\text { ule is detected, and presence } \\
\text { of patientinduced artifacts. }\end{array}$ \\
\hline $\begin{array}{l}\text { Goo et } \\
\text { al. [290] }\end{array}$ & $\begin{array}{l}\text { To evaluate } \\
\text { the effect of } \\
\text { CT parameters } \\
\text { and nodule } \\
\text { segmentation } \\
\text { thresholds on } \\
\text { accuracy of } \\
\text { volumetric } \\
\text { measurement of } \\
\text { synthetic lung } \\
\text { nodules. }\end{array}$ & $\begin{array}{l}\text { Volumetric mea- } \\
\text { surement }\end{array}$ & $\begin{array}{l}4 \text { types of Lung } \\
\text { phantoms }\end{array}$ & $\begin{array}{l}\text { For accurate measurement } \\
\text { of lung nodule volume, it } \\
\text { is critical to select a sec- } \\
\text { tion thickness and/or seg- } \\
\text { mentation threshold appro- } \\
\text { priate for the size of a nod- } \\
\text { ule. }\end{array}$ \\
\hline $\begin{array}{l}\text { Reeves } \\
\text { al. }[248]\end{array}$ & $\begin{array}{l}\text { To develop } \\
\text { a method for } \\
\text { measuring the } \\
\text { change in nod- } \\
\text { ule size from } 2 \\
\text { CT image scans } \\
\text { recorded at } \\
\text { different times } \\
\text { to establish the } \\
\text { growth rate. }\end{array}$ & $\begin{array}{l}\text { Registration, adap- } \\
\text { tive thresholding, } \\
\text { and knowledge- } \\
\text { based shape } \\
\text { matching }\end{array}$ & $\begin{array}{l}50 \text { benign or } \\
\text { 2YNC nodule }\end{array}$ & $\begin{array}{l}\text { The variance in percent } \\
\text { volume change was reduced } \\
\text { from } 11.54 \% \text { to } 9.35 \% \\
\text { through the use of registra- } \\
\text { tion, adaptive thresholding, } \\
\text { and knowledge-based shape } \\
\text { matching. }\end{array}$ \\
\hline $\begin{array}{l}\text { Jennings } \\
\text { et } \\
\text { al. [316] }\end{array}$ & $\begin{array}{l}\text { To retrospec- } \\
\text { tively determine } \\
\text { the distribution } \\
\text { of stage I lung } \\
\text { cancer growth } \\
\text { rates with serial } \\
\text { volumetric CT }\end{array}$ & $\begin{array}{l}\text { Volumetric mea- } \\
\text { surement }\end{array}$ & 149 patients & $\begin{array}{l}\text { At serial volumetric CT } \\
\text { measurements, there was } \\
\text { wide variability in growth } \\
\text { rates. Some biopsy-proved } \\
\text { cancers decreased in volume } \\
\text { between examinations }\end{array}$ \\
\hline $\begin{array}{l}\text { Zheng et } \\
\text { al. [220] }\end{array}$ & $\begin{array}{l}\text { To simultane- } \\
\text { ously segment } \\
\text { and register } \\
\text { lung and tumor } \\
\text { in serial CT } \\
\text { data. }\end{array}$ & $\begin{array}{l}\text { Nonrigid transfor- } \\
\text { mation on lung de- } \\
\text { formation and rigid } \\
\text { structure on the tu- } \\
\text { mor. }\end{array}$ & $\begin{array}{l}6 \text { volume of } 3 \text { pa- } \\
\text { tients, } 83 \text { nodule }\end{array}$ & $\begin{array}{l}\text { The mean error of bound- } \\
\text { ary distances between auto- } \\
\text { matic segmented boundaries } \\
\text { of lung tumors and man- } \\
\text { ual segmentation is } 3.50 \text { pix- } \\
\text { els. The mean and variance } \\
\text { of percentages of the nodule } \\
\text { volume variations caused by } \\
\text { errors in segmentation, are } \\
0.8 \text { and } 0.6 \text {. }\end{array}$ \\
\hline
\end{tabular}


TABLE 7 - continued from the previous page

\begin{tabular}{|c|c|c|c|c|}
\hline Study & Purpose & Method & Database & Observations \\
\hline $\begin{array}{l}\text { Jirapatnak } \\
\text { et } \\
\text { al. }[313]\end{array}$ & $\begin{array}{l}\text { ITo measures the } \\
\text { nodule growth } \\
\text { without explicit } \\
\text { segmentation }\end{array}$ & $\begin{array}{l}\text { Growth analy- } \\
\text { sis from density } \\
\text { (GAD) method } \\
\text { to measure the } \\
\text { growth rate }\end{array}$ & $\begin{array}{l}20 \text { cases each } \\
\text { with single nod- } \\
\text { ule with scans } \\
\text { several minutes } \\
\text { apart (expected } \\
\text { zero volume } \\
\text { change), } 38 \text { cases } \\
\text { with a stable } \\
\text { nodule, } 19 \text { cases } \\
\text { with a malignant } \\
\text { nodule, and } 4 \text { ma- } \\
\text { lignant nodules } \\
\text { with a complex } \\
\text { appearance }\end{array}$ & $\begin{array}{l}\text { Accuracy achieved was } \\
\text { ( } 37 / 38 \text { for the stable benign } \\
\text { nodules), ( } 18 / 19 \text { for the ma- } \\
\text { lignant nodules), and ( } 4 / 4 \\
\text { for the complex malignant } \\
\text { nodules) }\end{array}$ \\
\hline $\begin{array}{l}\text { Marchianò } \\
\text { et } \\
\text { al. [317] }\end{array}$ & $\begin{array}{l}\text { To assess in } \\
\text { vivo volumetric } \\
\text { repeatability of } \\
\text { an automated } \\
\text { algorithm for } \\
\text { volume estima- } \\
\text { tion. }\end{array}$ & $\begin{array}{l}\text { Semiautomatic } \\
\text { volumetric mea- } \\
\text { surement }\end{array}$ & $\begin{array}{l}101 \text { subject, } 233 \\
\text { nodules }\end{array}$ & $\begin{array}{l}\text { The } 95 \% \text { confidence interval } \\
\text { for difference in measured } \\
\text { volumes was in the range of } \\
+-27 \% \text {. About } 70 \% \text { of mea- } \\
\text { surements had a relative dif- } \\
\text { ference in nodule volume of } \\
\text { less than } 10 \% \text {. }\end{array}$ \\
\hline
\end{tabular}

2. Diagnosis of lung nodules based on shape and appearance

A great deal of work has been published regarding the usefulness of morphologic features to distinguish between malignant and benign pulmonary nodules on computed tomography (CT) and, to a lesser extent, chest radiographs. Several studies have shown a correlation between different nodule shape characteristics and their underlying pathology. For example, Furuya et al. [319] analyzed the margin characteristics of 193 pulmonary nodules on high-resolution CT scans and subjectively classified them as one of several types, including round, lobulated, densely spiculated, ragged, and halo. They found a high level of malignancy among the lobulated (82\%), spiculated (97\%), ragged (93\%), and halo nodules (100\%), while $66 \%$ of the round nodules proved to be benign.

Automatically extracted features have also been shown to correlate with underlying malignancy. Kawata et al. $[172,195]$ quantified the surface curvature and degree of surrounding radiating patterns in biopsy-proven benign and malignant nodules when com- 
pared with the resulting feature maps. Their results showed good separation of the feature maps between the two categories. Their further work [320] extended the curvature analysis method to include internal nodule features, and using this method, which is described in more detail below, they attained similar results. Similarly, fractal analysis has been used to quantify the nodule margin characteristics of benign and malignant nodules. The same research group [321] designed an automated retrieval system to obtain diagnosis and prognosis information by searching similar images in a 3D CT image database of pulmonary nodules (248 nodule, 179 malignant and 69 benign) for which diagnosis is known. A region of interest (ROI) is selected to include the nodule region and its surrounding. Each voxel in the ROI is represented using its CT density and a curvature shape index. The CT density and shape index are characterized using joint histograms for analysis. For each input nodule, a similarity measure between the input nodule and the database is estimated using the correlation coefficient of the joint histograms of the nodules. The results for queering the $3 \mathrm{D}$ database for similar nodules show a reasonably set of similar nodules sorted from highest to lowest similarity with the queried nodule. Kido et al. [322] used 2D and 3D fractal dimensions to analyze the lung-nodule interface in a series of 117 peripheral pulmonary nodules with various underlying pathology, including benign hamartomas, tuberculomas, and pneumonias, as well as malignant diagnoses including brochogenic carcinomas. They noted statistically significant differences between the 2D fractal dimensions of hamartomas and all other nodules, as well as differences between the 3D fractal dimensions of pneumonias and tuberculomas and bronchogenic carcinomas. Although none of these studies directly assessed the accuracy of their methods for diagnosis prediction, they supported the notion that the nodule shape can potentially be used by automated systems to distinguish between benign and malignant nodules.

Severalgroups have designed Computer Aided Diagnostic (CAD) systems with the goal of predicting a diagnosis based on features extracted from CT scans or chest radiographs. In general, these systems share the following common schema: first extracting 
features from the images, then designing and using an automatic classifier to categorize nodules based on these features, and lastly, evaluating the performance of the system with Receiver Operating Characteristics (ROC) analysis. The CAD systems differ in the specific extracted features and the type of classifier used, with Linear Discriminant Classifiers (LDC) and neural networks (NN) being the most common. Below, systems based on LDC classifiers will be discussed followed by systems based on NN and other types of classifiers.

Kawata and colleagues [323]designed a CT-based CAD system that classified pulmonary nodules based on a combination of curvature index and the relationship of the nodules to their surrounding features. The curvature index of a nodule is calculated from a combination of shape indices, which describe the surface type (i.e. ridge, saddle, pit, etc), and curvedness, which describes the degree of curvature. The area surrounding the nodules was assessed for degree of vascular convergence and pleural retraction using vector field analysis. Using an LDC classifier based on these features to evaluate a series of 248 nodules (179 malignant and 69 benign), they found the combination of curvature-based and surrounding features to be most accurate (area under ROC curve $\left(A_{z}=0.94\right)$ ), followed by curvature-based alone $\left(A_{z}=0.88\right)$, and surrounding characteristics alone $\left(A_{z}=0.69\right)$. Mori et al. [324] also designed a CAD system using curvedness index in combination with dynamic contrast-enhanced CT in order to evaluate temporal change as a possible discriminating feature of benign and malignant nodules. Shape index, curvedness values, and attenuation were calculated at 0,2 , and 4 minutes after contrast administration, and using these values, a score was generated by an LDC. Attenuation had an $A_{z}$ value of 0.69 at 2 minutes post-contrast, the highest of the three time points. Curvedness yielded a maximum $A_{z}$ of 0.83 at 2 minutes, and shape index had an $A_{z}$ value of 0.90 at 0 and 2 minutes. The combination of all three features had an $A_{z}$ value of 1.00 at 4 minutes.

The CAD system developed by McNitt-Gray et al. [308] used a pattern classification approach to determine the malignancy of pulmonary nodules on HRCT in a series of 31 cases (17 malignant, 14 benign). They identified solitary nodules using a semi-automated 
contouring technique, and extracted quantitative measures of the resulting contour related to shape, size, attenuation, distribution of attenuation and texture. Using a stepwise discriminant analysis, they selected features that were best able to predict malignancy and used these to design a LDC to characterize the nodules. The selected features predicted malignancy with an accuracy of $90.3 \%$ (28/31), however no $A_{z}$ value was reported.

Shahet al. [325] designed a CAD system that extracted features from two separate contours, one including only the solid portion of the nodule and one including any groundglass components. For each contour, 75 features were calculated to measured nodule attenuation, shape, and texture. These features were then input into a feature selection step, and four different classifiers were used to determine if the diagnosis could be predicted from the feature vector. Training and testing was conducted using both re-substitution and leaveone-out methods. With leave-one-out testing methodology with a database composed of 19 malignant and 16 benign nodules, the classifiers resulted with an $A_{z}$ ranging from 0.68 to 0.92. When evaluating with re-substitution, the $A_{z}$ ranged from 0.93 to 1.00 . The same research group [326] employed different classifiers such as logistic regression and quadratic discriminant analysis (QDA) with features selected from 31 features by using stepwise feature selection based on Akaike information criterion. Their scheme with logistic regression achieved an AUC value of 0.92 in distinction between 19 malignant and 16 benign nodules in thin-slice CE-CT.

Other LDC based CAD systems include those developed by Way and colleagues [199]. They designed a system based on morphologic and texture features of pulmonary nodules on CT images, using a series of 96 lung nodules, with 44 biopsy or PET scan proven malignant nodules and 52 nodules that proved to be benign on biopsy or follow-up CT. The nodules were segmented using 3D active contours that were guided by a combination of 2D and 3D energies. Next, they extracted several morphologic and texture based features from the segmented nodules. Morphologic features include volume, surface area, perimeter, maximum diameter, and maximum and minimum $\mathrm{CT}$ value inside the nodule. Using a 
stepwise method, they selected the most predictive features for use in the LDC. The classifier was trained and tested using a leave-one-out method, and the system achieved an $A_{z}$ of 0.83. More recently, the same group [327] designed a system using the morphologic features described above in combination with new measurements of the surface characteristics that quantified the smoothness and shape irregularity of the nodules. They calculated ROC statistics for LDCs designed with and without the new surface features, and found a significant $(p<0.05)$ improvement in performance with the $A_{z}$ increasing from 0.821 to 0.857 in classification of 124 malignant and 132 benign nodules in 152 patients. Aoyama et al. [328] used LDC for distinction between malignant and benign nodules in thick-slice screening LDCT. They achieved an AUC value of 0.846 for a database of 73 patients with 76 primary cancers and 342 patients with 413 benign nodules.

One of the early neural network based CAD systems was developed by Gurney and Swensen [329]. They compared two systems, one using a neural network based classifier and one using a Bayesian classifier. Both systems used a combination of subjectively evaluated clinical and radiologic characteristics including border smoothness, speculation, and lobulation. The Bayesian system showed a significantly $(p<0.05)$ higher level of performance $\left(A_{z}=0.894\right)$ than the neural network based system $\left(A_{z}=0.871\right)$. Another neural network based system using subjectively extracted features was developed by Matsuki et al. [330]. Radiologic features included shape-based parameters such as border definition, spiculation, and concavity as well as other associated features such as blood vessel involvement, lymphadenopathy, and emphysematous changes. From a series of 155 nodules found on HRCT (99 malignant, 56 benign), features were extracted by attending radiologists using subjective rating scales and used to train the neural network. The neural network alone showed a high level of performance $\left(A_{z}=0.951\right)$, and significantly increased the radiologists' performance, increasing the $A_{z}$ value from 0.831 to 0.959 .

Other CAD systems have been designed to automatically define and extract features as well as classify nodules. For example, Henschke et al. [331] adapted the S-MODALS 
neural network, originally designed for tactical and strategic reconnaissance, to the task of nodule classification. Features were automatically selected from the example image using a NN clustering technique with operator-defined selection parameters including spatial separation of features and the degrees of similarity and dissimilarity that grouped features in to clusters. The system was tested on a series of 28 biopsy-proven nodules (14 malignant, 14 benign), and all but 3 benign nodules were correctly classified. Another neural network using automatically extracted features was designed by Lo et al. [332], and used a combination of radiographic parameters including vascularity, CT density distribution, and shape indices including aspect ratio, circularity, irregularity, extent, compactness, and convexity. Nodules were segmented using an automatic thresholding method, and the resulting $3 \mathrm{D}$ volumes were automatically smoothed and pruned of vasculature. The vascular index was calculated during this smoothing process, and shape indices were calculated from the resulting volume. Using a leave-one-out method, they trained the neural network on a series of 48 nodules ( 24 malignant, 24 benign). The results yielded an $A_{z}$ value of 0.89 , and they found that the most predictive features were the vascular index, size, compactness, and difference entropy of the CT density.

Suzuki et al. [139] developed a multiple MTANN scheme for the classification task based on training the MTANN classifier with a set of benign and malignant nodules. They achieved an AUC value of 0.88 for thick-slice screening low-dose (LD) CT scans of 73 patients with 76 primary cancers and 342 patients with 413 benign nodules. Chen et al. [333] employed ANN ensemble to classify 19 malignant and 13 benign nodules, and they achieved an AUC value of 0.915 .

Nakamura et al. [334] compared the performance of two separate networks, one trained on 8 subjective features rated by radiologists (i.e., nodule size, shape (round -toelongated), marginal irregularity, spiculation, border definition, lobulation, nodule density (contrast)) and the other trained on 12 matched features automatically extracted from chest radiographs (i.e., effective diameter, degree of circularity, degree of ellipticity, magnitude 
and coarseness of irregular edge patterns, mean gradient, radial gradient index, tangential gradient index, mean pixel, line enhancement index). Both sets included shape-based features including margin irregularity, spiculation, lobulation, and nodule shape as well measures of homogeneity and CT density. The network based on objective features demonstrated the highest level of performance $\left(A_{z}=0.854\right)$, and was followed by the subjective feature network $\left(A_{z}=0.761\right)$, and then the radiologists $\left(A_{z}=0.752\right)$.

Iwano et al. [335] developed a system to automatically classify pulmonary nodules detected on HRCT into different shape categories, and compared the performance to radiologists. The nodules were extracted from a series of 102 CT images without a prior diagnosis of malignancy, and were classified into different shape categories based on quantitative measures of aspect ratio, circularity, and their second central moment. The results were compared to a subjective classification by radiologists, and they found that the automated system classified the nodules as accurately as the radiologists. Although no direct attempt at automatic diagnosis was carried out, they concluded that system had the potential to aid radiologists in classifying nodules as malignant or benign based on the correlation between certain shape categories and underlying pathology. The same search group [336] extended their work on 107 HRCT images and achieved a sensitivity of $76.9 \%$ and a specificity of $80 \%$ with their scheme based on LDA with 2 features (circularity and second moment) in classification of total of 52 malignant and 55 benign nodules.

TABLE 8: Classification between malignant and benign nodules based on shape and appearance features.

\begin{tabular}{|l|l|l|l|l|}
\hline \multicolumn{1}{|c|}{ Study } & \multicolumn{1}{|c|}{ Purpose } & \multicolumn{1}{c|}{ Method } & \multicolumn{1}{c|}{ Database } & \multicolumn{1}{c|}{ Observations } \\
\hline $\begin{array}{l}\text { Kawata } \\
\text { et } \\
\text { al. [172] }\end{array}$ & $\begin{array}{l}\text { To surface } \\
\text { acterize } \\
\text { morphology } \\
\text { of small } \\
\text { pulmonary } \\
\text { nodules }\end{array}$ & $\begin{array}{l}\text { Using } \\
\text { curvatures and } \\
\text { ridge line. }\end{array}$ & $\begin{array}{l}\text { Thin-section CT im- } \\
\text { ages for 56 cases in- } \\
\text { cluding 42 malignant } \\
\text { and 14 benign nod- } \\
\text { ules }\end{array}$ & $\begin{array}{l}\text { The distribution of the nod- } \\
\text { characteristics in the } \\
\text { feature space shows good } \\
\text { evidence of separation be- } \\
\text { tween the two classes. }\end{array}$ \\
\hline
\end{tabular}


TABLE 7 - continued from the previous page

\begin{tabular}{|c|c|c|c|c|}
\hline Study & Purpose & Method & Database & Observations \\
\hline $\begin{array}{l}\text { Henschke } \\
\text { et } \\
\text { al. }[331]\end{array}$ & $\begin{array}{l}\text { To explore } \\
\text { the usefulness } \\
\text { of neural net- } \\
\text { works (NNs) } \\
\text { to help in this } \\
\text { differentia- } \\
\text { tion. }\end{array}$ & $\begin{array}{l}\text { Statistical- } \\
\text { multiple object } \\
\text { detection and } \\
\text { location system } \\
\text { (S-MODALS) } \\
\text { NN technique } \\
\text { developed for } \\
\text { automatic tar- } \\
\text { get recognition } \\
\text { (ATR) }\end{array}$ & $\begin{array}{l}\text { CT images of } 28 \text { pul- } \\
\text { monary nodules, } 14 \\
\text { benign and } 14 \text { malig- } \\
\text { nant, each having a } \\
\text { diameter less than } 3 \\
\text { cm were selected. }\end{array}$ & $\begin{array}{l}\text { Correctly identify all but } \\
\text { three benign nodules, but } \\
\text { did not misclassify any ma- } \\
\text { lignant nodules. }\end{array}$ \\
\hline $\begin{array}{l}\text { Kawata } \\
\text { et } \\
\text { al. [195] }\end{array}$ & $\begin{array}{l}\text { To charac- } \\
\text { terize the } \\
\text { internal struc- } \\
\text { ture of small } \\
\text { pulmonary } \\
\text { nodules }\end{array}$ & $\begin{array}{l}\text { Using multi-scale } \\
\text { curvature-based } \\
\text { shape spectrum }\end{array}$ & $\begin{array}{l}\text { Thin-section CT im- } \\
\text { ages of } 27 \text { pulmonary } \\
\text { nodules ( } 9 \text { solid be- } \\
\text { nign and } 18 \text { solid and } \\
\text { infiltrative malignant } \\
\text { cases). }\end{array}$ & $\begin{array}{l}\text { The distribution of the nod- } \\
\text { ules characteristics in the } \\
\text { feature space shows good } \\
\text { evidence of separation be- } \\
\text { tween the two classes. }\end{array}$ \\
\hline $\begin{array}{l}\text { McNitt- } \\
\text { Gray et } \\
\text { al. }[308]\end{array}$ & $\begin{array}{l}\text { To classify } \\
\text { nodules into } \\
\text { benign or } \\
\text { malignat }\end{array}$ & $\begin{array}{l}\text { LDA with step- } \\
\text { wise feature se- } \\
\text { lection based on } \\
\text { nodule's shape, } \\
\text { size, attenuation, } \\
\text { distribution of } \\
\text { attenuation, and } \\
\text { texture }\end{array}$ & $\begin{array}{l}\text { HRCT scans of } 17 \\
\text { malignant and } 14 \text { be- } \\
\text { nign nodules }\end{array}$ & $\begin{array}{l}\text { Correct classification rate of } \\
90.3 \%\end{array}$ \\
\hline $\begin{array}{l}\text { Kawata } \\
\text { et } \\
\text { al. [323] }\end{array}$ & $\begin{array}{l}\text { To discrimi- } \\
\text { nate between } \\
\text { benign and } \\
\text { malignant } \\
\text { nodules }\end{array}$ & $\begin{array}{l}\text { LDA with step- } \\
\text { wise feature } \\
\text { selection based } \\
\text { on nodule's fea- } \\
\text { tures (density } \\
\text { and curvatures) } \\
\text { and surrounding } \\
\text { structure features. }\end{array}$ & $\begin{array}{l}\text { CT images of } 248 \\
\text { pulmonary nodules } \\
\text { including } 179 \text { malig- } \\
\text { nant and } 69 \text { benign } \\
\text { nodules. }\end{array}$ & $\begin{array}{l}\text { Nodule's } \\
(\mathrm{Az}=\mathrm{O} .88) \text { were more } \\
\text { effective than the sur- } \\
\text { rounding structure features } \\
\text { (Az=O. 69) in classification. } \\
\text { Combing both features } \\
\text { achieves } A z=0.94 \text {. }\end{array}$ \\
\hline $\begin{array}{l}\text { Kawata } \\
\text { et } \\
\text { al. }[321]\end{array}$ & $\begin{array}{l}\text { To obtain } \\
\text { nodule di- } \\
\text { agnosis } \\
\text { information } \\
\text { by image } \\
\text { retrieval from } \\
\text { a database } \\
\text { of known } \\
\text { diagnosis. } \\
\end{array}$ & $\begin{array}{l}\text { Retrieving the } \\
\text { nodules with } \\
\text { similar character- } \\
\text { istics from a 3D } \\
\text { Image database } \\
\text { based on its } \\
\text { CT density and } \\
\text { curvature index. }\end{array}$ & $\begin{array}{l}\text { CT images of } 248 \\
\text { pulmonary nodules } \\
\text { including } 179 \text { malig- } \\
\text { nant and } 69 \text { benign } \\
\text { nodules. }\end{array}$ & $\begin{array}{l}\text { The resulted visual figures } \\
\text { are sorted from more simi- } \\
\text { lar to less similar with ma- } \\
\text { lignant case and show a high } \\
\text { similarity with the test nod- } \\
\text { ule. }\end{array}$ \\
\hline $\begin{array}{l}\text { Matsuki } \\
\text { et } \\
\text { al. [330] }\end{array}$ & $\begin{array}{l}\text { To classify } \\
\text { nodules into } \\
\text { benign or } \\
\text { malignat }\end{array}$ & $\begin{array}{l}\text { ANN with } 16 \\
\text { subjective fea- } \\
\text { tures determined } \\
\text { by radiologists } \\
\text { and } 7 \text { clinical } \\
\text { data }\end{array}$ & $\begin{array}{l}155 \text { HRCT scans of } \\
99 \text { malignant and } 56 \\
\text { benign nodules }\end{array}$ & $\begin{array}{l}\text { AUC value of } 0.951 \text { in a } \\
\text { leave-one-out test }\end{array}$ \\
\hline \multicolumn{5}{|r|}{ continued on the next page ... } \\
\hline
\end{tabular}


TABLE 7 - continued from the previous page

\begin{tabular}{|c|c|c|c|c|}
\hline Study & Purpose & Method & Database & Observations \\
\hline $\begin{array}{l}\text { Lo et } \\
\text { al. [332] }\end{array}$ & $\begin{array}{l}\text { To quantify } \\
\text { lung nodules } \\
\text { in thoracic CT }\end{array}$ & $\begin{array}{l}\text { A NN based } \\
\text { on geometrical } \\
\text { features, inten- } \\
\text { sity and texture } \\
\text { features. }\end{array}$ & $\begin{array}{l}\text { CT images of } 48 \\
\text { cases of lung nodules } \\
\text { ( } 24 \text { benign, } 24 \text { malig- } \\
\text { nant) }\end{array}$ & $\mathrm{Az}$ is 0.89 \\
\hline $\begin{array}{l}\text { Aoyama } \\
\text { et } \\
\text { al. [328] }\end{array}$ & $\begin{array}{l}\text { To classify } \\
\text { nodules into } \\
\text { benign or } \\
\text { malignat }\end{array}$ & $\begin{array}{l}\text { LDA with Wilks' } \\
\text { lambda stepwise } \\
\text { feature selection }\end{array}$ & $\begin{array}{l}\text { Thick-slice }(10 \mathrm{~mm}) \\
\text { screening } \text { LDCT } \\
\text { scans of } 76 \text { malig- } \\
\text { nant and } 413 \text { benign } \\
\text { nodules }\end{array}$ & $\begin{array}{l}\text { AUC of } 0.846 \text { in a leave- } \\
\text { one-out test }\end{array}$ \\
\hline $\begin{array}{l}\text { Nakamura } \\
\text { al. }[334]\end{array}$ & $\begin{array}{l}\text { To classify } \\
\text { nodules into } \\
\text { benign or } \\
\text { malignat }\end{array}$ & $\begin{array}{l}\text { Two NNs: one } \\
\text { trained with } 8 \\
\text { subjective feature } \\
\text { recorded by } \\
\text { radiologist rating } \\
\text { and the other } \\
\text { with } 12 \text { matched } \\
\text { computerized } \\
\text { objective features }\end{array}$ & $\begin{array}{l}56 \text { radiographs of } 34 \\
\text { malignant and } 22 \text { be- } \\
\text { nign nodules }\end{array}$ & $\begin{array}{l}\mathrm{Az}=0.854 \text { using subjective } \\
\text { features and } \mathrm{Az}=0.761 \text { using } \\
\text { objective features. The re- } \\
\text { ported radiologist accuracy } \\
\text { was } \mathrm{Az}=0.752 \text {. }\end{array}$ \\
\hline $\begin{array}{l}\text { Iwano et } \\
\text { al. [335] }\end{array}$ & $\begin{array}{l}\text { To classify } \\
\text { the shape of } \\
\text { pulmonary } \\
\text { nodules using } \\
\text { computer } \\
\text { analysis of } \\
\text { HRCT }\end{array}$ & $\begin{array}{l}\text { LDA with } 2 \text { fea- } \\
\text { tures (circularity } \\
\text { and second mo- } \\
\text { ment) }\end{array}$ & $\begin{array}{l}\text { HRCT images from } \\
102 \text { patients with } 102 \\
\text { nodules classified as: } \\
\text { round or oval; lobu- } \\
\text { lated; polygonal; ten- } \\
\text { tacluar; spiculated; } \\
\text { ragged; and irregular. }\end{array}$ & $\begin{array}{l}\text { For } 95 \text { of } 102 \text { cases, the } \\
\text { shape classification by the } \\
\text { two radiologists was the } \\
\text { same. For the seven mis- } \\
\text { matched cases, pulmonary } \\
\text { nodules with circularity } \\
\leq 0.75 \text { and second moment } \\
\leq 0.18 \text { were very likely to } \\
\text { reveal lung cancer. }\end{array}$ \\
\hline $\begin{array}{l}\text { Shah et } \\
\text { al. [326] }\end{array}$ & $\begin{array}{l}\text { To classify } \\
\text { nodules into } \\
\text { benign or } \\
\text { malignat }\end{array}$ & $\begin{array}{l}\text { Logistic regres- } \\
\text { sion or QDA with } \\
\text { stepwise feature } \\
\text { selection from } 31 \\
\text { features }\end{array}$ & $\begin{array}{l}\text { Thin-slice }(\leq 3 \mathrm{~mm}) \\
\text { CE-CT scans of } 19 \\
\text { malignant and } 16 \text { be- } \\
\text { nign nodules }\end{array}$ & $\begin{array}{l}\text { AUC of } 0.69 \text { and } 0.92 \\
\text { with logistic regression } \\
\text { and QDA, respectively in a } \\
\text { leave-one-out test }\end{array}$ \\
\hline $\begin{array}{l}\text { Matsuoka } \\
\text { et } \\
\text { al. [337] }\end{array}$ & $\begin{array}{l}\text { To analyze } \\
\text { features of } \\
\text { peripheral } \\
\text { noncalci- } \\
\text { fied solitary } \\
\text { pulmonary } \\
\text { nodules in } \\
\text { patients with } \\
\text { emphysema. }\end{array}$ & $\begin{array}{l}\text { Analyze the frac- } \\
\text { tal dimensions } \\
\text { of the nodule } \\
\text { interfaces, nodule } \\
\text { circularity, and } \\
\text { the percentage } \\
\text { of the nodule } \\
\text { surrounded by } \\
\text { emphysema. }\end{array}$ & $\begin{array}{l}\text { CT images of } 41 \\
\text { nodules ( } 21 \text { malig- } \\
\text { nant, } 20 \text { benign }) \text { in } \\
41 \text { patients with em- } \\
\text { physema }\end{array}$ & $\begin{array}{l}\text { In patients with emphysema, } \\
\text { there were no significant dif- } \\
\text { ferences in fractal dimen- } \\
\text { sion, circularity, or fre- } \\
\text { quency of lobulation or spic- } \\
\text { ulation between malignant } \\
\text { and benign nodules. }\end{array}$ \\
\hline $\begin{array}{l}\text { Mori et } \\
\text { al. [324] }\end{array}$ & $\begin{array}{l}\text { To classify } \\
\text { nodules into } \\
\text { benign or } \\
\text { malignat }\end{array}$ & $\begin{array}{l}\text { LDA using } 3 \text { fea- } \\
\text { tures: Shape in- } \\
\text { dex, curvedness } \\
\text { values, and atten- } \\
\text { uation. }\end{array}$ & $\begin{array}{l}\text { Thin-slice }(2 \mathrm{~mm}) \\
\text { CE-CT scans of } 35 \\
\text { malignant and } 27 \text { be- } \\
\text { nign nodules }\end{array}$ & $\begin{array}{l}\text { AUC of } 0.91 \text { and } 1.0 \text { with } \\
\text { non-CE CT and CE-CT, re- } \\
\text { spectively in a leave-one-out } \\
\text { test }\end{array}$ \\
\hline \multicolumn{5}{|r|}{ continued on the next page ... } \\
\hline
\end{tabular}


TABLE 7 - continued from the previous page

\begin{tabular}{|c|c|c|c|c|}
\hline Study & Purpose & Method & Database & Observations \\
\hline $\begin{array}{l}\text { Suzuki et } \\
\text { al. [139] }\end{array}$ & $\begin{array}{l}\text { To classify } \\
\text { nodules into } \\
\text { benign or } \\
\text { malignat }\end{array}$ & $\begin{array}{l}\text { Multiple } \\
\text { MTANNs using } \\
\text { pixel values in a } \\
9 \times 9 \text { subregion }\end{array}$ & $\begin{array}{l}\text { Thick-slice }(10 \mathrm{~mm}) \\
\text { screening } \\
\text { scans of } 76 \text { malig- } \\
\text { nant and } 413 \text { benign } \\
\text { nodules }\end{array}$ & $\begin{array}{l}\text { AUC of } 0.88 \text { in a leave-one- } \\
\text { out test }\end{array}$ \\
\hline $\begin{array}{l}\text { Iwano et } \\
\text { al. [336] }\end{array}$ & $\begin{array}{l}\text { To classify } \\
\text { nodules into } \\
\text { benign or } \\
\text { malignat }\end{array}$ & $\begin{array}{l}\text { LDA based on } \\
\text { nodule's circu- } \\
\text { larity and second } \\
\text { moment features }\end{array}$ & HRCT (0.5-1 mm). & \\
\hline $\begin{array}{l}\text { Way et } \\
\text { al. [327] }\end{array}$ & $\begin{array}{l}\text { To classify } \\
\text { nodules into } \\
\text { benign or } \\
\text { malignat }\end{array}$ & $\begin{array}{l}\text { LDA or SVM } \\
\text { with stepwise } \\
\text { feature selection }\end{array}$ & $\begin{array}{l}\text { CT scans of } 124 \text { ma- } \\
\text { lignant and } 132 \text { be- } \\
\text { nign nodules in } 152 \\
\text { patients }\end{array}$ & $\begin{array}{l}\text { AUC of } 0.857 \text { in a leave- } \\
\text { one-out test }\end{array}$ \\
\hline $\begin{array}{l}\text { Chen et } \\
\text { al. [333] }\end{array}$ & $\begin{array}{l}\text { To classify } \\
\text { nodules into } \\
\text { benign or } \\
\text { malignat }\end{array}$ & ANN ensemble & $\begin{array}{l}\text { CT (slice thickness } \\
\text { of } 2.5 \text { or } 5 \mathrm{~mm} \text { ) scans } \\
\text { of } 19 \text { malignant and } \\
13 \text { benign nodules }\end{array}$ & $\begin{array}{l}\text { AUC of } 0.915 \text { in a leave- } \\
\text { one-out test }\end{array}$ \\
\hline $\begin{array}{l}\text { Lee et } \\
\text { al. }[338]\end{array}$ & $\begin{array}{l}\text { To classify } \\
\text { nodules into } \\
\text { benign or } \\
\text { malignat }\end{array}$ & $\begin{array}{l}\text { GA-based feature } \\
\text { selection and a } \\
\text { random subspace } \\
\text { method }\end{array}$ & $\begin{array}{l}\text { Thick-slice }(5 \mathrm{~mm}) \\
\text { CT scans of } 62 \mathrm{ma}- \\
\text { lignant and } 63 \text { benign } \\
\text { nodules }\end{array}$ & $\begin{array}{l}\text { AUC value of } 0.889 \text { in a } \\
\text { leave-one-out test }\end{array}$ \\
\hline
\end{tabular}

Matsukoa et al. [337] analyzed the differences in nodule appearance on HRCT images from emphysematous and non-emphysematous patients based on subjective and quantitative measures of nodule appearance. Using a series of 41 emphysematous patients (21 malignant nodules, 20 benign nodules) and 40 non-emphysematous patients (20 malignant nodules, 20 benign nodules), two radiologists, who were blinded to the diagnosis, independently evaluated the appearance of the nodules and classified nodules as being either malignant or benign. The fractal dimensions of the nodule interfaces and circularity of the nodule shape were calculated and the percentage of the nodule surrounded by emphysema was obtained. In patients with emphysema, there were no significant differences in fractal dimension, circularity, spiculation, or frequency of lobulation between malignant and benign nodules. Of all the nodules found in patients with emphysema, 63\% were correctly diagnosed. Thirteen benign nodules $(65 \%)$ were misdiagnosed as malignant in patients with emphysema. Of the nodules in non-emphysematous lungs, 93\% were correctly diagnosed. The mean percentage of emphysematous tissue around the nodule was greater for 
misdiagnosed nodules than for correctly diagnosed nodules $(p<0.003)$, indicating that its presence complicates the diagnosis of pulmonary nodules. Lee et al. [338] developed a two-step supervised learning scheme based on a set of image-based grey level, texture, and shape features combining a genetic algorithm with a random subspace method. They achieved an AUC value of 0.889 in classification between 62 malignant and 63 benign nodules.

Thus, various approaches have been proposed in CADx schemes. Database size varied in different studies; CT scans in the databases included screening LDCT, standard diagnostic CT, and HRCT. Studies on the development of CADx schemes for distinction between malignant and benign lung nodules in CT based on shape and appearance features are summarized in Table 8.

\section{PET/CT Nodule Diagnosis}

Since the combination of PET and CT information has shown an improvement in the delineation of lung nodule contours and estimation of their volumes (see section II.D.3), PET/CT fusion has been widely considered in lung cancer applications such as the tumor staging and pulmonary nodules diagnostics. In PET images, the malignant cells have unregulated metabolism that results in having higher FDG uptake that permits malignancy to be detected. Reported studies [339-346] used this characteristic to detect malignant solitary pulmonary nodules (SPNs) in PET. Solitary pulmonary nodules (SPNs) are single, spherical, well-circumscribed, radiographic opacity that measures $\leq 3 \mathrm{~cm}$ in diameter. Provided a visually validated diagnostics of the SPNs in PET images, these studies [339-346] have reported an SPN diagnostic accuracy with a sensitivity of $88-96 \%$ and a specificity of 70-90\% for malignant cells (see Table 9 for more detail).

Using PET alone without incorporation of CT was reported to provide imprecise information on the exact location of focal abnormalities [347] and can result in false negative errors for lesions with low 18F-FDG uptake value [339, 348, 349] and false positive 
TABLE 9: Evaluation of nodule malignancy in PET

\begin{tabular}{|c|c|c|c|c|}
\hline Study & cases & ACC/PPV & sensitivity & specificity \\
\hline Dewan et al. [342] & 30 & $\mathrm{PPV}=90 \%$ & $95 \%$ & $80 \%$ \\
Gupta et al. [340] & 61 & $\mathrm{PPV}=92 \%$ & $93 \%$ & $88 \%$ \\
Lowe et al. [339] & 89 & $\mathrm{ACC}=91 \%$ & $92 \%$ & $90 \%$ \\
Lee et al. [341] & 71 & $\mathrm{PPV}=86 \%$ & $95 \%$ & $82 \%$ \\
Herder et al. [343] & 36 & $\mathrm{PPV}=72 \%$ & $93 \%$ & $77 \%$ \\
Halley et al. [344] & 28 & $\mathrm{NA}$ & $94 \%$ & $89 \%$ \\
\hline
\end{tabular}

* Accu denotes accuracy and PPV denotes positive productive value

errors in patients with active tuberculosis, histoplasmosis, and rheumatoid nodules. Annema [350] reported the false positive findings of PET to be up to $39 \%$, despite the high negative predictive value of PET, suggesting that the PET positive mediastinal lymph nodes (MLN) were further biopsied in order to confirm or rule out metastasis.

To investigate the integrationof PET and CT information on the accuracy of the malignancy detection, Nie et al. [351] developed an artificial neural networks (ANNs) based on CT alone, PET alone, and both CT and PET for distinguishing benign and malignant pulmonary nodules. Their results show that the accuracy of PET/CT $(\mathrm{Az}=0.95)$ is higher than the CT $(\mathrm{Az}=0.83)$ and PET $(\mathrm{Az}=0.91)$. Nakamoto et al. [352] compared the diagnosis accuracy of CT, side-by-side PET/CT, and software fused PET/CT. They documented that the software fusion of PET/CT resulted in the highest accuracy on patients with lung cancer. Keidar et al. [353] compared the diagnosis performance of PET/CT and PET alone. Using PET-alone resulted in a higher false positive error rate. A higher specificity was achieved using PET/CT suggesting that the anatomical information on CT is an independent crucial variable in determining malignancy. Yi et al. [354] investigated the sensitivity, specificity, and accuracy for predicting malignant nodules on helical dynamic CT and PET/CT. They documented that all malignant nodules were interpreted correctly using dynamic helical 
CT or PET/CT. Lardinois et al. [347] investigated tumor staging using PET/CT vs. PET or CT alone. Their results showed that the PET/CT fusion is a trustworthy means of nodule diagnosis that has improved the accuracy of the tumor staging.

Table 10 summarizes PET/CT results. The experiments involved in these studies [347, 351-354] have shown that using PET/CT achieved a higher diagnostic power than $\mathrm{CT}$ or PET alone, suggesting that the PET/CT fusion may present an advance in lung cancer applications.

TABLE 10: Evaluation of nodule malignancy in fused PET/CT

\begin{tabular}{|c|c|c|c|c|}
\hline Study & cases & ACC/PPV & sensitivity & specificity \\
\hline Keidar et al. [353] & 42 & PPV $=89 \%$ & $96 \%$ & $82 \%$ \\
Yi et al. [354] & 119 & ACC $=93 \%$ & $96 \%$ & $88 \%$ \\
Nie et al. [351] & 92 & ACC $=95 \%$ & NA & NA \\
Nakamoto et al. [352] & 53 & ACC $=87 \%$ & $94 \%$ & $75 \%$ \\
\hline \multicolumn{2}{|c|}{ ACC denotes accuracy and PPV denotes positive productive value }
\end{tabular}

\section{F. Summary and Discussion}

Designing efficient CAD systems for lung cancer is very important since early diagnosis can improve the effectiveness of treatment and increase the patientfs survival rate. In this chapter, an overview of more than 360 articles that appeared in the field are presented to address the challenges and methodologies of the current CAD systems for lung cancer. this chapter addresses the current approaches and their strengths and limitations, which were developed for each stage of lung cancer CAD systems, that is, for lung segmentation, lung nodule detection and segmentation, and lung nodule diagnosis. The work presented in this chapter has been published in the International Journal of Biomedical Imaging (IJBI) [10]. In the final section, this work has been summarized by outlining the research challenges that face each stage in lung cancer CAD systems. 


\section{Research Challenges.}

Several challenges and aspects have been facing CAD systems for lung cancer. These challenges can be summarized as follows:

- To efficiently reduce the search space for lung nodules, accurate segmentation of the lung fields should be provided. The segmentation of lungs is challenging due to inhomogeneities in the lung region and pulmonary structures and of similar densities such as arteries, veins, bronchi, and bronchioles. Technical issues of the lung segmentation techniques should be further investigated. ese technical issues include the automation level of the technique, the sensitivity of the method to the scanning parameters, the efficiency of an algorithm to work with different image modalities (e.g., CT, LDCT, or CE-CT), and the ability of the algorithm to provide a proper lung segmentation in cases with severe pathologies that are associated with inhomogeneities in the pathological lungs.

- Designing an efficient CADe system for detecting lung nodules is still challenging. Important factors should be investigated including the automation level, the speed, the ability to detect nodules of different shapes, for example, irregularly shape nodules not only the spherical ones, and the ability of the CADe system to detect cavity nodules, nodules attached to the lung borders, and small nodules (e.g., less than 3 $\mathrm{mm})$.

- Several challenges and aspects have been facing lung nodule segmentation techniques, such as the ability of a technique to segment the challenging types of nodules, and the automation level of the technique and its robustness.

- Volumetric measurements of growth rate should take into account the global motion of the patients due to their movements and the local motions of the whole lung tissues due to breathing and heart beating. e application of global and local registra- 
tion directly to the segmented nodule leads to the inability to discriminate between the changes due to the true growth of the lung nodules and the changes in the nodule shape which come from breathing and heart beating. These challenging factors should be further investigated.

- Special types of lung nodules such as cavities and ground glass nodules can not be diagnosed using the current growth rate techniques, so further methods and nodule descriptors are needed for diagnosing these nodules.

- The existing set of shape and appearance features (e.g., curvature, and roundness) depend on the accuracy of the nodule segmentation algorithm. is makes a classification method, based on these features, difficult for clinical practitioners to use. So, there is a need for developing qualitative measures that have the ability to describe the whole shape and appearance of the detected nodules.

- Larger databases for efficient validation of the proposed approaches should be provided.

The work presented in this chapter has been published in the International Journal of Biomedical Imaging [10]. 


\section{CHAPTER III \\ SEGMENTATION OF PATHOLOGICAL LUNGS USING ADAPTIVE SHAPE GUIDED BY CT IMAGES APPEARANCE}

This chapter introduces an automated framework for the segmentation of pathological lungs from computed tomography (CT) images. The proposed segmentation methodology is based on a novel 3D joint Markov-Gibbs random field (MGRF) image model of the chest CT images. The proposed model integrates three image features: $(i)$ a novel adaptive shape model of the lung guided by the appearance of both chest and lung tissues from CT images; (ii) first-order appearance model to specify the intensity distribution of the lung and chest for both the original CT images (to specify the local features), and the Gaussian Scale Space (GSS) filtered data (to specify global features), which describes the empirical distribution of image signals using a linear combination of discrete Gaussians (LCDG) with positive and negative components; (iii) second-order appearance model specifies the spatial interaction between the CT image signals using a pairwise MGRF spatial model for both the original and GSS filtered data. The prosed lung segmentation framework has been texted on multiple CT data sets that were acquired using different scanners and acquisition protocols and contains different types of pathologies. Particularly, it has been tested on locally-acquired data (30 data sets) as well as on three publically availably CT databases, namely 55 data sets from the LOLA11 MICCAI challenge, 60 data sets from the EMPIRE10 MICCAI challenge, and 20 data sets from the VESSEL12 ISBI challenge. Segmentation accuracy, for EMPIRE, VESSEL, and the locally-acquired data sets, has been evaluated using the Dice similarity coefficient (DSC), the 95-percentile bidirectional Hausdorff distance (BHD), and absolute lung volume difference (ALVD). The accuracy on the LOLA11 data sets has been blindly evaluated by the challenge organizers. The proposed 
approach has been ranked first over all state-of-the art techniques on the LOLA11 data sets with an average overlap of 98\%, computed over all 55 scans. Quantitative assessment confirmed high accuracy of the proposed segmentation framework and documented the ability to extract pathological lung from CT images.

\section{A. Introduction}

Automated lung segmentation on chest CT scans is crucial for efficient computeraided diagnosis (CAD) of pulmonary diseases, such as, lung cancer. Unfortunately, the large variety of images and diagnostic measurements for different lung pathologies make accurate, fast, and low-cost segmentation a challenge. For instance, pathology-related segmentation failures lose important data, being needed for studying radiation pneumonitis development [355]. As shown by Armato et al. [356] the accuracy of lung segmentation can affect lung nodules detection by $17 \%$.

In the application to CT chest images, most of the earlier segmentation techniques [32, $35,47,52,65-67]$ presume only the lungs are darker than the other chest tissues, which might result in failure to detect nodules in the case of severe lung pathologies. To avoid such failures, more recent lung segmentation methods, which will be briefly reviewed in this section, consider visual appearances [1,357], shapes [55,358-362], or hybrid techniques $[60,62,363-365]$ to account for normal and pathological tissues. A more detailed survey of current lung segmentation techniques can be found in [10].

Appearance-based segmentation employs texture information to distinguish between objects where the edges are not defined by clear boundaries. Wang et al. [357] used classical Haralick's texture descriptors to discriminate, on chest CT scans, between normal and pathological tissues with unclear boundaries for moderate or severe interstage lung disease (ILD). The descriptors, including energy, entropy, contrast, maximum probability, and inverse difference moment, characterize the local gray-level co-occurrence matrices. First, normal tissues and moderate ILD parenchyma were segmented by simple threshold- 


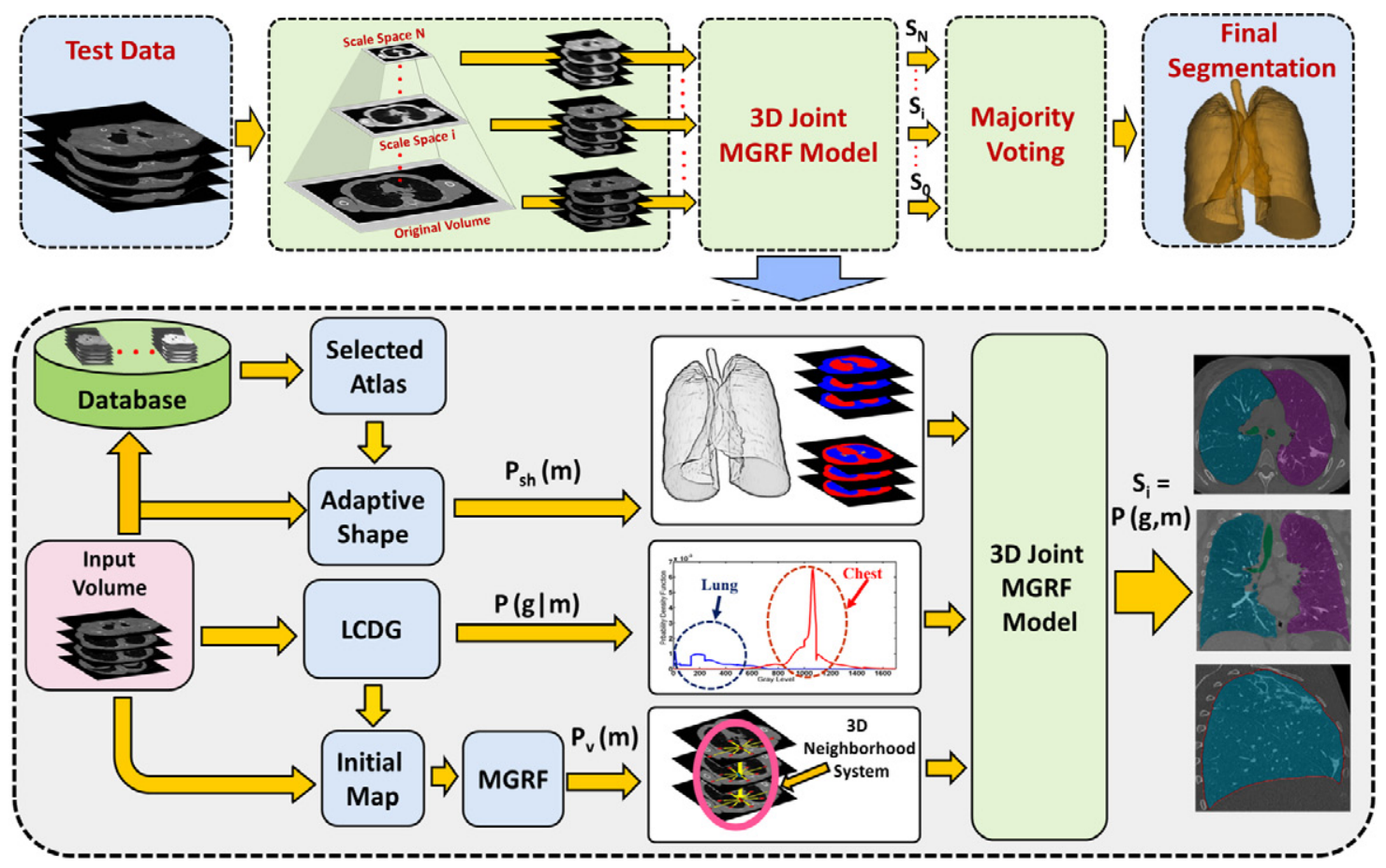

FIGURE 7: The proposed framework for segmenting lungs from chest CT images.

ing of the voxel-wise signals. Then, the abnormal regions with severe ILD, which depict the local descriptors, were found on the appearance images, and combined with the initially segmented regions. This method demonstrated an average overlap of $96.7 \%$ with the "gold standard" manual method on a test database of 76 CT scans (31 normal and 45 abnormal lungs). In another study, Korfiatis et al. [363] segmented lung data, including cases with interstitial pneumonia, first by using voxel-wise gray levels only. Subsequently the initial segmentation was refined by classifying the voxels by a support vector machine classifier to account for statistics of both the gray levels and wavelet coefficients, whereas Lassen et al. [364] used a sequence of morphological operations to refine the initial threshold-based segmentation of the pulmonary airspaces. Kockelkorn et al. [60] segmented the lungs with a $k$-nearest-neighbor classifier, trained on available prior data; however, to account for severe abnormalities, the user had to interactively correct the initial classification.

Shape-based segmentation exploits specific lung shape priors with some variabil- 
ity among the scans to yield more accurate segmentation. Sun et al. [55] matched a 3D active shape model to a CT chest scan to roughly define the initial lung borders and then refined the segmented lungs with a global surface optimization method developed by Li et al. [366]. Van Rikxoort et al. [361] segmented the lungs using region growing and morphological operations methods, then automatically performed a multi-atlas-based refinement if a pathology-related segmentation error was detected automatically due to a statistical deviation from a predefined range of lung volume and shape measurements. Sluimer et al. [360] used 15 chest CT scans to build a probabilistic atlas of normal lung fields and registered a pathological lung scan to the atlas in order to segment the lungs with severe pathologies. To segment pathological lungs, Sofka et al. [59] used a set of anatomical landmarks on the ribs and spine to initialize the shape model and then refined the model by iterative surface deformation. Similarly, an atlas-based segmentation model in combination with a deformable model was applied by Zhou et al. [359] to segment lungs with large tumors; whereas, Nakagomi et al. [362] implemented a graph-cut segmentation technique, that incorporated shape and other prior information about neighboring lung structures, on pathological lung images including pleural effusion.

Hybrid segmentation combines various segmentation techniques to reach higher accuracies. Birkbeck et al. [365] added statistical learning to anatomical constraints derived from neighboring anatomical structures (heart, liver, spleen, and ribs), to segment lungs. These structures were first detected by statistical classifiers and then used as geometrical constraints for deforming the lung mesh. Mansoor et al. [1] segmented pathological lungs with a wide range of abnormalities in two steps. Initially, the lung parenchyma was segmented on the basis of fuzzy connectedness [367], and the differences between the rib-cage and lung parenchyma volumes were analyzed to determine the pathology. Subsequently, the segmentation was refined, by applying texture descriptors, to identify the pathological tissues that might have been missed during the initial segmentation procedure. Kockelkorn et al. [358] divided the chest CT scan into 3D volumes-of-interest (VOIs) with voxels of 
similar intensities and classified each VOI as either the lung or background. Then, the misclassified voxels were corrected either interactively or using a slice-wise supervised classification technique. Hua et al. [62] segmented the lungs by optimizing a graph-supported cost function of voxel-wise intensities and their spatial gradients, taking into account the boundary smoothness and rib constraints.

However, the above methods for automated or semi-automated segmentation of normal and pathological lungs from CT chest images have several drawbacks. Specifically, some of the methods rely on expensive and user-dependent interactions with a radiologist, or depend on anatomical landmarks, which are difficult to determine, especially when pathological tissues are present. Furthermore, when a pathology detector is employed, the overall accuracy considerably decreases after the detection fails. In addition, active appearance models make segmentation too sensitive to model initialization and number of control points. Moreover, most of the methods were designed for a specific type of lung pathology, e.g., nodules, and thus may fail on other types of pathological tissues. To partially overcome these drawbacks, a novel framework has been proposed, being capable to segment with a high level of accuracy both normal and pathological lungs from chest CT images acquired via various scanners and protocols. Due to integrating an adaptive shape prior with easy-to-learn first-order visual appearance models and a $2^{\text {nd }}$-order 3D MGRFbased model of spatial voxel interactions, the framework is applicable to a wide variety of pathologies, including tumors, pulmonary sarcoidosis, pleural effusion, consolidation, fibrosis, and cavities. To the best of our knowledge, it is the first time to introduce a probabilistic map accounting for not only region labels, but also intensities in neighboring spatial voxel locations as an adaptive shape prior. The framework, detailed in Section III.B, was extensively validated on three databases (Section III.D) and ranked first by the third-party evaluation of LOLA11 challenge. 


\section{B. Methods}

All 3D CT chest scans are initially pre-processed to identify their background voxels, (air and bed), by region growing and analyzing connected components. Since detailed prior information about lung shapes significantly improves the segmentation accuracy, the proposed framework (Figure 7) builds a spatially-variant shape prior from a pre-selected training database of 3D CT scans of normal and pathological lungs. The training scans are accurately co-aligne using a B-spline-based nonrigid registration [368], detailed in Section III.B.1. Unlike more conventional alternatives, the proposed shape prior is adapted to a given input, or test CT scan. The latter is similarly aligned to the training database and a $3 \mathrm{D}$ deformation field of aligning voxel-to-voxel translations is stored in order to guide the process of building the shape prior.

The framework analyzes both the original input and its Gaussian scale-space (GSS) versions, being formed by convolving with 3D moving Gaussian kernels [369] (Section III.B.2). The GSS smoothing adds longer-range properties to original voxel-wise intensities and their pairwise co-occurrences in the nearest 26-neighborhoods of each voxel. For segmenting the input image, first- and second-order lungs-background models are learned and combined with the shape priors separately for the input image and each of its GSS versions. The first-order models are built by accurate LCDG-based approximations of empirical marginal probability distributions of intensities over the whole image and estimations of individual marginal distributions of the lung and background intensities [370] (Section III.B.3). The $2^{\text {nd }}$-order spatially uniform MGRF models with multiple pairwise interactions of the nearest-neighbor region labels [371] are learned to describe initial lungs-background region maps (Section III.B.4).

The input image, as well as each of its smoothed GSS versions, is separately segmented by combining their learned probabilistic first- and $2^{\text {nd }}$-order models together with the adaptive shape priors into a joint MGRF model of images and their region maps (Section III.B.5). Finally, a majority rule is used to merge the resulting separate segmentations 
into a final lung-background region map (Section III.B.6).

Basic notation: Let $\mathbf{Q}=\{0,1, \ldots, Q-1\}$ and $\mathbf{K}=\{0,1\}$ be a finite set of integer gray levels and region labels (background chest tissues - “0”, and lung tissues “1”), respectively. Let $\mathbf{r}=(x, y, z)$ be a voxel in integer 3D $(x, y, z)$-coordinates spanning from $(0,0,0)$ to $(X, Y, Z)$. Let a finite $3 \mathrm{D}$ arithmetic lattice $\mathbf{R}=\{\mathbf{r}=(x, y, z): 0 \leq x \leq$ $X, 0 \leq y \leq Y, 0 \leq z \leq Z\}$ support the grayscale CT images, $\mathbf{g}=\left\{g_{\mathbf{r}}: \mathbf{r} \in \mathbf{R} ; g_{\mathbf{r}} \in \mathbf{Q}\right\}$, and their region, or segmentation maps, $\mathbf{m}=\left\{m_{\mathbf{r}}: \mathbf{r} \in \mathbf{R} ; m_{\mathbf{r}} \in \mathbf{K}\right\}$.

\section{Adaptive probabilistic shape prior}

The proposed appearance-guided adaptive shape prior is built from a pre-selected and manually segmented training database of $3 \mathrm{D}$ chest $\mathrm{CT}$ scans. To reduce variations and maximize overlaps between the lungs for estimating the prior, each source volume $f$ (i.e., each of the database subjects) is aligned to the target volume, or reference template $g$ on a domain $\Omega \subset \mathbb{R}^{3}$ by using a non-rigid registration. Given a certain source $f$, the registration estimates the deformation field $\mathcal{T}$ for all $x \in \Omega$, by displacing a sparse grid, $\Omega^{\prime} \subset \Omega$; $\left(\left|\Omega^{\prime}\right| \ll|\Omega|\right)$, of control points, $\xi$ :

$$
\mathcal{T}(x)=x+\sum_{\xi \in \Omega^{\prime}} \zeta(\|x-\xi\|) \Delta_{\xi}
$$

where $\Delta_{\xi}$ is the displacement vector of the control point $\xi$ and the weighting function $\zeta($. measures the contribution of any control point in $\Omega^{\prime}$ to the displacement of a point in $\Omega$. The goal deformation field minimizes the point-wise dissimilarity between the target $g$ and the deformed source $f$ :

$$
E(\mathcal{T})=\frac{1}{\left|\Omega^{\prime}\right|} \sum_{\xi \in \Omega^{\prime}} \int_{\Omega} \frac{\phi(g(x), f(\mathcal{T}(x)))}{\zeta(\|x-\xi\|)} d x
$$

where $\phi$ is the dissimilarity function (sum of absolute differences has been used). The objective function in Equation (3) is minimized using a Markov random field model of dis-

placements of the control points $\xi$ [372]. The dense displacement field is then determined 
from the control point displacements through representing free form deformations (FFDs) via cubic B-splines. This method has been selected because it is fully automated (no manual initialization or hand-picked landmarks) and has low computational time. More details can be found in $[368,372,373]$.

To handle both the normal and pathological lungs, the used database, contains 3D scans of different normal and pathological lungs, $\mathbf{S}=\left\{\mathbf{s}_{i}=\left(\mathbf{g}_{i}, \mathbf{m}_{i}\right): i=1,2, \ldots, N\right\}$, having been chosen to represent typical inter-subject variations, and their true region maps. The database subjects are selected using the principal component analysis (PCA). The PCA calculates principal components (PCs) of the covariance matrix between all the available subjects from the database (50 subjects in this work). Then, instead of defining a new template domain for the top $N$ PCs, the original $N$ subjects with the highest-magnitude coefficients of each of the top $N$ PCs are selected to approximate projections computed by the PCA [374] for each test image. In the experiments below, the $N=20$ training images, having been selected from the locally acquired database of 50 subjects, had different sizes, data spacing, and slice thickness, which ranged from $512 \times 512 \times 270$ to $512 \times 512 \times$ 450; 0.703 to $1.37 \mathrm{~mm}$, and 0.703 to $2.50 \mathrm{~mm}$, respectively. Co-aligning these images with the reference template overcomes their variations and makes their size and resolution identical. Seeing that every training image has its own deformation field that maps its voxels to the reference template, accurate mappings between the template and training images are guaranteed, shown in Figure 8.

A test 3D CT image $\mathbf{t}$ to be segmented is co-aligned with the training database using the same template and B-spline-based nonrigid registration [368]. Then its customized atlas is extracted by computing conventional normalized cross correlations (NCC) of Equation (4) between the chest region in the aligned test image $\mathbf{t}$ and the chest region in each database image, $\mathbf{g}_{1: i}$, to select the top $J$ similar lungs.

$$
N C C_{\mathbf{t}, \mathbf{g}_{i}}=\frac{\sum_{\mathbf{r} \in \mathbf{R}}\left(t_{\mathbf{r}}-\mu_{\mathbf{t}}\right)\left(g_{i: \mathbf{r}}-\mu_{\mathbf{g}_{1: i}}\right)}{\sqrt{\sum_{\mathbf{r} \in \mathbf{R}}\left(t_{\mathbf{r}}-\mu_{\mathbf{t}}\right)^{2}} \sqrt{\sum_{\mathbf{r} \in \mathbf{R}}\left(g_{i: \mathbf{r}}-\mu_{\mathbf{g}_{i}}\right)^{2}}}
$$


Algorithm 1 The Proposed Lung Segmentation Algorithm

Input: Read the original 3D CT data

\section{○ Appearance-based Adaptive Shape Creation:}

1. Register the test subject to the training database to get the deformation fields for each voxel.

2. Generate the GSS data.

3. Perform a cross correlation similarity between the test subject and each subject in the training database and select the most similar subjects.

4. Estimate the appearance-based shape model $P_{\mathrm{sh}}(\mathbf{m})$ by calculating the value of the shape prior probability at each voxel using the following steps for the original and GSS data:

(a) Transform each voxel in the test subject to the training database domain using the calculated deformation field.

(b) Initialize an $N_{1 i} \times N_{2 i} \times N_{3 i}$ search space centered at the mapped voxel.

(c) Find the voxels within the search space that is close to the voxel's Hounsfield value within $\pm \tau$.

(d) If no corresponding voxels are found, increase the search space size and repeat Step 4-(b, and c) until a correspondence is found or the predefined maximum search space size is reached.

(e) If no correspondences are found, increase the tolerance with a predefined value $\Delta \tau$ and repeat Step 4-(b,c, and d) until a correspondence is found.

(f) Calculate the label probabilities for each voxel based on the relative occurrence of each label in the found voxel's correspondences search results.

$\circ$ Lung Segmentation for the original and GSS data:

1. Find an initial map $\mathbf{m}$ by voxelwise Bayesian MAP classification of a given CT image using the estimated appearance-based shape probabilities.

2. Estimate the conditional intensity model $P(\mathbf{g} \mid \mathbf{m})$ by identifying the LCDG models of signals of each object class represented by one of the dominant modes.

3. Use the initial region map $\mathbf{m}$ to identify the MGRF model $P_{\mathbf{V}}(\mathbf{m})$ of region maps.

4. Perform the final Bayesian segmentation of the lung regions using the joint MGRF model in Equation (11).

- Output: Obtain the final segmentation of the lung regions using majority voting of individual segmentation of the original and GSS data. 
where $\mu_{\mathbf{t}}$ and $\mu_{\mathbf{g}_{i}}$ are the average intensities for $\mathbf{t}$ and $\mathbf{g}_{i}$.

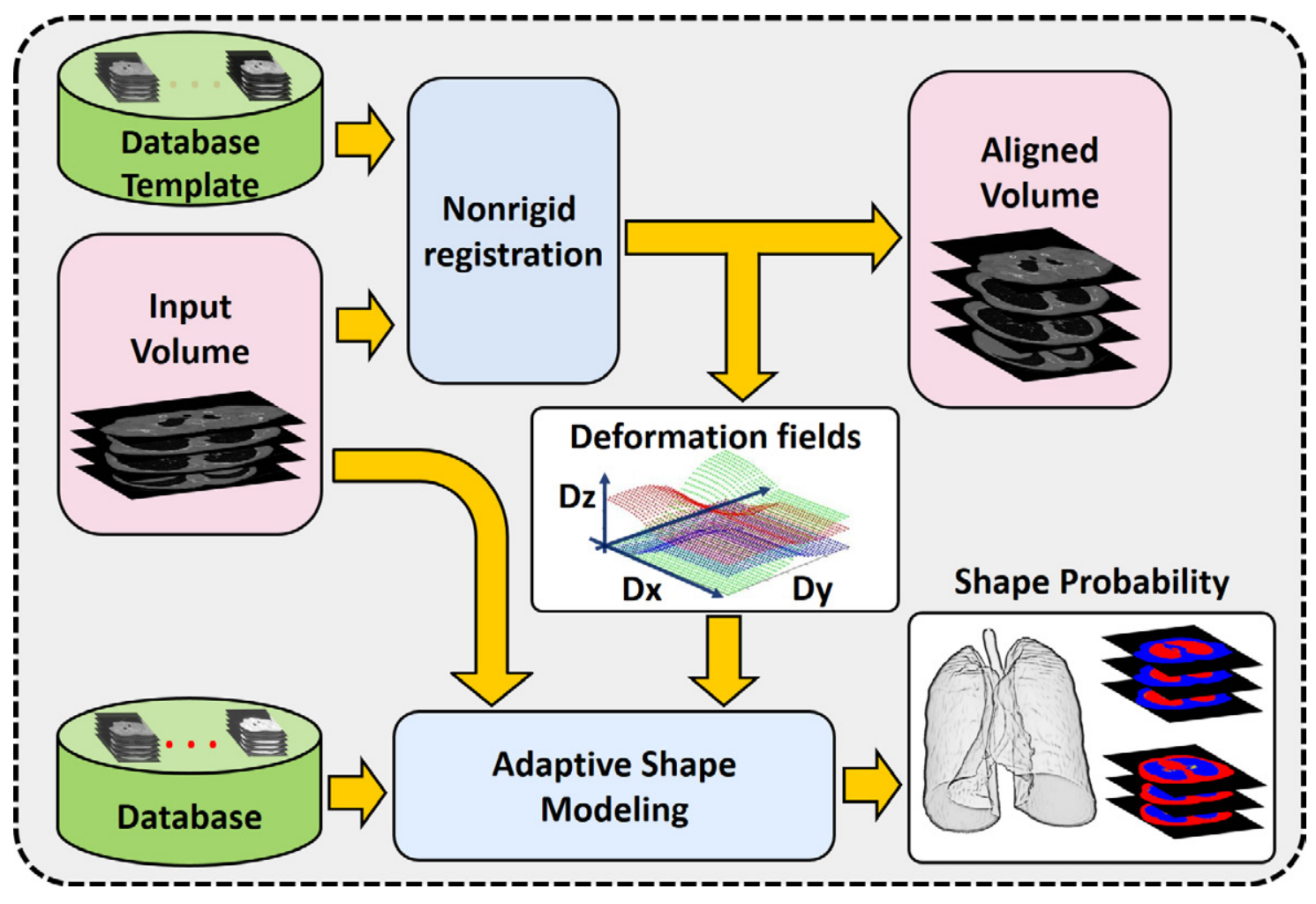

FIGURE 8: Test subject alignment and shape probability calculations.

Visual appearances of the test and training images guide the shape prior adaptation as follows (this process is also outlined in Algorithm 1 summarizing the proposed segmentation framework). Each voxel $\mathbf{r}$ of the test image $\mathbf{t}$ is mapped to the database lattice by the deformation field aligning $\mathbf{t}$ to the database. A subject-specific atlas is built from the top $J ; J \leq N$, training images $\mathbf{g}_{i}$ selected in accord with their correlations $N C C_{\mathbf{t}, \mathbf{g}_{i}}$ of Equation (4) ( $J=15$ in the experiments below). To adapt the shape prior at each mapped location $\mathbf{r}$, an initial search cube $\mathbf{C}_{\mathbf{r}}$ of size $n_{x: \mathrm{i}} \times n_{y: \mathrm{i}} \times n_{z: \mathrm{i}}$ is centered at the mapped location $\mathbf{r}$ for finding in the cube all the atlas voxels with signal deviations to within a predefined fixed range, $\tau$, from the mapped input signal, $t_{\mathbf{r}}$. If such voxels are absent in the atlas, the cube size increases iteratively until the voxels within the predefined signal deviation range are found or the final cube size is reached $\left(n_{x: \mathrm{i}}=n_{y: \mathrm{i}}=n_{z: \mathrm{i}}=3 ; \tau\right.$ from 
50 to 125 with the step of $\Delta_{\tau}=25$, and the final cube size of $n_{x: \mathrm{f}}=n_{y: \mathrm{f}}=n_{z: \mathrm{f}}=11$ were used in the experiments). Then the voxel-wise probabilities, $P_{\mathrm{sh}: \mathrm{r}}(k) ; k \in \mathbf{K}$, for the adaptive shape prior $P_{\mathrm{sh}}(\mathbf{m})=\prod_{\mathbf{r} \in \mathbf{R}} P_{\mathrm{sh}: \mathbf{r}}\left(m_{\mathbf{r}}\right)$ are estimated based on the found voxels of similar appearance and their labels. Let $\boldsymbol{\nu}_{j: \mathbf{r}}=\left\{\boldsymbol{\rho}: \boldsymbol{\rho} \in \mathbf{R} ; \boldsymbol{\rho} \in \mathbf{C}_{\mathbf{r}} ;\left|g_{j: \boldsymbol{\rho}}-t_{\mathbf{r}}\right| \leq \tau\right\}$ be a subset of similar voxels within the cube $\mathbf{C}_{\mathbf{r}}$ in the training image $\mathbf{g}_{j}$. Let $\nu_{j: \mathbf{r}}=\operatorname{card}\left(\boldsymbol{\nu}_{j: \mathbf{r}}\right)$ denote the cardinality (number of voxels) of this subset; $\nu_{\mathbf{r}}=\sum_{j=1}^{J} \nu_{j: \mathbf{r}}$, and $\delta(z)$ be the Kronecker's delta-function: $\delta(0)=1$ and 0 otherwise. Then, the calculated adapted shape probability $P_{\mathrm{sh}: \mathrm{r}}(k)$ for each voxel is calculated by Equation (5) as follow:-

$$
P_{\mathrm{sh}: \mathbf{r}}(k)=\frac{1}{\nu_{\mathbf{r}}} \sum_{j=1}^{J} \sum_{\boldsymbol{\rho} \in \boldsymbol{\nu}_{j: \mathbf{r}}} \delta\left(k-m_{j: \boldsymbol{\rho}}\right)
$$

Figure 9 gives a 2D illustration of how the mapping from the test subject and the searching process occurs to calculate the adaptive shape probability for each voxel in an adaptive way. The search starts with a window centered at the original voxel location in the test subject and all of its mapped locations in the database (blue square), searching for intensity values within $\pm \tau$ in this window and increase both $\tau$ and the window size (dotted green square) till finds a contributing voxels. Consequently, the founded voxels' labels contribute to that voxel probability calculations as given in Equation (5).

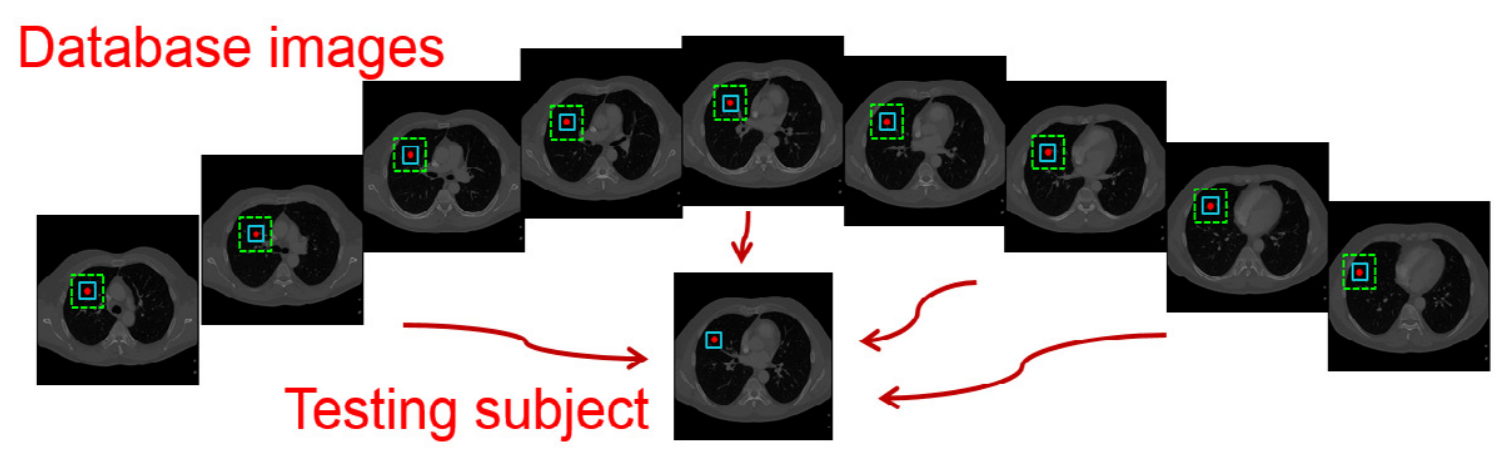

FIGURE 9: A typical 2D Illustration of how the shape probability for each voxel calculated in an adaptive way in terms of spatial locations based on varying window size and in terms of intensity value based on changing tolerance. 
The complete algorithm for adaptive shape prior is illustrated in Algorithm 2 and

\section{illustrative output is shown in Figure 10.}

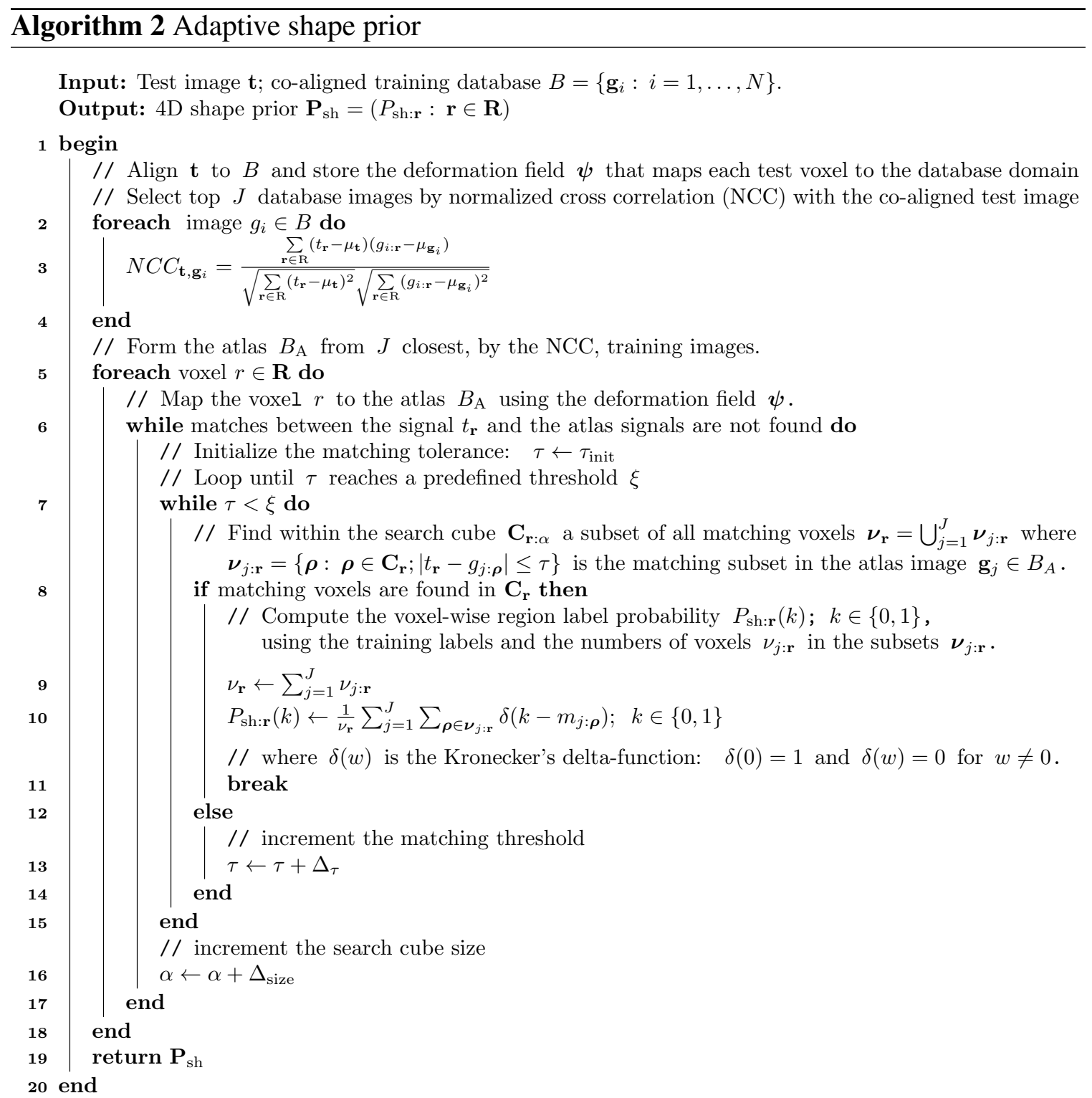




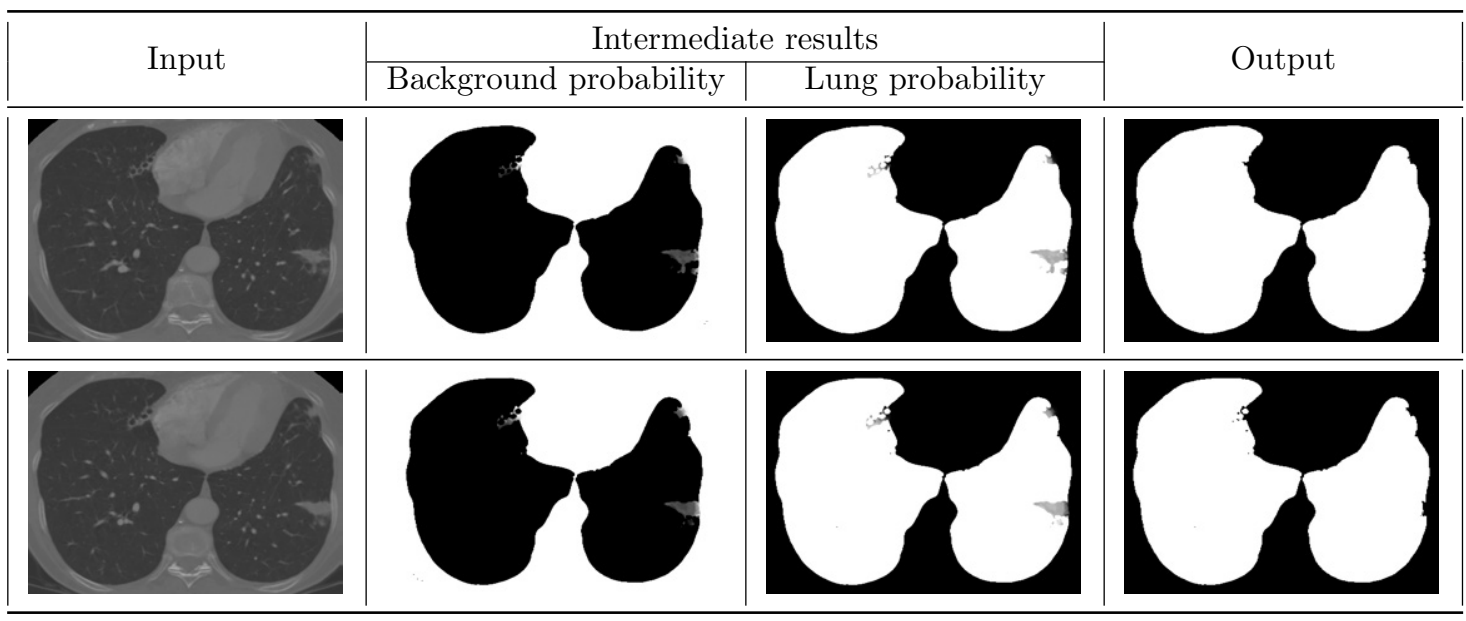

FIGURE 10: Axial planar sections of the 3D CT images; background / lung shape probabilities, and the final segmentation maps.

2. Gaussian scale space (GSS) smoothing

This operation $\mathbf{g}_{\sigma}=\mathbf{g} * \mathbf{h}_{\sigma}$ convolves a 3D CT image $\mathbf{g}$ with a 3D Gaussian kernel $\mathbf{h}_{\sigma ; \mathbf{c}}=\left(h_{\sigma: \mathbf{r}-\mathbf{c}}:(\mathbf{r}, \mathbf{c}) \in \mathbf{R}^{2}\right)[369]:$

$$
h_{\sigma: \mathbf{r}-\mathbf{c}}=\frac{1}{(2 \pi)^{1.5} \sigma^{3}} \exp \left(-\frac{1}{2 \sigma^{2}}|\mathbf{r}-\mathbf{c}|^{2}\right)
$$

where $|\mathbf{r}-\mathbf{c}|=\sqrt{\left(x-x_{\mathbf{c}}\right)^{2}+\left(y-y_{\mathbf{c}}\right)^{2}+\left(z-z_{\mathbf{c}}\right)^{2}}$ denotes the Cartesian voxel-to-voxel distance and $\mathbf{c}=\left(x_{\mathrm{c}}, y_{\mathrm{c}}, z_{\mathrm{c}}\right)$ is the kernel's center. The GSS smoothing reduces signal noise affecting some chest CT scans, integrates longer-range properties of the scans in each voxel-wise signal, and excludes some distortions that may be caused by pathologies. Figure 11 show three different scales of 3D GKs and their convolution with the original CT images are demonstrated in Figure 12. Because it flattens the original image, i.e., decreases the maximal and increases the minimal intensities, only two versions smoothed with the kernels $9 \times 9 \times 5 ; \sigma^{2}=4.5$, and $17 \times 17 \times 5 ; \sigma^{2}=8.5$; were used in the experiments below to extend the overall description of the CT image without losing too many details and affecting the segmentation accuracy. The complete algorithm for the generation of 
Gaussian scale space smoothing is illustrated in Algorithm 3 and an illustrative output is shown in Figure 13.

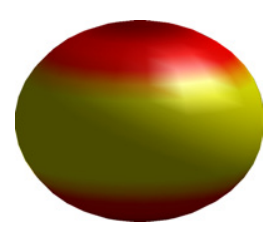

(a)

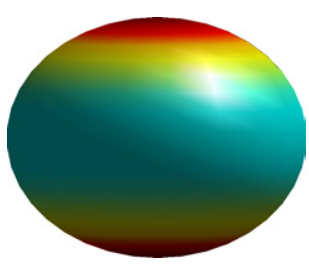

(b)

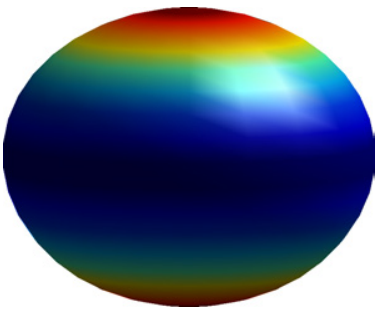

(c)

FIGURE 11: Three different scales of the 3D Gaussian kernels (GK).

(A)

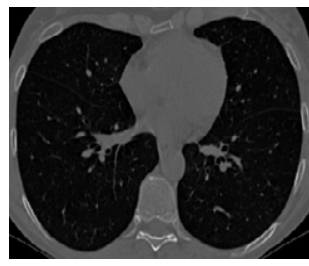

(S)

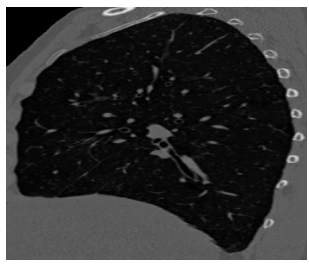

(C)

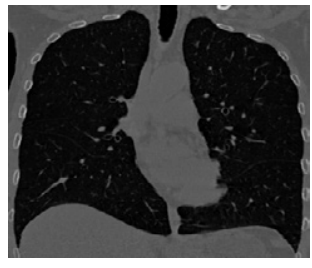

(a)
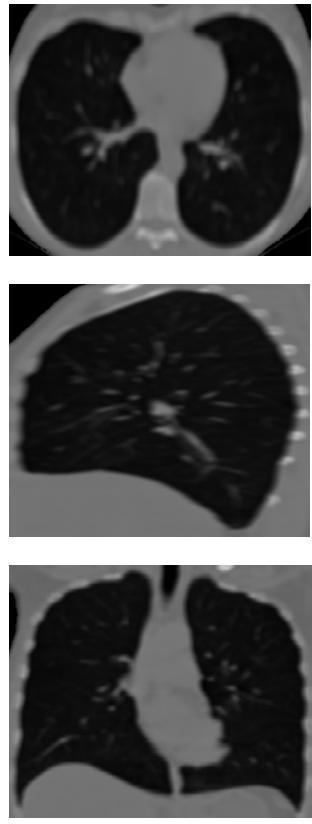

(b)
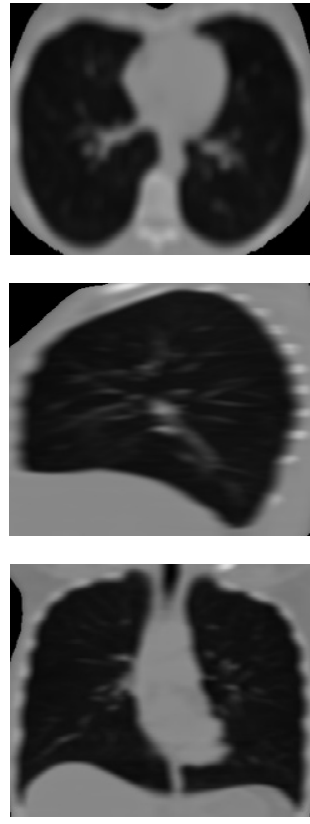

(c)
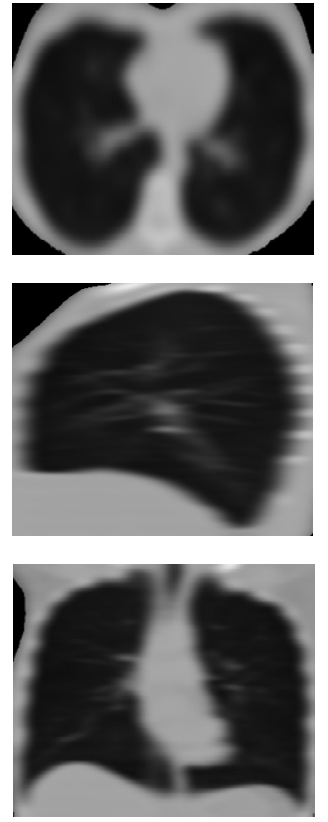

(d)

FIGURE 12: 2D projection of the original and GSS filtered CT images on the axial (A), sagittal (S), and coronal (C) planes using 3D GKs shown in Figure 11. Original CT images(a); and the smoothed CT images (b,c,d) using the 3D GKs in Figure 11(a), Figure 11(b); and Figure 11(c), respectively. 


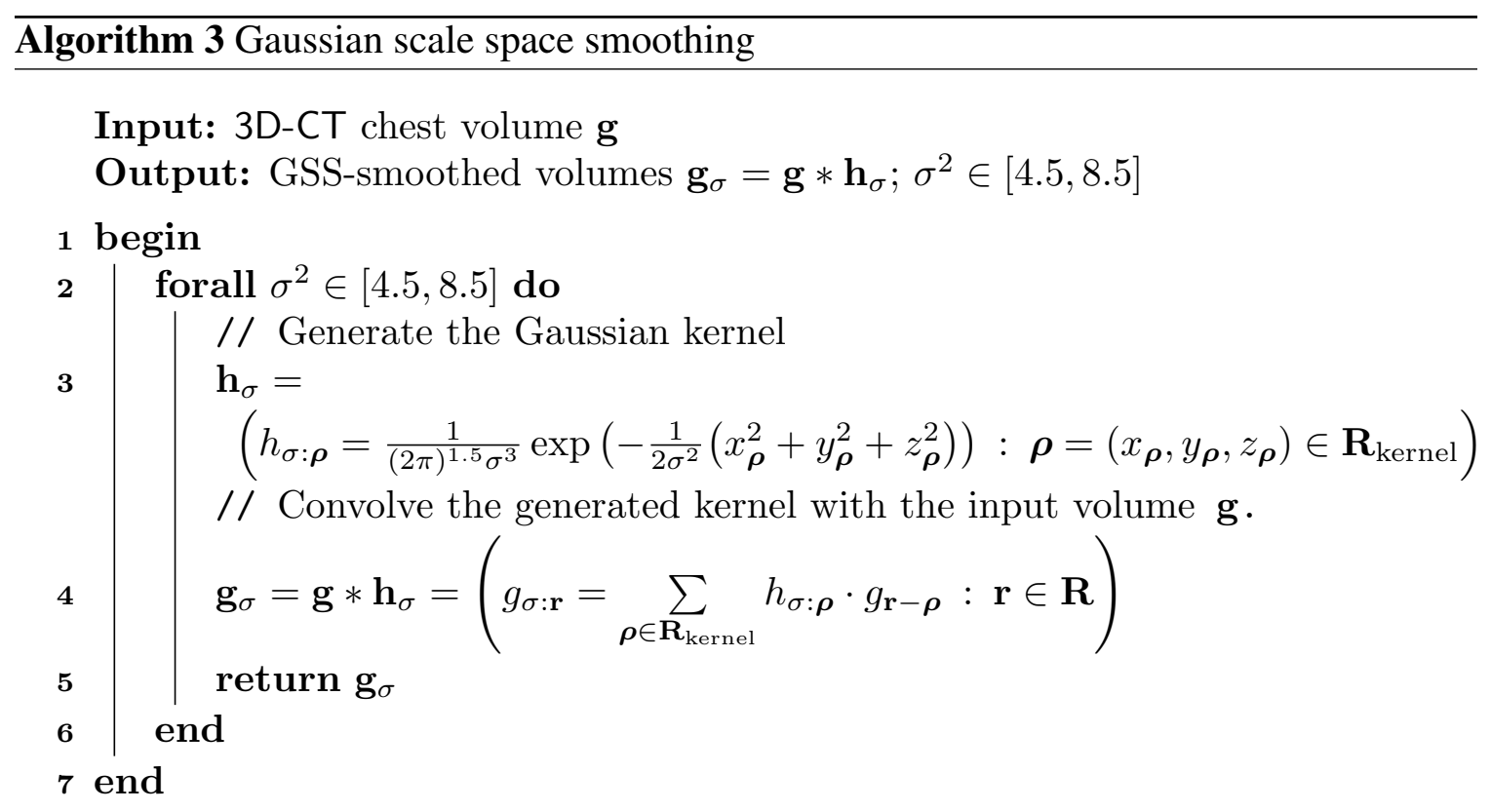

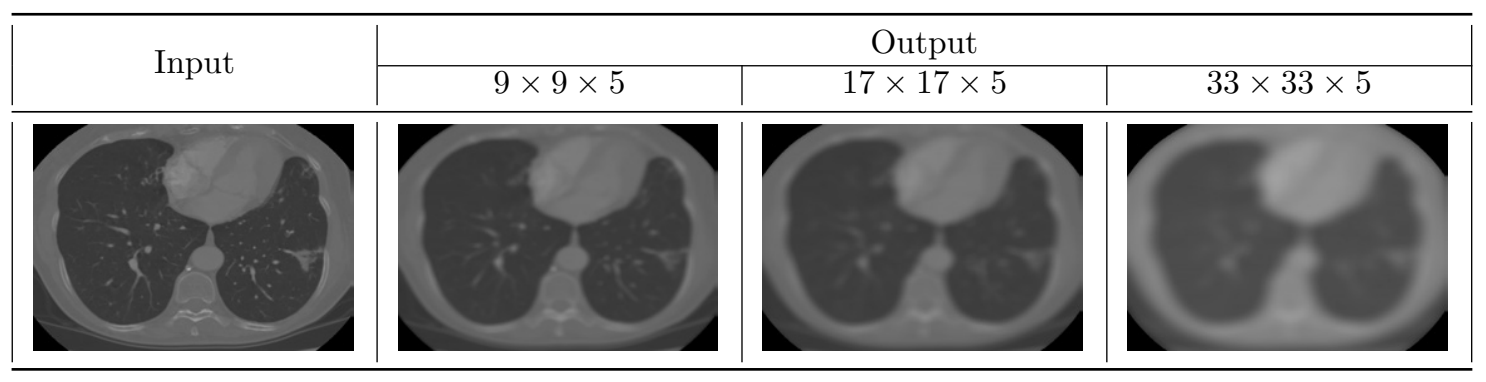

FIGURE 13: Axial planar sections of the original 3D CT image and its GSS-smoothed versions obtained with the $9 \times 9 \times 5,17 \times 17 \times 5$, and $33 \times 33 \times 5$ kernels.

3. First-order appearance (intensity) modeling

To accurately separate and approximate conditional marginal probability distributions of intensities (Hounsfield units) for the lungs and surrounding chest tissues, the CT image is modeled as a simple independent random field (IRF) with the same mixed distribution of intensities at each voxel. The mixed empirical distribution of the voxel-wise intensities over the whole image is approximated with an LCDG with two dominant posi- 
tive DGs and multiple sign-alternate (positive and negative) subordinate DGs. The number of the subordinate DGs and parameters (means and variances) of all the DGs are estimated with the classical Expectation-Maximization (EM) algorithm and its modification accounting for the alternate signs of the DGs [375]. Then the obtained LCDG is separated into conditional lung and chest intensity models for defining the conditional IRF of image signals, given a region map:

$$
P(\mathbf{g} \mid \mathbf{m})=\prod_{\mathbf{r} \in \mathbf{R}} p_{m_{\mathbf{r}}}\left(g_{\mathbf{r}}\right)
$$

Basic steps of estimating the conditional lung and chest LCDG models, $P_{k}=\left(p_{k}(q)\right.$ : $q \in \mathbf{Q}) ; k \in \mathbf{K}=\{0,1\}$, are illustrated in Figure 14, and Figure 15 shows a 3D CT image; its GSS-version for the $33 \times 33 \times 5 \mathrm{kernel}$, and the corresponding final estimated conditional LCDG models for chest and lung marginals. The complete algorithm for firstorder appearance modeling is illustrated in Algorithm 4 and an illustrative output is shown in Figure 17. 


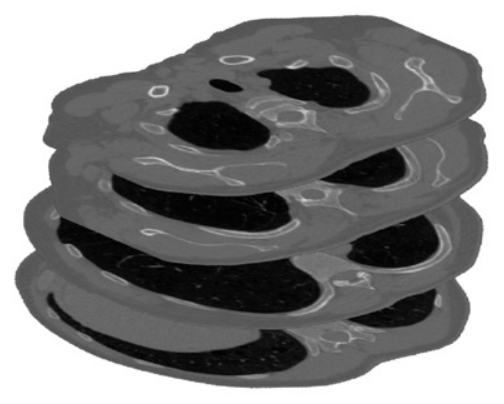

(a)

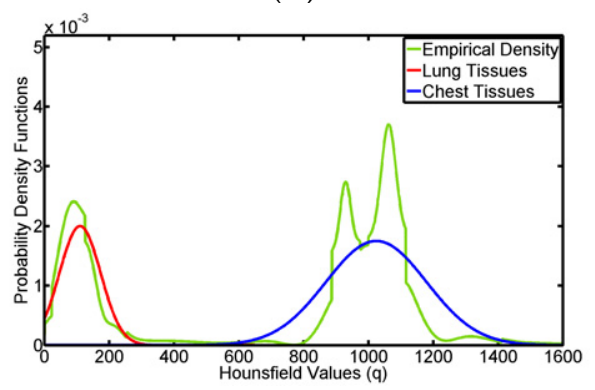

(c)

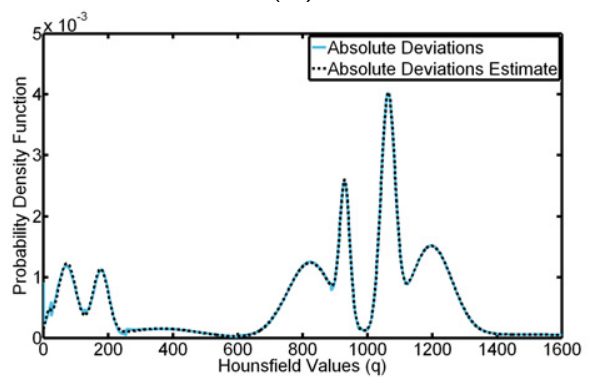

(e)

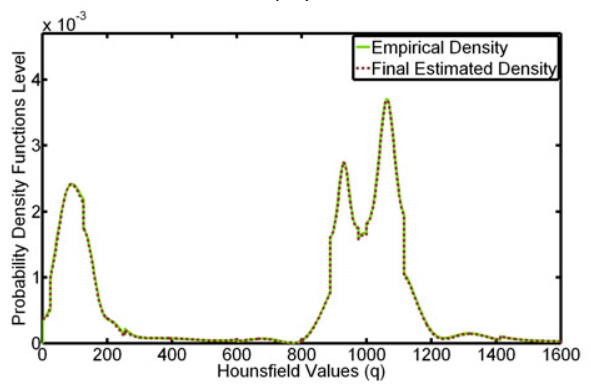

(g)

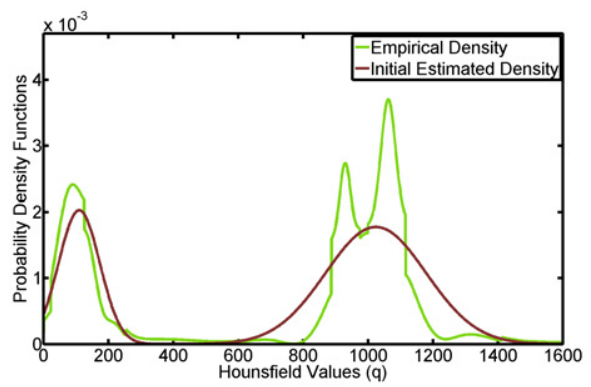

(b)

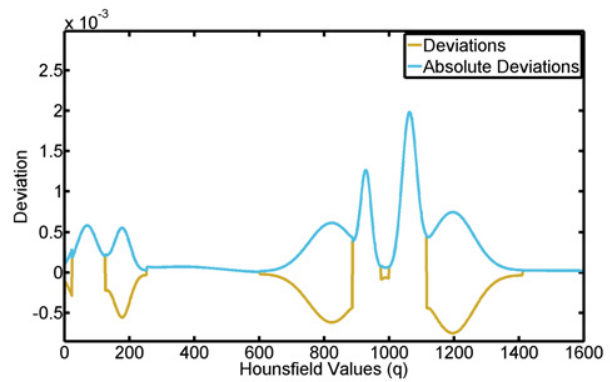

(d)

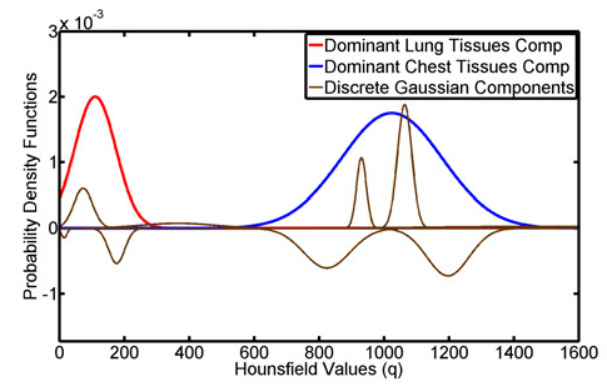

(f)

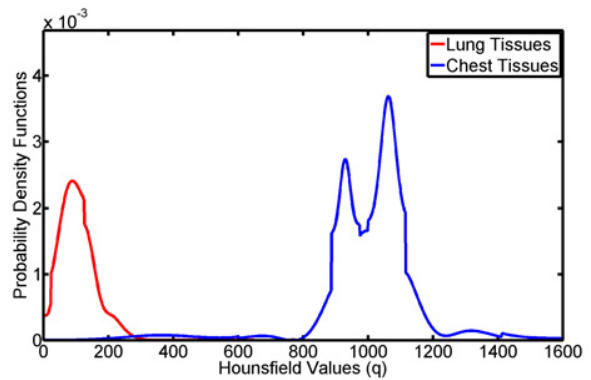

(h)

FIGURE 14: Typical 3D CT chest data (a); and estimated density $(b)$ using only two dominant Gaussian components $(c)$, deviation between empirical and estimated densities $(d)$, estimated density of absolute deviation (e) LCDG components ( $f$ ), final estimated density $(g)$, and final estimated marginal density for each class $(h)$. 


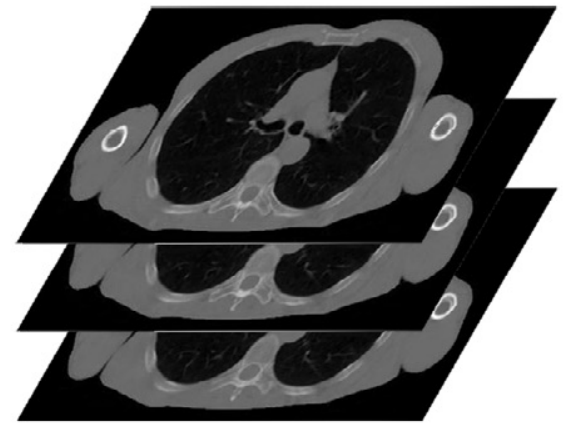

(a)

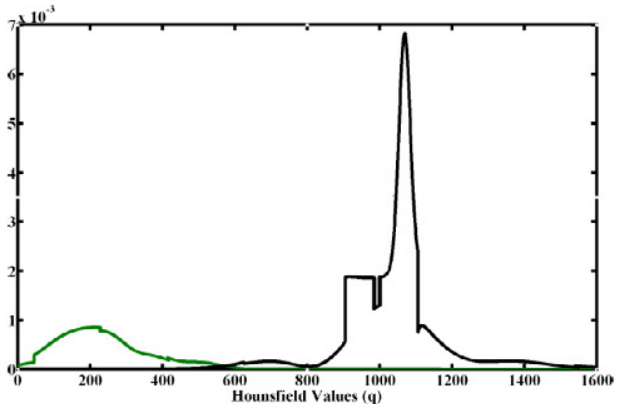

(c)

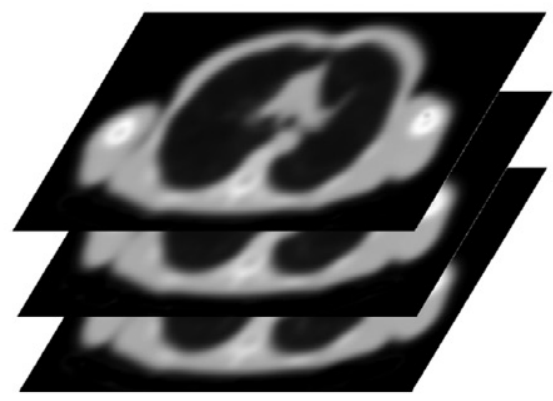

(b)

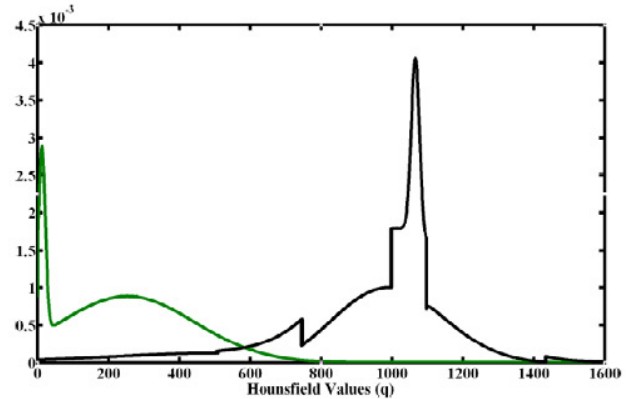

(d)

FIGURE 15: 3D CT image (a) and its GSS-filtered version (b) for the $33 \times 33 \times 5$ kernel, with the final estimated conditional LCDG models for chest and lung marginals (c,d).

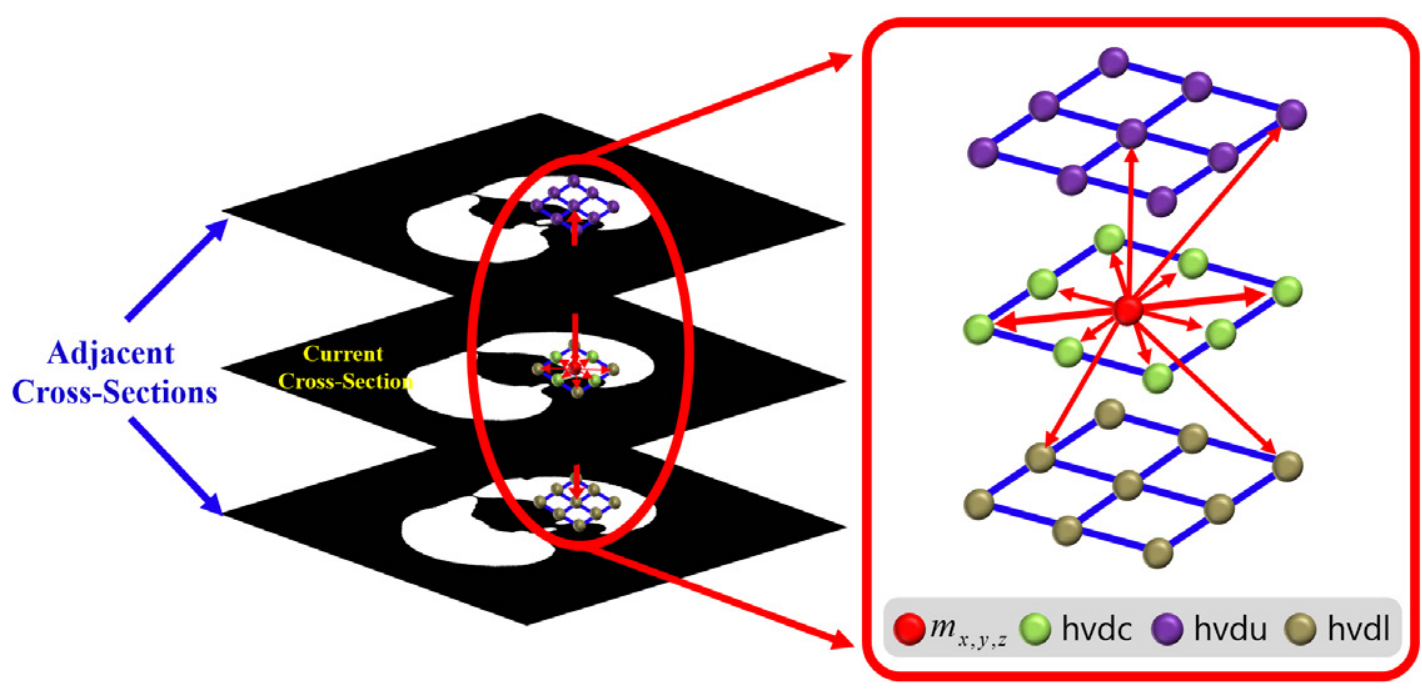

FIGURE 16: The 3D neighborhood system for pairwise MGRF model. 


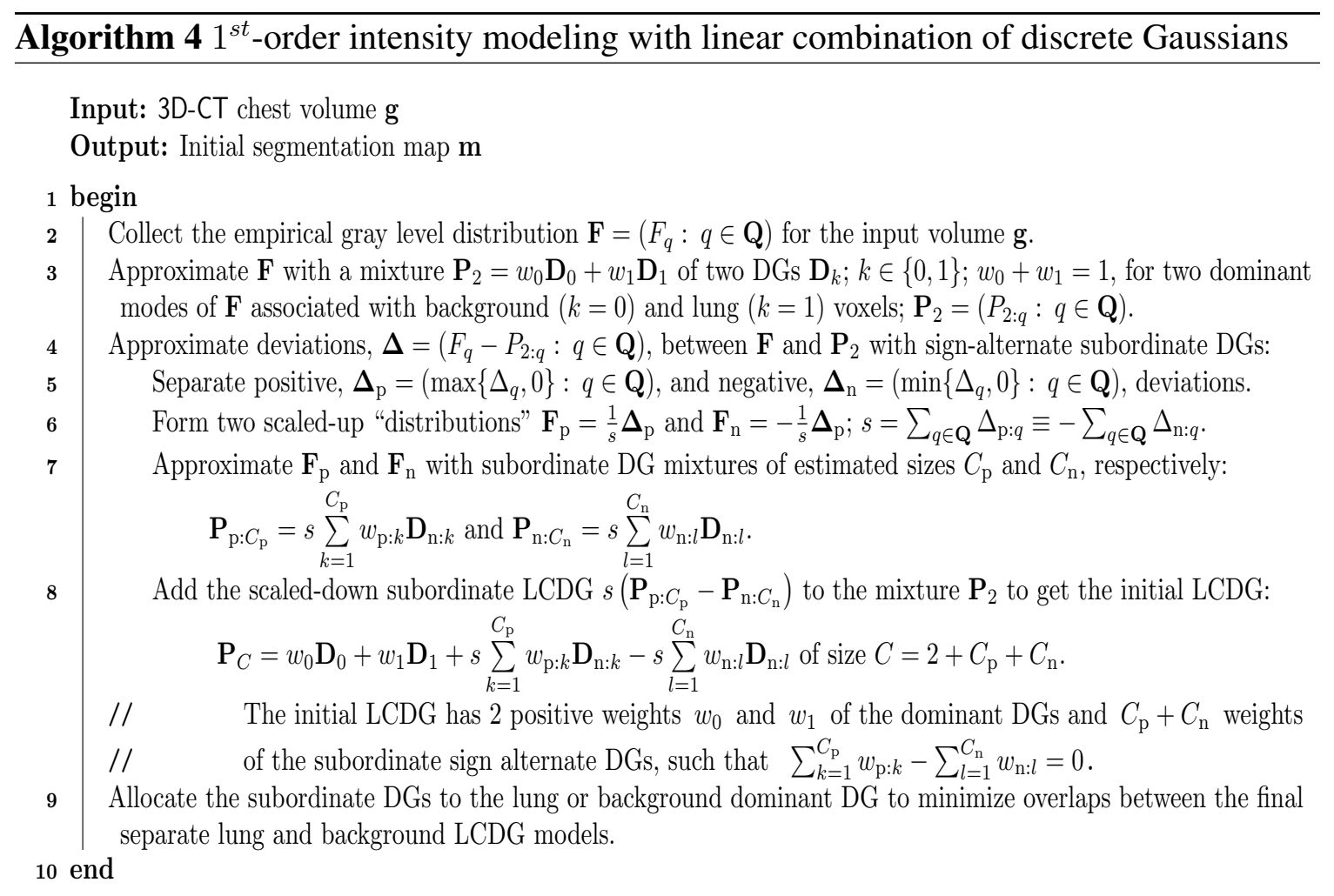

\begin{tabular}{|l|l|l|l|l|}
\hline \multicolumn{3}{|c|}{ Original Image } & \multicolumn{3}{c|}{ GSS smoothing } \\
\hline Input & Output & Input & Output \\
\hline & & & & \\
\hline
\end{tabular}

FIGURE 17: Initial segmentation maps after voxel-wise classification by the LCDG modeling of original and GSS-smoothed images.

4. Modeling pairwise spatial interactions of lung labels

To account for lungs inhomogeneities that may affect the initial Bayesian segmentation, spatial dependencies between the lung voxels are modeled with a spatially invariant second-order MGRF [370,371] with the nearest voxel 26-neighborhood (see Figure 16). 
This modeling enhances the initial segmentation by calculating the likelihood of each voxel to be lung or non-lung on the basis of the initial labeling of the adjacent voxels. By symmetry considerations, the dependencies are uniform, i.e., independent of orientations in the lattice. Let $\mathbf{V}_{a}=\left\{V_{a}\left(k, k^{\prime}\right)=V_{a \text {, eq }}\right.$ if $k=k^{\prime}$ and $V_{a}\left(k, k^{\prime}\right)=V_{a, \text { ne }}$ if $k \neq k^{\prime}$ : $\left.k, k^{\prime} \in \mathbf{K}\right\}$ denote bi-valued Gibbs potentials describing three types of symmetric pairwise dependencies between the labels: $a \in \mathbf{A}=\{$ hvdc, hvdu, hvdl $\}$ where hvdc, hvdu, and hvdl indicate the horizontal-vertical-diagonal dependencies between each voxel and its closest 26-neighbors in the current (c), upper (u), and lower (l) CT slices, respectively, specified by the subsets of inter-voxel coordinate offsets: $\mathbf{N}_{\text {hvdc }}=\{( \pm 1,0,0),(0, \pm 1,0)\}$, $\mathbf{N}_{\text {hvdu }}=\{(0,0,1),( \pm 1, \pm 1,1)\}$, and $\mathbf{N}_{\text {hvdl }}=\{(0,0,-1),( \pm 1, \pm 1,-1)\}$. Then the Gibbs probability distribution of region maps is proportional to:

$$
P_{\mathbf{V}}(\mathbf{m}) \propto \exp \left(\sum_{\mathbf{r} \in \mathbf{R}} \sum_{a \in \mathbf{A}} \sum_{\boldsymbol{\nu} \in \mathbf{N}_{a}} V_{a}\left(m_{\mathbf{r}}, m_{\mathbf{r}+\boldsymbol{\nu}}\right)\right)
$$

This MGRF is identified by using the approximate analytical maximum likelihood estimates of the potentials [370,371]:

$$
\begin{aligned}
& V_{a, \text { eq }}=\frac{K^{2}}{K-1}\left(f_{\text {eq: } a}(\mathbf{m})-\frac{1}{K}\right)=4 f_{\text {eq: } a}(\mathbf{m})-2 \\
& V_{a, \text { ne }}=\frac{K^{2}}{K-1}\left(f_{\text {ne: } a}(\mathbf{m})-1+\frac{1}{K}\right)=4 f_{\text {ne: } a}(\mathbf{m})-2
\end{aligned}
$$

where $f_{\text {eq:a }}(\mathbf{m})$ and $f_{\text {ne: } a}(\mathbf{m})$ are empirical probabilities of the equal and non-equal labels pairs, respectively, in all the equivalent voxel pairs $\left\{(\mathbf{r}, \mathbf{r}+\boldsymbol{\nu}): \boldsymbol{\nu} \in \mathbf{N}_{a} ; \mathbf{r} \in \mathbf{R}\right\}$. The complete algorithm for modeling pairwise spatial interaction is illustrated in Algorithm 5 and an illustrative output is shown in Figure 18. 


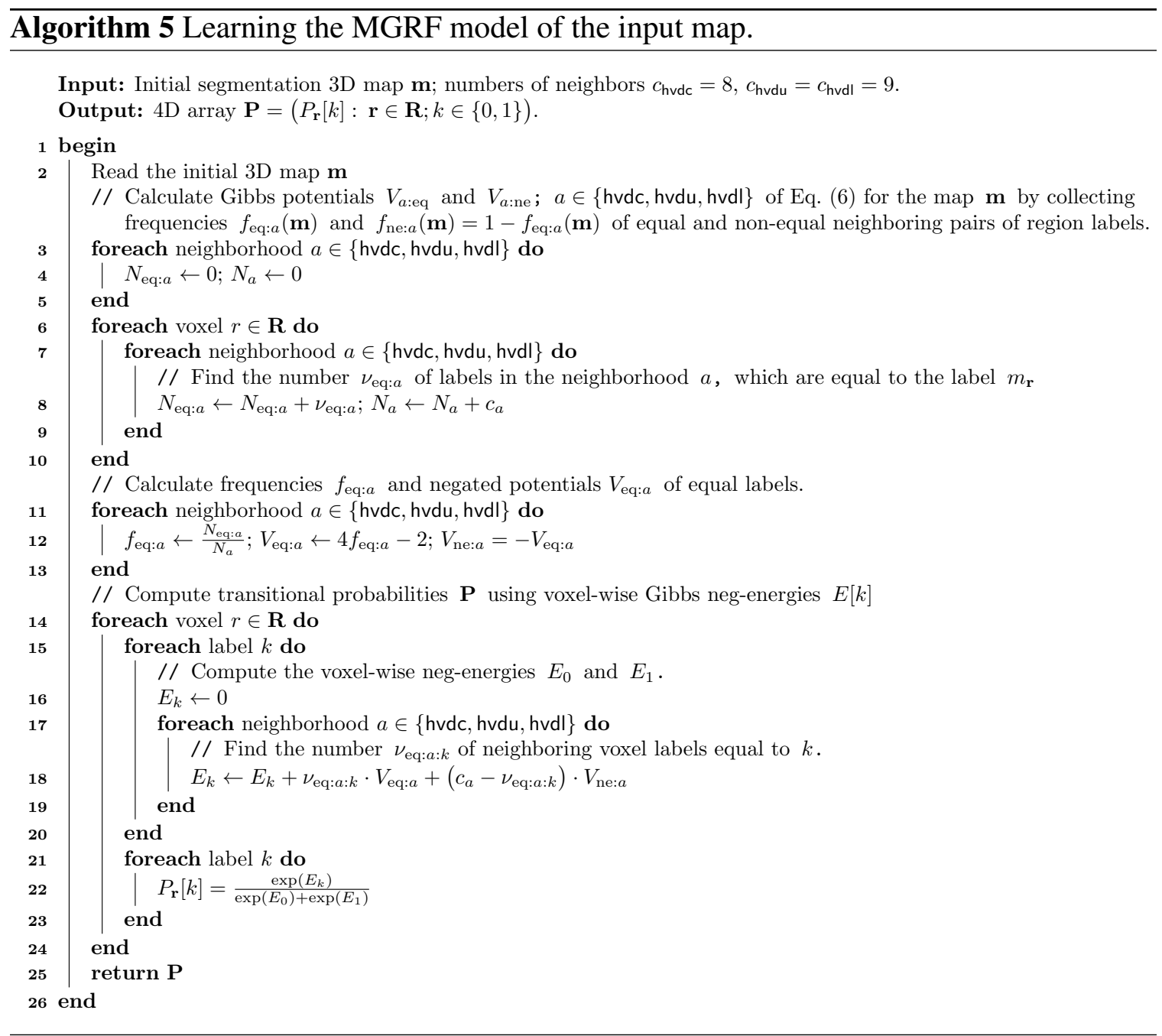

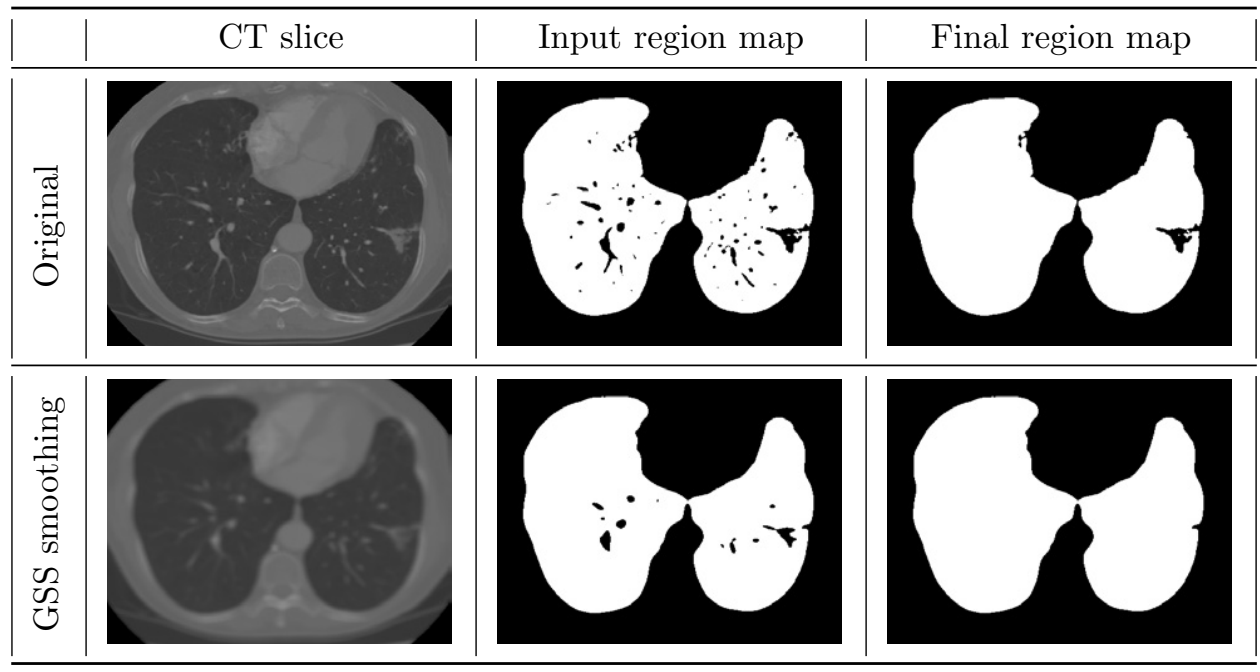

FIGURE 18: MGRF-guided segmentation of original image and its GSS smoothed version. 


\section{Joint MGRF model of 3D chest CT images}

To initially segment the lungs, the above unconditional adaptive shape prior, $P_{\mathrm{sh}}(\mathbf{m})$, conditional first-order appearance model, $P(\mathbf{g} \mid \mathbf{m})$, and unconditional second-order model, $P_{\mathbf{V}}(\mathbf{m})$ of spatial dependencies between the lung labels in Equations (8) and (9) are integrated into a joint MGRF model of either original, or GSS-smoothed images, g, and their region maps, m:

$$
P(\mathbf{g}, \mathbf{m})=P(\mathbf{g} \mid \mathbf{m}) \underbrace{P_{\mathrm{sh}}(\mathbf{m}) P_{\mathbf{V}}(\mathbf{m})}_{P(\mathbf{m})}
$$

Both the images and region maps are co-aligned to the subject-specific atlas defining the shape prior. The Bayesian maximum a posteriori estimate, $\mathbf{m}^{*}=\arg \max _{\mathbf{m}} L(\mathbf{g}, \mathbf{m})$, of the region map, given an image g, maximizes the log-likelihood

$$
L(\mathbf{g}, \mathbf{m})=\log P(\mathbf{g} \mid \mathbf{m})+\log P_{\mathrm{sh}}(\mathbf{m})+\log P_{\mathbf{V}}(\mathbf{m})
$$

\section{Classification using majority voting}

The majority voting, which is popular in fusing concurrent decisions, is a special case of the weighted voting. The final segmentation map, $\widehat{\mathbf{m}}$, combines by the majority voting the individual Bayesian region maps $\mathbf{m}$ and $\mathbf{m}_{l}$, having been built separately for the original image, $\mathbf{t}$, and its $L$ GSS-transformed versions, $\mathbf{t}_{l: \sigma_{l}} ; l=1, \ldots, L$ :

$$
\widehat{\mathbf{m}}_{\mathbf{r}}=\arg \max _{k \in\{0,1\}}\left\{\delta\left(k-m_{\mathbf{r}}\right)+\sum_{l=1}^{L} \delta\left(k-m_{l: \mathbf{r}}\right)\right\}
$$

The two GSS kernels: $l=1,9 \times 9 \times 5 ; \sigma_{1}^{2}=4.5$, and $l=2,17 \times 17 \times 5, \sigma_{2}^{2}=8.5-$ were used in the experiments below.

\section{Performance Evaluation Metrics}

The performance of the proposed lung segmentation framework is evaluated using two types of metrics: area-based similarity metrics and a distance-based error. The areabased similarity indicates the overlap between the segmented area and the ground truth. 
This type of metrics are important for studying area measurements, e.g., total lung volumes. The distance-based error measures how close edges of the segmented region are to the ground truth. Namely, it describes how accurate the shape of a segmented object is with respect to its ground truth. In this chapter, the Dice similarity coefficient (DSC) and the percentage volume difference (PVD) are used to describe the area-based similarity, while the 95-percentile bidirectional Hausdorff distance (BHD) is used to characterize the distance-based error metric. Both evaluation metrics are detailed below.

\section{Dice Similarity Coefficient (DSC)}

Let $G$ and $S$ denote sets of ground-truth and segmented lung voxels, respectively. The similarity volumetrics evaluates an overlap between these sets and account for cardinalities (i.e., voxel numbers) $c_{i}=\left|V_{i}\right|$ of true positive (tp), false positive (fp), and false negative (fn) subsets $V_{i} ; i \in\{\mathrm{tp}, \mathrm{fp}, \mathrm{fn}\}$, The subsets contain true lung voxels labeled the lungs, non-lung (background) voxels labeled the lungs, and true lung voxels labeled the background, respectively, (see Figure 19):

$$
\begin{array}{ll}
V_{\mathrm{tp}}=\{\mathbf{v}: \mathbf{v} \in G \text { AND } \mathbf{v} \in S\} ; & c_{\mathrm{tp}}=\left|V_{\mathrm{tp}}\right| \\
V_{\mathrm{fp}}=\{\mathbf{v}: \mathbf{v} \notin G \text { AND } \mathbf{v} \in S\} ; & c_{\mathrm{fp}}=\left|V_{\mathrm{fp}}\right| \\
V_{\mathrm{fn}}=\{\mathbf{v}: \mathbf{v} \in G \text { AND } \mathbf{v} \notin S\} ; & c_{\mathrm{fn}}=\left|V_{\mathrm{fn}}\right|
\end{array}
$$

Obviously, $G=V_{\mathrm{tp}} \cup V_{\mathrm{fn}} ; S=V_{\mathrm{tp}} \cup V_{\mathrm{fp}} ; V_{\mathrm{tp}}=G \cap S$, and $V_{\mathrm{tp}} \cup V_{\mathrm{fp}} \cup V_{\mathrm{fn}}=G \cup S$ where $\cup$ and $\cap$ denote the set union and intersection, respectively. Therefore, it holds that $|G|=c_{\mathrm{tp}}+c_{\mathrm{fn}} ;|S|=c_{\mathrm{tp}}+c_{\mathrm{fp}}$, and $|G \cup S|=c_{\mathrm{tp}}+c_{\mathrm{fp}}+c_{\mathrm{fn}}$. The Dice similarity coefficient (DSC) [376] is defined as:

$$
D S C=\frac{2 c_{\mathrm{tp}}}{2 c_{\mathrm{tp}}+c_{\mathrm{fp}}+c_{\mathrm{fn}}} \equiv \frac{2|G \cap S|}{|G|+|S|}
$$

The value of the DSC ranges from 0 to 1 , where 0 means that there is no similarity and 1 means that there is perfect similarity. To obtain the ground truth, the lung borders were delineated by a radiologist. 


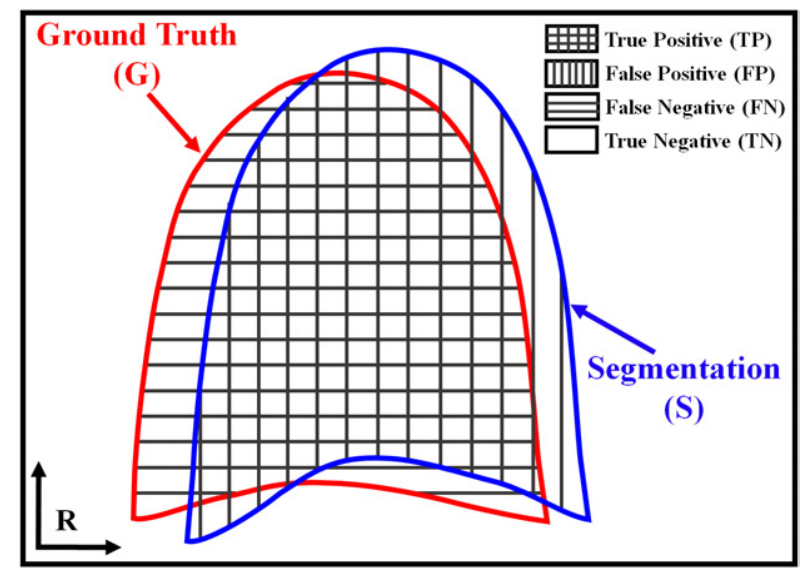

FIGURE 19: Coronal 2D schematic illustration of the determination of segmentation measures for calculating the Dice similarity coefficient (DSC).

2. overlap measure

The overlap between two binary segmentation volumes (e.g. left lung in groundtruth and segmented result) is defined as the volume of their intersection divided by the volume of their union.

$$
\text { overlap }=\frac{c_{t p}}{c_{t p}+c_{f p}+c_{f n}}
$$

The value of the overlap ranges from 0 to 1 , where 0 means that there is no similarity and 1 means that there is perfect similarity. It is very similar to DSC measure as it has an indication of the overlapping value. The overlap has been included as it used for the blind evaluation through the third party; namely, the LOLA11 orgganizers.

\section{3. percentage volume difference (PVD)}

Another area-based metric that is used in this chapter for the evaluation of segmentation, in addition to the DSC, is the percentage volume difference (PVD). The PVD is the percentage volume difference between the segmentation, $\mathrm{S}$, and the ground truth, $\mathrm{G}$, as 
seen in Figure 20:

$$
P V D=100 \frac{\left(c_{\mathrm{tp}}+c_{\mathrm{fn}}\right)-\left(c_{\mathrm{tp}}+c_{\mathrm{fp}}\right)}{c_{\mathrm{tp}}+c_{\mathrm{fn}}} \equiv 100 \frac{|G|-|S|}{|G|}
$$

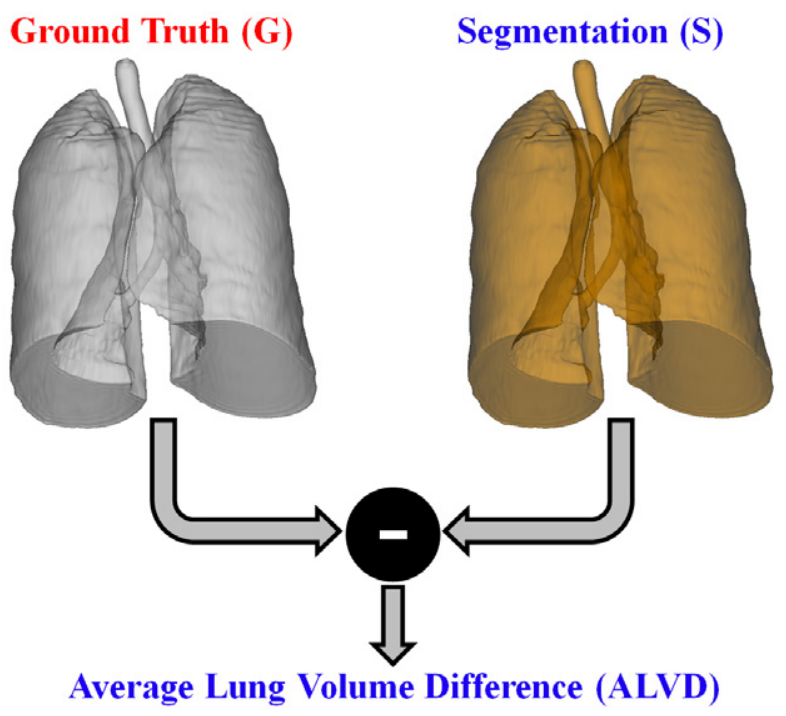

FIGURE 20: 3D schematic illustration for the absolute lung volume difference (ALVD) estimation.

\section{Bidirectional Hausdorff Distance (BHD)}

In addition to the DSC and PVD, the distances between $\mathbf{G}$ and $\mathbf{S}$ borders are used as an additional metric to measure the accuracy of the proposed segmentation approach. The 95-percentile bidirectional Hausdorff distance (BHD) is used to measure dissimilarities between the $G$ and $S$ boundaries. The HD from $G$ to $S$ is the maximum distance from the points $\mathfrak{g}$ from $G$ to their closest points $\mathfrak{s}$ in $S$ [377]:

$$
\operatorname{HD}_{G \rightarrow S}=\max _{\mathfrak{g} \in G}\left\{\min _{\mathfrak{s} \in S}\{d(\mathfrak{g}, \mathfrak{s})\}\right\}
$$

where $d(\mathfrak{g}, \mathfrak{s})$ is the Cartesian distance between two 3D points. The HD is asymmetric, as generally $\mathrm{HD}_{G \rightarrow S} \neq \mathrm{HD}_{S \rightarrow G}$. The symmetric BHD between these two sets is defined as

$$
\operatorname{BHD}_{G \leftrightarrow S}=\max \left\{\mathrm{HD}_{G \rightarrow S}, \mathrm{HD}_{S \rightarrow G}\right\}
$$


To decrease the sensitivity to outliers, the $95^{\text {th }}$-percentile BHD is used in this chapter to measure the segmentation accuracy.

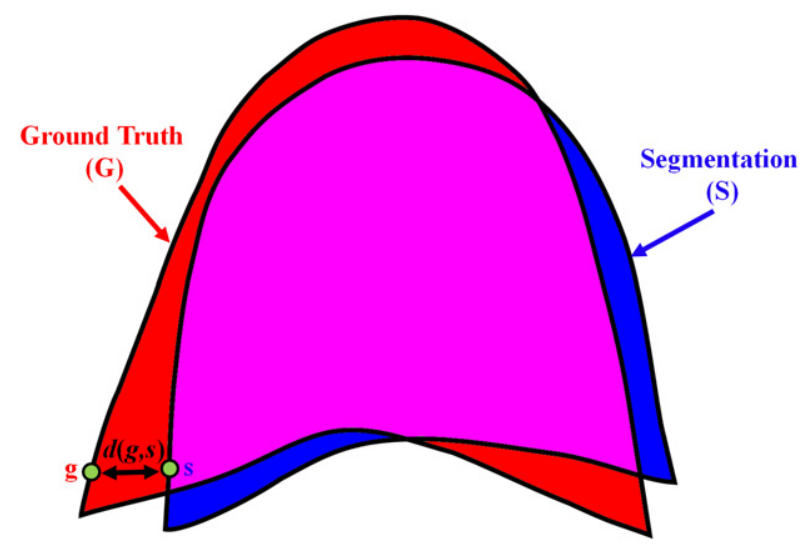

FIGURE 21: A schematic illustration for the Haussdorf distance (HD) calculation.

The BHD between the segmented region $\mathrm{S}$ and its ground truth $\mathrm{G}$ is defined as:

$$
\operatorname{BHD}(\mathbf{G}, \mathbf{S})=\max \{\mathrm{HD}(\mathbf{G}, \mathbf{S}), \operatorname{HD}(\mathbf{S}, \mathbf{G})\}
$$

\section{Experimental Results}

The segmentation accuracy and robustness of the presented framework were evaluated on the local collection of 3D CT chest images for 30 subjects, abbreviated UoLDB below, and two publicly available sets from the ISBI 2012 VESsel SEgmentation in the Lung (VESSEL) challenge [2,378] (20 subjects) and MICCAI 2011 LObe and Lung Analysis (LOLA11) challenge [3] (55 subjects). The images have been acquired with different scanners and data collection protocols and presented both normal lungs and various lung pathologies.

1. Evaluation using UoLDB.

The 50 (20 training and 30 test) UoLDB images of size from $512 \times 512 \times 270$ to $512 \times 512 \times 450$ have been collected with a multi-detector GE Light Speed Plus scanner 

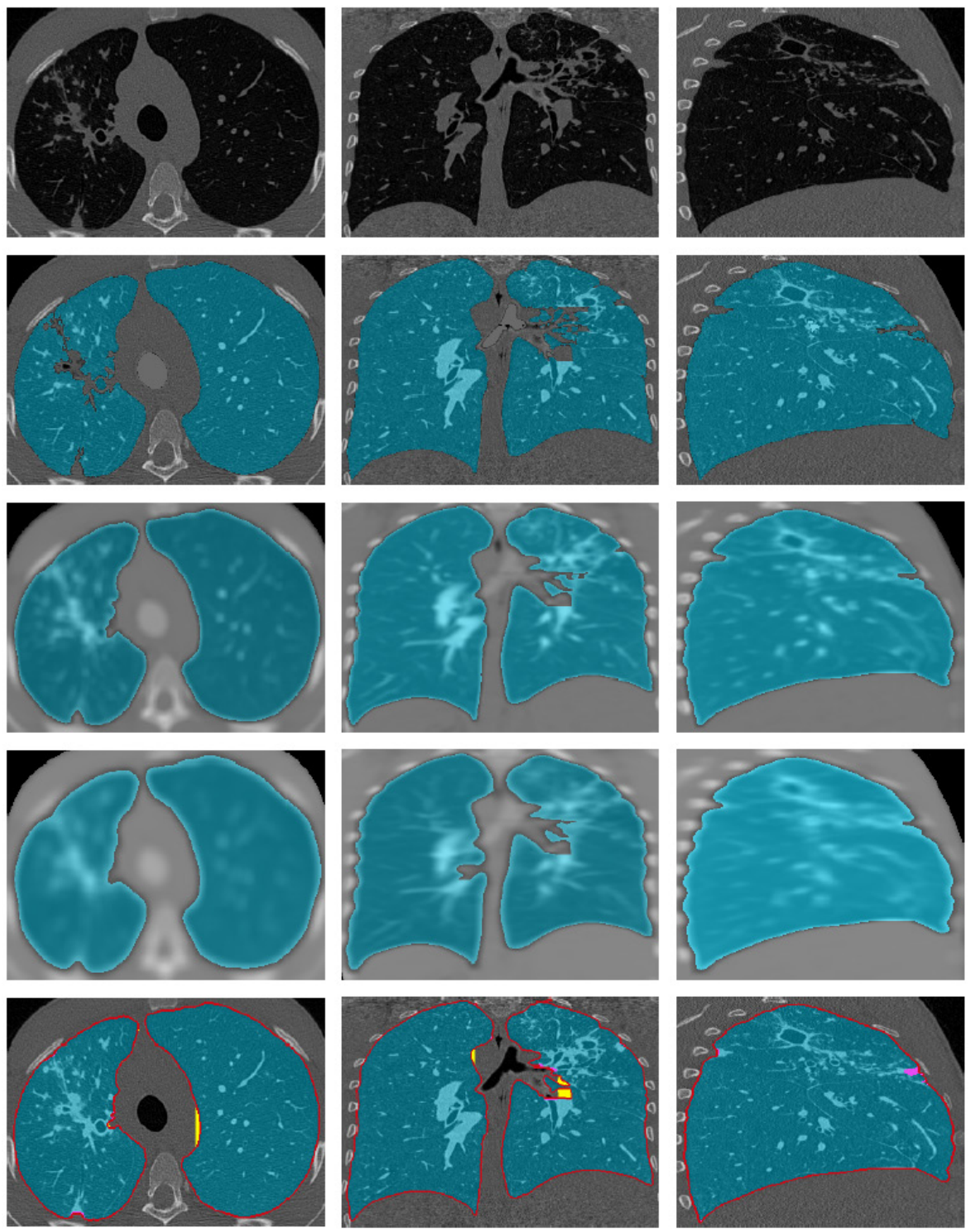

FIGURE 22: 3D lung segmentation (the UoLDB): 2D axial (first column), coronal (second column) and sagittal (third column) cross-sections of an original image (first row); its segmentation (second row); segmentation of the GSS-filtered images with $9 \times 9 \times 5$ (third row) and $17 \times 17 \times 5$ (forth row) kernels; and final segmentation (fifth row) with color-coded ground truth edges, false positive errors, and false negative errors (red, yellow, and pink points, respectively). Only these two kernels were used in the experiments, because after the second smoothing the overall segmentation accuracy improves insignificantly. 

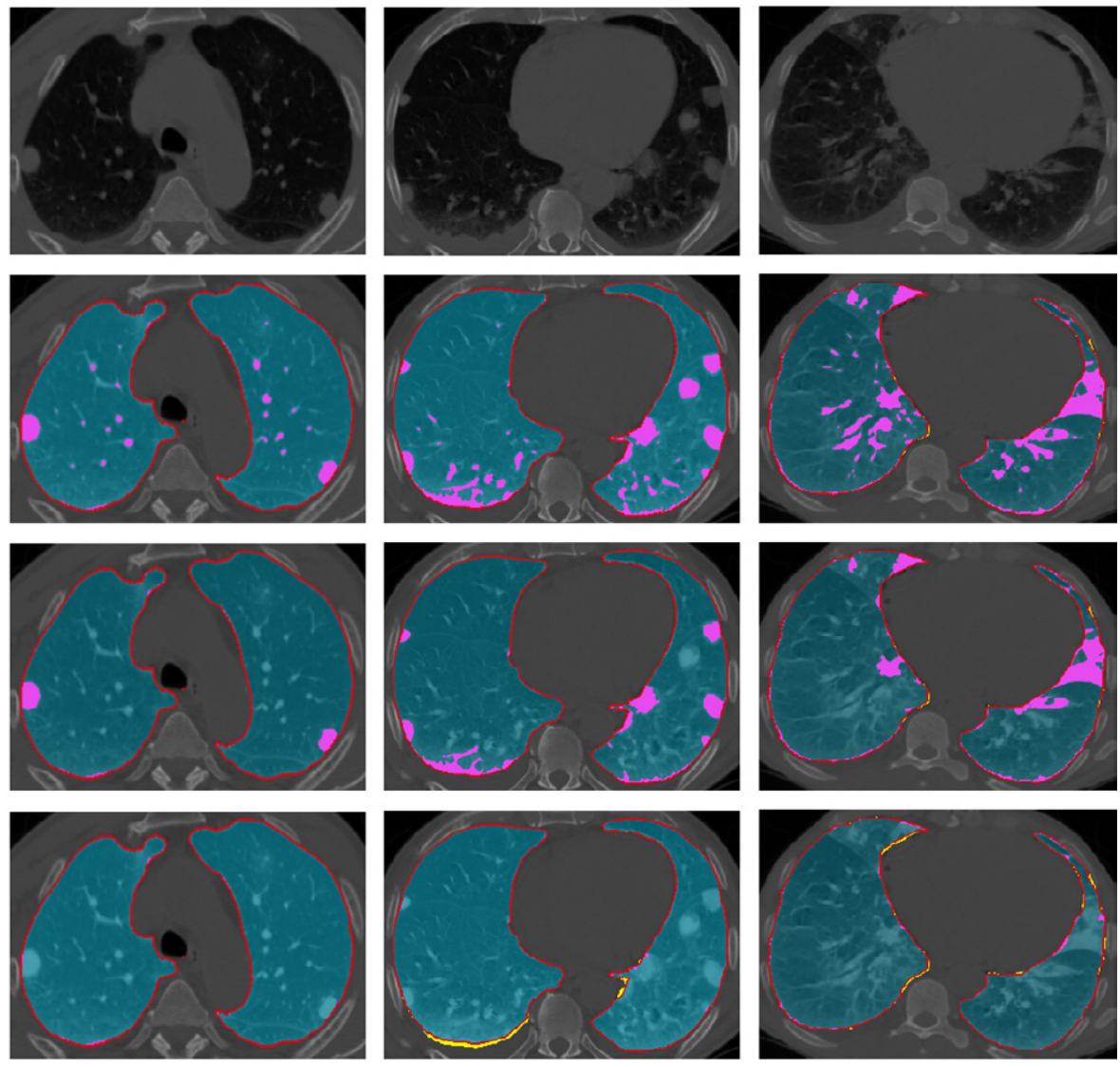

FIGURE 23: Impacts of elements of the proposed segmentation for 3 UoLDB subjects: original 2D chest sections (first row) and lung regions found for the original intensities and spatial interactions (second row); combined original/GSS-filtered intensities and spatial interactions (third row); and combined original/GSS-filtered intensities and spatial interactions, together with the adaptive shape model (fourth row). Same color ground truth and error coding as in Figure 22.

(General Electric, Milwaukee, USA) with the imaging protocols of $140 \mathrm{kV}$; $100 \mathrm{~mA}$; slice thickness from 0.703 to $2.50 \mathrm{~mm}$; scanning pitch 1.5, and field-of-view $36 \mathrm{~cm}$. Figure 22 details the 3D lung segmentation results for one subject from the UoLDB. The proposed segmentation employs only two GSS filtering stages because its overall accuracy improved insignificantly beyond the second stage in a few pilot experiments.

In order to highlight advantages of the integration of visual appearance descriptors 

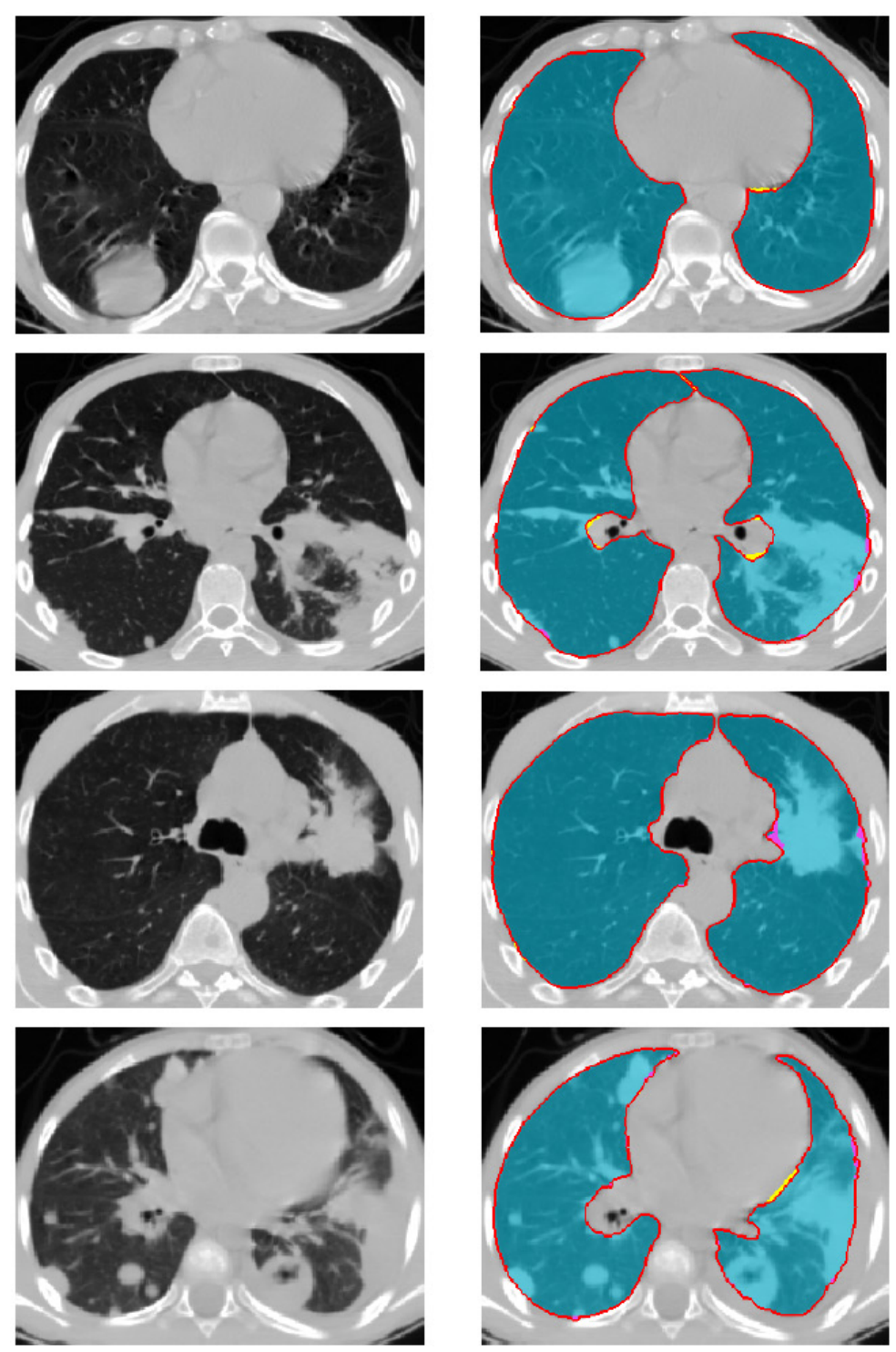

FIGURE 24: Other 4 subjects from the UoLDB: original CT chest images with different pathologies: (left: from top to bottom) lung mass; consolidation; lung cancer, and cavitation + consolidation pathologies, and the proposed segmentation (right: the same color ground truth and error coding as in Figure 22).

with the adaptive shape descriptor, the test UoLDB images were also segmented by combining only the visual appearance descriptors, characterizing the original and GSS-filtered intensities and their pairwise co-occurrences. The resulting accuracy for three UoLDB subjects in Figure 23 was low - due to similar original voxel intensities, the pathological lung 
tissues were included into the segmented chest, rather than lungs. The segmentation error decreased after adding the GSS-filtered appearance descriptors and improved even further after integrating all the appearance descriptors with the adaptive shape prior. More results for subjects with different types of pathologies are shown in Figure 24.
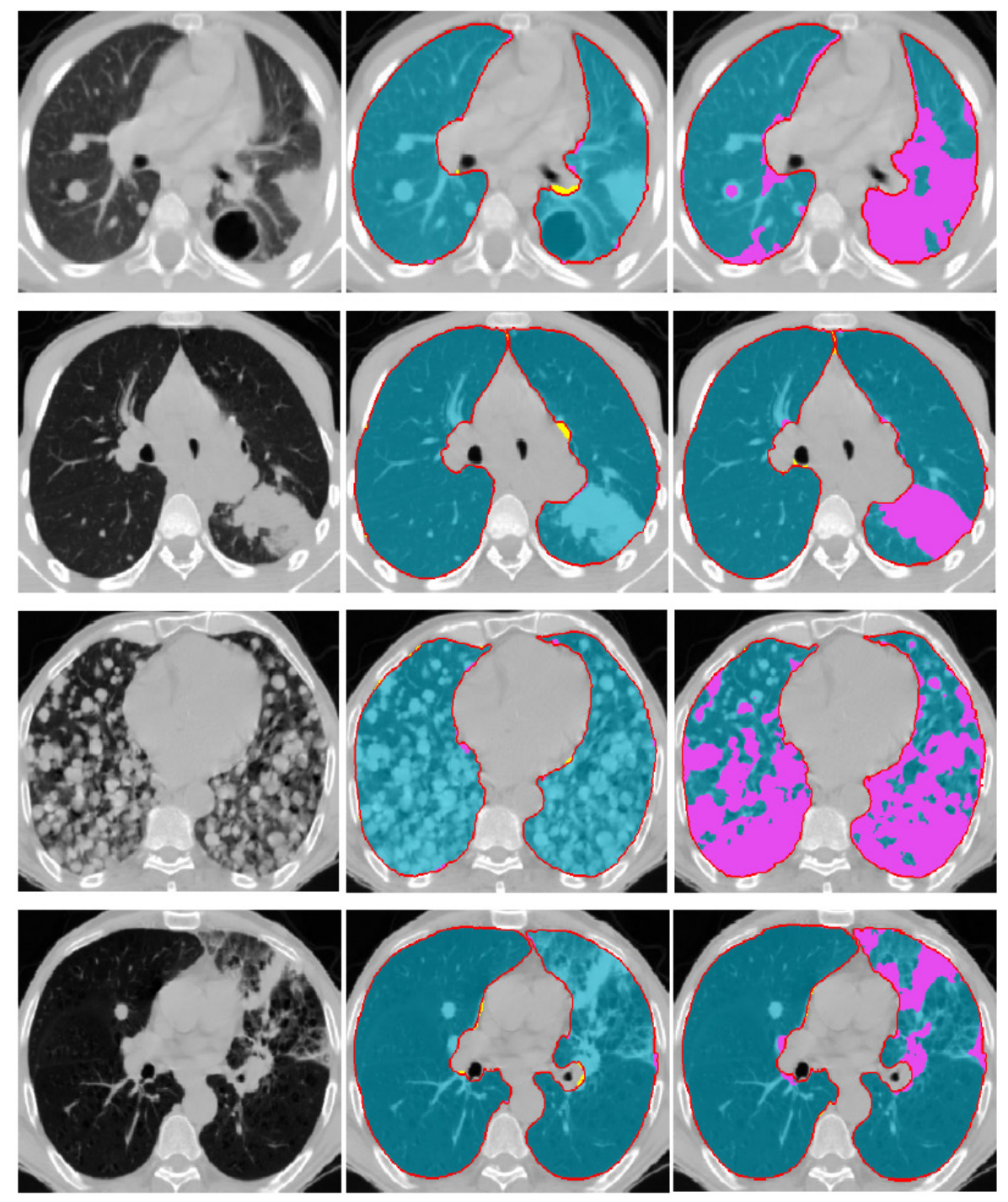

FIGURE 25: Comparative segmentation of the UoLDB: First column- original CT sections with, from top to bottom, plural effusion and lung nodules; consolidation; pulmonary sarcoidosis, and fibrosis; second column- the proposed segmentation, and third columnthe FC segmentation [1] (the same color ground truth and error coding as in Figure 22). 
TABLE 11: DSC, BHD, and PVD accuracy ( mean $\left._{ \pm \text {standar deviation }}\right)$ of different components (feature groups FG) of the proposed framework on the UoLDB in terms of the: FG1 (intensities and spatial interactions), FG2 (combined original / GSS-filtered intensities and spatial interactions), FG3 (FG2 + adaptive shape priors).

\begin{tabular}{|c|c|c|c|}
\cline { 2 - 4 } \multicolumn{1}{c|}{} & \multicolumn{3}{c|}{ UoLDB (30 test images) } \\
\cline { 2 - 4 } \multicolumn{1}{c|}{} & DSC, $\%$ & BHD, $\mathrm{mm}$ & PVD, \\
\hline FG1 & $0.932_{ \pm 0.032}$ & $13.2_{ \pm 4.6}$ & $9.90_{ \pm 7.20}$ \\
\hline FG2 & $0.966_{ \pm 0.017}$ & $9.53_{ \pm 2.92}$ & $2.90_{ \pm 0.70}$ \\
\hline FG3 & $0.984_{ \pm 0.010}$ & $2.20_{ \pm 1.00}$ & $0.42_{ \pm 0.10}$ \\
\hline$p$-value & $<0.0001$ & $<0.0001$ & $<0.0001$ \\
\hline
\end{tabular}

TABLE 12: DSC, BHD, and PVD accuracy (mean $\left.{ }_{ \pm \text {standar deviation }}\right)$ of the proposed method and FC segmentation [1] on the UoLDB and VESSEL [2] databases.

\begin{tabular}{|c|c|c|c|}
\cline { 2 - 4 } \multicolumn{1}{c|}{} & \multicolumn{3}{c|}{ UoLDB (30 test images) } \\
\cline { 2 - 4 } \multicolumn{1}{c|}{} & DSC, $\%$ & BHD, mm & PVD, \\
\hline Prop. segm. & $0.984_{ \pm 0.010}$ & $2.20_{ \pm 1.00}$ & $0.42_{ \pm 0.10}$ \\
\hline FC [1] & $0.906_{ \pm 0.098}$ & $19.0_{ \pm 9.9}$ & $15.4_{ \pm 15.3}$ \\
\hline$p$-value & $<0.0001$ & $<0.0001$ & $<0.0001$ \\
\hline & VESSEL $[2](20$ test images $)$ \\
\hline Prop. segm. & $0.990_{ \pm 0.005}$ & $2.10_{ \pm 1.60}$ & $0.39_{ \pm 0.20}$ \\
\hline FC [1] & $0.967_{ \pm 0.013}$ & $11.5_{ \pm 5.1}$ & $2.40_{ \pm 1.20}$ \\
\hline$p$-value & $<0.0001$ & $<0.0001$ & $<0.0001$ \\
\hline
\end{tabular}

The segmentation accuracy was evaluated for each test 3D CT image with the Dice similarity coefficient (DSC) [376], bidirectional Hausdorff distance (BHD), and percentage volume difference (PVD) [377], which characterize spatial overlaps, maximum surfaceto-surface distances, and volume differences, respectively, between the segmented and 
"ground-truth" lung regions. Table 11 summarizes the DSC, BHD, and PVD statistics for all the test UoLDB data to show the effect of different feature groups (FG) of the proposed framework. The ground-truth lung borders were outlined manually by a radiologist.

The accuracy of the proposed pathological lungs segmentation method is also highlighted by comparing its performance against the traditional fuzzy connectedness (FC)based lung segmentation [1], having a publicly available open-source software Lung Segmentation Tool (http://www.nitrc.org/projects/nihlungseg/). These experiments used the best performing manual seeding mode, recommended in its video tutorial default settings for refining segmented region maps: filling holes with a $3 \mathrm{~mm}$-diameter binary filter and checking fuzzy connectedness. Figure 25 compares the proposed and FC segmentation qualitatively on a representative set of four pathological lungs from the UoLDB. The DSC, BHD, and PVD statistics for the UoLDB in Table 12 show that the proposed segmentation, accounting for both short/long-range local and global lung properties, is more accurate. Differences between the metrics means for the proposed and FC segmentation are statistically significant by the paired $t$-test (corresponding $p$-values are below 0.0001).

\section{Evaluation using VESSEL12 database.}

To evaluate the proposed segmentation robustness to a chosen CT scanner and scanning protocol, the segmentation framework was tested against the FC framework on the VESSEL database [2] containing the CT chest images of mostly normal and pathological lungs. The VESSEL data sets were collected with different scanners and protocols. The CT scans were collected and anonymized from three different hospitals: the University Clinic of Navarra (Pamplona, Spain), University Medical Center Utrecht (Utrecht, The Netherlands), and Radboud University Nijmegen Medical Centre (Nijmegen, The Netherlands). The data included both clinical exams taken for a variety of diagnosis, and CT scans for two lung cancer screening trials: the Dutch-Belgian randomized controlled lung cancer CT 
screening trial, (NELSON), and the International Early Lung Cancer Action program, (IELCAP) [378]. The ground truth for this database was provided by the ISBI 2012 VESSEL challenge organizers. More details can be found in [378]. Figure 26 qualitatively compares the proposed frameworks with the (FC)-based lung segmentation [1], and the DSC, BHD, and PVD statistics for segmenting the whole VESSEL database are presented in Table 12. It is clear from both the quantitative and qualitative assessment that the proposed framework achieves a higher accuracy, even if the majority of the data is healthy subjects.
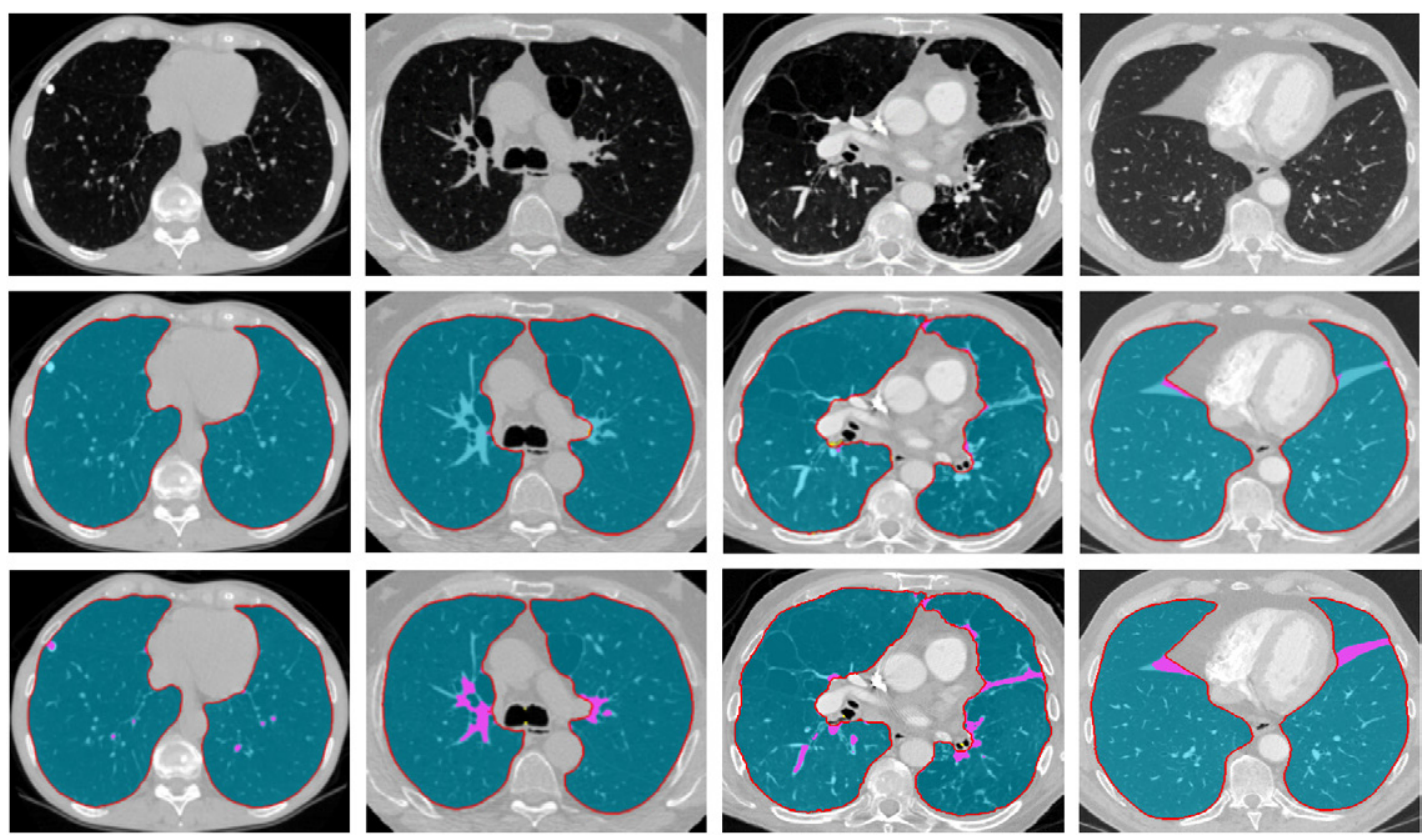

FIGURE 26: Comparative segmentation of the VESSEL [2] database: original CT sections (first row); The proposed segmentation (second row); and the FC segmentation (third row) [1] (the same color ground truth and error coding as in Figure 22).

3. Evaluation using LOLA11 database.

To provide a blind and fair comparison, a third party evaluation has been used through the participation in the LOLA11 challenge. The segmentation framework has been tested on the LOLA11 database [3], acquired by different scanners and protocols for 55 
subjects with diverse lung pathologies.

The LOLA11 organizers evaluate the lung segmentations for left and right lungs in terms of the overall overlap measure. The results has been reported in terms of mean, standard deviation, minimum, first quartile, median, third quartile, and maximum overlap over the 55 scans for each object separately. The overall score for the lung segmentation is the mean of the means. To follow the LOLA11 challenge regulations, the trachea and main bronchi have been removed from the LOLA11 image by customized region growing, which terminates after the trachea starts to split into four components. Then, if necessary, the lungs were separated with the optimal path finder [379] searching for intensity differences. The search is conducted on a $2 \mathrm{D}$ axial slice after finding a single connected component with a lung volume being nearly equal to the volume of the lung components in the previous slice. The finder connects two automatically initialized points in the chest region that separates the left and right lungs, as described in [379]. Figure 27 shows an example slice together with its calculated pixel-wise energies to search for the optimum path between the two automatically initialized points and the separation path found. The
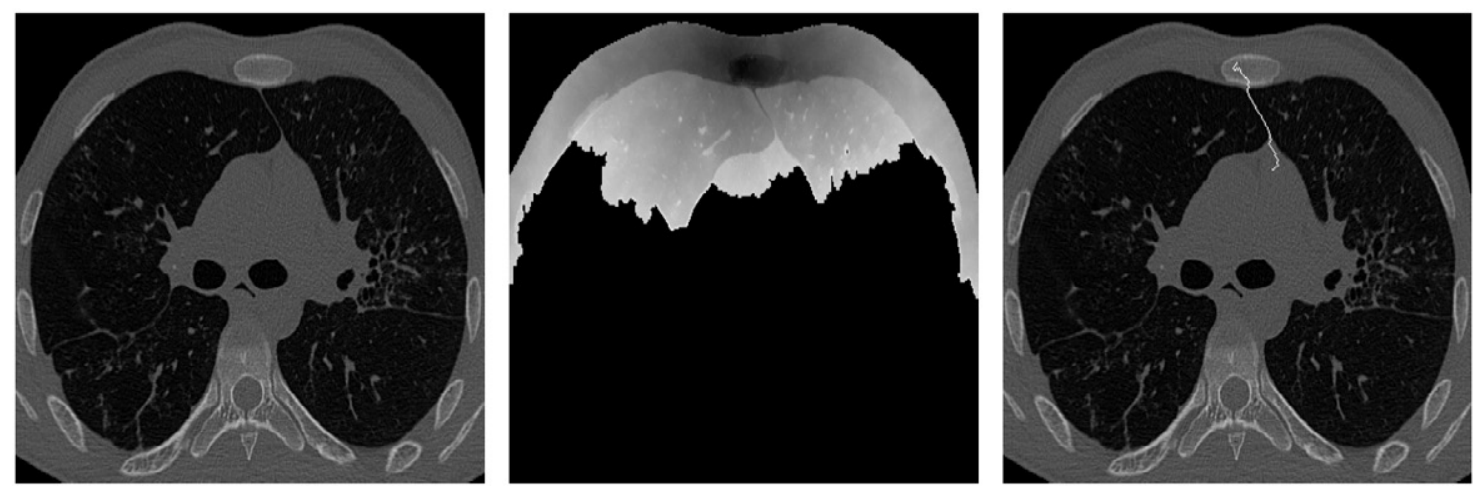

FIGURE 27: Lung separation process; (a) an original slice; (b) generated energies of pixels traversed until finding the optimum path, and (c) the original image with the overlaid separating path.

LOLA11 challenge organizers blindly evaluated the proposed segmentation framework, re- 
sults are detailed in Table 13, and compared the results, exemplified in Figure 28, to the ground truth and reported the mean right and left lungs overlaps over all the 55 scans. Table 14 and Figure 29 compare the top-rank LOLA11 methods, the proposed segmentation results having the first rank out of the 16 competitors.

TABLE 13: Right and left lung overlap scores for each subject in LOLA11 challenge

\begin{tabular}{|c|c|c|}
\hline Scan & Left Lung & Right Lung \\
\hline lola11-01 & 0.9963 & 0.9983 \\
\hline lola11-02 & 0.9909 & 0.8890 \\
\hline lola11-03 & 0.9890 & 0.9922 \\
\hline lola11-04 & 0.9910 & 0.9925 \\
\hline lola11-05 & 0.9918 & 0.9952 \\
\hline lola11-06 & 0.9603 & 0.9954 \\
\hline lola11-07 & 0.9927 & 0.9944 \\
\hline lola11-08 & 0.9970 & 0.9961 \\
\hline lola11-09 & 0.9913 & 0.9981 \\
\hline lola11-10 & 0.9937 & 0.9951 \\
\hline lola11-11 & 0.9967 & 0.9941 \\
\hline lola11-12 & 0.9970 & 0.9960 \\
\hline lola11-13 & 0.9960 & 0.9978 \\
\hline lola11-14 & 0.9934 & 0.9945 \\
\hline lola11-15 & 0.9904 & 0.9929 \\
\hline lola11-16 & 0.9956 & 0.9976 \\
\hline lola11-17 & 0.9982 & 0.9966 \\
\hline lola11-18 & 0.9979 & 0.9973 \\
\hline
\end{tabular}


TABLE 13 - Overlap scores - Continue

\begin{tabular}{|l|l|l|}
\hline Scan & Left Lung & Right Lung \\
\hline lola11-19 & 0.9857 & 0.9968 \\
lola11-20 & 0.9029 & 0.9902 \\
lola11-21 & 0.9790 & 0.9876 \\
lola11-22 & 0.9985 & 0.9973 \\
lola11-23 & 0.9913 & 0.9928 \\
lola11-24 & 0.9909 & 0.9900 \\
lola11-25 & 0.9953 & 0.9965 \\
lola11-26 & 0.9992 & 0.9948 \\
lola11-27 & 0.9952 & 0.9894 \\
lola11-28 & 0.9945 & 0.9952 \\
lola11-29 & 0.9982 & 0.9949 \\
lola11-30 & 0.9933 & 0.9861 \\
lola11-31 & 0.9231 & 0.9983 \\
lola11-32 & 0.9893 & 0.9957 \\
lola11-33 & 0.9915 & 0.9952 \\
lola11-34 & 0.9960 & 0.9955 \\
lola11-35 & 0.9874 & 0.9789 \\
lola11-36 & 0.9852 & 0.9951 \\
lola11-37 & 0.9859 & 0.9882 \\
lola11-38 & 0.9934 & 0.9960 \\
lola11-39 & 0.9892 & 0.9957 \\
lola11-40 & 0.9945 & 0.9976 \\
lola11-41 & 0.9874 & 0.9926 \\
lola11-42 & 0.9853 & 0.9961 \\
\hline
\end{tabular}


TABLE 13 - Overlap scores - Continue

\begin{tabular}{|l|l|l|}
\hline Scan & Left Lung & Right Lung \\
\hline lola11-43 & 0.9939 & 0.9969 \\
lola11-44 & 0.9889 & 0.0515 \\
lola11-45 & 0.8735 & 0.9911 \\
lola11-46 & 0.9844 & 0.9876 \\
lola11-47 & 0.9931 & 0.9969 \\
lola11-48 & 0.9782 & 0.9870 \\
lola11-49 & 0.9984 & 0.9957 \\
lola11-50 & 0.9908 & 0.9761 \\
lola11-51 & 0.9903 & 0.9946 \\
lola11-52 & 0.9852 & 0.9765 \\
lola11-53 & 0.9956 & 0.9940 \\
lola11-54 & 0.9862 & 0.9952 \\
lola11-55 & 0.9915 & 0.9921 \\
\hline
\end{tabular}



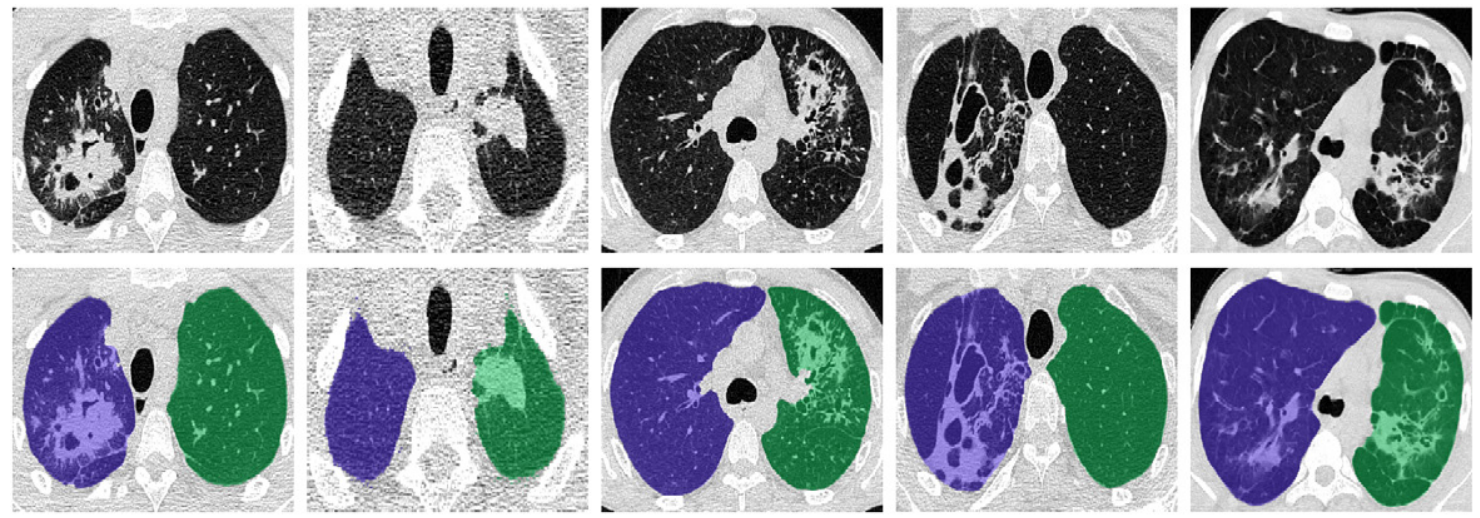

FIGURE 28: Segmenting pathological lungs from the LOLA11 database. First rowOriginal CT sections with, from left to right, fibrosis, lung mass (tumor), consolidation, cavitation + fibrosis, and fibrosis, and the proposed segmentation is in the second row (its accuracy has been evaluated by the LOLA11 organizers [3]).

TABLE 14: Mean overlap of the proposed segmentation method with the ground truth for the entire LOLA11 database of 55 chest CT images vs. the $2^{\text {nd }}-5^{\text {th }}$-rank LOLA11 competitors (these accuracies were provided by the LOLA11 organizers [3]).

\begin{tabular}{|c||c|c|c|c|c|c|c|c||c|}
\hline Method & obj & mean & std & min & Q1 & median & Q3 & max & score \\
\hline \multirow{2}{*}{ Prop. segm. } & LL & 0.986 & 0.022 & 0.874 & 0.987 & 0.992 & 0.995 & 0.999 & $\mathbf{0 . 9 8 0}$ \\
& RL & 0.974 & 0.128 & 0.052 & 0.991 & 0.995 & 0.996 & 0.998 & \\
\hline \multirow{2}{*}[380]{} & LL & 0.974 & 0.097 & 0.277 & 0.987 & 0.992 & 0.995 & 0.999 & .973 \\
& RL & 0.972 & 0.135 & 0.000 & 0.991 & 0.994 & 0.996 & 0.999 & \\
\hline \multirow{2}{*}[381]{} & LL & 0.971 & 0.093 & 0.309 & 0.982 & 0.988 & 0.992 & 0.997 & $\mathbf{0 . 9 7 0}$ \\
& RL & 0.969 & 0.134 & 0.000 & 0.986 & 0.990 & 0.993 & 0.998 & \\
\hline \multirow{2}{*}[382]{} & LL & 0.968 & 0.134 & 0.000 & 0.985 & 0.990 & 0.993 & 0.998 & $\mathbf{0 . 9 6 9}$ \\
& RL & 0.970 & 0.134 & 0.000 & 0.988 & 0.992 & 0.994 & 0.998 & \\
\hline & LL & 0.968 & 0.097 & 0.316 & 0.979 & 0.987 & 0.995 & 0.999 & $\mathbf{0 . 9 6 8}$ \\
& RL & 0.968 & 0.134 & 0.000 & 0.984 & 0.990 & 0.997 & 0.999 & \\
\hline
\end{tabular}




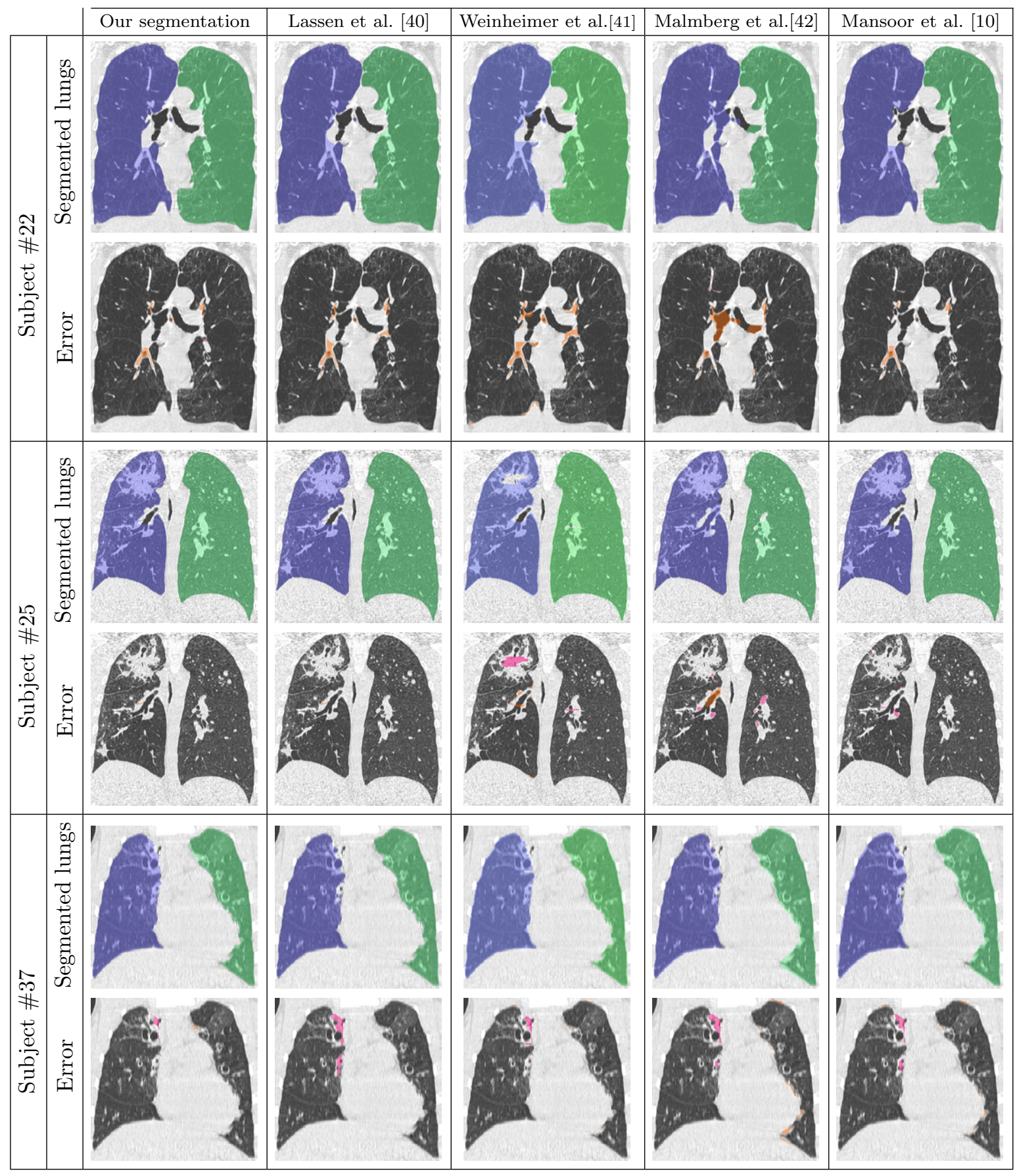

FIGURE 29: Proposed segmentation result for 3 subjects from the LOLA11 database vs. the $2^{\text {nd }}$ - to $5^{\text {th }}$-rank LOLA11 competitors (the LOLA11 organizers [3] compared the extracted lungs with their ground truth and accounted for differences, being orange/pinkcoded in the above region maps, to evaluate the overall accuracy). 


\section{E. Summary and Discussion}

Accurate segmentation of lung images, especially in the case of pathological lungs with, e.g., lung nodules, is essential for reliable CAD of pulmonary and lung diseases. Most segmentation errors are due to missing pathological tissues; however, these errors negligibly affect the overall segmentation accuracy because the pathological tissue volume is often very small compared to the whole lung volume, as in Figure 30 (left column). Nonetheless, segmentation inaccuracies of these types of tumors are a serious problem, because the undetected and non-localized tumor candidates will compromise the further analysis, e.g., detection and diagnostics, in any CAD system and preclude the early cancer diagnosis of a patient. Even though missing the tumor in Figure 30 (left column) reduces the overall DSC by only $0.1 \%$, it may explain the small difference $(0.7 \%)$ between the mean overlap scores for the proposed segmentation and the closest competitor on the LOLA11 challenge database of pathological lungs. Moreover, if a severe lung pathology affects a larger portion of the total lung volume, the inaccurate segmentation of these tissues will considerably reduce the overall accuracy, such as in Figure 30 (right column) where the overall DSC has decreased by $30.4 \%$. Therefore, accurate lung segmentation for various pathologies is a must for choosing a proper region of interest for subsequent CAD steps.

The methodology proposed in this chapter can accurately segment a wide range of pathological lungs from the 3D chest CT scans due to the combining first- and secondorder probabilistic descriptors of the original and GSS-smoothed images with the proposed appearance-guided adaptive shape prior. The latter requires a reasonably large training set of healthy and pathological lungs. To be accurately aligned, both the right and left lungs in each input CT image should have some healthy tissues to be used as guiding landmarks for alignment. Therefore, too small healthy sections in either the left or right lung will affect the proposed segmentation performance, as demonstrated in Figure 31. To extend the scope of the proposed segmentation technique in the future, other chest landmarks will be appended to be used with the healthy tissue. 

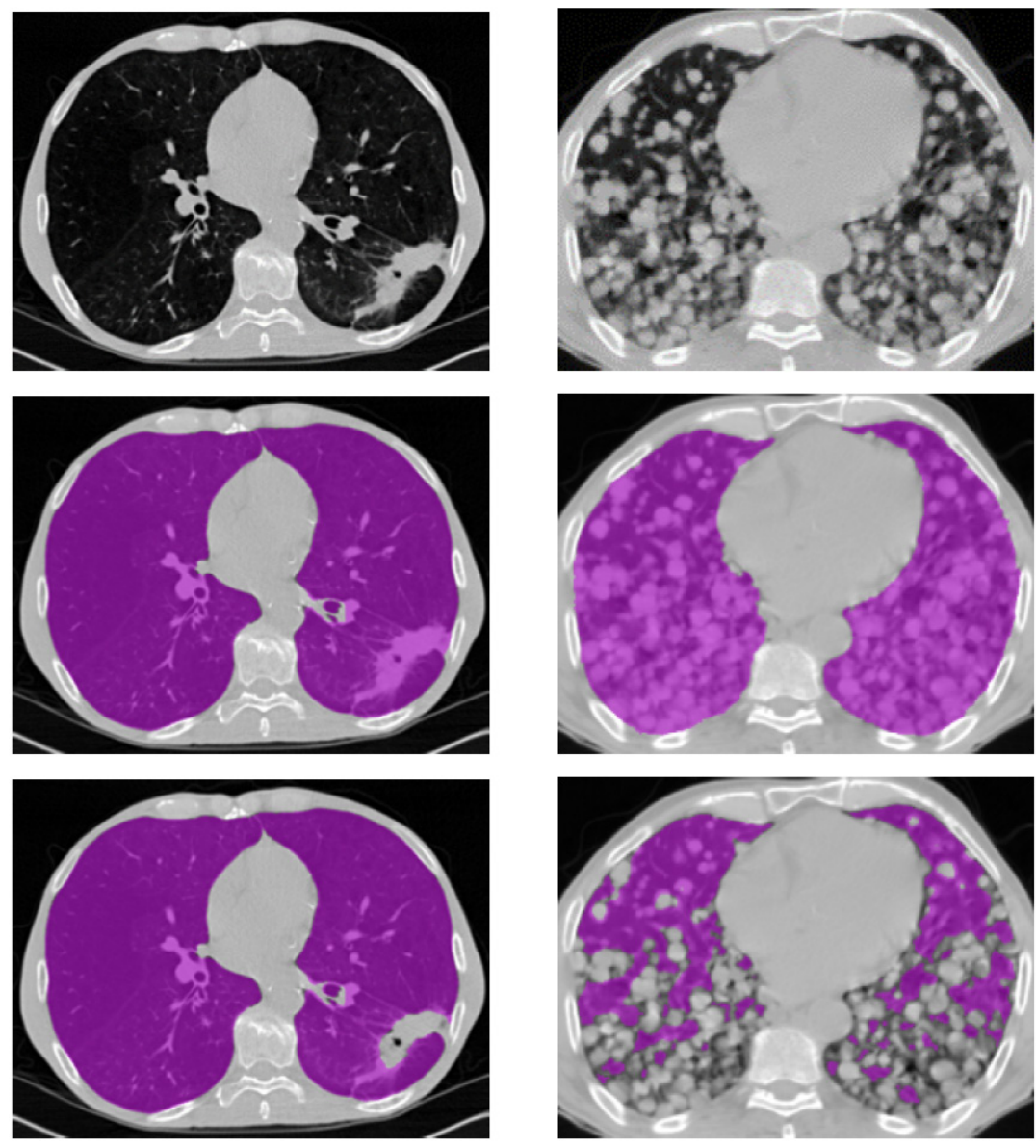

FIGURE 30: Total accuracy reduction due to erroneous segmentation of pathological tissues (first row) between ground truth (second row) and pathological-missing segmentation (third row): only $0.1 \%$ for missing the lung mass for a tumor (left column), but about $30 \%$ for a severe pathology (right column).

The high DSC, MHD, and PVD accuracy of the proposed lung segmentation framework has been confirmed experimentally on multiple in-vivo 3D CT image datasets. What is most importantly, the blind comparison with the publicly-available LOLA11 database has validated the superior performance of the proposed framework over many existing methods in segmenting various pathological lung images, ranging from mild to severe cases, such as pulmonary fibrosis, pleural effusion, consolidation, and cavities. These wider capabilities stem from using the adaptive prior shape model of the lungs to derive more accurate visual 

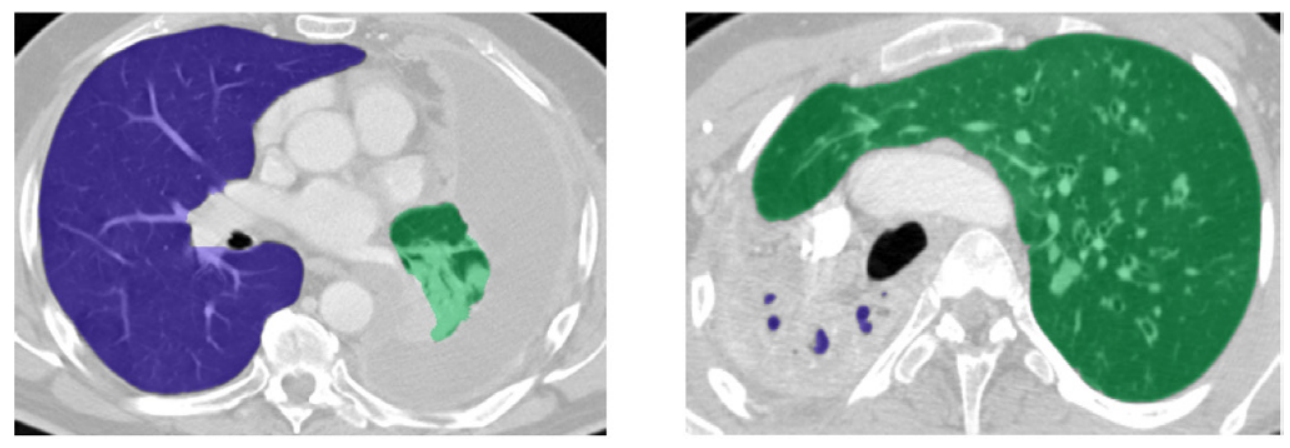

FIGURE 31: Impacts of severe right and left lung pathologies on the proposed segmentation accuracy: the ground-truth-to-left-lung overlaps of $87.4 \%$ (left) and 5.2\% (right), the true lung maps being available to only the LOLA11 organizers.

appearance descriptions of the chest CT images. The resulting higher accuracy can be beneficial for any current CAD system that uses lung segmentation as a critical initial step for measuring or estimating various biomarkers, such as a whole lung volume, left and right lungs volumes, and so forth [383]. These biomarkers assist in diagnosing many restrictive / obstructive lung diseases, such as interstitial lung disease, chronic obstructive pulmonary disease, extrinsic disorders, and asthma. Finally, the proposed framework can enhance the accuracy of any lung CAD system, e.g., the one for early detection of lung nodules because the correctly extracted lung fields constrain and accurately identify nodules' search space. The work presented in this chapter has been published in the IEEE Transactions on Medical Imaging [384], the International Symposium on Computational Models for Life Sciences (CMLS) [385], the International Symposium on Biomedical Imaging (ISBI) [386], and the International Conference on Image Processing (ICIP) [387, 388]. 


\section{CHAPTER IV \\ AN ISO-SURFACES BASED LOCAL DEFORMATION HANDLING FRAMEWORK OF LUNG TISSUES}

Handling the deformations of the lung tissues in successive chest computed tomography $(\mathrm{CT})$ scans of a patient is a vital step in any computer-aided diagnostic (CAD) system for lung cancer diagnosis. In this chapter, a new nonrigid registration methodology for the segmented lung region from CT data is proposed and involves two steps. The first step globally aligns the target-to-reference CT scans using an affine transformation based on ascent maximization of the estimated mutual information of the calculated distance map using the fast marching level sets method inside the segmented lung for both the target and reference objects. The second step is the local alignment of the target lung object in order to correct for intricate relative deformations due to breathing and heart beats. The local deformations are handled based on displacing each voxel of the target over evolving closed equi-spaced surfaces (iso-surfaces) to closely match the reference object. In order to displace the voxel on the iso-surfaces of the target lung object, the initial voxel-to-voxel match between target and reference lung objects is estimated by solving the 3D Laplace equation between each two corresponding iso-surfaces on the reference and target objects. Finally, the estimation of voxel-to-voxel match is refined through iterative energy minimization using a generalized Gauss-Markov random field (GGMRF) model. Qualitative and quantitative results on both in-vivo and simulated data demonstrate the promise of the proposed nonrigid registration framework.

\section{A. Introduction}

Lung registration is one of the most vital steps in developing any computer-aided 
diagnostic (CAD) system for the diagnosis of lung cancer, e.g., a CAD system for accurate estimation of lung nodules' growth rate. Lung CT registration is a challenging task due to large deformations resulted from breathing and heart beats, sliding motion between organs, and changes of image intensities as a result of compression. In the literature, different registration techniques have been proposed to handle lung deformations between successive CT scans. Most of the existing approaches exploit corresponding local structural elements (features) in the images. In particular, Castillo et al. [389] developed an optical flow deformable image registration method based on recovering parameterized voxel trajectories of the 4D thoracic CT scans. In order to reduce noise effects and image artifacts, the image intensity values are approximated on a variable grid by $3 \mathrm{D}$ cubic-splines. Then, the parameterized trajectory of each voxel is calculated by utilizing a cubic path assumption using a nonlinear least-squares fit to a local compressible flow equation. [390]. A simultaneous registration and segmentation of the lung from serial CT data was proposed by Zheng et al. [391]. Their joint framework is based on a 3D cubic B-Spines based nonrigid transformation for lung deformation and rigid structure for the lung tumors to preserve the volume and the shape of the tumor during the registration. Their method employed the sum of squared differences (SSD) as the similarity measure. Metz et al. [392] handled the motion estimation in dynamic medical imaging through the use of Lagrangian $\mathrm{nD}+\mathrm{t}$ transformation model parameterized by B-splines. Their registration framework employed a cost function to eliminate a bias towards a chosen reference frame, and used a global optimization routine as an optimization function. A smoothness constraint that depends on the expected motion of the organ and motion distortion due to pathology is applied to account for image noise and artifacts. Mattias et al. [393] proposed a Markov random field (MRF)-based deformable registration approach for lung CT using a symmetric diffeomorphic B-spline transformation model with diffusion regularization and introduced a stochastic sampling approach as a cost function between images to overcome the problems of continuous ones. A non-linear image gradient-based registration through the analysis 
of mathematical structure of the intensity independent normalized gradient field (NGF) distance was proposed by Konig et al. [394]. The NGF measures the angle between reference and template image intensity gradients at each point with the aim of image gradients alignment. The lower the values, the more parallel the gradients, and maximum values are obtained by orthogonal gradients.

In total, the above brief overview shows the following limitations of the existing techniques: $(i)$ most of them depend on intensity (grey scale) for registration, which can vary widely between successive scans due to different scanner types and image acquisition protocols; (ii) all existing techniques do not take into account the lung geometry or the anatomy; (iii) some of these approaches do not handle the global alignment; and (iv) none of the them guarantee a voxel-on-voxel match of the co-aligned lung data, which is an essential step in some clinical applications, such as the study of lung tissue perfusion using dynamic contrast-enhanced CT. To overcome these limitations, a topology preserving deformation handling framework has been proposed that exploits geometrical lung features by deforming it over a set of evolving iso-surfaces, rather than using only image intensities. Details of the proposed framework are outlined below.

\section{B. Methods}

The proposed framework for successive CT data registration is schematized in Fig-

ure 32. In this chapter, there is a focus on the accurate co-alignment of segmented lung objects for intra- and inter-patients data. The segmented lungs are obtained using the developed lung segmentation framework in Chapter III

\section{Global Alignment}

In order to reduce the variability of the segmented lung objects and maximize their overlap, successive CT scans are globally co-registered using a 3D affine transformation 


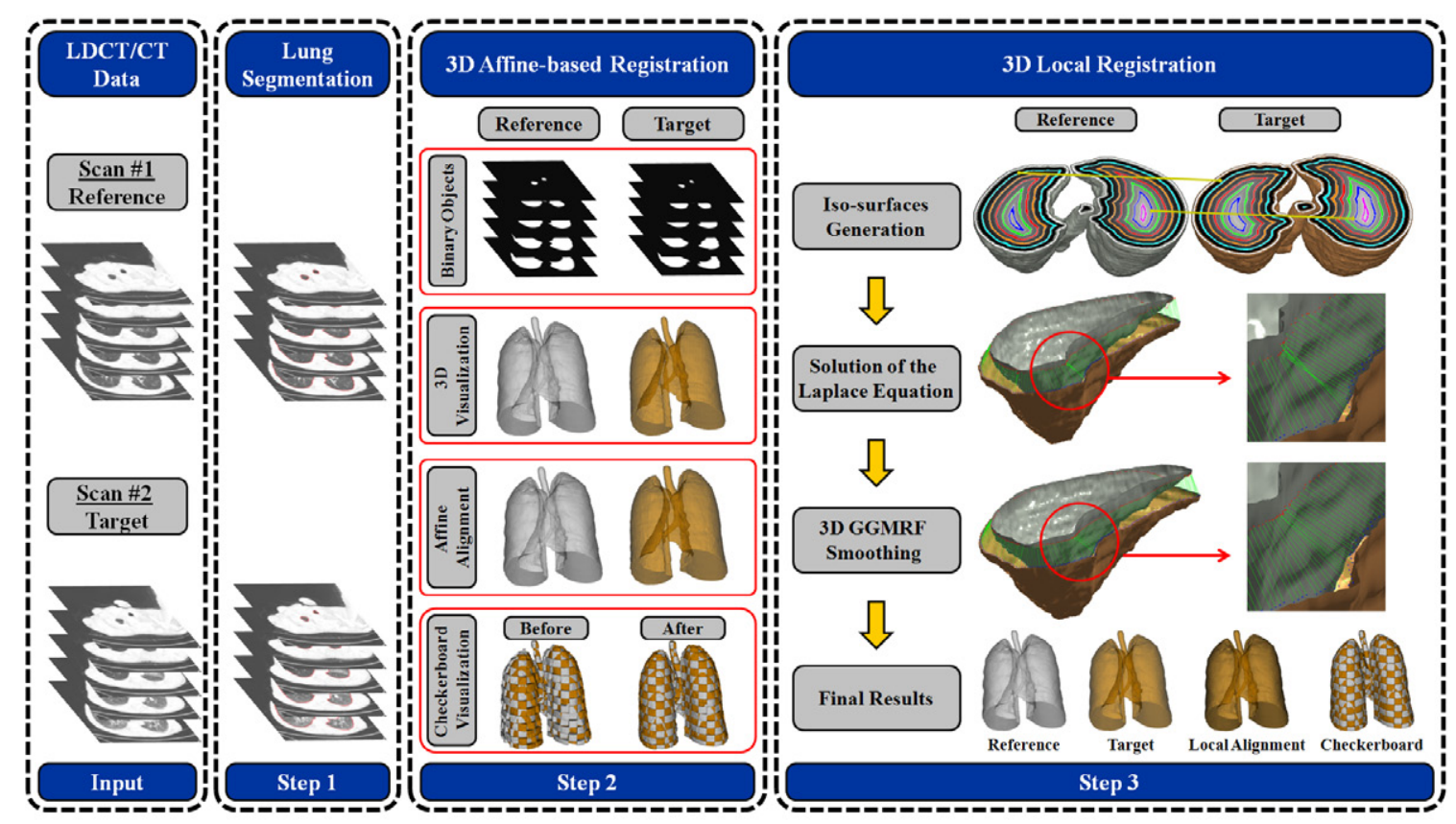

FIGURE 32: The proposed framework for the co-alignment of lung CT images.

model [395]. In order to perform global registration, the distance maps inside the reference and target lung objects are generated by finding the minimum Euclidean distance for every inner voxel to the object boundary using the fast marching level set [396].

Then, a 3D affine transformation with 12 degrees of freedom (3 for 3D translation, 3 for 3D rotation, 3 for 3D scaling, and 3 for 3D shearing); Equation (20) is applied to align the target object to the reference by maximizing the mutual information (MI) of the generated distance maps [395]. consider a point $\mathrm{x}=(x, y, z)$.

$$
\mathbf{x}^{\prime}=(R H S T) \mathbf{x}=M \mathbf{x}
$$

Where $T$ is the translation in the $x, y$, and $z$ directions that is given by:-

$$
T=\left[\begin{array}{cccc}
1 & 0 & 0 & t_{x} \\
0 & 1 & 0 & t_{y} \\
0 & 0 & 1 & t_{z} \\
0 & 0 & 0 & 1
\end{array}\right]
$$




$$
\begin{aligned}
& \text { and } R \text { is the rotation about } x, y \text {, and } z \text { axis given by:- } \\
& R_{x}=\left[\begin{array}{cccc}
1 & 0 & 0 & 0 \\
0 & \cos \theta & -\sin \theta & 0 \\
0 & \sin \theta & \cos \theta & 0 \\
0 & 0 & 0 & 1
\end{array}\right], R_{y}=\left[\begin{array}{rrrr}
\cos \theta & 0 & \sin \theta & 0 \\
0 & 1 & 0 & 0 \\
-\sin \theta & 0 & \cos \theta & 0 \\
0 & 0 & 0 & 1
\end{array}\right], R_{z}=\left[\begin{array}{rrrr}
\cos \theta & -\sin \theta & 0 & 0 \\
\sin \theta & \cos \theta & 0 & 0 \\
0 & 0 & 1 & 0 \\
0 & 0 & 0 & 1
\end{array}\right]
\end{aligned}
$$

$H$ is the shear components and given by:-

$$
H=\left[\begin{array}{cccc}
1 & h_{x y} & h_{x z} & 0 \\
h_{y x} & 1 & h_{y z} & 0 \\
h_{z x} & h_{z y} & 1 & 0 \\
0 & 0 & 0 & 1
\end{array}\right]
$$

$S$ is the scale components and given by:-

$$
S=\left[\begin{array}{cccc}
s_{x} & 0 & 0 & 0 \\
0 & s_{y} & 0 & 0 \\
0 & 0 & s_{z} & 0 \\
0 & 0 & 0 & 1
\end{array}\right]
$$

\section{Local Alignment}

After the 3D affine registration, a local registration technique has been proposed to compensate for local lung motion and deformations between successive CT scans. In order to avoid problems associated with intensity variations between the scans, the proposed approach exploits geometric features, rather than using only image intensities, to deform each voxel in the source image to its matching or correspondence in the target image. These geometric features are estimated from the calculated field vectors by solving the 3D Laplace equation between each corresponding iso-surfaces in the segmented lung objects: 


$$
\nabla^{2} \gamma=\frac{\partial^{2} \gamma}{\partial x^{2}}+\frac{\partial^{2} \gamma}{\partial y^{2}}+\frac{\partial^{2} \gamma}{\partial z^{2}}=0
$$

where $\gamma(x, y, z)$ is the calculated potential between the reference and target surfaces, which results in intermediate equipotential surfaces and streamlines, being everywhere orthogonal to all equipotential surfaces and establishes natural voxel correspondences between the surfaces. In medical imaging, Laplace-equation based approaches have been previously used for colon surface flattening, centerline extraction [397], and thickness measurements $[398,399]$. To the best of our knowledge, the integration between Laplace and GGMRF is the first of its kind to be introduced in 2D/3D local registration.

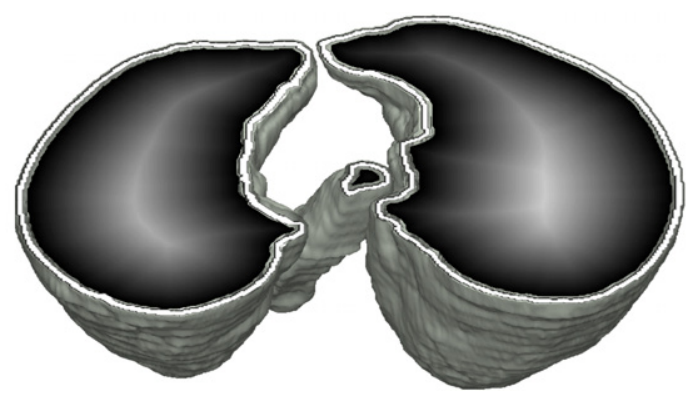

(a)

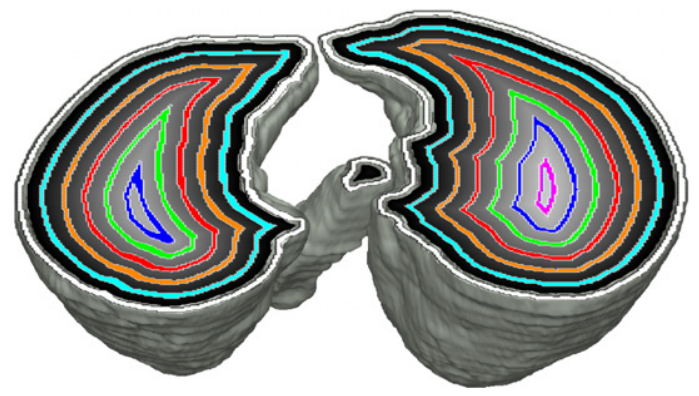

(c)

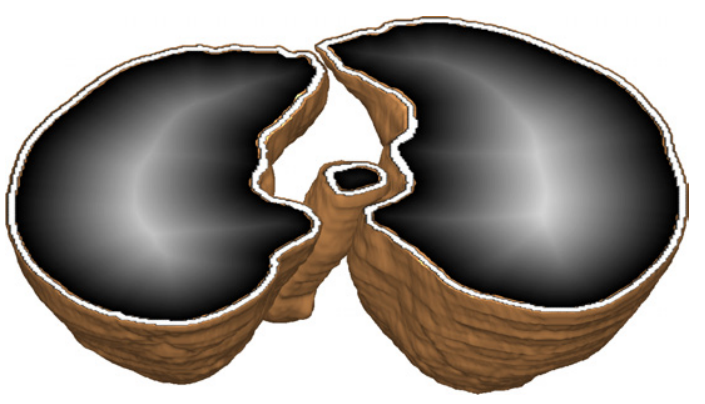

(b)

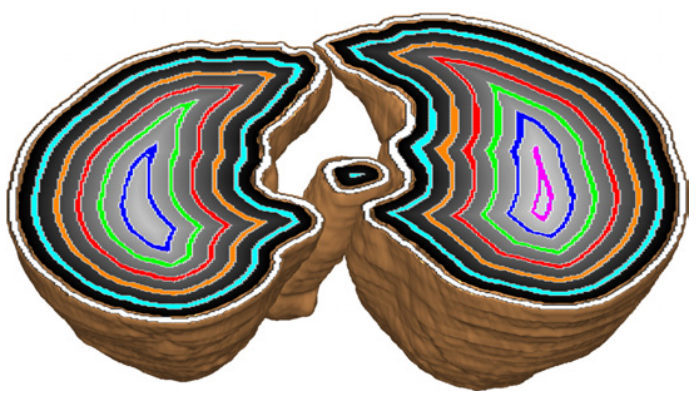

(d)

FIGURE 33: Iso-surfaces generation: the reference and target distance maps $(a, b)$, and their iso-surfaces $(c, d)$.

The proposed Laplace-based registration is based on deforming each voxel of segmented lung objects over a set of nested iso-surfaces, which is generated for both the target and reference objects. To find the iso-surfaces, a distance map is generated inside the seg- 


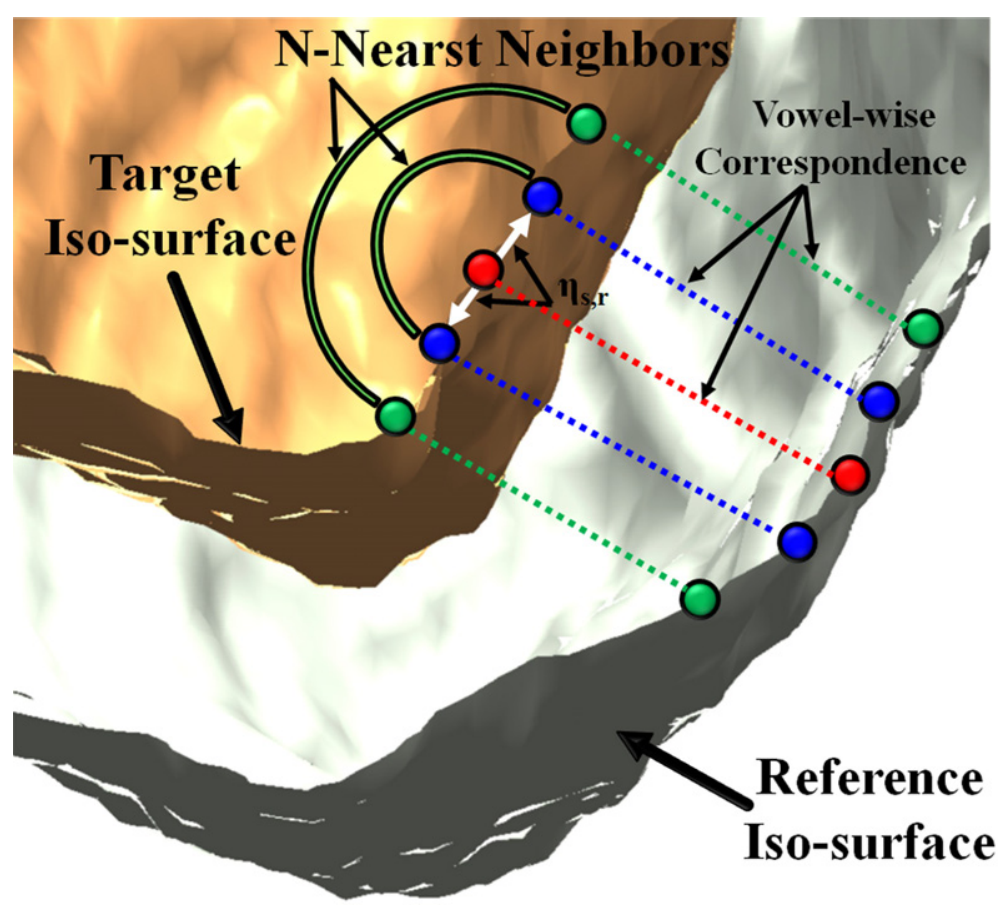

FIGURE 34: Schematic illustration of the N-nearest voxels.

mented lungs (Figure 33 (a,b)) using the fast marching level set [396]. Then, a collection of iso-surfaces within the lungs is formed, which is generated at equal distances from the lung borders (Figure $33(\mathrm{c}, \mathrm{d})$ ). The spacing between the iso-surfaces within each segmented lung is adjusted such that the final number of iso-surfaces for both the reference and target lungs is the same. Subsequently, the Laplace equation is applied to the intersection between corresponding reference and target iso-surfaces to co-locate their corresponding voxels. In order to estimate $\gamma(x, y, z)$, a second-order central difference method and the iterative Jacobi approach has been used with the high potential being applied for the target iso-surface while the low potential for the reference iso-surfaces:- 


$$
\begin{array}{r}
\gamma^{i+1}(x, y, z)=\frac{1}{6}\left\{\gamma^{i}(x+\Delta x, y, z)+\gamma^{i}(x-\Delta x, y, z)+\gamma^{i}(x, y+\Delta y, z)+\gamma^{i}(x, y-\Delta y, z)+\right. \\
\left.\gamma^{i}(x, y, z+\Delta z)+\gamma^{i}(x, y, z-\Delta z)\right\}
\end{array}
$$

where $\gamma^{i}(x, y, z)$ is the estimated potential at $(x, y, z)$ during the $i^{\text {th }}$ iteration; and $\Delta x, \Delta y$, and $\Delta z$ are the step length or resolution in $x, y$ and $z$ directions, respectively. Algorithm 6 summarizes basic steps for the co-allocation of voxel-wise correspondences using the Laplace equation.

Algorithm 6 Solution of the Laplace Equation Between two Surfaces

1 Step:1 Find the surface of both target and reference objects.

2 Step:2 Initial condition: Set the maximum and minimum potential $\gamma$ at the target surface and the corresponding reference surface, respectively.

3 Step:3 Estimate $\gamma$ between both iso-surfaces using Equation (22).

4 Step:5 Iterate Step 3 until convergence is achieved (i.e., there is no change in the estimated $\gamma$ values between iterations).

\section{Correspondences Refinement}

In order to avoid any anatomical distortions that result from solving the Laplace equation, another smoothness constraint (regularization) is employed to preserve the lung anatomy. The proposed constraint preserves the relative position between the neighboring voxels on the target image before and after displacements through iterative energy minimization using a 3D GGMRF model [400] on the initial reference-target iso-surfaces voxel-wise correspondences. Also, this constraint preserves the visual appearance matching between the reference and the target neighboring voxels. For each voxel on the target iso-surfaces, its correspondence on the reference iso-surfaces is iteratively refined by 
a GGMRF model [400] using the voxels neighborhood system ( $N$-nearest neighbors, Figure 34) using their maximum A posteriori (MAP) estimates [400] and voxel-wise stochastic relaxation (iterative conditional mode (ICM)) that jointly optimize $x, y$, and $z$ spatial coordinates using the model in [401]). Each voxel correspondence is refined using the 3D GGMRF model given in Equation (23) as follow:

$$
\begin{aligned}
\widehat{\mathbf{p}_{\mathbf{s}}}=\arg \min _{\widetilde{\mathbf{p}_{\mathbf{s}}}=\left({\widetilde{x_{s}}}^{r e f},{\widetilde{y_{s}}}^{r e f},{\widetilde{z_{s}}}^{r e f}\right)}\left\{\left(\left|x_{s}^{r e f}-{\widetilde{x_{s}}}^{r e f}\right|^{\alpha}+\left|y_{s}^{r e f}-{\widetilde{y_{s}}}^{r e f}\right|^{\alpha}+\left|z_{s}^{r e f}-{\widetilde{z_{s}}}^{r e f}\right|^{\alpha}\right)\right. \\
+\rho^{\alpha} \lambda^{\beta} \sum_{r \in N} \eta_{s, r}\left(\left|{\widetilde{x_{s}}}^{r e f}-x_{r}^{r e f}\right|^{\beta}+\left|{\widetilde{y_{s}}}^{r e f}-y_{r}^{r e f}\right|^{\beta}+\left|\widetilde{z}_{s}^{r e f}-z_{r}^{r e f}\right|^{\beta}\right) \\
\left.+\left|q_{s^{\prime}}{ }^{\text {tar }}-{\widetilde{q_{s}}}^{r e f}\right|^{\alpha}+\rho^{\alpha} \lambda^{\beta} \sum_{r \in N} \eta_{s, r}\left|\widetilde{q}_{s^{\prime}}^{\text {tar }}-q_{r}^{r e f}\right|^{\beta}\right\}
\end{aligned}
$$

where $\mathbf{p}_{\mathbf{s}}=\left(x_{s}^{r e f}, y_{s}^{r e f}, z_{s}^{r e f}\right)$ and $\widetilde{\mathbf{p}_{\mathbf{s}}}=\left({\widetilde{x_{s}}}^{r e f},{\widetilde{y_{s}}}^{\text {ref }},{\widetilde{z_{s}}}^{\text {ref }}\right)$ denote the initial 3D locations of the target voxels' correspondences and their expected estimates on the reference; $q_{s^{\prime}}{ }^{t a r}$ and $\widetilde{q}_{s}^{r e f}$ are the target voxel intensity and its estimate correspondences on the reference, respectively; $N$ is the number of the nearest neighbor voxels (Figure 34); $\eta_{s, r}$ is the GGMRF potential, and $\rho$ and $\lambda$ are scaling factors. The parameter $\beta \in[1.01,2.0]$ controls the level of smoothing (e.g., $\beta=2$ for smooth vs. $\beta=1.01$ for relatively abrupt edges). The parameter $\alpha \in\{1,2\}$ determines the Gaussian, $\alpha=2$, or Laplace, $\alpha=1$, prior distribution of the estimator. the experiments below were conducted with $\rho=1, \lambda=5, \beta=1.01, \alpha=2$, and $\eta_{s, r}=\sqrt{2}$ for all directions. The final deformation field that maps each voxel on the target to its corresponding voxel in the reference is calculated based on both global alignment and local (non-rigid) alignment using laplace-based solution with GGMRF regularization. A typical example for finding the voxel-to-voxel correspondences between the reference and target iso-surfaces, before and after GGMRF refinement is shown in Figure 35, obtained using basic steps of the proposed registration methodology in Algorithm 7. It is clear in 
Figure 35 that the voxels' correspondences before applying the GGMRF regularization have some intersections, specially in the concavity regions, that contradicts the anatomy preservation. These distortions are solved by applying GGMRF, Equation (23), as it ensures that each voxel is deformed to the target's voxel that minimizes the transition energy between correspondences' intensity values.

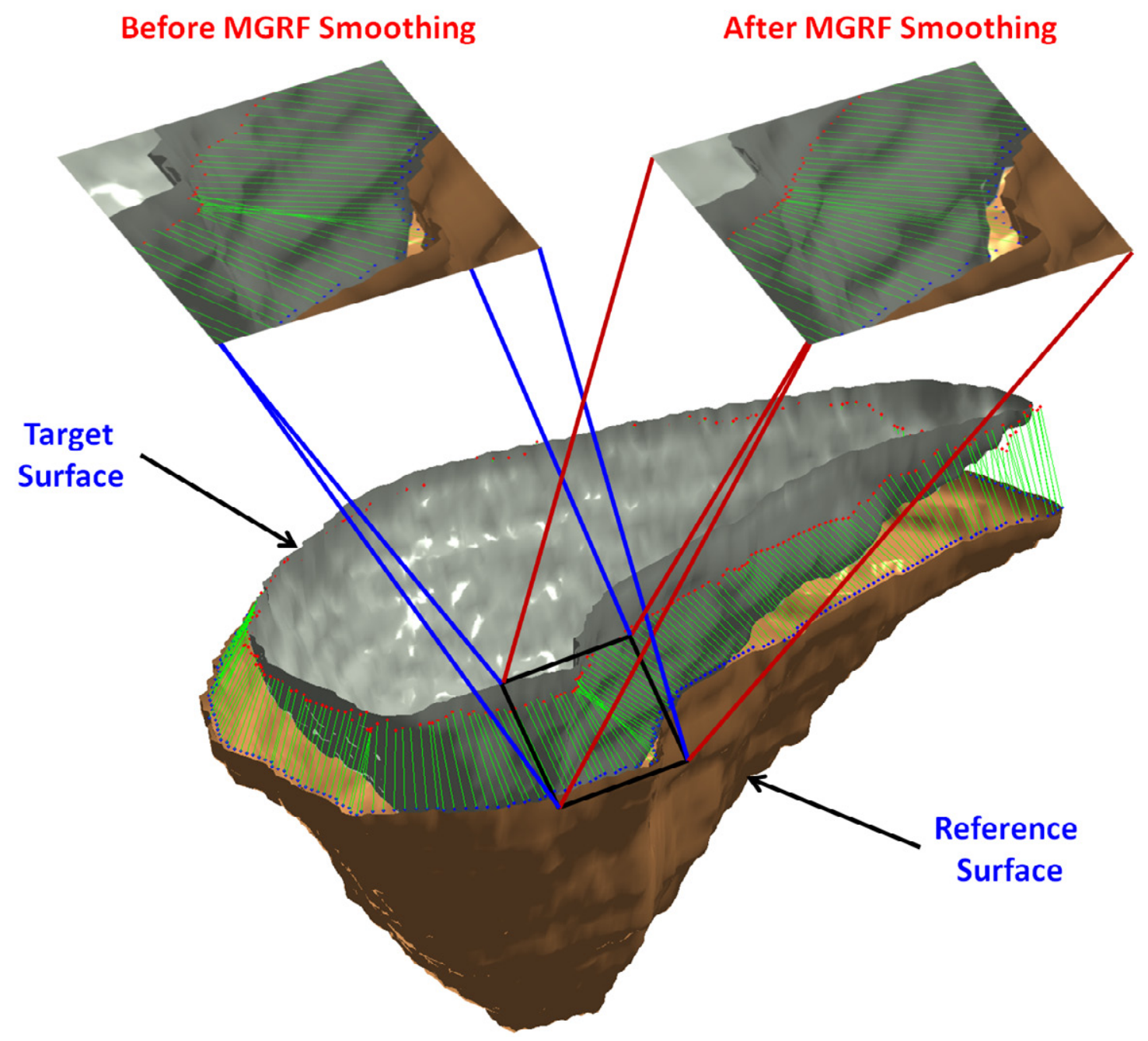

FIGURE 35: GGMRF spatial refinement effect on the voxel-wise correspondences between two iso-surfaces. 
\begin{tabular}{l}
\hline Algorithm 7 Proposed Registration Methodology \\
\hline - Global Alignment
\end{tabular}

1. Step:1b Generate the 3D distance maps inside the segmented lungs (Figure 33(a,b)) using fast marching level sets [396].

2. Step:2b Globally align the generated distance maps using a 3D affine registration and MI [395] as a similarity measure.

\section{- Local Alignment}

1. Step:3b Generate the nested iso-surfaces for the target and reference maps (Figure $33(c, d)$ ).

2. Step:4b Use Algorithm 6 to solve Laplace equation between corresponding isosurfaces.

3. Step:5b Compute components of the gradient vectors in X-, Y-, and Z-directions for the estimated potential in Step $4 \mathrm{~b}$.

4. Step:6b Form the streamlines using the gradient vectors in Step 5b, then find the voxelwise correspondences between the iso-surfaces matched by forming the streamlines.

5. Repeat Steps $4 b-6 b$ for the next set of the corresponding iso-surfaces.

- Step:8b Refinement: Use the 3D GGMRF model, Equation (23), to refine the correspondences.

\section{Experimental Results and Validation}

The proposed registration methodology has been tested on the clinical datasets that were collected from 25 patients at the end-expiration breath hold (EE-BH). Each patient has two low dose CT (LDCT) scans, with a three-month period between each two successive scans. This clinical database was collected by the LDCT scan protocol using a multidetector GE Light Speed Plus scanner (General Electric, Milwuakee, USA) with the following scanning parameters: the slice thickness of $2.5 \mathrm{~mm}$ reconstructed every $1.5 \mathrm{~mm}$; scanning pitch $1.5 \mathrm{~mm} ; 140 \mathrm{KV} ; 100 \mathrm{MA}$; and the field-of-view $36 \mathrm{~cm}$. 
(a)
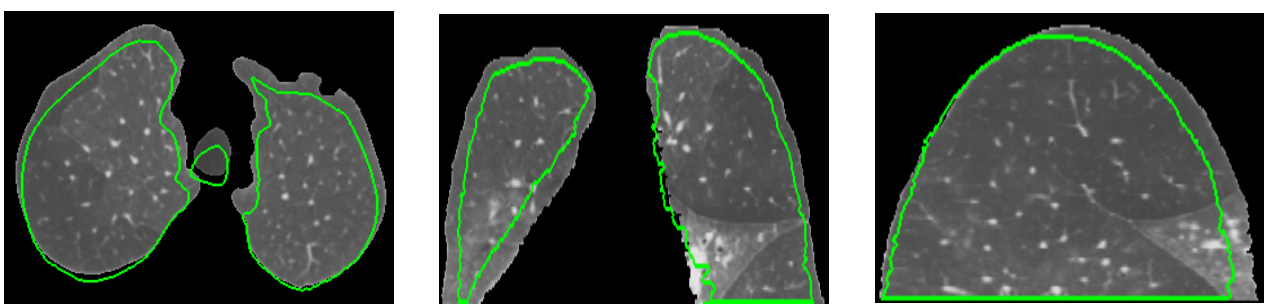

(b)
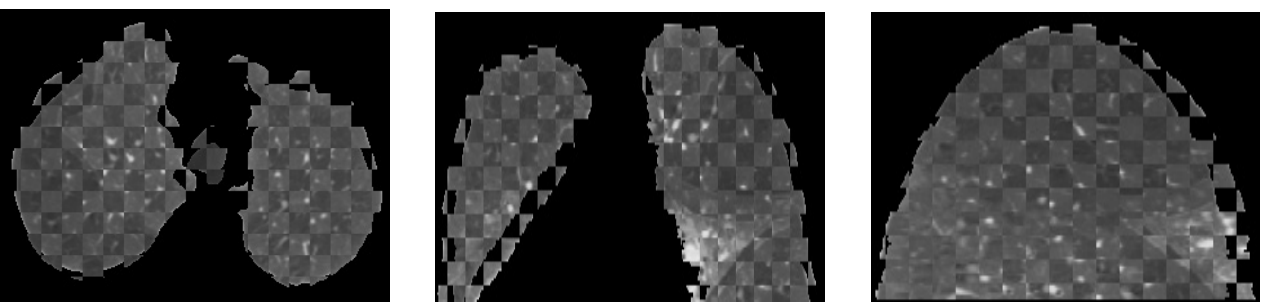

(c)
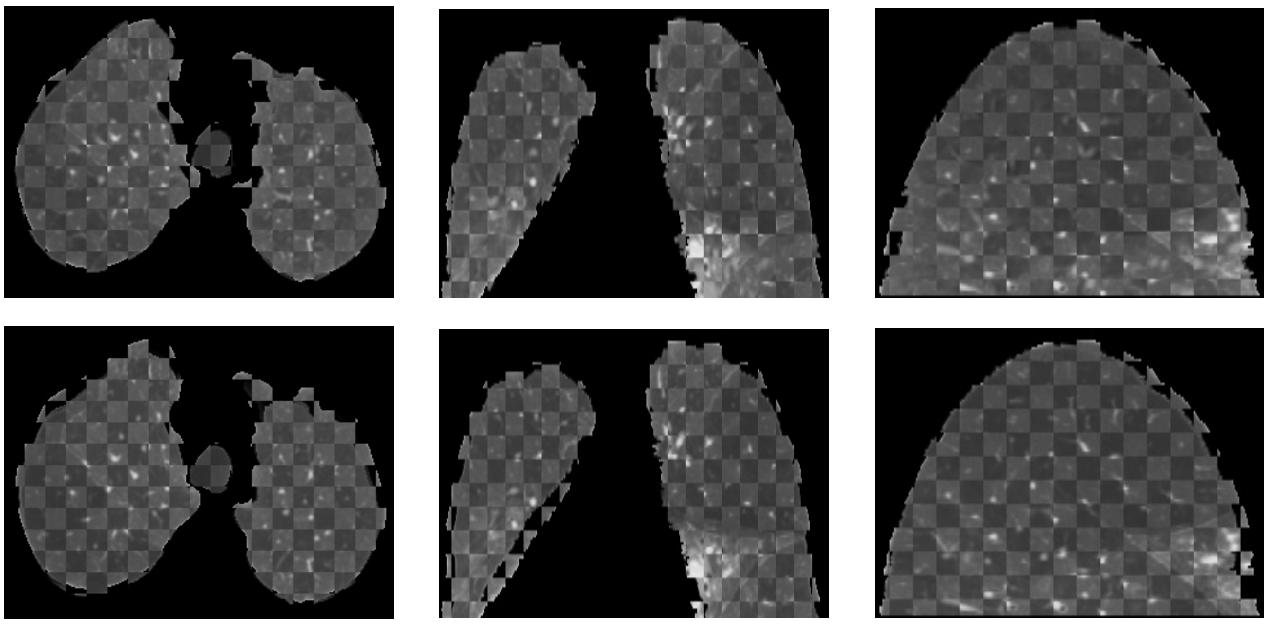

(d)

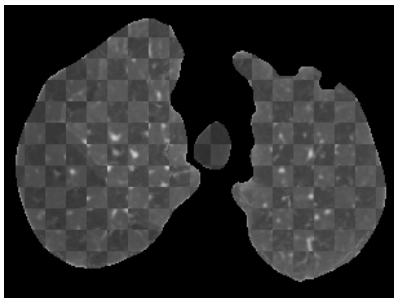

(A)

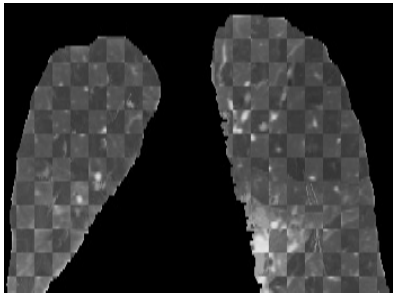

(C)

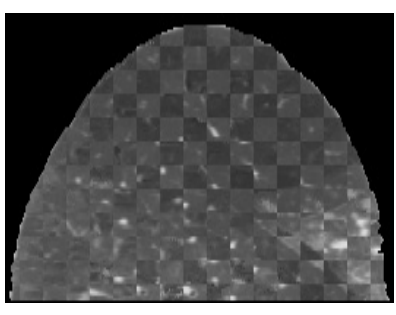

$(\mathrm{S})$

FIGURE 36: 3D global and local registration results projected into axial, coronal, and sagittal planes: (a) reference objects with overlaid target edges (green); and the superimposed checkerboard visualization (b) before global alignment (SSD =0.032), (c) after global alignment $(\mathrm{SSD}=0.024),(\mathrm{d})$ after FFD alignment $(\mathrm{SSD}=0.020)$, and $(\mathrm{d})$ after the iso-surfaces-alignment $(\mathrm{SSD}=0.015)$. 


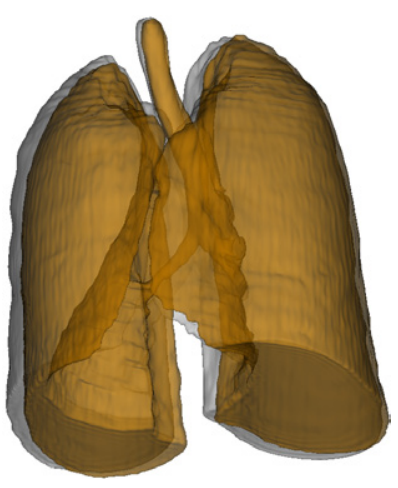

(a)

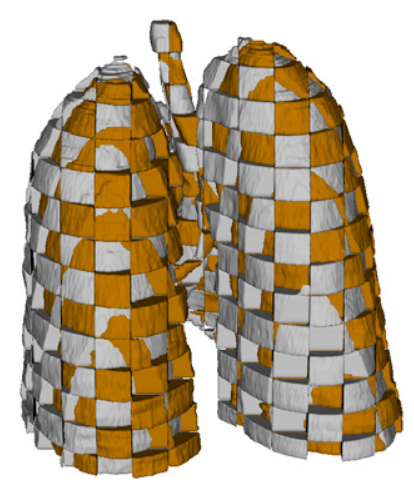

(b)

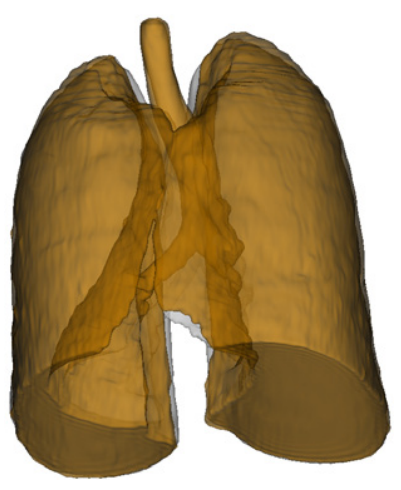

(c)

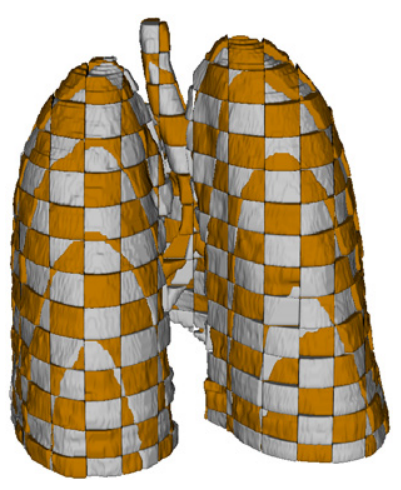

(d)

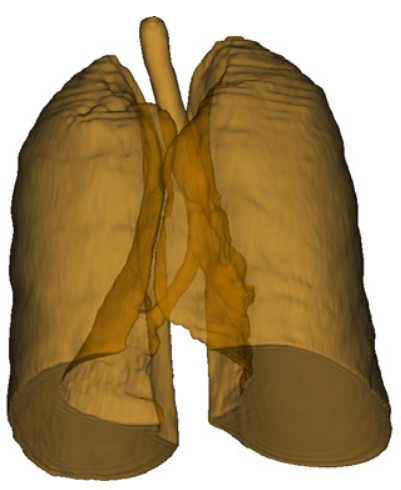

(e)

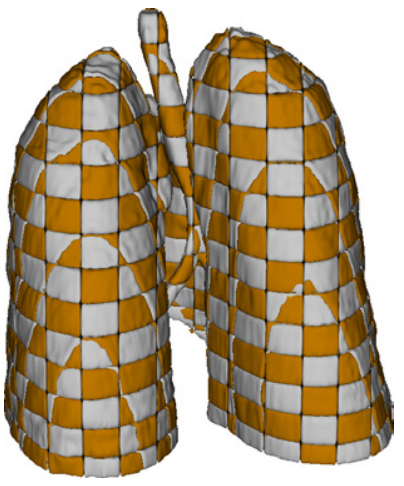

(f)

FIGURE 37: 3D registration results: superimposed reference and target CT data before global alignment(a), after global alignment(c), after the iso-surfaces-alignment(e). and their respective superimposed checkerboard visualization(b), (d), and (f). Note that the reference and target objects are shown in grey and orange colors, respectively. 


\section{Qualitative Evaluation}

To qualitatively assess the accuracy of the approach, 3D registration results for one subject from a locally-acquired data is shown in Figure 36. For visual assessment of the goodness of registration, the checkerboard displays has been used, which use the net contour as a metric of the agreement between the two registered objects. As readily seen, the global alignment in Figure 36 (f, second row) increases the area of overlap between the lung objects. However, there is still misalignment between the surfaces, which is effectively reduced after applying the proposed Laplace-based nonrigid registration with the GGMRF smoothness constraints (see Figure 36 (f, third row)). To highlight the advantage of the proposed approach, it has been compared with the 3D free-form-deformations (FFD) registration approach [402] using SSD as shown in Figure 36 caption.

\section{Quantitative Evaluation}

For quantitative assessment of the proposed local registration method, it has been tested on both in-vivo and simulated data using the target registration error (TRE) for tracked bifurcation points before and after the registration. TRE is calculated as the Euclidean distance between landmarks as defined by Equation (24).

$$
\operatorname{TRE}(i)=\sqrt{\left(x_{i, 1}-x_{i, 2}\right)^{2}+\left(y_{i, 1}-y_{i, 2}\right)^{2}+\left(z_{i, 1}-z_{i, 2}\right)^{2}}
$$

Where $\left(x_{i, 1}, y_{i, 1}, z_{i, 1}\right)$ and $\left(x_{i, 2}, y_{i, 2}, z_{i, 2}\right)$ represent the Euclidean $3 D$ coordinates for the $i$ th pair of matching landmarks.

a. In-vivo Data Validation To validate the proposed approached, two different databases has been used: a locally-acquired database and a pubblicaly available database with expert-annotated land mark correspondences between scan pairs [403].

First, a control study has been performed in which two scans has acquired from the same patient at the EE-BH using the scanning protocol described above. Thus, the only differ- 
ence between the two scans was that the patient was asked to make a global rotation and translation after the first scan. Scan \#1 before the patient's movement has been used as a reference image to register scan \#2 after the patient's movement. The average co-alignment errors are $11.60 \pm 5.30 \mathrm{~mm}$, and $1.40 \pm 0.7 \mathrm{~mm}$ before and after registration respectively, based on calculating the Euclidean distance between 250 landmark points manually selected by a radiologist on the reference and the registered target data.

Secondly, evaluation of the proposed local registration method was also performed on a publically available database, DIR database [403]. This database provides 4D-CT respiratory sequences with manually selected landmarks. Each 4D-CT subject consists of ten different breathing phase with a typical size of $256 \times 256 \times 100$ voxels and an average resolution of $1 \times 1 \times 1 \mathrm{~mm}^{3}$. The average TRE is calculated, for each subject of this database, for 300 (distributed through the lungs) manually selected landmarks between the images of the extreme inhale and extreme exhale phases. More details about the datasets can be found in [403]. Displacement error before and after registration for the proposed approach compared with Heinrich et al. approach [393] are summarized in Table 15. This results demonstrate the ability of the proposed approach to accurately register the lung tissues and reduce the displacement error compared to one of the state-of-the-art techniques.

b. Simulated Data Validation Due to the inter-observer variability and handshaking errors in selecting matched points, the proposed approach has been validated using simulated local deformations that have been applied for real CT data. Namely, local deformations has been simulated on the real LDCT dataset by using a frequently employed method [404] to apply a known local transformation to the test dataset and register the original scan with the result. Three different types of the deformation fields has been generated; small, moderate, and large deformations; indicated in Table 16. the proposed registration model has been applied to each type of deformation, and the accuracy has been assessed quantitatively by comparison of the simulated and recovered voxel displacements. Figure 38 shows typical results of such comparisons for a set of 1,309 randomly selected 
TABLE 15: Comparison of registration accuracy for the 4D-CT DIR-lab data (All units are in $\mathrm{mm}$ ).

\begin{tabular}{|c|c|c|c|}
\hline \multirow{3}{*}{ Subj } & & \multicolumn{2}{|c|}{$\begin{array}{l}\text { Displacement After } \\
\text { Registration }\end{array}$} \\
\hline & \multicolumn{3}{|c|}{\begin{tabular}{|l|l|l} 
Registration & Froposed approacn & nemrncn el al. [395]
\end{tabular}} \\
\hline & Mean \pm std & Mean \pm std & Mean \pm std \\
\hline 1 & $3.89 \pm 2.78$ & $0.96 \pm 0.60$ & $0.97 \pm 0.50$ \\
\hline 2 & $4.34 \pm 3.90$ & $0.89 \pm 0.60$ & $0.96 \pm 0.50$ \\
\hline 3 & $6.94 \pm 4.05$ & $1.63 \pm 0.96$ & $1.21 \pm 0.7$ \\
\hline 4 & $9.83 \pm 4.86$ & $1.92 \pm 1.56$ & $1.39 \pm 1.0$ \\
\hline 5 & $7.48 \pm 5.51$ & $1.47 \pm 1.07$ & $1.72 \pm 1.6$ \\
\hline 6 & $10.89 \pm 6.97$ & $1.82 \pm 1.29$ & $1.49 \pm 1.0$ \\
\hline 7 & $11.28 \pm 7.33$ & $1.29 \pm 0.78$ & $1.58 \pm 1.2$ \\
\hline 8 & $14.99 \pm 9.01$ & $1.57 \pm 1.12$ & $2.11 \pm 2.4$ \\
\hline 9 & $7.91 \pm 3.98$ & $1.07 \pm 0.54$ & $1.36 \pm 0.7$ \\
\hline 10 & $7.30 \pm 6.35$ & $1.05 \pm 0.75$ & $1.55 \pm 1.6$ \\
\hline \multicolumn{2}{|r|}{ Overall average } & $1.37 \pm 1.03$ & $1.44 \pm 0.97$ \\
\hline \multicolumn{2}{|r|}{$\mathrm{P}$-value } & \multicolumn{2}{|c|}{0.0095} \\
\hline
\end{tabular}

voxel positions from the lung vascular system.

\section{Summary}

This chapter introduced a novel registration methodology to co-align successive 3D CT scans of a segmented lung object with a given prototype. The proposed approach involves both global (based on 3D affine registration) and local (based on the solution of 


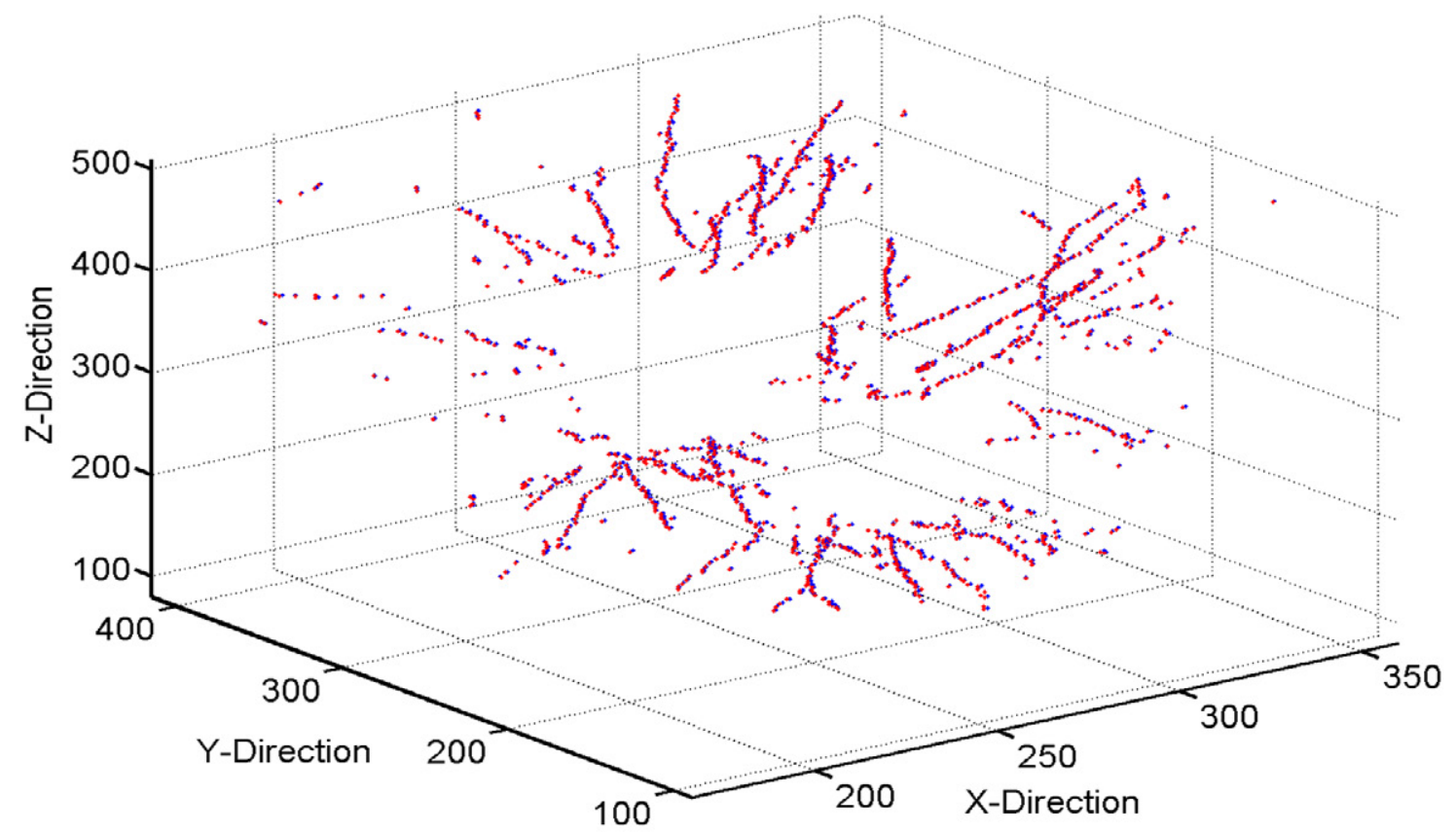

FIGURE 38: Comparing the 1,309 randomly selected voxel positions from the lung vascular system for a large deformation field: The ground truth is in blue, and the corresponding positions found with the proposed method are in red. Note that all units are in $\mathrm{mm}$ in all directions.

TABLE 16: Registration accuracy for simulated displacements (All units are in $\mathrm{mm}$ ).

\begin{tabular}{|c|c|c|c|c|c|c|}
\cline { 2 - 7 } \multicolumn{1}{c|}{} & \multicolumn{3}{c|}{$\begin{array}{c}\text { Simulated } \\
\text { Displacement }\end{array}$} & \multicolumn{3}{c|}{ Alignment } \\
\cline { 2 - 7 } & Max & Mean & SD & Max & Mean & SD \\
\hline Small & 1.7 & 0.6 & 0.4 & 0.6 & 0.4 & 0.3 \\
\hline Moderate & 10.8 & 2.3 & 0.7 & 1.4 & 1.0 & 0.4 \\
\hline Large & 19.9 & 9.1 & 1.1 & 2.1 & 1.2 & 1.6 \\
\hline
\end{tabular}

the Laplace equation) alignment steps. the proposed local alignment step possesses the ability to accurately co-allocate voxel-on-voxel matches between the target and reference lung objects, which is followed by a smoothness constraint that preserves lung anatomy 
(both lung geometry and its visual appearance). In-vivo and simulated data validation results confirm that lung registration based on the proposed methodology is more robust and accurate than popular conventional algorithms. The work presented in this chapter has been published in the International Symposium on Biomedical Imaging (ISBI) conference [405]. 


\section{CHAPTER V \\ COMPUTER AIDED DIAGNOSIS SYSTEM FOR ACCURATE DETECTION OF RADIATION-INDUCED LUNG INJURY}

A novel framework for identifying radiation-induced lung injury (RILI) after radiation therapy (RT) using 4D computed tomography (CT) scans is introduced in this chapter. After deformable image registration, the framework segments lung fields, extracts functional and textural features, and classifies lung tissues. The deformable registration locally aligns consecutive phases of the respiratory cycle using gradient descent minimization of the conventional dissimilarity metric (the sum of squared signal differences). Then an adaptive shape prior, a first-order intensity model, and a second-order lung tissues homogeneity descriptor are integrated to segment the lung fields. In addition to common lung functionality features, such as ventilation and elasticity, specific regional textural features are estimated by modeling the segmented images as samples of a novel $7^{\text {th }}$-order contrastoffset-invariant Markov-Gibbs random field (MGRF). Finally, a random forest (RF) classifier is applied to distinguish between the injured and normal lung tissues. The proposed framework has been tested on CT data sets from 13 patients who had undergone the RT treatment. Experimental results demonstrate the proposed framework shows promise for building a CAD system for early RILI detection.

\section{A. Introduction}

Radiation-induced lung injury (RILI) is the main side effect of radiation therapy (RT) for lung cancer patients. Although higher radiation doses increase the RT effectiveness for tumor control, this can lead to lung injury because a greater quantity of normal lung tissue is included in treated areas. From almost $13 \%$ to $37 \%$ of patients who under- 
went the RT developed lung injuries following treatment [406]. The RILI severity ranges from ground-glass opacities and consolidation at the early phase to fibrosis and traction bronchiectasis in the late phase. Early detection of lung injury will help to improve man-

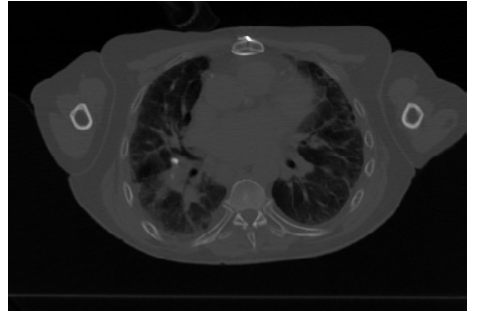

(a)

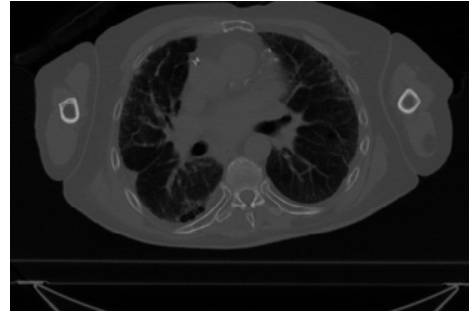

(b)

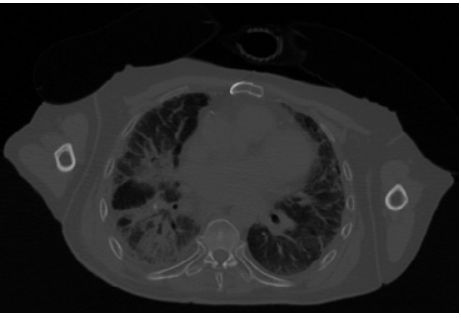

(c)

FIGURE 39: Effect of radiation on normal lung tissues; three axial slices from Pretreatment scan (a), 3 months follow-up scan (b), and 6 months follow-up scan (c). Note that they are cropped from the same center coordinate to show the effect of global and local misalignment.

agement of the treatment. Relying on only the CT appearance, i.e., Hounsfield units (HU), leads to a late RILI detection that will make the treatment more difficult. As an alternative, detecting the early RILI development through monitoring lung functionality and lung texture changes may substantially improve the disease management. Although global pulmonary function tests (PFT), such as spirometry, measure an airflow obstruction/restriction, no regional lung function information is obtained. Alternatively, the lung functionality can be locally evaluated using nuclear imaging, e.g., SPECT ventilation and perfusion (V/Q) images. However, SPECT image acquisition is highly expensive and relatively slow machines, and the images have too low spatial resolution.

Recently, four-dimensional computed tomography (4D-CT) scans have gained attention for assessing lung functionality due to their high spatial resolution, faster acquisition, and low cost. Moreover, in addition to texture, many functional features can be derived from the 4D-CT scans. The lung ventilation can be derived from the 4D-CT scans and either these results are correlated with the SPECT (V/Q) scans, or the ventilation maps 
are correlated directly with certain clinical findings [407]. Latifi et al. [407] showed that the lung ventilation maps based on the 4D-CT correlate with 3D radiation dose distributions and suggested that these maps can be used in treatment planning to spare functional lung volumes. Yamamoto et al. [408] explored physiological validation, where the 4D-CT ventilation and V/Q SPECT images were compared by using HU changes and Jacobian determinant metrics. This comparison resulted in a much higher correlation between the 4D-CT ventilation and SPECT perfusion, leading to a conclusion that 4D-CT ventilation imaging has the potential to achieve high physiological accuracy. Reinhardt et al. [409] estimated local lung expansion from multiple respiratory CT images and showed that the Jacobian of the registration displacement field, directly relates to a specific volume change. Vinogradskiy et al. [410] correlated the lung dose and 4D-CT ventilation-based function to thoracic toxicity after the RT and assessed the ability of the dose volume and ventilation-based dose function metrics to predict for severe (grade $3+$ ) radiation pneumonitis (RP). In order to detect RILI, Zhang et al. [411] assessed early changes in the lung perfusion SPECT scans and PFT before and after intensity modulated radiotherapy (IMRT) for patients having locally advanced non-small cell lung cancer. The lung perfusion index (LPI) was calculated after quantification of the SPECT scans. A quantitative study by Cunliffe et al. [355] measured lung reactions to the RT and assessed the RP development by extracting intensity-based information to distinguish between the RP and non-RP patients. Sharifi et al [412] presented a prediction model for radiation-induced lung damage (RILD) by measuring only the lung tissue density changes per voxel. They found a correlation between the RT dose delivered to lung parts and the density changes over the time. Negahdar et al. [413] proposed the use of the strain to measure the regional deformation in the lung. They computed the lung deformation using their developed 3-D optical flow technique [414-416]. They compared the the calculated strain with Jacobian of the deformation fields using seven subjects with non-small cell lung cancer [417], in which they statistically correlated the voxel-wise values of the Jacobian and the maximal principal strain to the ventilation and perfusion 
SPECT images. The results showed that a higher correlation between the strain and the SPECT images than the Jacobian one. However, accurate estimation of these well know metrics depends mainly of the accuracy of the calculated deformation fields that should ensure accurate mapping.

In summary, the known methods for detecting the RT effects have several limitations. The global PFTs measure the total airflow and provide no information about regional functionality. Nuclear imaging based detection of defects in local pulmonary functions suffers from low spatial resolution. Conventional voxel-wise descriptors of lungs appearance are too sensitive to noise and take no account of dependences between adjacent voxels to suppress noise impacts. Common computational models for aligning the 4D-CT images do not guarantee proper voxel-to-voxel matches, and this may lead to inaccurate estimates of lung functionality parameters.

This chapter proposes a new and efficient computational framework to accurately align and segment lung regions from the 4D-CT images; extract discriminative features for the early RILI detection, and perform the detection.

\section{B. Methods}

The presented framework, Figure 40, takes the 4D-CT scans for the patient as an input, then process these input scans to extract physiological biomarkers that used for the identification of the RILI as an output. The processing pipeline for the biomarkers extraction and RILI detection is shown in Figure 41. The pipeline performs sequentially a non-rigid registration of the input 4D lung CT data; segments the lung fields with a newly developed image models and methodology; extracts functional and textural features, and detects the RILI by using a trainable random forest (RF) classifier of lungs tissues. The proposed framework is detailed below. 


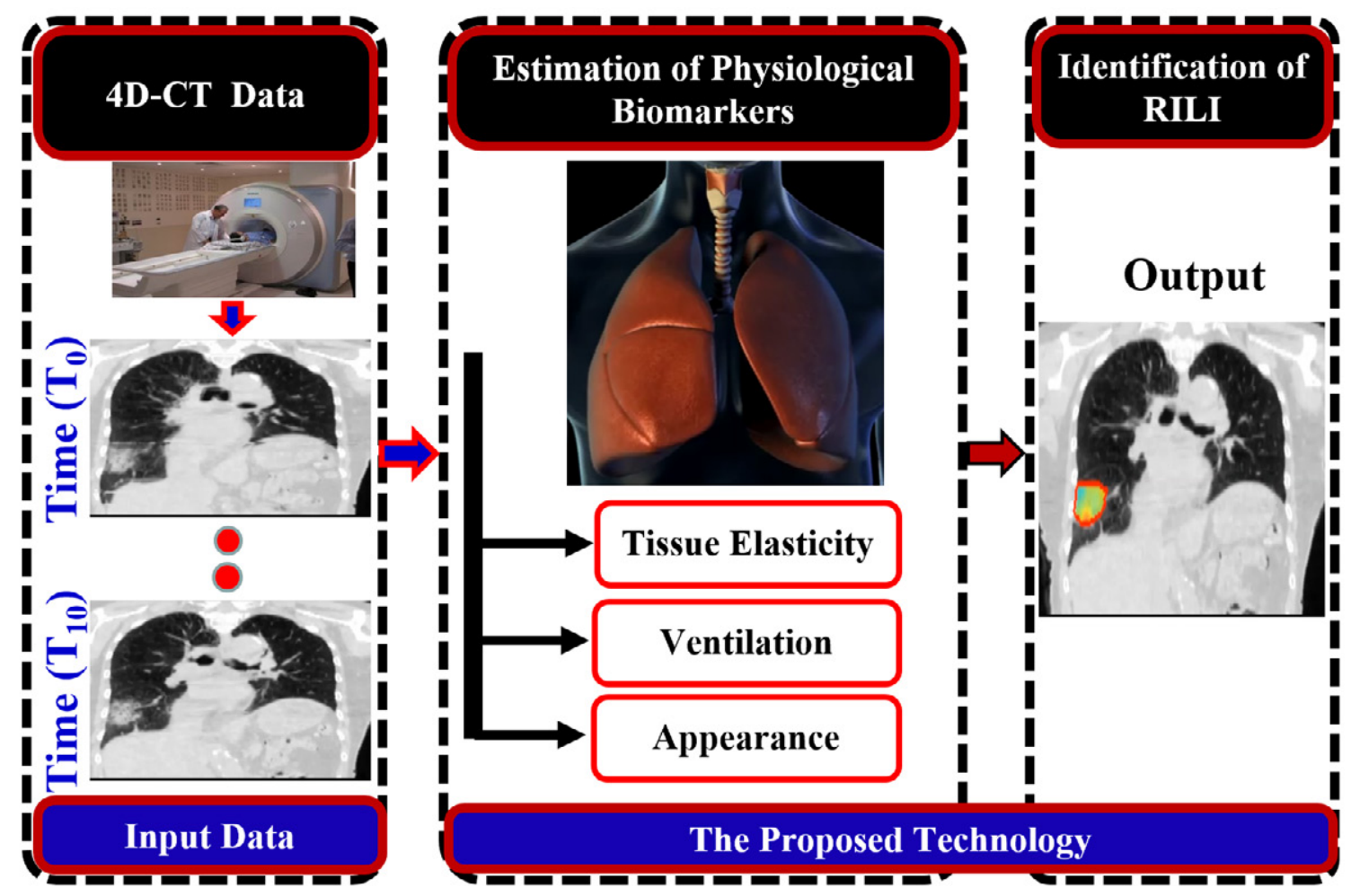

FIGURE 40: General framework for detecting the radiation-induced lung injuries (RILI).

\section{Deformable Image Registration}

Accurate estimation of regional functional features essentially requires accurate spatial mapping between successive pairs of 3D CT volumes of the respiratory cycle. Traditional 4D-CT registration tries to establish direct spatial correspondences between the peak exhale and peak inhale images. However, such a registration leads to sizeable potential errors in the displacement field due to large changes in lung volumes between the two peak phases. Therefore, any registration errors will greatly affect the estimated functionality features that depends on the deformation field to be calculated. To reduce these errors that might greatly affect the estimated features, a sequential deformable image registration has been performed between successive 3D-CT volumes of the respiratory cycle, see Figure 42. The registration establishes the voxel-wise displacement vector field $\mathbf{U}=\left\{\mathbf{u}(\mathbf{r})=\sum_{i=1}^{N-1} \mathbf{u}_{i}(\mathbf{r}): \mathbf{r} \in \mathbb{R}\right\}$, which integrates displacements between successive 


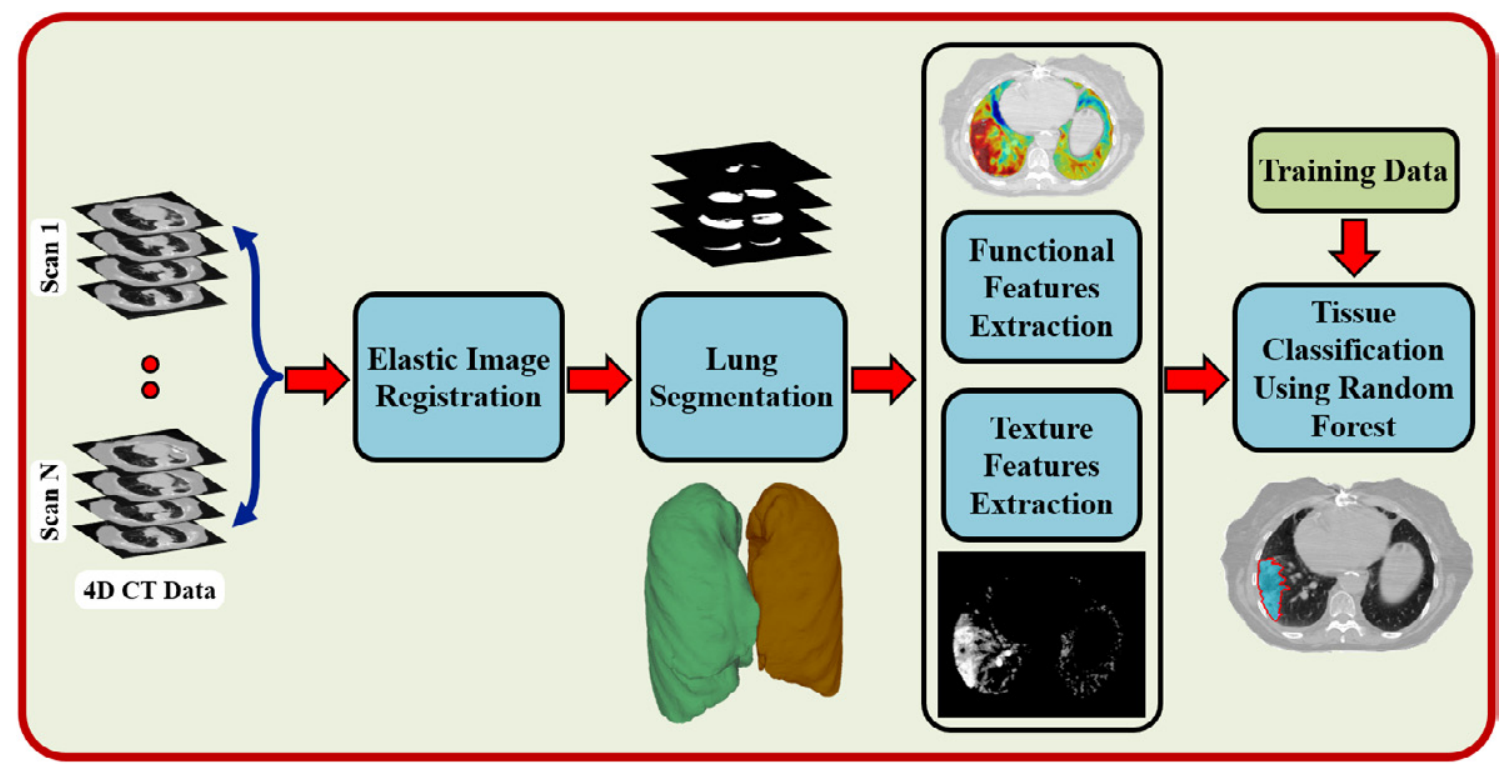

FIGURE 41: Processing pipeline framework for detecting the radiation-induced lung injuries (RILI).

3D-CT volumes from the peak exhale phase to the peak inhale phase. The total field, $U$, and its successive components, $\mathbf{U}_{i}=\left\{\mathbf{u}_{i}(\mathbf{r}): \mathbf{r} \in \mathbb{R}\right\}$, defined on the initial 3D-CT volume $\mathbb{R}$, determine gradual changes of image geometry and signals or features along the cycle.

The individual displacement fields are estimated by numerical minimization of a regularized registration error measured by the sum of squared differences. The regularization term, ensuring smoothness of the registration, accounts for physically elastic nature of lung tissue deformations over the respiratory cycle. This chapter uses the non-rigid registration approach proposed in Chapter IV that is base on global alignment using mutual information as a similarity measure to maximize the overlap between the $3 \mathrm{D}$ distance map for both the efference and target lung volumes followed by local alignment using the Laplace equation for correspondence allocation and GGMRF for regularization. 


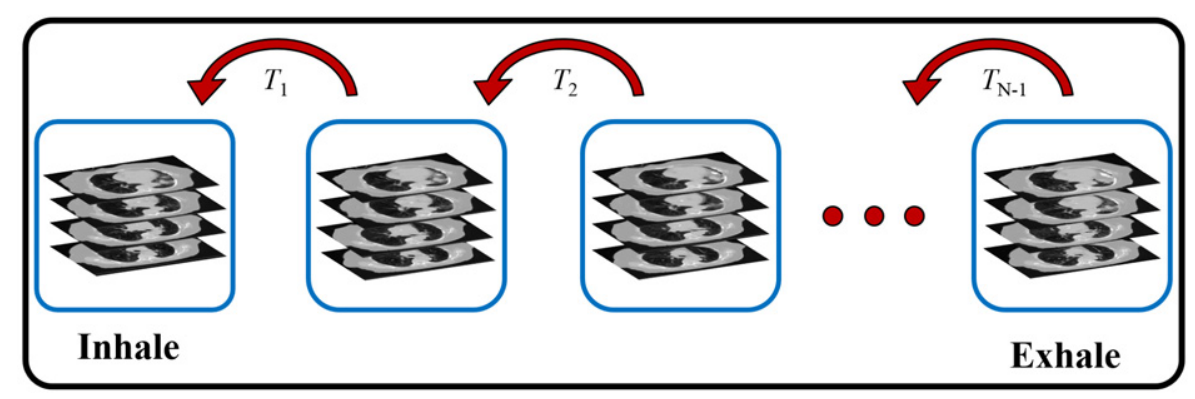

FIGURE 42: Successive 4D-CT data registration to obtain the final deformation field $\mathbf{u}(\mathbf{p})=\sum_{i=1}^{N-1} \mathbf{u}_{i}(\mathbf{p})$ between the peak inhale and peak exhale phases.

\section{4D-CT Lung Segmentation}

Segmented lungs' masks are a crucial component that will be used in all subsequent steps, either image analysis or classification. The lung fields are segmented in order to guide their analysis and ensure that all potentially injured areas will be examined. The segmentation reduces also inter-subject variations of estimated features due to normalizing these features inside the lung fields in each data set by averaging over its chest region (i.e., the chest acts as a normalizing volume). Moreover, the segmented lungs at different respiratory phases will be used in the non-rigid registration in the next step. In the final step, it will be used as an ROI for the classification using RF classifier. So accurate segmentation is a must for accurate results at each step. To segment the 4D-CT scans, some modifications has been added to the $3 \mathrm{D}$ accurate lungs segmentation framework, that has been introduced in Chapter III which has the ability to segment lungs with wide range of pathologies, to be able to segment the 4D-CT scans. After segmenting the Exhale phase, using the 3D segmentation [384], the segmentation labels is propagated to the subsequent phases using the modified adaptive shape prior component only without the need to any other components which leads to a an accurate and faster segmentation. Visual appearances of different respiratory phases images and the exhale images guide the shape prior adaptation as follows (outlined in Algorithm 8). Each voxel $\mathbf{r}$ of the different phase image $\mathbf{t}$ is mapped to the same location at the exhale lattice. To adapt the shape prior at each mapped location $\mathbf{r}$, 


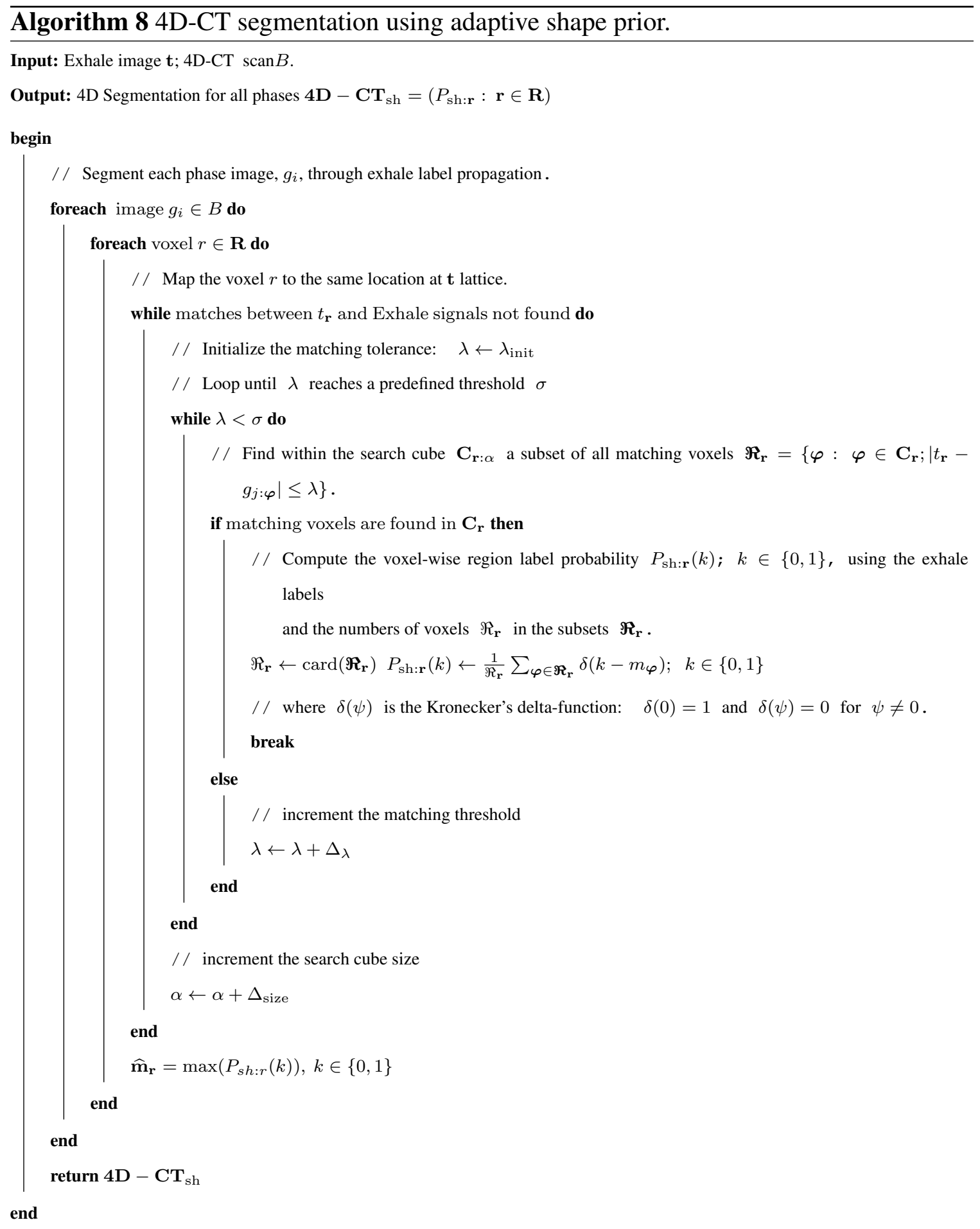


an initial search cube $\mathbf{C}_{\mathbf{r}}$ of size $n_{x: \mathrm{i}} \times n_{y: \mathrm{i}} \times n_{z: \mathrm{i}}$ is centered at the mapped location $\mathbf{r}$ for finding in the cube all the exhale voxels with signal deviations to within a predefined fixed range, $\lambda$, from the mapped input signal, $t_{\mathbf{r}}$. If such voxels are absent in the exhale, the cube size increases iteratively until the voxels within the predefined signal deviation range are found or the final cube size is reached $\left(n_{x: \mathrm{i}}=n_{y: \mathrm{i}}=n_{z: \mathrm{i}}=3 ; \lambda\right.$ from 50 to 125 with the step of $\Delta_{\lambda}=25$, and the final cube size of $n_{x: \mathrm{f}}=n_{y: \mathrm{f}}=n_{z: \mathrm{f}}=11$ were used in the experiments). Then the voxel-wise probabilities, $P_{\text {sh:r }}(k) ; k \in \mathbf{K}$, for the adaptive shape prior are estimated based on the found voxels of similar appearance and their labels. Let $\Re_{\mathbf{r}}=\left\{\boldsymbol{\varphi}: \varphi \in \mathbf{R} ; \boldsymbol{\varphi} \in \mathbf{C}_{\mathbf{r}} ;\left|g_{\varphi}-t_{\mathbf{r}}\right| \leq \lambda\right\}$ be a subset of similar voxels within the cube $\mathrm{C}_{\mathrm{r}}$ in the exhale image. Let $\Re_{\mathrm{r}}=\operatorname{card}\left(\Re_{\mathrm{r}}\right)$ denote the cardinality (number of voxels) of this subset; and $\delta(z)$ be the Kronecker's delta-function: $\delta(0)=1$ and 0 otherwise. The final probability for each voxel calculated as:

$$
P_{\text {sh:r }}(k)=\frac{1}{\Re_{\mathbf{r}}} \sum_{\varphi \in \Re_{\mathrm{r}}} \delta\left(k-m_{\varphi}\right)
$$

3. RILI detection Using Texture and Functionality Features

After segmenting the lung fields from the co-aligned 4D-CT data, the discriminative tissue features are extracted from the segmented lungs region and classified for detecting and identifying the injured regions. Two categories of features that describe lung alteration as a result of the RT are used in this chapter: (i) features in terms of Gibbs energy for the novel $7^{\text {th }}$-order contrast-offset-invariant MGRF image model and functional features, such as density ventilation, describing the air flow in the lung, and (ii) functional strain that describes elasticity of the lung tissue. Both the categories are detailed below.

a. The $7^{\text {th }}$-order textural feature: Since the RT is concentrated around the diseased part of the lung, observed texture of the affected tissue changes after the RT.

Let $\mathbb{Q}=\{0, \ldots, Q-1\}$ denote a finite set of signals (Hounsfield unit values), in the CT scan, $g: \mathbb{R} \rightarrow \mathbb{Q}$, with image signals $\mathbf{g}=(g(\mathbf{r}): \mathbf{r} \in \mathbb{R})$ Probabilistic signal 
dependencies in the images are quantified with an interaction graph, $\Upsilon=(\mathbb{R}, \mathbb{A})$, with nodes at the lattice sites (voxels), $(x, y, z) \in \mathbb{R}$, and edges, or $\operatorname{arcs}\left((x, y, z),\left(x^{\prime}, y^{\prime}, z^{\prime}\right)\right) \in$ $\mathbb{A} \subseteq \mathbb{R}^{3}$ connecting interdependent, or interacting pairs of the nodes, called neighbours. An MGRF of images is defined by a Gibbs probability distribution (GPD)

$$
\mathbf{P}=\left[P(\mathbf{g}): \mathbf{g} \in \mathbb{Q}^{|\mathbb{R}|} ; \sum_{\mathbf{g} \in \mathbb{Q}^{|\mathbb{R}|}} P(\mathbf{g})=1\right],
$$

factored over a set $\mathbb{C}$ of cliques in $\Upsilon$ supporting non-constant factors, logarithms of which are Gibbs potentials (functions of clique-wise signals) [418]. The $7^{\text {th }}$-order MGRF models the voxel's partial ordinal interaction within a radius $r$ to give an efficient description of the visual appearance of injured tissues in the CT scans.

Let a translation-invariant $K$-order interaction structure on $\mathbb{R}$ be represented by $A$, $A \geq 1$, families, $\mathbb{C}_{a} ; a=1, \ldots, A$, of $K$-order cliques, $\mathbf{c}_{a: x, y, z} \in \mathbb{C}_{a}$, of the same shape and size, for the $7^{\text {th }}$-order MGRF $K=A=7$. Every clique is associated with a certain voxel (origin), $(x, y, z) \in \mathbb{R}$, and supports the same $(K)$-variate scalar potential function, $V_{a}: \mathbb{Q}^{K} \rightarrow(-\infty, \infty)$. The GPD for this translation- and contrast/offset-invariant MGRF is specified as a general-case $K$-order exponential family distribution:

$$
P_{7}(\mathbf{g})=\frac{1}{Z} \psi(\mathbf{g}) \exp \left(-E_{7}(\mathbf{g})\right)
$$

where $\psi(\mathbf{g})$ is a core distribution, $Z$ is a normalization factor, $E_{7}(\mathbf{g})=\sum_{a=1}^{A} E_{K: a}(\mathbf{g})$ is the total Gibbs energy of all the clique families, and

$$
E_{K: a}(\mathbf{g})=\sum_{\mathbf{c}_{a: x, y, z} \in \mathbb{C}_{a}} V_{K: a}\left(g\left(x^{\prime}, y^{\prime}, z^{\prime}\right):\left(x^{\prime}, y^{\prime}, z^{\prime}\right) \in \mathbf{c}_{a: x, y, z}\right)
$$

denotes the Gibbs energy for each individual family. It describes an image texture in terms of signal dependencies (interactions) between each voxel and its neighbors depending on how the training lungs have been affected. This model accounts for partial ordinal interactions between voxel-wise signals in each particular voxel and within a radius $\rho$ from it 
for describing visual appearance of the RILI in the CT scans. The calculated Gibbs energy, $E_{K}(\mathbf{g})$, will be used to discriminate between the signals of injured tissues. While the higher energy indicates an injured tissues, the lower energy indicates a healthy tissues. To calculate $E_{K}(\mathbf{g})$, the potentials $V_{K: a}(\beta)$ are estimated for a given a training image $\mathbf{g}^{\circ}$ using the maximum likelihood estimates (MLE) for the generic $K$-order MGRF model, which generalizes the analytical approximation in [419] of the MLEs of potentials for a generic $2^{\text {nd }}$-order MGRF:

$$
v_{7: \rho}(\beta)=\frac{F_{7: \rho: \text { core }}(\beta)-F_{7: \rho}\left(\beta \mid \mathbf{g}^{\circ}\right)}{F_{7: \rho: \text { core }}(\beta)\left(1-F_{7: \rho: \text { core }}(\beta)\right)} ; \beta \in \mathbb{B}_{7}
$$

where $\beta$ denotes a numerical code of a particular contrast-offset invariant relation between seven signals; $\mathbb{B}_{7}$ is a set of these codes for all these 7 -signal co-occurrences; $F_{7: \rho}\left(\mathbf{g}^{\circ}\right)$ is an empirical marginal probability of the code $\beta ; \beta \in \mathbb{B}_{7}$, over all the 7 -voxel configurations with the radius $\rho$ in the image $\mathbf{g}^{\circ}$, and $F_{7: \rho \text { :core }}(\beta)$ is the like probability for the core distribution. The computed energy monitors changes in the tissue signals over the time and indicates the RILI development. While the higher energy suggests a severe radiation effect, the lower the Gibbs energy, the lower the injury, see Figure 43.

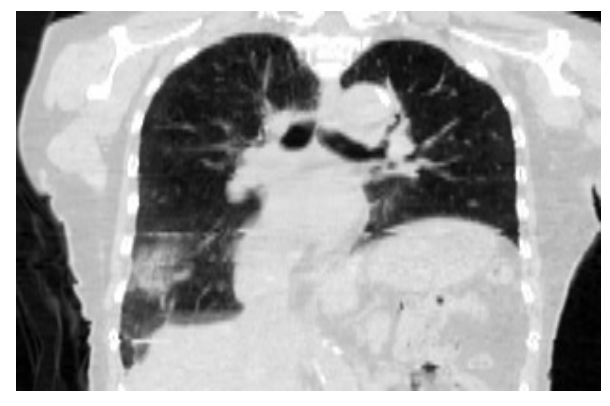

(a)

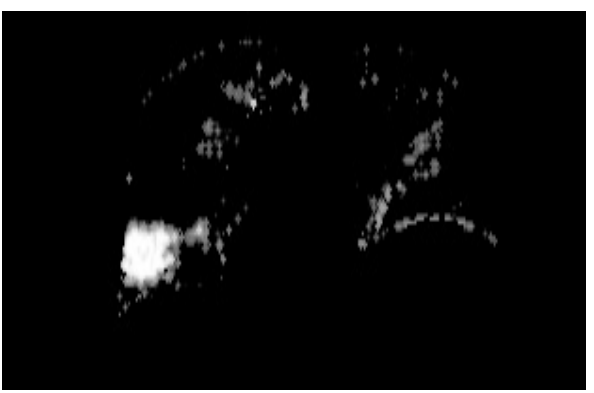

(b)

FIGURE 43: Processing steps of the proposed framework for one subject: peak inhale (a); and voxel-wise $7^{\text {th }}$-order Gibbs energies (b).

The RILI and normal regions appearances are quantified below by the voxel-wise Gibbs energies for the three $7^{\text {th }}$-order translation- and contrast/offset-invariant MGRFs, each with a single family of fixed-shape central-symmetric voxel configurations $\nu(\mathbf{r}=$ 
$(x, y, z))=\{(x, y, z) ;(x \pm \rho, y, z),(x, y \pm \rho, z),(x, y, z \pm \rho)\}$, shown in Figure 44. Their potentials and radial distances, $\rho$, between the peripheral and central voxels are learned from $\mathrm{g}^{\circ}$ by modifying the learning scenario in [420]. Algorithm 9 outlines learning the appear-

Algorithm 9 Learning the $7^{\text {th }}$-order MGRF appearance models.

1. Given a training $\mathrm{CT} \mathrm{g}^{\circ}$, find the empirical radiation injured lung tissue $(l=$ 1) and other normal tissue $(l=0)$ probability distributions, $\mathbf{F}_{l: 7: \rho}\left(\mathbf{g}^{\circ}\right)=$ $\left[F_{l: 7: \rho}\left(\beta \mid \mathbf{g}^{\circ}\right): \beta \in \mathbb{B}\right]$ of the local tirnary pattern (LTP)-based descriptors for different clique sizes $\rho \in\left\{1, \ldots, \rho_{\max }\right\}$ where the top size $\rho_{\max }=10$ in the experiments below.

2. Find the empirical distributions $\mathbf{F}_{7: \rho: \text { core }}=\left[F_{7: \rho: \operatorname{core}}(\beta): \beta \in \mathbb{B}\right]$ of the same descriptors for the core IRF $\psi(\mathrm{g})$, e.g., for an image, sampled from the core.

3. Find the approximate potentials' $\operatorname{MLE} v_{7: \rho}(\beta)=\frac{F_{7: \rho: \operatorname{core}}(\beta)-F_{7: \rho}\left(\beta \mid \mathbf{g}^{\circ}\right)}{F_{7: \rho: \operatorname{core}}(\beta)\left(1-F_{7: \rho: \operatorname{core}}(\beta)\right)} ; \beta \in \mathbb{B}_{7}$.

4. Compute partial Gibbs energies of the descriptors for equal and all other clique-wise signals over the training image for the clique sizes $\rho=1,2, \ldots, 10$ to choose the size $\rho_{l}$, making both the energies the closest one to another.

ance priors of the injured and healthy tissues. The voxel-wise energies, $E_{l: 7: \rho_{l}: x, y, z}(\mathbf{g}) ; l \in$ $\mathbb{L}$, summing the learned potentials for the seven cliques, containing the voxel $(x, y, z)$, characterize in the classification process to what extent that voxel of the image $\mathrm{g}$ can be assigned to the injured or healthy tissues in accord with their appearance priors.

b. Appearance differences in follow-up scans The exhale phase of the followup scan $I_{f}$ is co-aligned with the exhale phase of the baseline scan $I_{b}$, then subtracted to generate an intensity difference scan $I_{d}$ (see Figure 45$)$.

$$
I_{d}=I_{f}(\mathbf{x})-I_{b}(T(\mathbf{x}))
$$




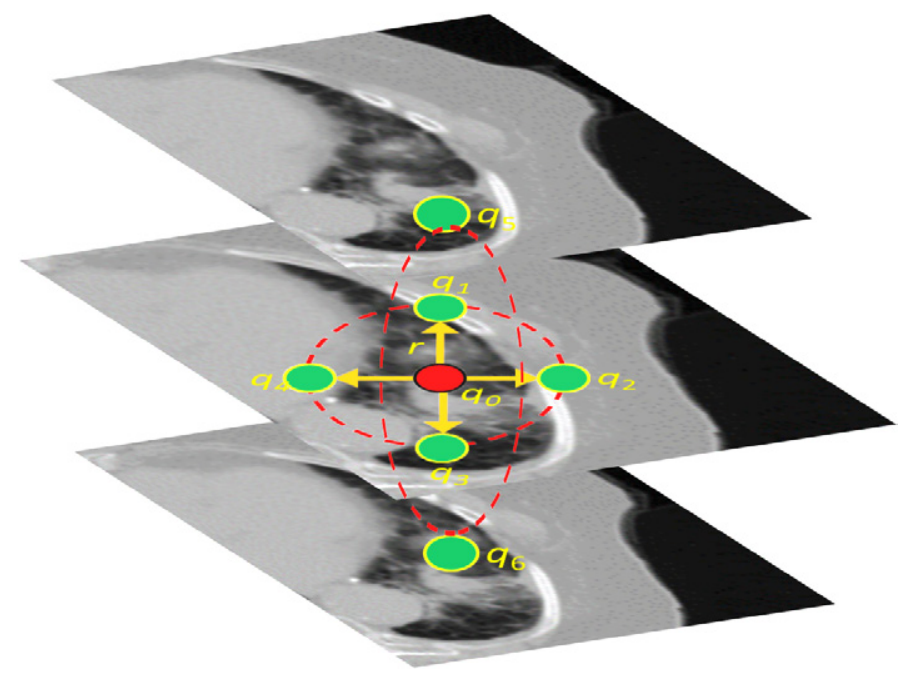

FIGURE 44: The $7^{\text {th }}$-order clique (a): signals $q_{0}, q_{1}, \ldots, q_{6}$ are at the central pixel and its six central-symmetric neighbours at the radial distance $r$, respectively.

The $I_{d}$ scan gives an initial indication of sever lung injury if there is any changes in the appearance after treatment, where positive regions indicates the progression of lung injury. The difference scan $I_{d}$ were smoothed using $3 \times 3 \times 3$ average filter to reduce the noise effects and any little changes in the inspiration capability of patients in addition to intensity changes around vessels boundaries. The difference scan gives an indication about apparent temporal changes with the lung tissues in addition to give an information about any initial injury or infection that present in the baseline scan.

c. Functionality features The second group of features, Functionality features, are extracted from the calculated voxel-wise deformation fields obtained after registering successive respiratory phases, as described in Section V.B.1.

Functional Strain is an important regional measure that describes the elasticity of the lung tissues; namely, it characterizes the deformation of lung structures. As the characteristics of the injured lung tissue change after RT, functional strain can be used for the identification of the injured lung regions. In this chapter, the Lagrangian strain has been used, which can be estimated mathematically from the gradient of the displacement vector, $\mathbf{u}(\mathbf{r})$, mapping the voxel at location $\mathbf{r}$ of the peak-exhale image to the corresponding location of the peak- 


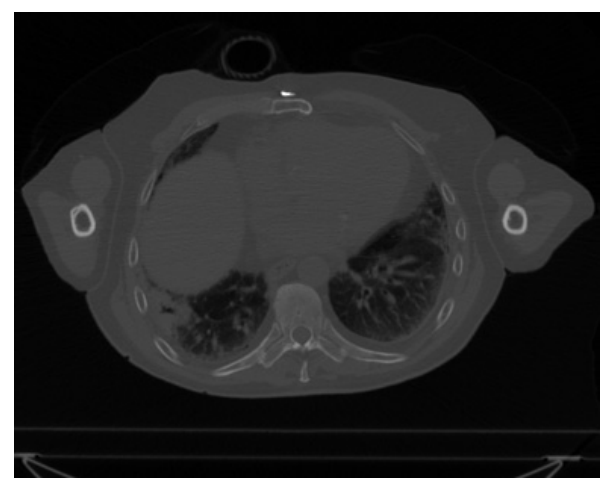

(a)

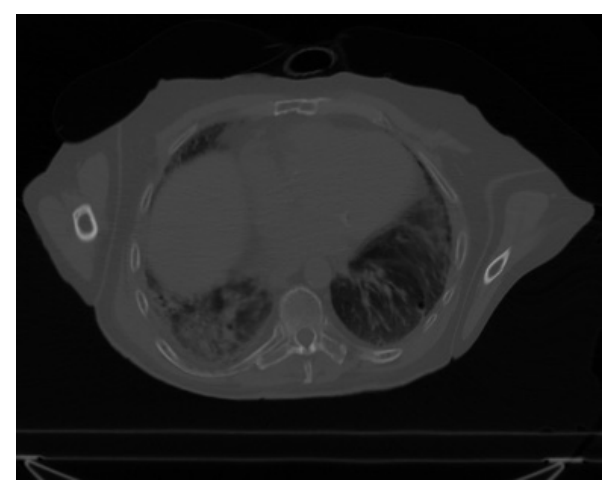

(c)

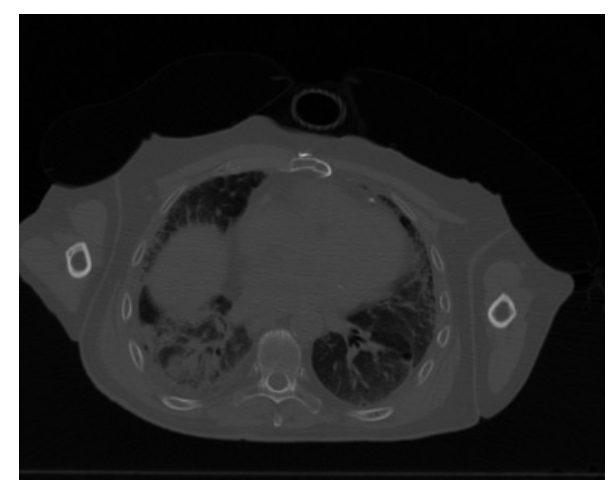

(b)

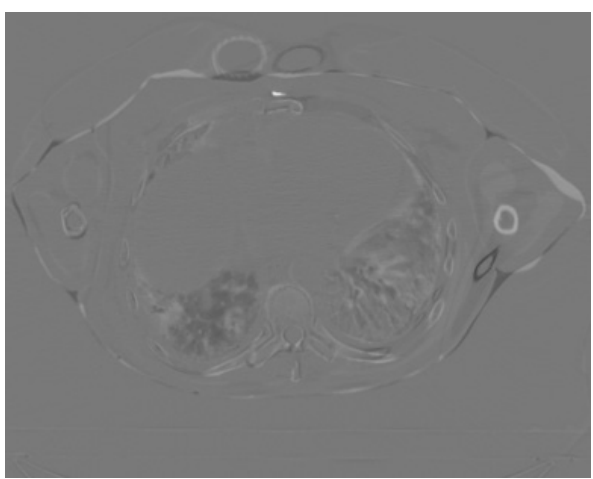

(d)

FIGURE 45: (a) baseline image $I_{b}$, (b) 3 months follow-up image $I_{f}$, (c) deformed image $I_{b}(T(x))$, and (d) the difference image $I_{d}(\mathrm{~d})$

inhale as follow:-

$$
\nabla \mathbf{u}(\mathbf{r})=\left[\begin{array}{ccc}
\frac{\partial u_{x}}{\partial x} & \frac{\partial u_{x}}{\partial y} & \frac{\partial u_{x}}{\partial z} \\
\frac{\partial u_{y}}{\partial x} & \frac{\partial u_{y}}{\partial y} & \frac{\partial u_{y}}{\partial z} \\
\frac{\partial u_{z}}{\partial x} & \frac{\partial u_{z}}{\partial y} & \frac{\partial u_{z}}{\partial z}
\end{array}\right]
$$

where the linear strain along $x, y$, and $z$ are defined as the main diagonal components, $\frac{\partial u_{x}}{\partial x}, \frac{\partial u_{y}}{\partial y}$, and $\frac{\partial u_{z}}{\partial z}$ respectively. The off-diagonal components are used to calculate the shear strain components associated with the parallel motion to the object borders as $\left(\gamma_{i j}=\right.$ $\left.\frac{\partial u_{i}}{\partial j}+\frac{\partial u_{j}}{\partial i}=\gamma_{j i}, i \neq j, i, j \in\{x, y, z\}\right)$. From the linear and shear strain notations, the 
strain can be expressed as symmetric strain tensor :-

$$
S=\left[\begin{array}{ccc}
\epsilon_{x} & \frac{\gamma_{x y}}{2} & \frac{\gamma_{x z}}{2} \\
\frac{\gamma_{y x}}{2} & \epsilon_{y} & \frac{\gamma_{y z}}{2} \\
\frac{\gamma_{z x}}{2} & \frac{\gamma_{z y}}{2} & \epsilon_{z}
\end{array}\right]=\frac{1}{2}\left[\nabla \mathbf{u}+(\nabla \mathbf{u})^{T}\right]
$$

Jacobian ventilation, measuring the partial volume change resulting from airflow during inspiration, has been documented as a good indicator to estimate regional ventilation [409]. The voxel-wise volume at the inhale phase is estimated as $V_{i n}^{\mathbf{r}}=V_{e x}^{\mathbf{r}} J_{\mathbf{r}}$ and the exhale-toinhale volume change is $\Delta V_{J}=V_{i n}^{\mathbf{r}}-V_{e x}^{\mathbf{r}}=V_{e x}^{\mathbf{r}}\left(J_{\mathbf{r}}-1\right)$, where $J_{\mathbf{r}}$ is the voxel-wise Jacobian determinant that measures the differential expansion at voxel r. Considering a small volume at the exhaled lung at point $r$ and its corresponding inhaled lung, the specific volume change is $\left(\Delta V_{J} / V_{e x}^{\mathbf{r}}\right)=\left(V_{e x}^{\mathbf{r}}\left(J_{\mathbf{r}}-1\right) / V_{e x}^{\mathbf{r}}\right)=J_{\mathbf{r}}-1$.

If $J_{\mathbf{r}}=1$, there is no expansion or contraction in the function $u$ at location $r$. If $J_{\mathbf{r}}>1$, there is a local tissue expansion; if $J_{\mathbf{r}}<1$, there is a local tissue contraction. $J_{\mathbf{r}}$ is also estimated from the gradient of the displacement fields as $J_{\mathbf{r}}=|\nabla \mathbf{u}(\mathbf{r})+I|$, where $\nabla \mathbf{u}(\mathbf{r})$ is the gradient of $\mathbf{u}(\mathbf{r})$ for each voxel in Equation (30), and $I$ is the identity matrix. Maximum shear stretch extracted from the eigendecomposition of the Jacobian matrix $J[\mathbf{x}]$ [421] of the local transformation (deformation fields).

$$
\gamma[\mathbf{x}]=\frac{\sqrt{\lambda_{N}\left(J_{\mathbf{x}}^{\top} J_{\mathbf{x}}\right)}-\sqrt{\lambda_{1}\left(J_{\mathbf{x}}^{\top} J_{\mathbf{x}}\right)}}{2}
$$

The maximum shear strain adds another functionality value for the lung tissue to be used in the discrimination process.

\section{Tissue Classification}

The introduced RILI detection framework, shown in Figure 41, is based on RF classification and incorporates regional discriminative features for better separation of data classes. RF is a machine learning technique that is increasingly being used for image 
classification and data clustering due to its powerful ability to learn the characteristics of complex data classes [422]. The main advantages of RF are its less sensitivity to data outliers, the ability to overcomes overfitting of the training set, and the ability to handle high dimensional spaces as well as large number of training examples. RF is an ensemble learning classifier that consists of many random decision trees [423] and combines two concepts: random selection of features and "bagging" [424]. The latter employs that each decision tree is typically trained with a different subset of the training samples.

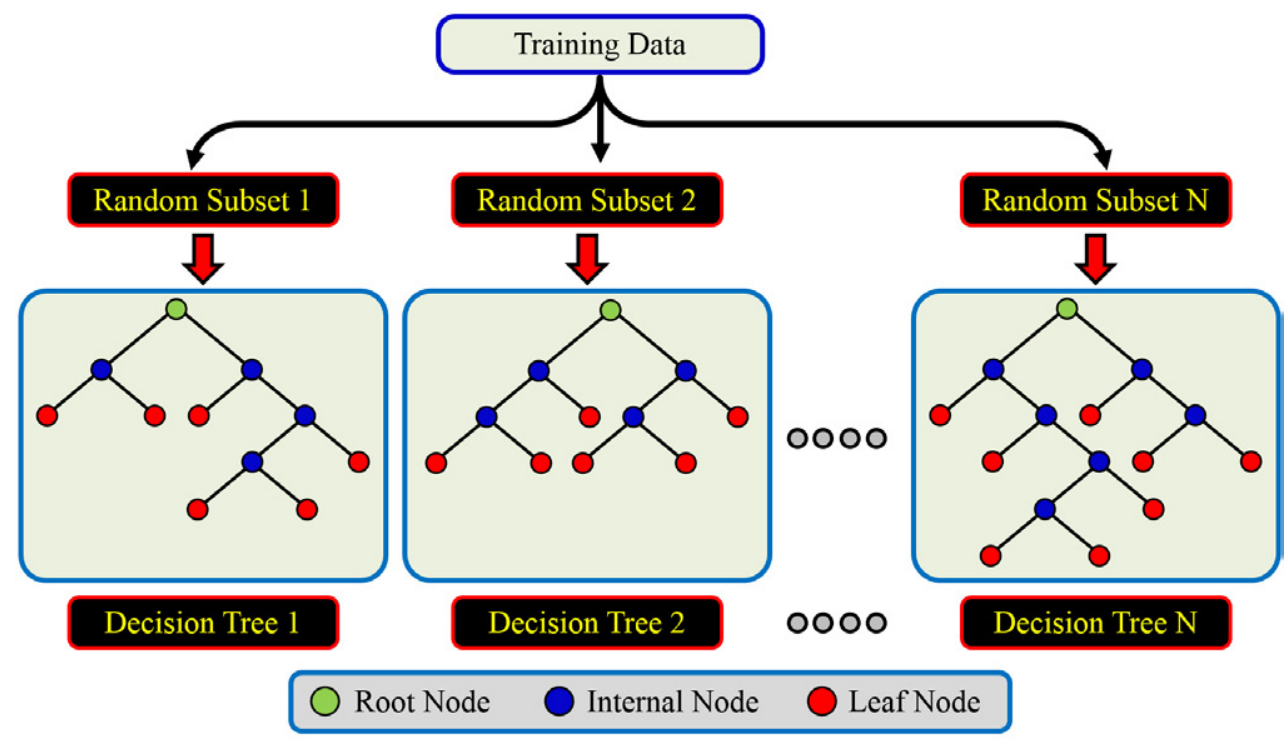

FIGURE 46: Graphical illustration of the random forest (RF) classification.

For the training phase, each decision tree uses a randomly selected subset of the training samples and features that are recursively processed along a path starting from the tree's root node using binary classification tests (see Figure 46). Those tests are performed by comparing the features' values at each internal tree node with a certain threshold, which is selected using a certain criterion. A leaf node of the tree is reached when $(i)$ the number of data samples falls below a pre-defined threshold, (ii) a predefined maximum tree depth is reached, or (iii) all data samples belong to a single class [422]. Once occurred, the label at this node is stored for the testing phase and is represented by the most frequent class of the training data at the node. In the testing phase, a new sample is processed by applying 
respective tests according to the path from the root node to the leaf it traverses. When a leaf node is reached, the tree casts a vote corresponding to the class assigned to this node in the training stage. Finally, test samples are class-labeled using majority voting over all trees' decisions and the fraction of votes for that class by all trees is used to estimate their respective class probabilities.

To classify the tissue, all the estimated features are first normalized as described in Section V.B.2 and sent to the RF classifier which learns statistical characteristics of the extracted features by using the training database containing both injured and normal lungs.

\section{Experimental Results and Conclusions}

The framework presented in this chapter uses data from a longitudinal study at the university of Louisville. Baseline scans were performed before RT treatment. The patients rescanned three, and six months after the completion of treatment course. The presented framework has been tested on clinical data sets collected for 13 patients in a study that has been approved by the University of Louisville Institutional Review Board (IRB). Patients with non-small cell lung cancer who were scheduled to receive the RT were enrolled to this study. The 4D-CT data was collected using the Philips Brilliance Big Bore CT scanner. To record the patient respiratory traces, the Varian real-time position management (RPM) system (Varian Medical Systems, Palo Alto, CA) was used. The in-plane voxel spacing ranges from $1.17 \times 1.17$ to $1.37 \times 1.37$, while the slice thickness ranges from $2 \mathrm{~mm}$ to $3 \mathrm{~mm}$.

\section{Training}

To obtain functionality and appearance features for training the proposed model, the CT data from all subjects were delineated by a radiologist. The evaluation is performed in 5-fold cross validation across the whole lungs' voxels. The voxels are split into five subsets 
where the number of injured voxels in each subset are maintained the same. For each fold, four folds are used for training, and one fold for validation.

\section{Data Augmentation}

Medical datasets usually suffer from the fact that pathological observations are significantly less frequent compared to healthy observations, As the pathological observations in medical datasets are much less frequent compared to the healthy ones, which is also holds for this study. Therefore, a serious problems may happen if simple uniform sampling is used for the learning process, as a high accuracy would be achieved if the classifier labels all of the samples as normal tissues. So, The use of imbalanced datasets for training optimization can mislead the learning technique to local optima. In this case, the predictions will be towards the dominant samples with the occupance of overfitting. To overcome this problem, the data augmentation is used to add invariance to the training data to prevent overfitting. For the training phase, as the number of injured tissues is much smaller than the healthy or unaffected tissues, The augmentation is applied only on injured tissues. Each voxel value is changed by increasing and decreasing its $\mathrm{HU}$ value by 1,2 , and 3 standard deviation std of the $3 \times 3 \times 3$ cube values centered at this voxel. Then the dataset is balanced by random upsampling the injured voxels.

\section{Scale Generation}

For each voxel neighborhood, two different size patches are extracted with size:

$3 \times 3 \times 3$, and $5 \times 5 \times 5$. This resulting in two additional scales $S c_{1}$, and $S c_{2}$. The mean value for each feature inside the two patchs scales is used as a new feature input for the RF classifier. 


\section{Results}

The classification accuracy for each fold is listed in Table 17, the accuracy for using only each of the texture features (FG1) and the functional features (FG2) in addition to both (FG3) are listed. It is clear that combining the features achieve the highest accuracy and this is because both of these features complement each other in both early and late phases.

TABLE 17: Classification Accuracy using Cross Validation for deifferent feature groups: FG1 (Texture features), FG2 (Functionality features), and FG3 (combination of all features).

\begin{tabular}{|c|c|c|c|c|c|}
\cline { 2 - 6 } \multicolumn{1}{c|}{} & \multicolumn{5}{c|}{ 5-fold Cross Validation } \\
\hline & Fold-1 & Fold-2 & Fold-3 & Fold-4 & Fold-5 \\
\hline FG1 & 90.365 & 90.801 & 90.386 & 90.761 & 90.42 \\
\hline FG2 & 91.8752 & 91.8752 & 91.6422 & 92.2196 & 92.2703 \\
\hline FG3 & 95.7147 & 95.7249 & 95.8667 & 95.8160 & 95.7046 \\
\hline
\end{tabular}

Furthermore, the classification accuracy has been evaluated by using the receiver operating characteristic (ROC), which tests the sensitivity of the proposed framework against the selected operating points (classification thresholds).

Figure 47 demonstrates the area under the ROC curve (AUC) for different feature groups. The AUC for using the FG1 only equals 0.94, while for the FG2 equals 0.96. When combining all the features in the classification process, the AUC has increased to 0.99. This enhancement highlights the advantages of integrating both the texture and functionality features as a discriminatory ones for the detection of RILI.

For regional assessment of the injured parts, The the RF classifier was applied to the voxels within a region-of-interest (ROI) determined by the radiation dose mask for all other data sets. The voxels were classified as either injured or normal tissue, and postprocessing morphological operations were used for refinement, hole filling, and removal of 


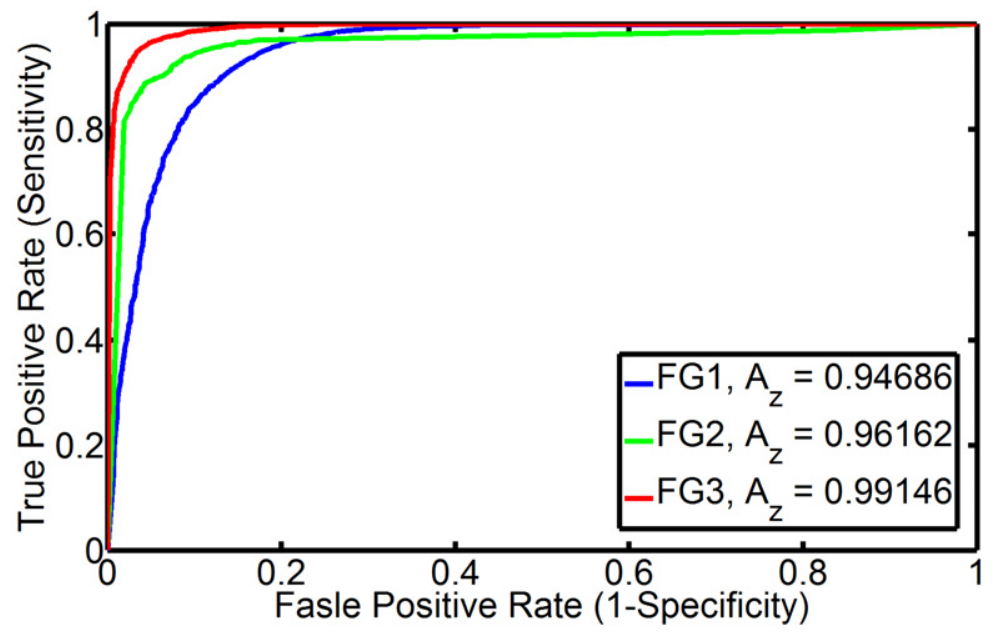

FIGURE 47: ROC curves for the RILI classification.

scattered voxels.

Figure 48 details the results of the processing steps for one subject to show that the proposed framework correctly identifies the injured lung region and demonstrates strong correlation between the estimated textural and ventilation features. Since the RILI affects the lung regions, the accuracy of the proposed framework has been evaluated with the areabased Dice similarity coefficient (DSC) [376], which characterizes agreement between the identified and true regions [376]. To obtain the ground truth in these experiments, the injured regions were delineated by a radiologist.

More identified injured regions from different subjects, projected onto different planes for visualization, are presented in Figures 49 and 50. The mean \pm standard deviation of the DSC for all the test subjects is $0.87 \pm 0.08$. 


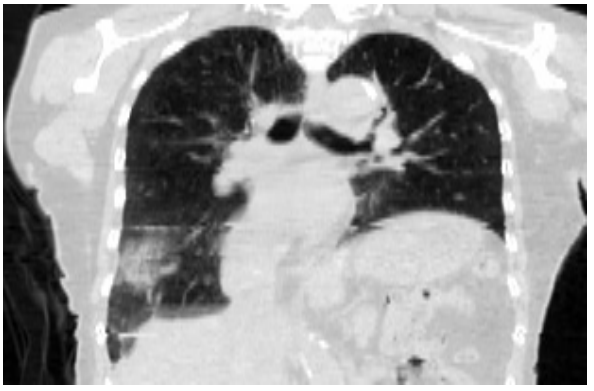

(a)

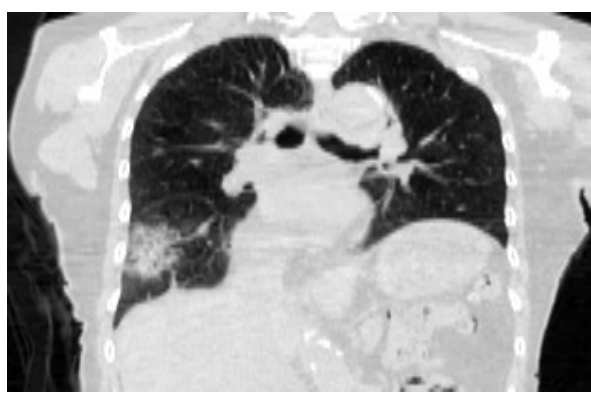

(b)

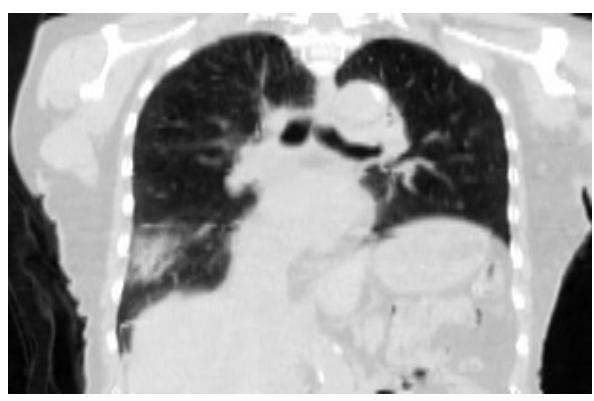

(c)

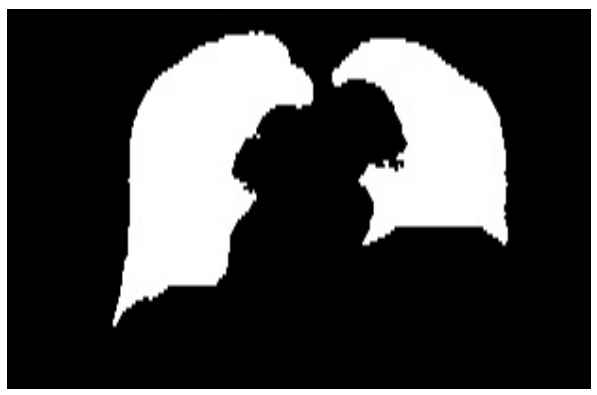

(d)

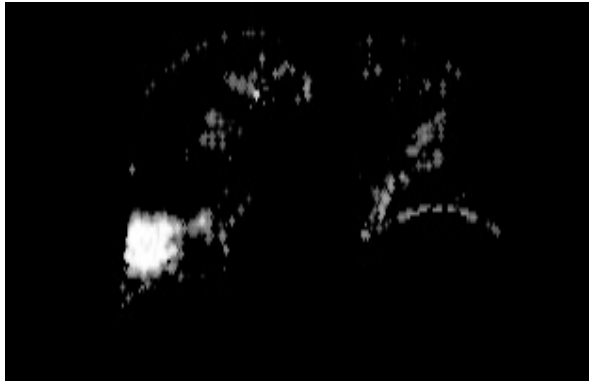

(e)

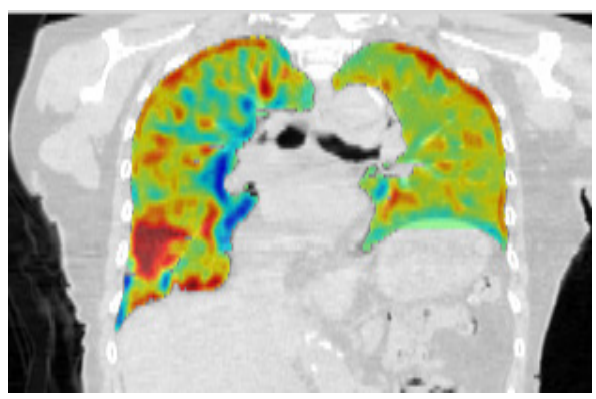

(f)

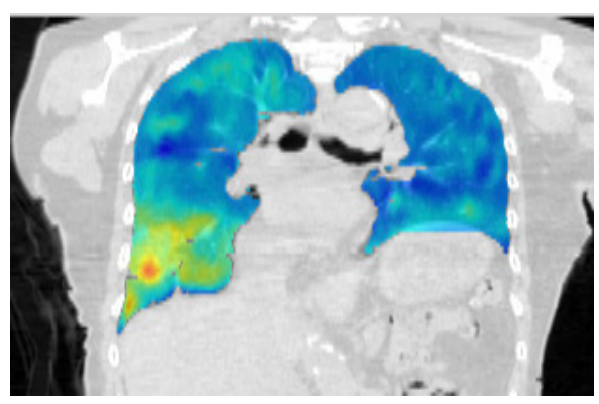

(g)

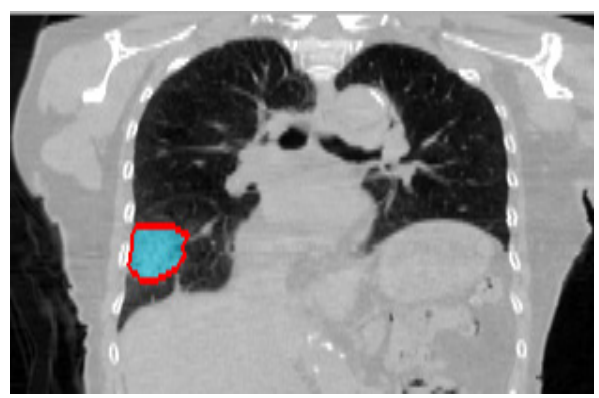

(h)

FIGURE 48: Processing steps of the proposed framework for one subject: peak inhale (a) and peak exhale (b) images; the deformed inhale image (c); lung segmentation (d); voxel-wise $7^{\text {th }}$-order Gibbs energies (e); color-coded estimated Jacobian ventilation (f) and maximal strain component (g); and identified by classification injured region (h) with respect to the ground truth (false negative errors are pink-coded). 

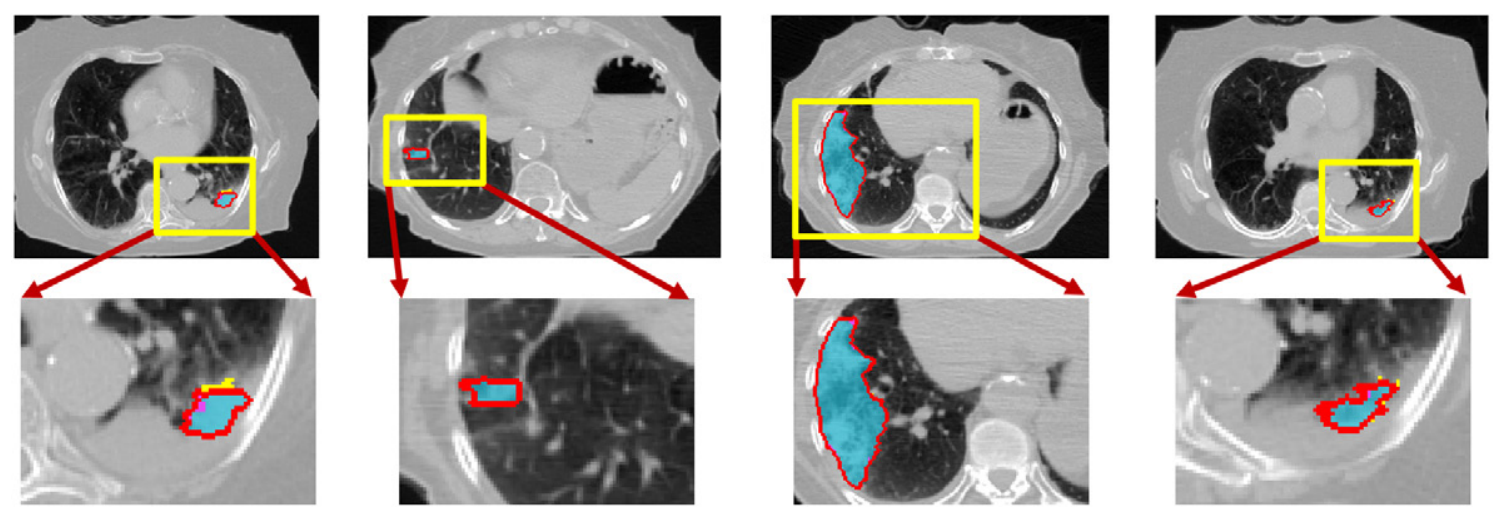

FIGURE 49: More injured lung regions from different subjects (the regions were identified with the proposed framework and projected onto the axial plane; the ground truth was obtained by a radiologist). False positive and false negative errors are yellow- and pinkcoded, respectively.

\section{Summary}

In summary, this chapter introduced a novel framework for the RILI detection from 4D-CT lung data by deformable image registration, lung fields segmentation, feature extraction, and lung tissue classification. The detection results on a cohort of 13 patients who underwent the RT confirm that the proposed framework holds the promise for early detection for lung injury. The future work plans is to test the on more data sets to more carefully assess its accuracy. In addition, more patient data will be collected with a shorter follow-up 4D-CT scans to be after the RT and before the development of RILI, which will help to assess the ability of the estimated functionality features to predict the RILI before any radiographic evidence development. The work presented in this chapter has been published in the Medical Image Computing and Computer-Assisted Intervention (MICCAI) conference [425], the International Symposium on Biomedical Imaging (ISBI) conference [426], and the International Conference on Image Processing (ICIP) [427]. 

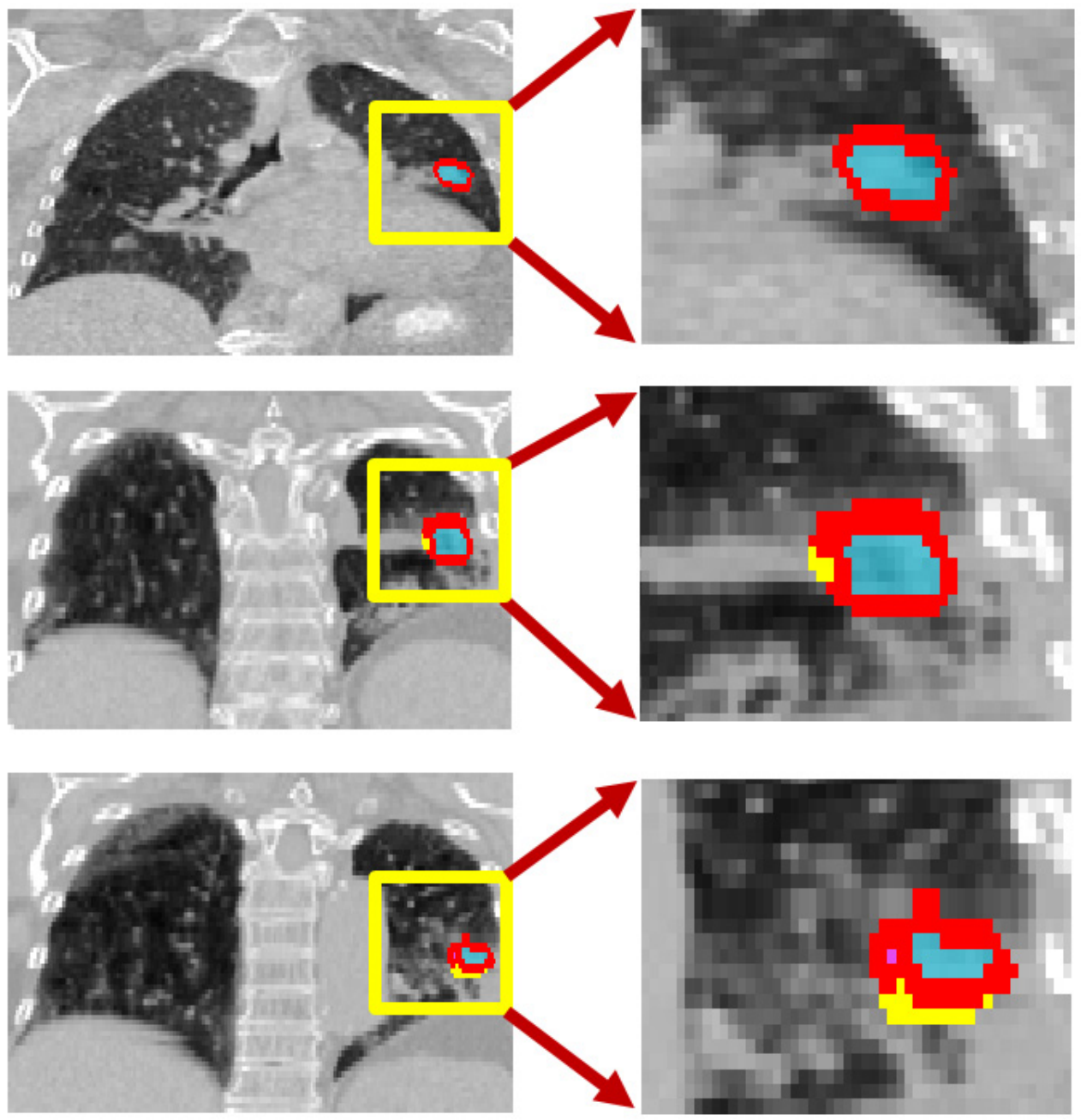

FIGURE 50: More injured lung regions from different subjects identified using the proposed framework (coronal planar projections; the ground truth by a radiologist, false positive (yellow-coded) and negative (pink-coded) errors). 


\section{CHAPTER VI CONCLUSIONS AND FUTURE WORK}

The work presented in this dissertation highlighted the ability of four dimensional computed tomography (4D-CT) chest images to provide a regional functionality evaluation for the lung. The effectiveness of 4D-CT images has been successfully tested on images collected form lung cancer patients who underwent radiation therapy (RT) treatment.

The key main advantage of the proposed computer-aided diagnostic (CAD) system for the evaluation of lung functionality is the ability of the developed models to accurately segment, handle the tissue motion, and extract discriminatory features to detect the injured tissues. These capabilities are of great importance for the medical communities to help investigate, in the case of acute pneumonitis, which local regions need attention and follow-up with appropriate treatment. Comparison results with other lung segmentation, motion handling using synthetic and in-vivo data showed the higher accuracy of the proposed models. In addition, summary of the main contributions of this dissertation are as follows:

- A novel adaptive appearance-Guided shape modeling for segmenting lungs from CT chest scans. Unlike traditional shape prior modeling, the newly developed model accounts for the database voxel locations in addition to their Hounsfield (HU) values in an adaptive way. The adaptation process is for every voxel of the chest volume, meaning that only the voxels within a predefined adapted range in adaptable search space are accountable in the shape probability calculations. This adaptive shape prior feature accurately accounts for pathological parts within the lung in addition to easy normal and healthy tissues. The shape feature is further combined in a $3 \mathrm{D}$ joint 
Markov Gibbs random field (3D MGRF) model that additionally combines the firstorder visual appearance of the lung voxels, and the second-order spatial-interaction between the neighboring lung voxels.

- A new nonrigid registration framework to accurately align temporal CT lung scans in addition to its ability to compensate for local lung deformations caused by respiratory, heart beats, and sliding motion effects is proposed. The proposed nonrigid registration deforms each voxel of the source object over a set of nested, equi-spaced surfaces (i.e., iso-surfaces) to closely match the target object, by exploiting the geometric features in addition to image intensities to avoid problems associated with nonlinear intensity variations in medical images.

- A novel $7^{\text {th }}$-order Markov Gibbs random field (MGRF) model that has the ability to accurately models the texture of healthy and injured lung tissues through simultaneously accounting for both vertical and horizontal (e.g., coronal, sagittal, and traverse) relative dependencies between voxel-wise signals. The chosen dependencies allow for variations of local 3D image contrasts and help to discriminate a particular texture of lung injury represented by a single training or query sample from other types of textures. This $7^{\text {th }}$-order MGRF model provides a balance between the speed and accuracy as the higher the order of the MGRF model, the broader its ability to learn non-homogeneous features, but the lower robustness to signal to noise and more complex learning and decision-making.

- A set of functionality features descriptors that measures the ventilation of the lung using the Jacobian of the deformations in addition to tissue elasticity using the strain components calculated from the gradient of the deformation field. 
Several possibilities for the future work of this dissertation include, but are not limited to, the following:

- Extending the presented segmentation technique to deal with segmenting different structures (multi-class labeling) of the brain [375,428-462]. This process will be of a great importance to extract and quantify the brain structures (e.g., white matter, gray matter, CSF, etc) to be used in different CAD systems for the brain.

- Extending the functionality features to be suitable to be applied for the heart [463486] to be able to diagnosis heart patients (e.g., after stem cells therapy).

- Extending the $7^{\text {th }}$-order MGRF model to be applied in the diagnosis of prostate cancer [487-500].

- Study the feasibility of applying the motion correction registration technique to the kidney $[398,501-529]$ to study kidney behavior after renal transplant.

- Testing the proposed mathematical models and learning techniques in other clinical studies, such as detection of different radiation induced injuries in different parts of the human body.

- The focus of this dissertation was on using the 4D-CT scans for the detection of lung injury as a surrogate of using nuclear imaging such as SPECT and PET. The future work will investigate the extraction of new discriminative features and the fusion of these descriptors with the clinical reports, pulmonary function tests (PFTs), and any other available imaging modality. 


\section{REFERENCES}

[1] A. Mansoor, U. Bagci, Z. Xu, B. Foster, K. N. Olivier, J. M. Elinoff, A. F. Suffredini, J. K. Udupa, and D. J. Mollura. A generic approach to pathological lung segmentation. IEEE Trans. Med. Imaging, 33(12):2293-32210, 2014.

[2] VESsel SEgmentation in the Lung 2012. http://vessel12. grand-challenge.org/, 2010.

[3] LObe and Lung Analysis 2011. http:// lola11.com/, 2011.

[4] G. T. Herman. Fundamentals of Computerized Tomography: Image Reconstruction from Projection. Springer, New York, USA, 2010.

[5] N. Hidajat, M. Wolf, A. Nunnemann, P. Liersch, B. Gebauer, U. Teichgraber, R. Schroder, and R. Felix. Survey of conventional and spiral ct doses. Radiology, 218(2):395-401, 2001.

[6] A. G. Webb. Introduction to Biomedical Imaging. Wiley-IEEE Press, 2003.

[7] B. Lee and A. Newberg. Neuroimaging in traumatic brain imaging. The J. the American Society for Experimental NeuroTherapeutics, 2(2):372-383, 2005.

[8] A. El-Baz, G. Gimel'farb, R. Falk, and M. A. El-Ghar. Automatic analysis of 3D low dose CT images for early diagnosis of lung cancer. Pattern Recognition, 42(2):10411051, 2009.

[9] B. Abdollahi, A. Soliman, A. C. Civelek, X.-F. Li, G. Gimel'farb, and A. El-Baz. A novel 3D joint MGRF framework for precise lung segmentation. In Machine Learning in Medical Imaging, pages 86-93. Springer, 2012.

[10] A. El-Baz, G. M. Beache, G. Gimel'farb, K. Suzuki, K. Okada, A. Elnakib, A. Soliman, and B. Abdollahi. Computer-aided diagnosis systems for lung cancer: Challenges and methodologies. Intern. J. Biomedical Imaging, 2013, 2013.

[11] A. El-Baz, A. Soliman, P. McClure, G. Gimel'farb, M. A. El-Ghar, and R. Falk. Early assessment of malignant lung nodules based on the spatial analysis of detected lung nodules. In Proc. IEEE Intern. Symp. on Biomedical Imaging: From Nano to Macro (ISBI'2012), pages 1463-1466, 2012.

[12] A. El-Baz, F. Khalifa, A. Elnakib, M. Nitzken, A. Soliman, P. McClure, M. A. ElGhar, and G. Gimelfarb. A novel approach for global lung registration using 3D Markov-Gibbs appearance model. In Proc. Intern. Conf. on Medical Image Computing and Computer-Assisted Intervention (MICCAI'2012), pages 114-121. Springer, 2012.

[13] P. Ou, D. S. Celermajer, G. Calcagni, F. Brunelle, D. Bonnet, and D. Sidi. Threedimensional ct scanning: a new diagnostic modality in congenital heart disease. Heart, 93(8):908-913, 2007. 
[14] S. Sheth and E. K. Fishman. Multi-detector row CT of the kidneys and urinary tract: Techniques and applications in the diagnosis of benign diseases. Radiographics, 24(2):e20, 2004.

[15] A. Jhamb, R. S. Dolas, P. K. P., and S. Mohanty. Comparative efficacy of spiral computed tomography and orthopantomography in preoperative detection of relation of inferior alveolar neurovascular bundle to the impacted mandibular third molar. $J$. Oral Maxillofac. Surg., 67(1):58-66, 2009.

[16] S. Bisdasand, G. Konstantinou, K. Surlan-Popovic, A. K. M., Baghi, T. J. Vogl, T. S. Koh, and M. G. Mack. Dynamic contrast-enhanced CT of head and neck tumors: comparison of first-pass and permeability perfusion measurements using two different commercially available tracer kinetics models. Acad. Radiol., 15(12):15801589, 2008.

[17] S. J. Swensen. Functional CT: Lung nodule evaluation. RadioGraphics, 20(1):11781181, 2000.

[18] J. G. Korporaal, C. A. T. van den Berg, C. R. L. P. N. Jeukens, G. Groenendaal, M. R. Moman, P. Luijten, M. van Vulpen, and U. A. van der Heide. Dynamic contrastenhanced CT for prostate cancer: Relationship between image noise, voxel size, and repeatability. Radiol., 256(3):976-984, 2010.

[19] K. A. Miles. Functional computed tomography in oncology. European J. Cancer, 38(16):2079-2084, 2002.

[20] P. D. Stein, A. Y. Yaekoub, F. Matta, and H. D. Sostman. Sixty-four-slice CT for diagnosis of coronary artery disease: a systematic review. Am. J. Med., 121(8):715$725,2008$.

[21] P. Verro, L. N. Tanenbaum, N. Borden, N. Eshkar, and S. Sen. Clinical application of ct angiography in acute ischemic stroke. Clin. Neurol. Neurosurg., 109(2):138-145, 2007.

[22] F. Fraioli, C. Catalano, L. Bertoletti, M. Danti, F. Fanelli, A. N. M. Cavacece, and R. Passariello. Multidetector-row CT angiography of renal artery stenosis in 50 consecutive patients: prospective interobserver comparison with DSA. Radiol. Med., 111(2):459-468, 2006.

[23] M. Dewailly, M. Rémy-Jardin, A. Duhamel, J. B. Faivre, F. Pontana, V. Deken, A. M. Bakai, and J. Remy. Computer-aided detection of acute pulmonary embolism with 64-slice multi-detector row computed tomography: Impact of the scanning conditions and overall image quality in the detection of peripheral clots. J. Comput. Assist. Tomogr., 34(1):23-30, 2010.

[24] D. W. Holdsworth and M. M. Thornton. Micro-CT in small animal and specimen imaging. Trends Biotechnol., 8(1):34-39, 2002.

[25] J. J. Vaquero, S. Redondo, E. Lage, M. Abella, A. Sisniega, G. Tapias, M. L. S. Montenegro, and M. Desco. Assessment of a new high-performance small-animal X-ray tomography. IEEE Trans. Nucl. Sci., 55(3):898-905, 2008.

[26] F. Neues and M. Epple. X-ray microcomputer tomography for the study of biomineralized endo- and exoskeletons of animals. Chem. Rev., 108(11):4734-4741, 2008. 
[27] M. E. Oest, J. C. Jones, C. Hatfield, and M. R. Prater. Micro-CT evaluation of murine fetal skeletal development yields greater morphometric precision over traditional clear-staining methods. Birth Defects Res. B Dev. Reprod. Toxicol., 83(6):582-589, 2008.

[28] K. A. Johnson. Imaging techniques for small animal imaging models of pulmonary disease: Micro-CT. Toxicolog. Pathol., 35(1):59-64, 2007.

[29] B. A. Kerr and T. V. Byzova. MicroCT: An essential tool in bone metastasis research. In L. Saba, editor, Computed Tomography-Clinical Applications, volume 1, chapter 13, pages 211-230. 2012.

[30] C. T. Badea, L. W. Hedlund, J. Cook, B. R. Berridge, and G. A. Johnson. Micro-CT imaging assessment of dobutamine-induced cardiac stress in rats. J. Pharmacolog. Toxicolog. Meth., 63(1):24-29, 2011.

[31] American cancer society: Cancer facts and figures, (2015).

[32] S. Hu, E. Hoffman, and J. Reinhardt. Automatic lung segmentation for accurate quantitation of volumetric X-ray CT images. IEEE Trans. Medical Imaging, 20(6):490-498, 2001.

[33] S. Ukil and J. M. Reinhardt. Anatomy-guided lung lobe segmentation in X-ray CT images. IEEE Trans. Medical Imaging, 28(2):202-214, 2009.

[34] E. M. van Rikxoort, B. de Hoop, S. van de Vorst, M. Prokop, and B. van Ginneken. Automatic segmentation of pulmonary segments from volumetric chest CT scans. IEEE Trans. Medical Imaging, 28(4):621-630, 2009.

[35] J. C. Ross, R. S. J. Estépar, A. Díaz, C.-F. Westin, R. Kikinis, E. K. Silverman, and G. R. Washko. Lung extraction, lobe segmentation and hierarchical region assessment for quantitative analysis on high resolution computed tomography images. In Proc. Int. Conf. Medical Imaging Computing and Computer-Assisted Intervention (MICCAI), volume 5762, pages 690-698, 2009.

[36] N. Otsu. A threshold selection method from gray-level histograms. IEEE Trans. Systems Man Cybernetics, 9(1):62-66, 1979.

[37] Y. Yim, H. Hong, and Y. G. Shin. Hybrid lung segmentation in chest CT images for computer-aided diagnosis. In Proc. of $7^{\text {th }}$ Int. Workshop on Enterprise Networking and Computing in Healthcare Industry (HEALTHCOM), pages 378-383, 2005.

[38] S. G. A. III, M. I. Giger, C. J. Moran, J. T. Blackburn, K. Doi, and H. MacMabon. Computerized detection of pulmonary nodules on CT scans. Radiographics, 19(5), 1999.

[39] S. A. III and W. Sensakovic. Automated lung segmentation for thoracic CT: Impact on computer-aided diagnosis. Acad Radiol, 11(9):1011-1021, 2004.

[40] J. Pu, J. K. Leader, B. Zheng, F. Knollmann, C. Fuhrman, F. C. Sciurba, and D. Gur. A computational geometry approach to automated pulmonary fissure segmentation in CT examinations. IEEE Trans. Medical Imaging, 28(5):710-719, 2009.

[41] J. Pu, J. Roos, C. Yi, S. Napel, G. D. R. GD, and D. S. Paik. Adaptive border marching algorithm: Automatic lung segmentation on chest CT images. Comput Med Imaging Graph, 32(6):452-462, 2008. 
[42] Q. Gao, S. Wang, D. Zhao, and J. Liu. Accurate lung segmentation for X-ray CT images. In Proc. of $3^{\text {rd }}$ Int. Conf. on Natural Computation (ICNC), volume 2, pages 275-279, 2007.

[43] Q. Wei, Y. Hu, G. Gelfand, and J. H. MacGregor. Segmentation of lung lobes in high-resolution isotropic CT image. IEEE Trans. Biomed Eng, 56(5):1383-1393, 2009.

[44] X. Ye, X. Lin, J. Dehmeshki, G. Slabaugh, and G. Beddoe. Shape-based computeraided detection of lung nodules in thoracic CT images. IEEE Trans. Biomed Eng, 56(7):1810-1820, 2009.

[45] R. C. Gonzalez and R. E. Woods. Digital Image Processing (3rd Edition). Prentice Hall, 2007.

[46] Y. Itai, K. Hyoungseop, S. Ishikawa, S. Katsuragawa, T. Ishida, K. Nakamura, and A. Yamamoto. Automatic segmentation of lung areas based on snakes and extraction of abnormal areas. In Proc. of 17th IEEE Int. Conf. on Tools with Artificial Intelligence (ICTAI), pages 377-381, 2005.

[47] M. Silveira, J. Nascimento, and J. Marques. Automatic segmentation of the lungs using robust level sets. In Proc. of $29^{\text {th }}$ IEEE Annual Int. Conf. of Medicine and Biology Society (EMBS), pages 4414-4417, 2007.

[48] P. Annangi, S. Thiruvenkadam, A. Raja, H. Xu, X. Sun, and L. Mao. A region based active contour method for X-ray lung segmentation using prior shape and low-level features. In Proc. of Int. Symposium on Biomedical Imaging (ISBI), pages 892-895, 2010.

[49] Y. Chen, H. Tagare, S. Thiruvenkadam, F. Huang, D. Wilson, K. S. Gopinath, R. W. Briggs, and E. A. Geiser. Using prior shapes in geometric active contours in a variational framework. Int. J. Computer Vision, 50(3):315-328, 2002.

[50] V. Chan and R. Vese. Active contours without edges. IEEE Trans. Image Proc, 10(2):266-277, 2001.

[51] Y. Shi, F. Qi, Z. Xue, L. Chen, K. Ito, H. Matsuo, and D. Shen. Segmenting lung fields in serial chest radiographs using both population-based and patient-specific shape statistics. IEEE Trans. Medical Imaging, 27(4):481-494, 2008.

[52] B. van Ginneken, M. B. Stegmann, and M. Loog. Segmentation of anatomical structures in chest radiographs using supervised methods: A comparative study on a public database. Med Imag Anal, 10(1):19-40, 2006.

[53] A. Tsai, W. Wells, C. Tempany, E. Grimson, and A. Willsky. Coupled multi-shape model and mutual information for medical image segmentation. In Proc. $18^{\text {th }}$ Intern. Conf. on Information Processing in Medical Imaging (IPMI 2003), pages 185-197, Ambleside, UK, July 20-25, 2003.

[54] R. C. Hardie, S. K. Rogers, T. Wilson, and A. Rogers. Performance analysis of a new computer aided detection system for identifying lung nodules on chest radiographs. Med Imag Anal, 12(3):240-258, 2008. 
[55] S. Sun, C. Bauer, and R. Beichel. Automated 3-D segmentation of lungs with lung cancer in CT data using a novel robust active shape model approach. IEEE Trans. Medical Imaging, 31(2):449-460, 2012.

[56] K. Li, X. Wu, D. Chen, and M. Sonka. Optimal surface segmentation in volumetric images-A graph-theoretic approach. IEEE Trans. Patt Anal Mach Intelli, 28(1):119134, 2006.

[57] I. Sluimer, M. Niemeijer, and B. van Ginneken. Lung field segmentation from thinslice CT scans in presence of severe pathology. In Proc. SPIE Medical Imaging, volume 5370, pages 1447-1455, 2004.

[58] I. Sluimer, M. Prokop, and B. van Ginneken. Toward automated segmentation of the pathological lung in CT. IEEE Trans. Medical Imaging, 24(8):1025-1038, 2005.

[59] M. Sofka, J. Wetzl, N. Birkbeck, J. Zhang, T. Kohlberger, J. Kaftana, J. Declerck, and S. K. Zhou. Multi-stage learning for robust lung segmentation in challenging CT volumes. In Proc. Int. Conf. Medical Imaging Computing and Computer-Assisted Intervention (MICCAI), pages 667-674, 2011.

[60] T. Kockelkorn, E. M. van Rikxoort, J. C. Grutters, and B. van Ginneken. Interactive lung segmentation in CT scans with severe abnormalities. In Proc. of Int. Symposium on Biomedical Imaging (ISBI), pages 564-567, 2010.

[61] A. Besbes and N. Paragios. Landmark-based segmentation of lungs while handling partial correspondences using sparse graph-based priors. In Proc. of Int. Symposium on Biomedical Imaging (ISBI), pages 989-995, 2011.

[62] P. Hua, Q. Song, M. Sonka, E. A. Hoffman, and J. M. Reinhardt. Segmentation of pathological and diseased lung tissue in CT images using a graph-search algorithm. In Proc. of Int. Symposium on Biomedical Imaging (ISBI), pages 2072-2075, 2011.

[63] A. El-Baz, G. gimel'farb, R. Falk, T. Holland, and T. Shaffer. A new stochatic framework for accurate lung segmentation. In Proc. Int. Conf. Medical Imaging Computing and Computer-Assisted Intervention (MICCAI), pages 322-330, 2008.

[64] A. El-Baz, G. gimel'farb, R. Falk, T. Holland, and T. Shaffer. A framework for unsupervised segmentation of lung tissues from low dose computed tomography images. In Proc. British Machine Vision Conf., pages 855-865, 2008.

[65] P. Campadelli, E. Casiraghi, and D. Artioli. A fully automated method for lung nodule detection from postero-anterior chest radiographs. IEEE Trans. Medical Imaging, 25(12):1588-1603, 2006.

[66] A. M. Mendonça, J. A. da Silva, and A. Campilho. Automatic delimitation of lung fields on chest radiographs. In Proc. of Int. Symposium on Biomedical Imaging (ISBI), volume 2, pages 1287-1290, 2004.

[67] P. Korfiatis, S. Skiadopoulos, P. Sakellaropoulos, C. Kalogeropoulou, and L. Costaridou. Combining 2D wavelet edge highlighting and 3D thresholding for lung segmentation in thin-slice CT. Br J Radiol, 80(960):996-1004, 2007.

[68] Alliance for lung cancer advocacy, support, and education: Early detection and diagnostic imaging, (2011). 
[69] L. Quekel, A. Kessels, R. Goei, and J. V. Engelshoven. Miss rate of lung cancer on the chest radiograph in clinical practice. Chest, 115(3):720-724, 1999.

[70] F. Li, S. Sone, H. Abe, H. MacMahon, S. Armato, and K. Doi. Lung cancers missed at low-dose helical CT screening in a general population: Comparison of clinical, histopathologic, and imaging findings. Radiology, 225(3):673-683, 2002.

[71] P. M. Boiselle and C. S. White. New techniques in thoracic imaging. Dekker New York, 2002.

[72] M. Kaneko, K. Eguchi, H. Ohmatsu, R. Kakinuma, T. Naruke, K. Suemasu, and N. Moriyama. Peripheral lung cancer: Screening and detection with low-dose spiral CT versus radiography. Radiology, 201(3):798-802, 1996.

[73] O. S. Miettinen and C. I. Henschke. CT screening for lung cancer: Coping with nihilistic recommendations. Radiology, 221(3):592-596, 2001.

[74] C. I. Henschke, D. P. Naidich, D. F. Yankelevitz, G. McGuinness, D. I. McCauley, et al. Early lung cancer action project: Initial finding on repeat screening. Cancer, 92(1):153-159, 2001.

[75] S. J. Swensen, J. R. Jett, T. E. Hartman, D. E. Midthun, J. A. Sloan, A. M. Sykes, G. L. Aughenbaugh, and M. A. Clemens. Lung cancer screening with CT: Mayo clinic experience. Radiology, 226(3):756-761, 2003.

[76] H. Rusinek, D. P. Naidich, G. McGuinness, B. S. Leitman, D. I. McCauley, G. A. Krinsky, K. Clayton, and H. Cohen. Pulmonary nodule detection: Low-dose versus conventional CT. Radiology, 209(1):243-249, 1998.

[77] K. Garg, R. L. Keith, T. Byers, K. Kelly, A. L. Kerzner, D. A. Lynch, and Y. E. Miller. Randomized controlled trial with low-dose spiral CT for lung cancer screening: Feasibility study and preliminary results. Radiology, 225(2):506-510, 2002.

[78] T. Nawa, T. Nakagawa, S. Kusano, Y. Kawasaki, Y. Sugawara, and H. Nakata. Lung cancer screening using low-dose spiral CT. Chest, 122(1):15-20, 2002.

[79] S. Sone, F. Li, Z. G. Yang, T. Honda, Y. Maruyama, et al. Results of three-year mass screening programme for lung cancer using mobile low-dose spiral computed tomography scanner. Br J Cancer, 84(1):25-32, 2001.

[80] S. C. B. Lo, M. T. Freedman, J. S. Lin, and S. K. Mun. Automatic lung nodule detection using profile matching and back-propagation neural network techniques. $J$ Digit Imaging, 6(1):48-54, 1993.

[81] F. Mao, W. Qian, J. Gaviria, and L. Clarke. Fragmentary window filtering for multiscale lung nodule detection. Acad Radiol, 5(4):306-311, 1998.

[82] T. Matsumoto, H. Yoshimura, K. Doi, M. Giger, A. Kano, H. MacMahon, M. Abe, and S. Montner. Image feature analysis of false-positive diagnoses produced by automated detection of lung nodules. Invest Radiol, 27(8):587-597, 1992.

[83] X. Xu, S. Katsuragawa, K. Ashizawa, H. MacMahon, and K. Doi. Analysis of image features of histograms of edge gradient for false positive reduction in lung nodule detection in chest radiographs. In Proc. SPIE Medical Imaging, volume 3338, pages 318-326, 1998. 
[84] A. A. Enquobahrie, A. P. Reeves, D. F. Yankelevitz, and C. I. Henschke. Automated detection of pulmonary nodules from whole lung helical CT scans: Performance comparison for isolated and attached nodules. In Proc. SPIE Medical Imaging, volume 5370, pages 791-800, 2004.

[85] Y. Mekada, T. Kusanagi, Y. Hayase, K. Mori, J. i. Hasegawa, J. i. Toriwaki, M. Mori, and H. Natori. Detection of small nodules from 3D chest X-ray CT images based on shape features. Proc. of Computer Assisted Radiology and Surgery (CARS), 1256:971-976, 2003.

[86] J. P. Ko and M. Betke. Chest CT: Automated nodule detection and assessment of change over timepreliminary experience. Radiology, 218(1):267-273, 2001.

[87] B. Zhao, M. S. Ginsberg, R. A. Lefkowitz, L. Jiang, C. Cooper, and L. H. Schwartz. Application of the LDM algorithm to identify small lung nodules on low-dose MSCT scans. In Proc. SPIE Medical Imaging, volume 5370, pages 818-823, 2004.

[88] S. Chang, H. Emoto, D. N. Metaxas, and L. Axe. Pulmonary micronodule detection from 3D chest CT. In Proc. Int. Conf. Medical Imaging Computing and ComputerAssisted Intervention (MICCAI), volume 3217, pages 821-828, 2004.

[89] H. Takizawa, K. Shigemoto, S. Yamamoto, T. Matsumoto, Y. Tateno, T. Iinuma, and M. Matsumoto. A recognition method of lung nodule shadows in X-Ray CT images using 3D object models. Int. J. Computer Image and Graphics, 3(4):533-545, 2003.

[90] Q. Li and K. Doi. New selective enhancement filter and its application for significant improvement of nodule detection on computed tomography. In Proc. SPIE Medical Imaging, volume 5370, pages 1-9, 2004.

[91] D. S. Paik, C. F. Beaulieu, G. D. Rubin, B. Acar, R. B. J. Jeffrey, J. Yee, J. Dey, and S. Napel. Surface normal overlap: A computer-aided detection algorithm with application to colonic polyps and lung nodules in helical CT. IEEE Trans. Medical Imaging, 23(6):661-675, 2004.

[92] P. R. S. Mendonça, R. Bhotika, S. A. Sirohey, W. D. Turner, J. V. Miller, and R. S. Avila. Model-based analysis of local shape for lesion detection in CT scans. In Proc. Int. Conf. Medical Imaging Computing and Computer-Assisted Intervention (MICCAI), volume 8, pages 688-695, 2005.

[93] Y. Lee, T. Hara, H. Fujita, S. Itoh, and T. Ishigaki. Automated detection of pulmonary nodules in helical CT images based on an improved template-matching technique. IEEE Trans. Medical Imaging, 20(7):595-604, 2001.

[94] R. Wiemker, P. Rogalla, A. Zwartkruis, and T. Blaffert. Computer aided lung nodule detection on high resolution CT data. In Proc. SPIE Medical Imaging, volume 4684, pages 677-688, 2002.

[95] W. J. Kostis, A. P. Reeves, D. F. Yankelevitz, and C. I. Henschke. Three-dimensional segmentation and growth-rate estimation of small pulmonary nodules in helical CT images. IEEE Trans. Medical Imaging, 22(10):1259-1274, 2003.

[96] K. Awai, K. Murao, A. Ozawa, M. Komi, H. Hayakawa, S. Hori, and Y. Nishimura. Pulmonary nodules at chest CT: Effect of computer-aided diagnosis on radiologists detection performance. Radiology, 230(2):347-352, 2004. 
[97] T. Ezoe, H. Takizawa, S. Yamamoto, A. Shimizu, T. Matsumoto, Y. Tateno, T. Iimura, and M. Matsumoto. An automatic detection method of lung cancers including ground glass opacities from chest X-ray CT images. In Proc. SPIE Medical Imaging, volume 4684, pages 1672-1680, 2002.

[98] C. I. Fetita, F. Prteux, C. Beigelman-Aubry, and P. Grenier. 3D automated lung nodule segmentation in HRCT. In Proc. Int. Conf. Medical Imaging Computing and Computer-Assisted Intervention (MICCAI), volume 2878, pages 626-634, 2003.

[99] M. Tanino, H. Takizawa, S. Yamamoto, T. Matsumoto, Y. Tateno, and T. Iinuma. A detection method of ground glass opacities in chest X-ray CT images using automatic clustering techniques. In Proc. SPIE Medical Imaging, volume 5032, pages 17291737, 2003.

[100] M. N. Gurcan, B. Sahiner, N. Petrick, H. P. Chan, E. A. Kazerooni, P. N. Cascade, and L. Hadjiiski. Lung nodule detection on thoracic computed tomography images: Preliminary evaluation of a computer-aided diagnosis system. Med Phys, 29(11):2552-2558, 2002.

[101] M. Kubo, K. Kubota, N. Yamada, Y. Kawata, N. Niki, et al. A CAD system for lung cancer based on low dose single-slice CT image. In Proc. SPIE Medical Imaging, volume 4684, pages 1262-1269, 2002.

[102] N. Yamada, M. Kubo, Y. Kawata, N. Niki, K. Eguchi, et al. ROI extraction of chest CT images using adaptive opening filter. In Proc. SPIE Medical Imaging, volume 5032, pages 869-876, 2003.

[103] K. Kanazawa, Y. Kawata, N. Niki, H. Satoh, H. Ohmatsu, R. Kakinuma, M. Kaneko, N. Moriyma, and K. Eguchi. Computer-aided diagnosis for pulmonary nodules based on helical CT images. Comput Med Imaging Graph, 22(2):157-167, 1998.

[104] Y. Kawata, N. Niki, H. Ohmatsu, M. Kusumoto, R. Kakinuma, K. Mori, H. Nishiyama, K. Eguchi, M. Kaneko, and N. Moriyama. Computer-aided diagnosis of pulmonary nodules using three-dimensional thoracic CT images. In Proc. Int. Conf. Medical Imaging Computing and Computer-Assisted Intervention (MICCAI), volume 2208, pages 1393-1394, 2001.

[105] M. Betke and J. P. Ko. Detection of pulmonary nodules on CT and volumetric assessment of change over time. In Proc. Int. Conf. Medical Imaging Computing and Computer-Assisted Intervention (MICCAI), pages 245-252, 1999.

[106] W. Lampeter. ANDS-V1 computer detection of lung nodules. In Proc. SPIE Medical Imaging, volume 0555, pages 253-261, 1985.

[107] T. Oda, M. Kubo, Y. Kawata, N. Niki, K. Eguchi, et al. A detection algorithm of lung cancer candidate nodules on multi-slice CT images. In Proc. SPIE Medical Imaging, volume 4684, pages 1354-1361, 2002.

[108] S. Saita, T. Oda, M. Kubo, Y. Kawata, N. Niki, et al. Nodule detection algorithm based on multi-slice CT images for lung cancer screening. In Proc. SPIE Medical Imaging, volume 5370, pages 1083-1090, 2004. 
[109] M. S. Brown, M. F. McNitt-Gray, J. G. Goldin, R. D. Suh, J. W. Sayre, and D. R. Aberle. Patient-specific models for lung nodule detection and surveillance in CT images. IEEE Trans. Medical Imaging, 20(12):1242-1250, 2001.

[110] M. L. Giger, N. Ahn, K. Doi, H. MacMahon, and C. E. Metz. Computerized detection of pulmonary nodules in digital chest images: Use of morphological filters in reducing false-positive detections. Med Phys, 17(5):861-865, 1990.

[111] J. S. Lin, P. A. Ligomenides, Y. M. F. Lure, M. T. Freedman, and S. K. Mun. Application of neural networks for improvement of lung nodule detection in radiographic images. In Symposium for Computer Assisted Radiology (S/CAR), pages 108-115, 1992.

[112] M. J. Carreira, D. Cabello, M. G. Penedo, and J. M. Pard. Computer aided lung nodule detection in chest radiography. Image Analysis Applications and Computer Graphics, 1024:331-338, 1995.

[113] R. O. Duda, P. E. Hart, and D. G. Stork. Pattern Classification (2nd Edition). NJ:Wiley Interscience, 2001.

[114] K. Fukunaga. Introduction to Statistical Pattern Recognition (2nd Edition). San Diego:Academic Press, 1990.

[115] G.-Q. Wei, L. Fan, and J. Qian. Automatic detection of nodules attached to vessels in lung CT by volume projection analysis. In Proc. Int. Conf. Medical Imaging Computing and Computer-Assisted Intervention (MICCAI), volume 2488, pages 746-752, 2002.

[116] H. Takizawa, S. Yamamoto, T. Matsumoto, Y. Tateno, T. Iinuma, and M. Matsumoto. Recognition of lung nodules from X-ray CT images using 3D Markov random field models. In Proc. SPIE Medical Imaging, volume 4684, pages 716-725, 2002.

[117] D. E. Rumelhart, G. E. Hinton, and R. J. Williams. Learning representations by back-propagating errors. Nature, 323:533-536, 1986.

[118] D. E. Rumelhart, G. E. Hinton, and R. J. Williams. Learning internal representations by error propagation. Parallel Distributed Processing, 1:318-362, 1986.

[119] L. Zhang, M. Fang, D. P. Naidich, and C. L. Novak. Consistent interactive segmentation of pulmonary ground glass nodules identified in CT studies. In Proc. SPIE Medical Imaging, volume 5370, pages 1709-1719, 2004.

[120] V. N. Vapnik. The Nature of Statistical Learning Theory. Berlin: Springer-Verlag, 1995.

[121] V. Vapnik. Statistical Learning Theory. Wiley, 1998.

[122] K. Suzuki. Pixel-based machine-learning PML in medical imaging. Int J Biomedical Imaging, 2012, 2012.

[123] K. Suzuki, I. Horiba, and N. Sugie. Neural edge enhancer for supervised edge enhancement from noisy images. IEEE Trans. Patt Anal Mach Intelli, 25(12):15821596, 2003.

[124] K. Suzuki, I. Horiba, N. Sugie, and M. Nanki. Extraction of left ventricular contours from left ventriculograms by means of a neural edge detector. IEEE Trans. Medical Imaging, 23(3):330-339, 2004. 
[125] S. B. Lo, S. A. Lou, J. S. Lin, M. T. Freedman, M. V. Chien, and S. K. Mun. Artificial convolution neural network techniques and applications for lung nodule detection. IEEE Trans. Medical Imaging, 14(4):711-718, 1995.

[126] S. C. B. Lo, H. P. Chan, J. S. Lin, H. Li, M. T. Freedman, and S. K. Mun. Artificial convolution neural network for medical image pattern recognition. Neural Networks, 8(7-8):1201-1214, 1995.

[127] J. S. Lin, S. B. Lo, A. Hasegawa, M. T. Freedman, and S. K. Mun. Reduction of false positives in lung nodule detection using a two-level neural classification. IEEE Trans. Medical Imaging, 15(2):206-217, 1996.

[128] S. C. Lo, H. Li, Y. Wang, L. Kinnard, and M. T. Freedman. A multiple circular path convolution neural network system for detection of mammographic masses. IEEE Trans. Medical Imaging, 21(2):150-158, 2002.

[129] B. Sahiner, H. P. Chan, N. Petrick, D. Wei, M. A. Helvie, D. D. Adler, and M. M. Goodsitt. Classification of mass and normal breast tissue: a convolution neural network classifier with spatial domain and texture images. IEEE Trans. Medical Imaging, 15(5):598-610, 1996.

[130] D. Wei, R. M. Nishikawa, and K. Doi. Application of texture analysis and shiftinvariant artificial neural network to microcalcification cluster detection. Radiology, 201:696-696, 1996.

[131] W. Zhang, K. Doi, M. L. Giger, R. M. Nishikawa, and R. A. Schmidt. An improved shift-invariant artificial neural network for computerized detection of clustered microcalcifications in digital mammograms. Med Phys, 23(4):595-601, 1996.

[132] W. Zhang, K. Doi, M. L. Giger, Y. Wu, R. M. Nishikawa, and R. A. Schmidt. Computerized detection of clustered microcalcifications in digital mammograms using a shift-invariant artificial neural network. Med Phys, 21(4):517-524, 1994.

[133] K. Suzuki, S. G. A. 3rd, F. Li, S. Sone, and K. Doi. Massive training artificial neural network (MTANN) for reduction of false positives in computerized detection of lung nodules in low-dose computed tomography. Med Phys, 30(7):1602-1617, 2003.

[134] K. Suzuki, H. Yoshida, J. Nappi, and A. H. Dachman. Massive-training artificial neural network (MTANN) for reduction of false positives in computer-aided detection of polyps: Suppression of rectal tubes. Med Phys, 33(10):3814-3824, 2006.

[135] K. Suzuki, H. Abe, H. MacMahon, and K. Doi. Image-processing technique for suppressing ribs in chest radiographs by means of massive training artificial neural network (MTANN). IEEE Trans. Medical Imaging, 25(4):406-416, 2006.

[136] K. Suzuki. A supervised 'lesion-enhancement' filter by use of a massive-training artificial neural network (MTANN) in computer-aided diagnosis (CAD). Physics in Medicine and Biology, 54(18):S31-S45, 2009.

[137] K. Suzuki, J. Shiraishi, H. Abe, H. MacMahon, and K. Doi. False-positive reduction in computer-aided diagnostic scheme for detecting nodules in chest radiographs by means of massive training artificial neural network. Acad Radiol, 12(2):191-201, 2005. 
[138] H. Arimura, S. Katsuragawa, K. Suzuki, F. Li, J. Shiraishi, S. Sone, and K. Doi. Computerized scheme for automated detection of lung nodules in low-dose computed tomography images for lung cancer screening. Acad Radiol, 11(6):617-629, 2004.

[139] K. Suzuki, F. Li, S. Sone, and K. Doi. Computer-aided diagnostic scheme for distinction between benign and malignant nodules in thoracic low-dose CT by use of massive training artificial neural network. IEEE Trans. Medical Imaging, 24(9):11381150, 2005.

[140] K. Suzuki, D. C. Rockey, and A. H. Dachman. CT colonography: Advanced computer-aided detection scheme utilizing mtanns for detection of missed polyps in a multicenter clinical trial. Med Phys, 37(1):12-21, 2010.

[141] K. Suzuki, H. Yoshida, J. Näppi, S. G. Armato, 3rd, and A. H. Dachman. Mixture of expert 3D massive-training anns for reduction of multiple types of false positives in cad for detection of polyps in CT colonography. Med Phys, 35(2):694-703, 2008.

[142] K. Suzuki, J. Zhang, and J. Xu. Massive-training artificial neural network coupled with laplacian-eigenfunction-based dimensionality reduction for computer-aided detection of polyps in CT colonography. IEEE Trans. Medical Imaging, 29(11):19071917, 2010.

[143] J. Xu and K. Suzuki. Massive-training support vector regression and gaussian process for false-positive reduction in computer-aided detection of polyps in CT colonography. Med Phys, 38(4):1888-1902, 2011.

[144] F. Li, H. Arimura, K. Suzuki, J. Shiraishi, Q. Li, H. Abe, R. Engelmann, S. Sone, H. MacMahon, and K. Doi. Computer-aided detection of peripheral lung cancers missed at CT: ROC analyses without and with localization. Radiology, 237(2):684690, 2005.

[145] M. L. Giger, K. T. Bae, and H. MacMahon. Computerized detection of pulmonary nodules in computed tomography images. Invest Radiol, 29(4):459-465, 1994.

[146] S. G. A. 3rd, M. L. Giger, and H. MacMahon. Automated detection of lung nodules in CT scans: Preliminary results. Med Phys, 28(8):1552-1561, 2001.

[147] M. M. Trivede and J. C. Bezdek. Low-level segmentation of aerial images with fuzzy clustering. IEEE Trans. Systems Man Cybernetics, 16(4):589-598, 1986.

[148] K. Suzuki and K. Doi. How can a massive training artificial neural network (MTANN) be trained with a small number of cases in the distinction between nodules and vessels in thoracic CT? Acad Radiol, 12(10):1333-1341, 2005.

[149] H. P. Chan, B. Sahiner, R. F. Wagner, and N. Petrick. Classifier design for computeraided diagnosis: Effects of finite sample size on the mean performance of classical and neural network classifiers. Med Phys, 26(12):2654-2668, 1999.

[150] B. Sahiner, H. P. Chan, and L. Hadjiiski. Classifier performance prediction for computer-aided diagnosis using a limited dataset. Med Phys, 35(4):1559-1570, 2008. 
[151] A. A. Farag, A. El-Baz, G. Gimelfarb, M. A. El-Ghar, and T. Eldiasty. Quantitative nodule detection in low dose chest CT scans: New template modeling and evaluation for cad system design. In Proc. Int. Conf. Medical Imaging Computing and Computer-Assisted Intervention (MICCAI), volume 8, pages 720-728, 2005.

[152] Z. Ge, B. Sahiner, H. P. Chan, L. M. Hadjiiski, P. N. Cascade, N. Bogot, E. A. Kazerooni, J. Wei, and C. Zhou. Computer-aided detection of lung nodules: False positive reduction using a 3D gradient field method and 3D ellipsoid fitting. Med Phys, 32(8):2443-2454, 2005.

[153] S. Matsumoto, H. L. Kundel, J. C. Gee, W. B. Gefter, and H. Hatabu. Pulmonary nodule detection in $\mathrm{CT}$ images with quantized convergence index filter. Med Imag Anal, 10(3):343-352, 2006.

[154] R. Yuan, P. M. Vos, and P. L. Cooperberg. Computer-aided detection in screening CT for pulmonary nodules. AJR Am J Roentgenol, 186(5):1280-1287, 2006.

[155] J. Pu, B. Zheng, J. K. Leader, X. H. Wang, and D. Gur. An automated CT based lung nodule detection scheme using geometric analysis of signed distance field. Med Phys, 35(8):3453-3461, 2008.

[156] A. Retico, P. Delogu, M. E. Fantacci, I. Gori, and A. P. Martinez. Lung nodule detection in low-dose and thin-slice computed tomography. Computers in biology and medicine, 38(4):525-534, 2008.

[157] B. Golosio, G. L. Masala, A. Piccioli, P. Oliva, M. Carpinelli, et al. A novel multithreshold method for nodule detection in lung CT. Med Phys, 36(8):3607-3618, 2009.

[158] S. G. Armato III, G. McLennan, M. F. McNitt-Gray, C. R. Meyer, D. Yankelevitz, et al. Lung image database consortium: developing a resource for the medical imaging research community. Radiology, 232(3):739-748, 2004.

[159] K. Murphy, B. van Ginneken, A. M. Schilham, B. J. de Hoop, H. A. Gietema, and M. Prokop. A large-scale evaluation of automatic pulmonary nodule detection in chest CT using local image features and k-nearest-neighbour classification. Med Imag Anal, 13(5):757-770, 2009.

[160] M. Tan, R. Deklerck, B. J. nad M. Bister, and J. Cornelis. A novel computer-aided lung nodule detection system for CT images. Med Phys, 38(10):5630-5645, 2011.

[161] T. Messay, R. C. Hardie, and S. K. Rogers. A new computationally efficient CAD system for pulmonary nodule detection in CT imagery. Med Imag Anal, 14(3):390406, 2010.

[162] A. Riccardi, T. S. Petkov, G. Ferri, M. Masotti, and R. Campanini. Computer-aided detection of lung nodules via 3D fast radial transform, scale space representation, and Zernike MIP classification. Med Phys, 38(4):1962-1971, 2011.

[163] Q. Li, F. Li, K. Suzuki, J. Shiraishi, H. Abe, R. Engelmann, Y. Nie, H. MacMahon, and K. Doi. Computer-aided diagnosis in thoracic CT. Seminars in ultrasound, CT, and MRI, 26(5):357-363, 2005.

[164] Q. Li. Recent progress in computer-aided diagnosis of lung nodules on thin-section CT. Comput Med Imaging Graph, 31(4-5):248-257, 2007. 
[165] J. G. Goldin, M. S. Brown, and I. Petkovska. Computer-aided diagnosis in lung nodule assessment. J Thoracic Imag, 23(2):97-104, 2008.

[166] I. Sluimer, A. Schilham, P. M., and B. van Ginneken. Computer analysis of computed tomography scans of the lung: A survey. IEEE Trans. Medical Imaging, 25(4):385-405, 2006.

[167] D. F. Yankelevitz, A. P. Reeves, W. J. Kostis, B. Zhao, and C. I. Henschke. Small pulmonary nodules: Volumetrically determined growth rates based on CT evaluation. Radiology, 217(1):251-256, 2000.

[168] J. P. Ko, H. Rusinek, E. L. Jacobs, J. S. Babb, M. Betke, G. McGuinness, and D. P. Naidich. Small pulmonary nodules: Volume measurement at chest CT - phantom study. Radiology, 228(3):864-870, 2003.

[169] C. C. Jaffe. Measures of response: RECIST, WHO, and new alternatives. J Clin Oncol, 24(20):3245-3251, 2006.

[170] M. A. Gavrielides, L. M. Kinnard, K. J. Myers, and N. Petrick. Noncalcified lung nodules: Volumetric assessment with thoracic CT. Radiology, 251(1):26-37, 2009.

[171] S. G. Armato III, F. Li, M. L. Giger, H. MacMahon, S. Sone, and K. Doi. Lung cancer: Performance of automated lung nodule detection applied to cancers missed in a CT screening program. Radiology, 225(3):685-692, 2002.

[172] Y. Kawata, N. Niki, H. Ohmatsu, R. Kakimuma, K. Eguchi, M. kaneko, and N. Moriyama. Classification of pulmonary nodules in thin-section CT images based on shape characterization. In Proc. IEEE Int. Conf. Image Processing (ICIP), volume 3, pages 528-530, 1997.

[173] T. Kubota and K. Okada. Estimating diameters of pulmonary nodules with competition-diffusion and robust ellipsoid fit. In Proc. ICCV Workshop on Computer Vision for Biomedical Image Applications, pages 324-334, 2005.

[174] T. Kubota, A. Jerebko, M. Dewan, M. Salganicoff, and A. Krishnan. Segmentation of pulmonary nodules of various densities with morphological approaches and convexity models. Med Imag Anal, 15(1):133-154, 2011.

[175] J. Bi, S. Periaswamy, K. Okada, T. Kubota, G. Fung, M. Salganicoff, and R. B. Rao. Computer aided detection via asymmetric cascade of sparse hyperplane classifiers. In Proc. ACM SIGKDD, pages 837-844, 2006.

[176] M. C. Lee, R. Wiemker, L. Boroczky, K. Sungur-Stasik, A. D. Cann, A. C. Borczuk, S. M. Kawut, and C. A. Powell. Impact of segmentation uncertainties on computeraided diagnosis of pulmonary nodules. Int J CARS, 3(6):551-558, 2008.

[177] C. I. Henschke, D. I. McCauley, D. F. Yankelevitz, D. P. Naidich, G. McGuinness, et al. Early Lung Cancer Action Project: Overall design and findings from baseline screening. The Lancet, 354(9173):99-105, 1999.

[178] C. I. Henschke, D. F. Yankelevitz, R. Mirtcheva, G. McGuinness, D. I. McCauley, and O. S. Miettinen. CT screening for lung cancer: Frequency and significance of part-solid and nonsolid nodules. AJR Am J Roentgenol, 178(5):1053-1057, 2002. 
[179] M. C. B. Godoy and D. P. Naidich. Subsolid pulmonary nodules and the spectrum of peripheral adenocarcinomas of the lung: Recommended interim guidelines for assessment and management. Radiology, 253(3):606-622, 2009.

[180] J. H. Min, H. Y. Lee, K. S. Lee, J. Han, K. Park, M. Ahn, and S. J. Lee. Stepwise evolution from a focal pure pulmonary ground-glass opacity nodule into an invasive lung adenocarcinoma: An observation for more than 10 years. Lung Cancer, 69(1):123-126, 2010.

[181] B. van Ginneken, B. M. ter Haar Romeny, and M. A. Viergever. Computeraided diagnosis in chest radiography: A survey. IEEE Trans. Medical Imaging, 20(12):1228-1241, 2001.

[182] E. Wei, J. Yan, M. Xu, and J. W. Zhang. A novel segmentation algorithm for pulmonary nodule in chest radiograph. In Proc. IARP Int. Conf. Pattern Recognition (ICPR), pages 1-4, 2008.

[183] B. Zhao, D. F. Yankelevitz, A. P. Reeves, and C. I. Henschke. Two-dimensional multi-criterion segmentation of pulmonary nodules on helical CT images. Med Phys, 26(6):889-895, 1999.

[184] D. F. Yankelevitz, R. Gupta, B. Zhao, and C. I. Henschke. Small pulmonary nodules: Evaluation with repeat CT - preliminary experience. Radiology, 212(2):561-566, 1999.

[185] B. Zhao, A. P. Reeves, D. F. Yankelevitz, and C. I. Henschke. Three-dimensional multicriterion automatic segmentation of pulmonary nodules of helical computed tomography images. Opt Eng, 38(08):1340-1347, 1999.

[186] R. Wiemker and A. Zwartkruis. Optimal thresholding for 3D segmentation of pulmonary nodules in high resolution CT. International Congress Series, 1230(1):653$658,2001$.

[187] W. Mullally, M. Betke, J. Wang, and J. P. Ko. Segmentation of nodules on chest computed tomography for growth assessment. Med Phys, 31(4):839-848, 2004.

[188] W. J. Kostis, D. F. Yankelevitz, A. P. Reeves, S. C. Fluture, and C. I. Henschke. Small pulmonary nodules: Reproducibility of three-dimensional volumetric measurement and estimation of time to follow-up CT. Radiology, 231(2):446-452, 2004.

[189] J. M. Kuhnigk, V. Dicken, L. Bornemann, D. Wormanns, S. Krass, and H. O. Peitgen. Fast automated segmentation and reproducible volumetry of pulmonary metastases in CT-scans for therapy monitoring. In Proc. Int. Conf. Medical Imaging Computing and Computer-Assisted Intervention (MICCAI), volume 3217, pages 933941,, 2004.

[190] J. M. Kuhnigk, V. Dicken, L. Bornemann, A. Bakai, D. Wormanns, S. Krass, and H. O. Peitgen. Morphological segmentation and partial volume analysis for volumetry of solid pulmonary lesions in thoracic CT scans. IEEE Trans. Medical Imaging, 25(4):417-434, 2006.

[191] K. Okada, V. Ramesh, A. Krishnan, M. Singh, and U. Akdemir. Robust pulmonary nodule segmentation in CT: Improving performance for juxtapleural cases. In Proc. 
Int. Conf. Medical Imaging Computing and Computer-Assisted Intervention (MICCAI), volume 8, pages 781-789, 2005.

[192] J. Dehmeshki, H. Amin, M. Valdivieso, and X. Ye. Segmentation of pulmonary nodules in thoracic CT scans: A region growing approach. IEEE Trans. Medical Imaging, 27(4):467-480, 2008.

[193] S. Diciotti, G. Picozzi, M. Falchini, M. Mascalchi, N. Villari, and G. Valli. 3-D segmentation algorithm of small lung nodules in spiral CT images. IEEE Trans. Info Tech Biomed, 12(1):7-19, 2008.

[194] T. Kubota, A. Jerebko, M. Salganicoff, M. Dewan, and A. Krishnan. Robust segmentation of pulmonary nodules of various densities: From ground-glass opacities to solid nodules. In Proc. Int. Workshop. Pulmonary Image Processing, pages 253$262,2008$.

[195] Y. Kawata, N. Niki, H. Ohmatsu, R. Kakinuma, K. Eguchi, M. Kaneko, and N. Moriyama. Quantitative surface characterization of pulmonary nodules based on thin-section CT images. IEEE Trans. Nucl Sci, 45(4):2132-2138, 1998.

[196] Y. Kawata, N. Niki, H. Ohmatsu, and N. Moriyama. A deformable surface model based on boundary and region information for pulmonary nodule segmentation from 3-D thoracic CT images. IEICE Trans Inf Syst (Inst Electron Inf Commun Eng), E86-D(9):1921-1930, 2003.

[197] A. El-Baz, A. Farag, G. Gimel'farb, R. Falk, M. A. El-Ghar, and T. Eldiasty. A framework for automatic segmentation of lung nodules from low dose chest CT scans. In Proc. IARP Int. Conf. Pattern Recognition (ICPR), volume 3, pages 611614, 2006.

[198] A. Farag, A. El-Baz, G. Gimel'farb, R. Falk, M. A. El-Ghar, and T. Eldiasty. Appearance models for robust segmentation of pulmonary nodules in 3D LDCT chest images. In Proc. Int. Conf. Medical Imaging Computing and Computer-Assisted Intervention (MICCAI), volume 9, pages 662-670, 2006.

[199] T. Waya, L. Hadjiiski, B. Sahiner, H. Chan, P. Cascade, E. Kazerooni, N. Bogot, and C. Zhou. Computer-aided diagnosis of pulmonary nodules on CT scans: Segmentation and classification using 3D active contours. Med Phys, 33(7):2323-2337, 2006.

[200] Y. Yoo, H. Shim, I. D. Yun, K. W. Lee, and S. U. Lee. Segmentation of ground glass opacities by asymmetric multi-phase deformable model. In Proc. SPIE Medical Imaging, volume 6144, pages 1278-1285, 2006.

[201] A. A. Farag, H. Abdelmunim, J. Graham, A. A. Farag, S. Elshazly, et al. Variational approach for segmentation of lung nodules. In Proc. IEEE Int. Conf. Image Processing (ICIP), pages 2157-2160, 2011.

[202] N. Xu, N. Ahuja, and R. Bansal. Automated lung nodule segmentation using dynamic programming and EM based classification. In Proc. SPIE Medical Imaging, volume 4684, pages 666-667, 2002. 
[203] J. Wang, R. Engelmann, and Q. Li. Segmentation of pulmonary nodules in three-dimensional CT images by use of a spiral-scanning technique. Med Phys, 34(12):4678-4689, 2007.

[204] Q. Wang, E. Song, R. Jin, P. Han, X. Wang, Y. Zhou, and J. Zeng. Segmentation of lung nodules in computed tomography images using dynamic programming and multidirection fusion techniques. Acad Radiol, 16(6):678-688, 2009.

[205] K. Okada, D. Comaniciu, N. Dalal, and A. Krishnan. A robust algorithm for characterizing anisotropic local structures. In Proc. Euro. Conf. Computer Vision, volume 1, pages 549-561, 2004.

[206] K. Okada, D. Comaniciu, and A. Krishnan. Scale selection for anisotropic scalespace: Application to volumetric tumor characterization. In Proc. IEEE Conf. Computer Vision and Pattern Recognition (CVPR), volume 1, pages 594-601, 2004.

[207] K. Okada, D. Comaniciu, and A. Krishnan. Robust anisotropic gaussian fitting for volumetric characterization of pulmonary nodules in multislice CT. IEEE Trans. Medical Imaging, 24(3):409-423, 2005.

[208] A. C. Jirapatnakul, S. V. Fotin, A. P. Reeves, A. M. Biancardi, D. F. Yankelevitz, and C. I. Henschke. Automated nodule location and size estimation using a multi-scale Laplacian of Gaussian filtering approach. In Proc. of $29^{\text {th }}$ IEEE Annual Int. Conf. of Medicine and Biology Society (EMBS), pages 1028-1031, 2009.

[209] S. Diciotti, S. Lombardo, G. Coppini, L. Grassi, M. Falchini, and M. Mascalchi. The LoG characteristic scale: A consistent measurement of lung nodule size in CT imaging. IEEE Trans. Medical Imaging, 29(2):397-409, 2010.

[210] L. Zhang, C. L. Zhang, T. Novak, D. P. Naidich, and D. A. Moses. A computer-based method of segmenting ground glass nodules in pulmonary CT images: Comparison to expert radiologists' interpretations. In Proc. SPIE Medical Imaging, volume 5747, pages 113-123, 2005.

[211] K. Okada, U. Akdemir, and A. Krishnan. Blob segmentation using joint spaceintensity likelihood ratio test: Application to volumetric tumor characterization. In Proc. IEEE Conf. Computer Vision and Pattern Recognition (CVPR), volume 2, pages 437-444, 2005.

[212] J. Zhou, S. Chang, D. N. Metaxas, B. Zhao, M. S. Ginsberg, and L. H. Schwartz. An automatic method for ground-glass opacity nodule detection and segmentation from CT studies. In Proc. of $29^{\text {th }}$ IEEE Annual Int. Conf. of Medicine and Biology Society (EMBS), volume 1, pages 3062-3065, 2006.

[213] J. Zhou, S. Chang, D. N. Metaxas, B. Zhao, L. H. Schwartz, and M. S. Ginsberg. Automatic detection and segmentation of ground-glass opacity nodules. In Proc. Int. Conf. Medical Imaging Computing and Computer-Assisted Intervention (MICCAI), volume 9, pages 784-791, 2006.

[214] W. A. Browder, A. P. Reeves, T. V. Apananosovich, M. D. Cham, D. F. Yankelevitz, and C. I. Henschke. Automated volumetric segmentation method for growth consistency of nonsolid pulmonary nodules in high-resolution CT. In Proc. SPIE Medical Imaging, volume 6514, page 65140Y, 2007. 
[215] Y. Tao, L. Lu, M. Dewan, A. Y. Chen, J. Corso, J. Xuan, M. Salganicoff, and A. Krishnan. Multi-level ground glass nodule detection and segmentation in CT lung images. In Proc. Int. Conf. Medical Imaging Computing and Computer-Assisted Intervention (MICCAI), volume 1, pages 715-723, 2009.

[216] B. van Ginneken. Supervised probabilistic segmentation of pulmonary nodules in CT scans. In Proc. Int. Conf. Medical Imaging Computing and Computer-Assisted Intervention (MICCAI), volume 9, pages 912-919, 2006.

[217] O. Zinoveva, D. Zinovev, S. A. Siena, D. S. Raicu, J. Furst, and S. G. Armato. A texture-based probabilistic approach for lung nodule segmentation. In Proc. Int. Conf. Image Analysis and Recognition, volume 2, pages 21-30, 2011.

[218] K. Okada, D. Comaniciu, and A. Krishnan. Robust 3D segmentation of pulmonary nodules in multislice CT images. In Proc. Int. Conf. Medical Imaging Computing and Computer-Assisted Intervention (MICCAI), volume 2, pages 881-889, 2004.

[219] S.-D. Nie, Z.-X. Chen, and L. H. Li. A CI feature-based pulmonary nodule segmentation using three-domain mean shift clustering. In Proc. Int Conf. Wavelet Analysis and Pattern Recognition, volume 223, pages 223-227, 2007.

[220] Y. J. Zheng, K. Steiner, T. Bauer, J. Y. Yu, D. G. Shen, and C. Kambhamettu. Lung nodule growth analysis from 3D CT data with a coupled segmentation and registration framework. In Proc. IEEE Workshop Mathematical Methods in Biomedical Image Analysis, pages 1-8, 2007.

[221] Y. J. Zheng, C. Kambhamettu, T. Bauer, and K. Steiner. Accurate estimation of pulmonary nodule's growth rate in CT images with nonrigid registration and precise nodule detection and segmentation. In Proc. IEEE Workshop Mathematical Methods in Biomedical Image Analysis, pages 101-108, 2009.

[222] L. R. Goodman, M. Gulsun, L. Washington, P. G. Nagy, and K. L. Piacsek. Inherent variability of CT lung nodule measurements in vivo using semiautomated volumetric measurements. AJR Am J Roentgenol, 186(4):989-994, 2006.

[223] M. Kass, A. Witkin, and D. Terzopoulos. Snakes: Active contour model. Int. J. Computer Vision, 1(4):321-331, 1988.

[224] V. Caselles, R. Kimmel, and G. Sapiro. Geodesic active contours. Int. J. Computer Vision, 22(1):61-79, 1997.

[225] V. Caselles, R. Kimmel, G. Sapiro, and C. Sbert. Minimal surfaces based on object segmentation. IEEE Trans. Patt Anal Mach Intelli, 19(4):394-398, 1997.

[226] R. Vese and V. Chan. A multiphase level set framework for image segmentation using the mumford and shah model. Int. J. Computer Vision, 50(3):271-293, 2002.

[227] A. A. Amini, T. E. Weymouth, and R. C. Jain. Using dynamic programming for solving variational problems in vision. IEEE Trans. Patt Anal Mach Intelli, 12(9):855867, 1990.

[228] D. Geiger, A. Gupta, L. A. Costa, and J. Vlontzos. Dynamic programming for detecting, tracking, and matching deformable contours. IEEE Trans. Patt Anal Mach Intelli, 17(3):249-302, 1995. 
[229] D. Comaniciu and P. Meer. Mean shift: A robust approach toward feature space analysis. IEEE Trans. Patt Anal Mach Intelli, 24(5):603-619, 2002.

[230] J. Lin. Divergence measures based on the Shannon entropy. IEEE Trans. Information Theory, 37(1):145-151, 1991.

[231] T. Lindeberg. Feature detection with automatic scale selection. Int. J. Computer Vision, 30(2):79-116, 1998.

[232] L. Breiman, J. Fiedman, C. J. Stone, and R. A. Olshen. Classification and Regression Trees. Chapman and Hall/CRC, 1984.

[233] Y. Boykov, O. Veksler, and R. Zabih. Fast approximate energy minimisation via graph cuts. IEEE Trans. Patt Anal Mach Intelli, 23(11):1222-1239, 2001.

[234] D. Rueckert, L. I. Sonoda, C. Hayes, D. L. G. Hill, M. O. Leach, and D. J. Hawkes. Non-rigid registration using free-form deformations: Application to breast MR images. IEEE Trans. Medical Imaging, 18(8):712-721, 1999.

[235] D. Wormanns, G. Kohl, E. Klotz, A. Marheine, F. Beyer, W. Heindel, and $\mathrm{S}$. Diederich. Volumetric measurements of pulmonary nodules at multi-row detector CT: In vivo reproducibility. Eur Radiol, 14(1):86-92, 2004.

[236] H. A. Gietema, C. M. Schaefer-Prokop, W. P. Mali, G. Groenewegen, and M. Prokop. Pulmonary nodules: Interscan variability of semiautomated volume measurements with multisection CT- influence of inspiration level, nodule size, and segmentation performance. Radiology, 245(3):888-894, 2007.

[237] M. F. Rinaldi, T. Bartalena, L. Braccaioli, N. Sverzellati, S. Mattioli, E. Rimondi, G. Rossi, M. Zompatori, G. Battista, and R. Canini. Three-dimensional analysis of pulmonary nodules: Variability of semiautomated volume measurements between different versions of the same software. Radiol Med, 115(3):403-412, 2010.

[238] P. A. Hein, V. C. Romano, P. Rogalla, C. Klessen, A. Lembcke, L. Bornemann, V. Dicken, B. Hamm, and H. C. Bauknecht. Variability of semiautomated lung nodule volumetry on ultralow-dose CT: Comparison with nodule volumetry on standarddose CT. J Digital Imaging, 23(1):8-17, 2010.

[239] H. Ashraf, B. de Hoop, S. B. Shaker, A. Dirksen, K. S. Bach, H. Hansen, M. Prokop, and J. H. Pedersen. Lung nodule volumetry: Segmentation algorithms within the same software package cannot be used interchangeably. Eur Radiol, 20(8):1878$1885,2010$.

[240] C. M. Park, J. M. Goo, H. J. Lee, K. G. Kim, M. J. Kang, and Y. H. Shin. Persistent pure ground-glass nodules in the lung: Interscan variability of semiautomated volume and attenuation measurements. AJR Am J Roentgenol, 195(6):408-414, 2010.

[241] B. de Hoop, H. Gietema, B. van Ginneken, P. Zanen, G. Groenewegen, and M. Prokop. A comparison of six software packages for evaluation of solid lung nodules using semi-automated volumetry: What is the minimum increase in size to detect growth in repeated CT examinations. Eur Radiol, 19(4):800-808, 2009.

[242] H. Shen, B. Goebel, and B. Odry. A new algorithm for local surface smoothing with application to chest wall nodule segmentation in lung CT data. In Proc. SPIE Medical Imaging, volume 5370, pages 1519-1526, 2004. 
[243] K. Okada, M. Singh, and V. Ramesh. Prior-constrained scale-space mean shift. In Proc. British Machine Vision Conf., pages 829-838, 2006.

[244] T. W. Way, H. P. Chan, M. M. Goodsitt, B. Sahiner, L. M. Hadjiiski, C. Zhou, and A. Chughtai. Effect of CT scanning parameters on volumetric measurements of pulmonary nodules by 3D active contour segmentation: A phantom study. Phys Med Biol, 53(5):1295-1312, 2008.

[245] H. Kobatake and S. Hashimoto. Convergence index filter for vector fields. IEEE Trans. Image Proc, 8(8):1029-1038, 1999.

[246] Y. Zheng, C. Kambhamettu, T. Bauer, and K. Steiner. Estimation of ground-glass opacity measurement in CT lung images. In Proc. Int. Conf. Medical Imaging Computing and Computer-Assisted Intervention (MICCAI), volume 11, pages 238-245, 2008.

[247] A. C. Jirapatnakul, Y. D. Mulman, A. P. Reeves, D. F. Yankelevitz, and C. I. Henschke. Segmentation of juxtapleural pulmonary nodules using a robust surface estimate. Int J Biomedical Imaging, 2011, 2011.

[248] A. P. Reeves, A. B. Chan, D. F. Yankelevitz, C. I. Henschke, B. Kressler, and W. J. Kostis. On measuring the change in size of pulmonary nodules. IEEE Trans. Medical Imaging, 25(4):435-450, 2006.

[249] S. Diciotti, S. Lombardo, M. Falchini, G. Picozzi, and M. Mascalchi. Automated segmentation refinement of small lugn nodules in CT scans by local shape analysis. IEEE Trans. Biomed Eng, 58(12):3418-3428, 2011.

[250] J. Van de Steene, N. Linthout, J. de Mey, V. Vinh-Hung, C. Claassens, M. Noppen, A. Bel, and G. Storme. Definition of gross tumor volume in lung cancer: Interobserver variability. Radiother Oncol, 62(1):37-49, 2002.

[251] A. C. Paulino and P. A. Johnstone. FDG-PET in radiotherapy treatment planning: Pandora's box? Int J Radiat Oncol Biol Phys, 59(1):4-5, 2004.

[252] K. Mah, C. B. Caldwell, Y. C. Ung, C. E. Danjoux, J. M. Balogh, S. N. Ganguli, L. E. Ehrlich, and R. Tirona. The impact of (18)FDG-PET on target and critical organs in CT-based treatment planning of patients with poorly defined non-small-cell lung carcinoma: A prospective study. Int J Radiat Oncol Biol Phys, 52(2):339-350, 2002.

[253] J. Bradley, W. L. Thorstad, S. Mutic, T. R. Miller, F. Dehdashti, B. A. Siegel, W. Bosch, and R. J. Bertrand. Impact of FDG-PET on radiation therapy volume delineation in non-small-cell lung cancer. Int J Radiat Oncol Biol Phys, 59(1):7886, 2004.

[254] P. Giraud, D. Grahek, F. Montravers, M. F. Carette, E. Deniaud-Alexandre, et al. CT and (18)F-deoxyglucose (FDG) image fusion for optimization of conformal radiotherapy of lung cancers. Int J Radiat Oncol Biol Phys, 49(5):1249-1257, 2001.

[255] Y. E. Erdi, K. Rosenzweig, A. K. Erdi, H. A. Macapinlac, Y. C. Hu, et al. Radiotherapy treatment planning for patients with non-small cell lung cancer using positron emission tomography (PET). Radiother Oncol, 62(1):51-60, 2002.

[256] U. Nestle, K. Walter, S. Schmidt, N. Licht, C. Nieder, et al. 18F-deoxyglucose positron emission tomography (FDG-PET) for the planning of radiotherapy in lung 
cancer: High impact in patients with atelectasis. Int J Radiat Oncol Biol Phys, 44(3):593-597, 1999.

[257] E. Deniaud-Alexandre, E. Touboul, D. Lerouge, D. Grahek, J. N. Foulquier, et al. Impact of computed tomography and 18F-deoxyglucose coincidence detection emission tomography image fusion for optimization of conformal radiotherapy in nonsmall-cell lung cancer. Int J Radiat Oncol Biol Phys, 63(5):1432-1441, 2005.

[258] U. Nestle, S. Kremp, A. Schaefer-Schuler, C. Sebastian-Welsch, D. Hellwig, C. Rbe, and C. M. Kirsch. Comparison of different methods for delineation of 18F-FDG PET-positive tissue for target volume definition in radiotherapy of patients with nonsmall cell lung cancer. $J$ Nucl Med, 46(8):1342-1348, 2005.

[259] Q. C. Black, I. S. Grills, L. L. Kestin, C. Y. Wong, J. W. Wong, A. A. Martinez, and D. Yan. Defining a radiotherapy target with positron emission tomography. Int $J$ Radiat Oncol Biol Phys, 60(4):1272-1282, 2004.

[260] J. B. Davis, B. Reiner, M. Huser, C. Burger, G. Székely, and I. F. Ciernik. Assessment of 18F PET signals for automatic target volume definition in radiotherapy treatment planning. Radiother Oncol, 80(1):43-50, 2006.

[261] J. F. Daisne, M. Sibomana, A. Bol, T. Doumont, M. Lonneux, and V. Grégoire. Tridimensional automatic segmentation of PET volumes based on measured sourceto-background ratios: Influence of reconstruction algorithms. Radiother Oncol, 69(3):247-250, 2003.

[262] J. A. van Dalen, A. L. Hoffmann, V. Dicken, W. V. Vogel, B. Wiering, T. J. Ruers, N. Karssemeijer, and W. J. Oyen. A novel iterative method for lesion delineation and volumetric quantification with FDG PET. Nuclear Medicine Communications, 28(6):485-493, 2007.

[263] S. A. Nehmeh, H. El-Zeftawy, C. Greco, J. Schwartz, Y. E. Erdi, A. Kirov, C. R. Schmidtlein, A. B. Gyau, S. M. Larson, and J. L. Humm. An iterative technique to segment PET lesions using a Monte Carlo based mathematical model. Med Phys, 36(10):4803-4809, 2009.

[264] C. B. Caldwell, K. Mah, M. Skinner, and C. E. Danjoux. Can PET provide the 3D extent of tumor motion for individualized internal target volumes? A phantom study of the limitations of CT and the promise of PET. Int J Radiat Oncol Biol Phys, 55(5):1381-1393, 2003.

[265] H. Ashamalla, S. Rafla, K. Parikh, B. Mokhtar, G. Goswami, S. Kambam, H. AbdelDayem, A. Guirguis, P. Ross, and A. Evola. The contribution of integrated PET/CT to the evolving definition of treatment volumes in radiation treatment planning in lung cancer. Int J Radiat Oncol Biol Phys, 63(4):1016-1023, 2005.

[266] K. J. Biehl, F. M. Kong, F. Dehdashti, J. Y. Jin, S. Mutic, I. El Naqa, B. A. Siegel, and J. D. Bradley. 18F-FDG PET definition of gross tumor volume for radiotherapy of non-small cell lung cancer: is a single standardized uptake value threshold approach appropriate? J Nucl Med, 47(11):1808-1812, 2006.

[267] D. L. Pham, C. Xu, and J. L. Prince. Current methods in medical image segmentation. Annual Review of Biomedical Engineering, 2:315-337, 2000. 
[268] M. Hatt, C. Cheze le Rest, A. Turzo, C. Roux, and D. Visvikis. A fuzzy locally adaptive bayesian segmentation approach for volume determination in PET. IEEE Trans. Medical Imaging, 28(6):881-893, 2009.

[269] M. Hatt, C. Cheze le Rest, P. Descourt, A. Dekker, D. De Ruysscher, M. Oellers, P. Lambin, O. Pradier, and V. D. Accurate automatic delineation of heterogeneous functional volumes in positron emission tomography for oncology applications. Int J Radiat Oncol Biol Phys, 77(1):301-308, 2010.

[270] J. C. Bezdek, L. O. Hall, M. C. Clark, D. B. Goldgof, and L. P. Clarke. Medical image analysis with fuzzy models. Stat Methods Med Res, 6(3):191-214, 1997.

[271] M. Hatt, F. Lamare, N. Boussion, A. Turzo, C. Collet, et al. Fuzzy hidden markov chains segmentation for volume determination and quantitation in PET. Physics in Medicine and Biology, 52(12):3467-3491, 2007.

[272] C. J. White and J. M. Brady. A semi-automatic approach to the delineation of tumour boundaries from PET data using level sets. Society of Nuclear Medicine Annual Meeting, 2005.

[273] P. Tylski, G. Bonniaud, E. Decencire, J. Stawiaski, J. Coulot, D. Lefkopoulos, and M. Ricard. 18F-FDG PET images segmentation using morphological watershed: A phantom study. In IEEE Nuclear Science Symposium Conference Record, volume 4, pages 2063-2067, 2006.

[274] W. Zhu and T. Jiang. Automation segmentation of PET image for brain tumors. IEEE Nuclear Science Symposium Conference Record, 4:2627-2629, 2003.

[275] D. W. Montgomery, A. Amira, and H. Zaidi. Fully automated segmentation of oncological PET volumes using a combined multiscale and statistical model. Med Phys, 34(2):722-36, 2007.

[276] O. Demirkaya. Lesion segmentation in whole-body images of PET. IEEE Nuclear Science Symposium Conference Record, 4:2873-2876, 2003.

[277] X. Geets, J. A. Lee, A. Bol, M. Lonneux, and V. Grégoire. A gradient-based method for segmenting FDG-PET images: Methodology and validation. Eur J Nucl Med Mol Imaging, 34(9):1427-1438, 2007.

[278] H. Li, W. L. Thorstad, K. J. Biehl, R. Laforest, Y. Su, K. I. Shoghi, E. D. Donnelly, D. A. Low, and W. Lu. A novel PET tumor delineation method based on adaptive region-growing and dual-front active contours. Med Phys, 35(8):3711-3721, 2008.

[279] H. Yu, C. Caldwell, K. Mah, and D. Mozeg. Coregistered FDG PET/CT-based textural characterization of head and neck cancer for radiation treatment planning. IEEE Trans. Medical Imaging, 28(3):374-383, 2009.

[280] I. Avazpour, R. E. Roslan, B. Bayat, M. I. Saripan, A. J. Nordin, and R. S. A. R. Abdullah. Segmenting CT images of bronchogenic carcinoma with bone metastases using PET intensity markers approach. Radiol Oncol, 43(3):180-186, 2009.

[281] S. S. Mohamed, A. M. Youssef, E. F. El-Saadany, and M. M. A. Salama. Artificial life feature selection techniques for prostrate cancer diagnosis using TRUS images. In Proc. of $2^{\text {nd }}$ Int. Conf. on Image Analysis and Recognition, pages 903-913, 2005. 
[282] B. J. Woods, B. D. Clymer, T. Kurc, J. T. Heverhagen, R. Stevens, A. Orsdemir, O. Bulan, and M. V. Knopp. Malignant-lesion segmentation using 4D co-occurrence texture analysis applied to dynamic contrast-enhanced magnetic resonance breast image data. J Magn Reson Imaging, 25(3):495-501, 2007.

[283] J. D. Kiffer, S. U. Berlangieri, A. M. Scott, G. Quong, M. Feigen, W. Schumer, C. P. Clarke, S. R. Knight, and F. J. Daniel. The contribution of 18F-fluoro-2-deoxyglucose positron emission tomographic imaging to radiotherapy planning in lung cancer. Lung Cancer, 19(3):167-177, 1998.

[284] M. T. Munley, L. B. Marks, C. Scarfone, G. S. Sibley, E. F. J. Patz, T. G. Turkington, R. J. Jaszczak, D. R. Gilland, M. S. Anscher, and R. E. Coleman. Multimodality nuclear medicine imaging in three-dimensional radiation treatment planning for lung cancer: challenges and prospect. Lung Cancer, 23(2):105-114, 1999.

[285] A. van Der Wel, S. Nijsten, M. Hochstenbag, R. Lamers, L. Boersma, et al. Increased therapeutic ratio by 18 FDG-PET CT planning in patients with clinical CT stage N2N3M0 non-small-cell lung cancer: A modeling study. Int J Radiat Oncol Biol Phys, 61(3):649-655, 2005.

[286] R. J. Steenbakkers, J. C. Duppen, I. Fitton, K. E. Deurloo, L. J. Zijp, et al. Reduction of observer variation using matched CT-PET for lung cancer delineation: A threedimensional analysis. Int J Radiat Oncol Biol Phys, 64(2):435-448, 2006.

[287] J. L. Fox, R. R., O. W., Y. E., E. Y., N. S., L. S. A., and R. K. E. Does registration of PET and planning CT images decrease interobserver and intraobserver variation in delineating tumor volumes for nonsmall-cell lung cancer? Int J Radiat Oncol Biol Phys, 62(1):70-75, 2005.

[288] D. De Ruysscher, S. Wanders, E. van Haren, M. Hochstenbag, W. Geeraedts, et al. Selective mediastinal node irradiation based on FDG-PET scan data in patients with non-small-cell lung cancer: a prospective clinical study. Int J Radiat Oncol Biol Phys, 62(4):988-994, 2005.

[289] A. P. Reeves, A. M. Biancardi, T. V. Apanasovich, C. R. Meyer, H. MacMahon, et al. The lung image database consortium (LIDC): A comparison of different size metrics for pulmonary nodule measurements. Acad Radiol, 14(12):1475-1485, 2007.

[290] J. M. Goo, T. Tongdee, R. Tongdee, K. Yeo, C. F. Hildebolt, and K. T. Bae. Volumetric measurement of synthetic lung nodules with multi-detector row CT: Effect of various image reconstruction parameters and segmentation thresholds on measurement accuracy. Radiology, 235(3):850-856, 2005.

[291] H. Bolte, T. Jahnke, F. K. Schfer, R. Wenke, B. Hoffmann, et al. Interobservervariability of lung nodule volumetry considering different segmentation algorithms and observer training levels. Eur J Radiol, 64(2):285-295, 2007.

[292] M. Das, J. Ley-Zaporozhan, H. A. Gietema, A. Czech, G. Mhlenbruch, et al. Accuracy of automated volumetry of pulmonary nodules across different multislice CT scanners. Eur Radiol, 17(8):1979-1984, 2007. 
[293] J. G. Ravenel, W. M. Leue, P. J. Nietert, J. V. Miller, K. K. Taylor, and G. A. Silvestri. Pulmonary nodule volume: Effects of reconstruction parameters on automated measurementsa phantom study. Radiology, 247(2):400-408, 2008.

[294] C. U. Vision and I. A. Group. Elcap public lung image database. http: / / www . via.cornell.edu/databases/lungdb. html, 2003.

[295] M. McNitt-Gray, S. A. Armato III, C. Meyer, A. Reeves, G. McLennan, et al. The lung image database consortium (LIDC) data collection process for nodule detection and annotation. Acad Radiol, 14(12):1464-1474, 2007.

[296] N. C. Institute. Lidc datasets. http://imaging.cancer.gov/ programsandresources/informationsystems/lidc, 2005.

[297] G. Picozzi, E. Paci, A. L. Pegna, M. Bartolucci, G. Roselli, A. D. De Francisci, S. Gabbrielli, A. Masi, N. Villari, and M. Mascalchi. Screening of lung cancer with low dose spiral CT: Results of a three year pilot study and design of the randomized clinical trial "Italung-CT". La Radiol. Med., 109:17-26, 2005.

[298] A. L. Pegna, G. Picozzi, M. Mascalchi, F. M. Carozzi, L. Carrozzi, et al. Design, recruitment and baseline results of the ITALUNG trial for lung cancer screening with low-dose CT. Lung Cancer, 64(1):34-40, 2009.

[299] C. E. Metz. ROC methodology in radiologic imaging. Invest Radiol, 21(9):720-733, 1986.

[300] J. A. Hanley and B. J. McNeil. A method of comparing the areas under receiver operating characteristic curves derived from the same cases. Radiology, 148(3):839843, 1983.

[301] D. O. Wilson, A. Ryan, C. Fuhrman, M. Schuchert, S. Shapiro, J. M. Siegfried, and J. Weissfeld. Doubling times and CT screendetected lung cancers in the Pittsburgh Lung Screening Study. Am J Respir Crit Care Med, 185(1):85-89, 2012.

[302] P. Tao, F. Griess, Y. Lvov, M. Mineyev, B. Zhao, D. Levin, and L. Kaufman. Characterization of small nodules by automatic segmentation of X-ray computed tomography images. J Comput Assist Tomogr, 28(3):372-377, 2004.

[303] M. Petrou, L. E. Quint, B. Nan, and L. H. Baker. Pulmonary nodule volumetric measurement variability as a function of CT slice thickness and nodule morphology. AJR Am J Roentgenol, 188(2):306-312, 2007.

[304] D. T. Boll, R. C. Gilkeson, T. R. Fleiter, K. A. Blackham, J. L. Duerk, and J. S. Lewin. Volumetric assessment of pulmonary nodules with ECG-gated MDCT. AJR Am J Roentgenol, 183(5):1217-1223, 2004.

[305] O. Honda, H. Sumikawa, T. Johkoh, N. Tomiyama, N. Mihara, A. Inoue, M. Tsubamoto, J. Natsag, S. Hamada, and H. Nakamura. Computer-assisted lung nodule volumetry from multi-detector row CT: Influence of image reconstruction parameters. Eur J Radiol, 62(1):106-113, 2007.

[306] S. Sone, K. Tsushima, K. Yoshida, K. Hamanaka, T. Hanaoka, and R. Kondo. Pulmonary nodules: Preliminary experience with semiautomated volumetric evaluation by CT stratum. Acad Radiol, 17(7):900-911, 2010. 
[307] S. Toshioka, K. Kanazawa, N. Niki, H. Satoh, H. Ohmatsu, K. Eguchi, and N. Moriyama. Computer aided diagnosis system for lung cancer based on helical CT images. In Proc. SPIE Medical Imaging, volume 3034, pages 975-984, 1997.

[308] M. F. McNitt-Gray, E. M. Hart, N. Wyckoff, J. W. Sayre, J. G. Goldin, and D. R. Aberle. A pattern classification approach to characterizing solitary pulmonary nodules imaged on high resolution CT: Preliminary results. Med Phys, 26(6):880-888, 1999.

[309] A. Borghesi, D. Farina, and R. Maroldi. Small pulmonary nodules: Our preliminary experience in volumetric analysis of doubling times, Terarecon Inc, CA, Clinical Case Studies, 2007. [online]. http: / / www . terarecon . com/news / casestudy_PulmonaryNodules-BorghesiEtAl.

[310] Y. Kawata, N. Niki, H. Ohmatsu, K. Eguchi, and N. Moriyama. Shape analysis of pulmonary nodules based on thin section CT images. In Proc. SPIE Medical Imaging, volume 3034, pages 964-974, 1997.

[311] Y. Kawata, N. Niki, H. Omatsu, M. Kusumoto, R. Kakinuma, K. Mori, H. Nishiyama, K. Eguchi, M. Kaneko, and N. Moriyama. Tracking interval changes of pulmonary nodules using a sequence of threedimensional thoracic images. In Proc. SPIE Medical Imaging, volume 3979, pages 86-96, 2000.

[312] Y. Kawata, N. Niki, H. Omatsu, M. Kusumoto, R. Kakinuma, K. Mori, H. Nishiyama, K. Eguchi, M. Kaneko, and N. Moriyama. Analysis of evolving processes in pulmonary nodules using a sequence of three-dimensional thoracic images. In Proc. SPIE Medical Imaging, volume 4322, pages 1890-1901, 2001.

[313] A. C. Jirapatnakul, A. P. Reeves, A. M. Biancardi, D. F. Yankelevitz, and C. I. Henschke. Semi-automated measurement of pulmonary nodule growth without explicit segmentation. In Proc. of Int. Symposium on Biomedical Imaging (ISBI), pages 855858, 2009.

[314] M. Revel, C. Lefort, A. Bissery, M. Bienvenu, L. Aycard, G. Chatellier, and G. Frija. Pulmonary nodules: Preliminary experience with three- dimensional evaluation. $R a$ diology, 231(2):459-466, 2004.

[315] H. T. Winer-Muram, S. G. Jennings, R. D. Tarver, A. M. Aisen, M. Tann, D. J. Conces, and C. A. Meyer. Volumetric growth rate of stage I lung cancer prior to treatment: Serial CT scanning. Radiology, 223(3):798-805, 2002.

[316] S. G. Jennings, H. T. Winer-Muram, M. Tann, J. Ying, and L. Dowdeswe. Distribution of stage I lung cancer growth rates determined with serial volumetric CT measurements. Radiology, 241(2):554-563, 2006.

[317] A. Marchianò, E. Calabrò, E. Civelli, G. D. Tolla, L. F. Frigerio, et al. Pulmonary nodules: Volume repeatability at multidetector CT lung cancer screening. Radiology, 251(3):919-925, 2009.

[318] H. Jiang and K. Kelly. Theoretical prediction of lung nodule measurement accuracy under different acquisition and reconstruction conditions. In Proc. SPIE Medical Imaging, volume 5369, pages 406-412, 2004. 
[319] K. Furuya, S. Murayama, H. Soeda, J. Murakami, Y. Ichinose, H. Yabuuchi, Y. Katsuda, M. Koga, and K. Masuda. New classification of small pulmonary nodules by margin characteristics on high-resolution CT. Acta Radiologica, 40(5):496-504, 1999.

[320] Y. Kawata, N. Niki, H. Ohmatsu, R. Kakinuma, K. Mori, K. Eguchi, M. Kaneko, and N. Moriyama. Curvature based analysis of pulmonary nodules using thin-section CT images. In Proc. IARP Int. Conf. Pattern Recognition (ICPR), volume 1, pages 361363, 1998.

[321] Y. Kawata, N. Niki, H. Ohmatsu, M. Kusumoto, R. Kakinuma, K. Mori, H. Nishiyama, K. Eguchi, M. Kaneko, and N. Moriyama. Searching similar images for classification of pulmonary nodules in three-dimensional CT images. In Proc. of Int. Symposium on Biomedical Imaging (ISBI), pages 189-193, 2002.

[322] S. Kido, K. Kuriyama, M. Higashiyama, T. Kasugai, and C. Kuroda. Fractal analysis of small peripheral pulmonary nodules in thin-section CT: Evaluation of the lungnodule interfaces. J. Comput. Assist. Tomogr., 26(4):573-578, 2002.

[323] Y. Kawata, N. Niki, H. Ohmatsu, M. Kusumoto, R. Kakinuma, K. Mori, H. Nishiyama, K. Eguchi, M. Kaneko, and N. Moriyama. Computerized analysis of 3-D pulmonary nodule images in surrounding and internal structure feature spaces. In Proc. IEEE Int. Conf. Image Processing (ICIP), volume 2, pages 889-892, 2001.

[324] K. Mori, N. Niki, T. Kondo, Y. Kamiyama, T. Kodama, Y. Kawada, and N. Moriyama. Development of a novel computer-aided diagnosis system for automatic discrimination of malignant from benign solitary pulmonary nodules on thinsection dynamic computed tomography. J. Comput. Assist. Tomogr., 29(2):215-222, 2005.

[325] S. K. Shah, M. F. McNitt-Gray, S. R. Rogers, J. G. Goldin, R. D. Suh, J. W. Sayre, I. Petkovska, H. J. Kim, and D. R. Aberle. Computeraided diagnosis of the solitary pulmonary nodule. Acad Radiol, 12(5):570-575, 2005.

[326] S. K. Shah, M. F. McNitt-Gray, S. R. Rogers, J. G. Goldin, R. D. Suh, J. W. Sayre, I. Petkovska, H. J. Kim, and D. R. Aberle. Computer aided characterization of the solitary pulmonary nodule using volumetric and contrast enhancement features. Acad Radiol, 12(10):1310-1319, 2005.

[327] T. W. Way, B. Sahiner, H. P. Chan, L. Hadjiiski, P. N. Cascade, A. Chughtai, N. Bogot, and E. Kazerooni. Computer-aided diagnosis of pulmonary nodules on CT scans: Improvement of classification performance with nodule surface features. Med Phys, 36(7):3086-3098, 2009.

[328] M. Aoyama, Q. Li, S. Katsuragawa, F. Li, S. Sone, and K. Doi. Computerized scheme for determination of the likelihood measure of malignancy for pulmonary nodules on low-dose CT images. Med Phys, 30(3):387-394, 2003.

[329] W. Gurney and S. Swensen. Solitary pulmonary nodules: Determining the likelihood of malignancy with neural network analysis. Radiology, 196(3):823-829, 1995.

[330] Y. Matsuki, K. Nakamura, H. Watanabe, T. Aoki, H. Nakata, S. Katsuragawa, and K. Doi. Usefulness of an artificial neural network for differentiating benign from 
malignant pulmonary nodules on high-resolution CT: Evaluation with receiver operating characteristic analysis. AJR Am J Roentgenol, 178(3):657-663, 2002.

[331] C. Henschke, D. Yankelevitz, I. Mateescu, D. Brettle, T. Rainey, and F.Weingard. Neural networks for the analysis of small pulmonary nodules. Clincal Imaging, 21(6):390-399, 1997.

[332] S. Lo, L. Hsu, M. Freedman, Y. Lure, and H. Zhao. Classification of lung nodules in diagnostic CT: An approach based on 3D vascular features, nodule density distribution, and shape features. In Proc. SPIE Medical Imaging, volume 5032, pages 183-189, 2003.

[333] H. Chen, Y. Xu, Y. Ma, and B. Ma. Neural network ensemble-based computer-aided diagnosis for differentiation of lung nodules on CT images: Clinical evaluation. Acad Radiol, 17(5):595-602, 2010.

[334] K. Nakamura, H. Yoshida, R. Engelmann, H. MacMahon, S. Katsuragawa, T. Ishida, K. Ashizawa, and K. Doi. Computerized analysis of the likelihood of malignancy in solitary pulmonary nodules with use of artificial neural networks. Radiology, 214(3):823-830, 2000.

[335] S. Iwano, T. Nakamura, Y. Kamioka, and T. Ishigaki. Computer-aided diagnosis: a shape classification of pulmonary nodules imaged by high-resolution CT. Comput Med Imaging Graph, 29(7):565-570, 2005.

[336] S. Iwano, T. Nakamura, Y. Kamioka, M. Ikeda, and T. Ishigaki. Computer-aided differentiation of malignant from benign solitary pulmonary nodules imaged by highresolution CT. Comput Med Imaging Graph, 32(5):416-422, 2008.

[337] S. Matsuoka, Y. Kurihara, K. Yagihashi, H. Niimi, and Y. Nakajima. Peripheral solitary pulmonary nodule: CT findings in patients with pulmonary emphysema. Radiology, 235(1):266-273, 2005.

[338] M. C. Lee, L. Boroczky, K. Sungur-Stasik, A. D. Cann, A. C. Borczuk, S. M. Kawut, and C. A. Powell. Computer-aided diagnosis of pulmonary nodules using a twostep approach for feature selection and classifier ensemble construction. Artificial Intelligence in Medicine, 50(1):43-53, 2010.

[339] V. J. Lowe, J. W. Fletcher, L. Gobar, M. Lawson, P. Kirchner, et al. Prospective investigation of positron emission tomography in lung nodules. J Clin Oncol, 16(3):10751084, 1998.

[340] N. C. Gupta, J. Maloof, and E. Gunel. Probability of malignancy in solitary pulmonary nodules using fluorine-18-FDG and PET. J Nucl Med, 37(6):943-948, 1996.

[341] J. Lee, J. M. Aronchick, and A. Alavi. Accuracy of F-18 fluorodeoxyglucose positron emission tomography for the evaluation of malignancy in patients presenting with new lung abnormalities: A retrospective review. Chest, 120(6):1791-1797, 2001.

[342] N. A. Dewan, N. C. Gupta, L. S. Redepenning, J. J. Phalen, and M. P. Frick. Diagnostic efficacy of PET-FDG imaging in solitary pulmonary nodules. Potential role in evaluation and management. Chest, 104(4):997-1002, 1993. 
[343] G. J. Herder, R. P. Golding, O. S. Hoekstra, E. F. Comans, G. J. Teule, P. E. Postmus, and E. F. Smit. The performance of (18)F-fluorodeoxyglucose positron emission tomography in small solitary pulmonary nodules. Eur J Nucl Med Mol Imaging, 31(9):1231-1236, 2004.

[344] A. Halley, A. Hugentobler, P. Icard, E. Porret, F. Sobrio, J. P. Lerochais, G. Bouvard, G. Zalcman, and D. Agostini. Efficiency of 18F-FDG and 99mTc-depreotide SPECT in the diagnosis of malignancy of solitary pulmonary nodules. Eur J Nucl Med Mol Imaging, 32(9):1026-1032, 2005.

[345] J. J. Erasmus, H. P. McAdams, and J. E. Connolly. Solitary pulmonary nodules: Part II. Evaluation of the indeterminate nodule. Radiographics, 20(1):59-66, 2000.

[346] J. M. Goo, J. G. Im, K. H. Do, J. S. Yeo, J. B. Seo, H. Y. Kim, and J. K. Chung. Pulmonary tuberculoma evaluated by means of FDG PET: Findings in 10 cases. Radiology, 216(1):117-121, 2000.

[347] D. Lardinois, W. Weder, T. F. Hany, E. M. Kamel, S. Korom, B. Seifert, G. K. von Schulthess, and H. C. Steinert. Staging of non-small-cell lung cancer with integrated positron-emission tomography and computed tomography. N Engl J Med, 348(25):2500-2507, 2003.

[348] J. J. Erasmus, H. P. McAdams, E. F. J. Patz, R. E. Coleman, V. Ahuja, and P. C. Goodman. Evaluation of primary pulmonary carcinoid tumors using FDG PET. AJR Am J Roentgenol, 170(5):1369-1373, 1998.

[349] K. Higashi, Y. Ueda, H. Seki, K. Yuasa, M. Oguchi, T. Noguchi, M. Taniguchi, H. Tonami, T. Okimura, and Y. I. Fluorine-18-FDG PET imaging is negative in bronchioloalveolar lung carcinoma. J Nucl Med, 39(6):1016-1020, 1998.

[350] J. T. Annema, O. S. Hoekstra, E. F. Smit, M. Veseli, M. I. Versteegh, and K. F. Rabe. Towards a minimally invasive staging strategy in NSCLC: Analysis of PET positive mediastinal lesions by EUS-FNA. Lung Cancer, 44(1):53-60, 2004.

[351] Y. Nie, Q. Li, F. Li, Y. Pu, D. Appelbaum, and K. Doi. Integrating PET and CT information to improve diagnostic accuracy for lung nodules: A semiautomatic computeraided method. J Nucl Med, 47(7):1075-1080, 2006.

[352] Y. Nakamoto, M. Senda, T. Okada, S. Sakamoto, T. Saga, T. Higashi, and K. Togashi. Software-based fusion of PET and CT images for suspected recurrent lung cancer. Mol Imaging Biol, 10(3):147-153, 2008.

[353] Z. Keidar, N. Haim, L. Guralnik, M. Wollner, R. Bar-Shalom, A. Ben-Nun, and O. Israel. PET/CT Using 18F-FDG in suspected lung cancer recurrence: Diagnostic value and impact on patient management. J Nucl Med, 45(10):1640-1646, 2004.

[354] C. A. Yi, K. S. Lee, B. T. Kim, J. Y. Choi, O. J. Kwon, H. Kim, and M. J. Shim, Y. M. Chung. Tissue characterization of solitary pulmonary nodule: Comparative study between helical dynamic CT and integrated PET/CT. J Nucl Med, 47(3):443-450, 2006.

[355] A. Cunliffe, S. G. Armato, R. Castillo, N. Pham, T. Guerrero, and H. A. AlHallaq. Lung texture in serial thoracic computed tomography scans: Correlation 
of radiomics-based features with radiation therapy dose and radiation pneumonitis development. Int. J. Rad. Oncol.* Biol.* Phys., 91(5):1048-1056, 2015.

[356] S. G. Armato and W. F. Sensakovic. Automated lung segmentation for thoracic ct: Impact on computer-aided diagnosis1. Aca. Radiol., 11(9):1011-1021, 2004.

[357] J. Wang, F. Li, and Q. Li. Automated segmentation of lungs with severe interstitial lung disease in CT. Med. Phys., 36(10):4592-4599, 2009.

[358] T. T. Kockelkorn, C. M. Schaefer-Prokop, G. Bozovic, A. Muñoz-Barrutia, E. M. van Rikxoort, M. S. Brown, P. A. de Jong, M. A. Viergever, and B. van Ginneken. Interactive lung segmentation in abnormal human and animal chest CT scans. Medical Physics, 41(8):081915, 2014.

[359] J. Zhou, B. Zhang, G. Lasio, K. Prado, and W. D’Souza. SU-F-BRF-02: Automated lung segmentation method using atlas-based sparse shape composition with a shape constrained deformable model. Med. Phys., 41(6):399-399, 2014.

[360] I. Sluimer, M. Prokop, and B. Van Ginneken. Toward automated segmentation of the pathological lung in CT. IEEE Transactions on Medical Imaging, 24(8):1025-1038, 2005.

[361] E. M. van Rikxoort, B. de Hoop, M. A. Viergever, M. Prokop, and B. van Ginneken. Automatic lung segmentation from thoracic computed tomography scans using a hybrid approach with error detection. Med. Phys., 36:2934-2947, 2009.

[362] K. Nakagomi, A. Shimizu, H. Kobatake, M. Yakami, K. Fujimoto, and K. Togashi. Multi-shape graph cuts with neighbor prior constraints and its application to lung segmentation from a chest CT volume. Med. Image. Anal., 17:62-77, 2013.

[363] P. Korfiatis, C. Kalogeropoulou, A. Karahaliou, A. Kazantzi, S. Skiadopoulos, and L. Costaridou. Texture classification-based segmentation of lung affected by interstitial pneumonia in high-resolution CT. Med. Phys., 35(12):5290-5302, 2008.

[364] B. Lassen, J.-M. Kuhnigk, O. Friman, S. Krass, and H.-O. Peitgen. Automatic segmentation of lung lobes in CT images based on fissures, vessels, and bronchi. In Proc. IEEE Int. Symp. Biomed. Imag.: From Nanon to Macro, (ISBI'10), pages 560563. IEEE, 2010.

[365] N. Birkbeck, T. Kohlberger, J. Zhang, M. Sofka, J. Kaftan, D. Comaniciu, and S. K. Zhou. Lung segmentation from CT with severe pathologies using anatomical constraints. Proc. Int. Conf. Medical Image Computing and Computer-Assisted Intervention, (MICCAI'14), 8673(1):804-811, 2014.

[366] K. Li, X. Wu, D. Z. Chen, and M. Sonka. Optimal surface segmentation in volumetric images-a graph-theoretic approach. IEEE Transactions on Pattern Analysis and Machine Intelligence, 28(1):119-134, 2006.

[367] J. K. Udupa and S. Samarasekera. Fuzzy connectedness and object definition: Theory, algorithms, and applications in image segmentation. Graphical Models and Image Processing, 58(3):246-261, 1996.

[368] B. Glocker, A. Sotiras, N. Komodakis, and N. Paragios. Deformable medical image registration: Setting the state of the art with discrete methods. Annual Review of Biomedical Engineering, 13:219-244, 2011. 
[369] T. Lindeberg. Generalized Gaussian scale-space axiomatics comprising linear scalespace, affine scale-space and spatio-temporal scale-space. J. Mathem. Imag. Vis., 40(1):36-81, 2011.

[370] A. Farag, A. El-Baz, and G. Gimel'farb. Precise segmentation of multimodal images. IEEE Trans. Image Processing, 15(4):952-968, 2006.

[371] G. Gimel'farb. Image Textures and Gibbs Random Fields. Kluwer Academic, 1999.

[372] B. Glocker, N. Komodakis, G. Tziritas, N. Navab, and N. Paragios. Dense image registration through MRFs and efficient linear programming. Medical Image Analysis, 12(6):731-741, 2008.

[373] B. Glocker, N. Komodakis, N. Paragios, and N. Navab. Non-rigid registration using discrete MRFs: Application to thoracic CT images. In Medical Image Analysis for the Clinic - A Grand Challenge, pages 147-154, 2010.

[374] Y. Lu, I. Cohen, X. S. Zhou, and Q. Tian. Feature selection using principal feature analysis. In Proceedings of the 15th ACM international conference on Multimedia, pages 301-304. ACM, 2007.

[375] A. El-Baz, A. Elnakib, F. Khalifa, M. A. El-Ghar, P. McClure, A. Soliman, and G. Gimel'farb. Precise segmentation of 3-D magnetic resonance angiography. IEEE Trans. on Biomedical Engineering, 59(7):2019-2029, 2012.

[376] K. H. Zou, S. K. Warfield, A. Bharatha, C. M. C. Tempany, M. R. Kaus, S. J. Haker, W. M. Wells III, F. A. Jolesz, and R. Kikinis. Statistical validation of image segmentation quality based on a spatial overlap index. Academic Radiology, 11(2):178-189, 2004.

[377] G. Gerig, M. Jomier, and M. Chakos. Valmet: A new validation tool for assessing and improving 3D object segmentation. In Medical Image Computing and Computer Assisted Intervention, Utrecht, The Netherlands, October 14-17,.

[378] R. D. Rudyanto, S. Kerkstra, E. M. Van Rikxoort, C. Fetita, P.-Y. Brillet, et al. Comparing algorithms for automated vessel segmentation in computed tomography scans of the lung: the vessel12 study. Medical Image Analysis, 18(7):1217-1232, 2014.

[379] M. S. Brown, M. F. Mcnitt-Gray, N. J. Mankovich, J. G. Goldin, J. Hiller, L. S. Wilson, and D. Aberie. Method for segmenting chest CT image data using an anatomical model: preliminary results. IEEE Trans. Med. Imaging, 16(6):828-839, 1997.

[380] B. Lassen, E. M. van Rikxoort, M. Schmidt, S. Kerkstra, B. van Ginneken, and J.-M. Kuhnigk. Automatic segmentation of the pulmonary lobes from chest ct scans based on fissures, vessels, and bronchi. IEEE Trans. Med. Imaging, 32(2):210-222, 2013.

[381] O. Weinheimer, T. Achenbach, C. P. Heusse, and C. Düber. Automatic lung segmentation in MDCT images. LObe and Lung Analysis (LOLA11) MICCAI Challeng . [Online]. Available: http: / / lola11.com/Results/Overview, 2011.

[382] F. Malmberg, R. Nordenskjöld, R. Strand, and J. Kullberg. Smartpaint: A tool for interactive segmentation of medical volume images. Comput. Methods Biomech. Biomed. Eng.: Imaging \& Visual., (ahead-of-print):1-9, 2014. 
[383] A. Fernandez-Bustamante, J. Klawitter, J. E. Repine, A. Agazio, A. J. Janocha, et al. Early effect of tidal volume on lung injury biomarkers in surgical patients with healthy lungs. Anesthesiology, 121(3):469-481, 2014.

[384] A. Soliman, F. Khalifa, A. Elnakib, M. A. El-Ghar, N. Dunlap, B. Wang, G. Gimel'farb, R. Keynton, and A. El-Baz. Accurate lungs segmentation on ct chest images by adaptive appearance-guided shape modeling. IEEE Transactions on Medical Imaging, 2016.

[385] A. Soliman, F. Khalifa, A. Alansary, G. Gimel'farb, and A. El-Baz. Performance evaluation of an automatic MGRF-based lung segmentation approach. In Proc. Intern. Symp. on Computational Models for Life Sciences (CMLS'2013), volume 1559, pages 323-332, 2013.

[386] A. Soliman, F. Khalifa, A. Alansary, G. Gimel'farb, and A. El-Baz. Segmentation of lung region based on using parallel implementation of joint MGRF: Validation on 3D realistic lung phantoms. In Proc. Intern. Symp. on Biomedical Imaging: From Nano to Macro (ISBI'13), pages 864-867. IEEE Press, 2013.

[387] B. Abdollahi, A. Soliman, A. C. Civelek, X.-F. Li, G. Gimel'farb, and A. El-Baz. A novel Gaussian scale space-based joint MGRF framework for precise lung segmentation. In Proc. IEEE Intern. Conf. on Image Processing (ICIP'12), pages 2029-2032. IEEE Press, 2012.

[388] A. Soliman, A. Elnakib, F. Khalifa, M. A. El-Ghar, and A. El-Baz. Segmentationof pathological lungs from ct chest images. In Image Processing (ICIP), 2015 IEEE International Conference on, pages 3655-3659. IEEE, 2015.

[389] E. Castillo, R. Castillo, J. Martinez, M. Shenoy, and T. Guerrero. Four-dimensional deformable image registration using trajectory modeling. Physics in Medicine and Biology, 55(1):305, 2010.

[390] S. Schaefer, T. McPhail, and J. Warren. Image deformation using moving least squares. 25(3):533-540, 2006.

[391] Y. Zheng, K. Steiner, T. Bauer, J. Yu, D. Shen, and C. Kambhamettu. Lung nodule growth analysis from $3 \mathrm{~d}$ ct data with a coupled segmentation and registration framework. In IEEE 11th International Conference on Computer Vision (ICCV'2007), pages 1-8. IEEE, 2007.

[392] C. Metz, S. Klein, M. Schaap, T. van Walsum, and W. J. Niessen. Non rigid registration of dynamic medical imaging data using $\mathrm{nD}+\mathrm{t} \mathrm{B}$-splines and a group wise optimization approach. Medical image analysis, 15(2):238-249, 2011.

[393] H. Heinrich, M. Jenkinson, M. Brady, and J. A. Schnabel. MRF-based deformable registration and ventilation estimation of lung CT. Medical Imaging, IEEE Transactions on, 32(7):1239-1248, 2013.

[394] L. Konig and J. Ruhaak. A fast and accurate parallel algorithm for non-linear image registration using normalized gradient fields. In Biomedical Imaging (ISBI), 2014 IEEE 11th International Symposium on, pages 580-583. IEEE, 2014.

[395] P. A. Viola and W. M. W. III. Alignment by maximization of mutual information. Int. J. Comput. Vis., 24(2):137-154, 1997. 
[396] D. Adalsteinsson and J. A. Sethian. A fast level set method for propagating interfaces. Journal of computational physics, 118(2):269-277, 1995.

[397] S. Haker et al. Nondistorting flattening maps and the 3D visualization of colon CT images. IEEE Trans. Med. Imag., 19(7):665-670, 2000.

[398] F. Khalifa, G. M. Beache, G. Gimel'farb, G. A. Giridharan, and A. El-Baz. Accurate automatic analysis of cardiac cine images. IEEE Trans. Biomedical Engineering, 59(2):445-455, 2012.

[399] M. Prasad et al. Automated quantification of 3D regional myocardial wall thickening from gated magnetic resonance images. J. Magn. Reson. Imaging, 31(2):317-327, 2010.

[400] C. Bouman and K. Sauer. A generalized Gaussian image model for edge-preserving MAP estimation. IEEE Trans. Image Processing, 2(3):296-310, 1993.

[401] J. Besag. On the statistical analysis of dirty pictures. Journal of the Royal Statistical Society. Series B (Methodological), pages 259-302, 1986.

[402] D. Rueckert, L. I. Sonoda, C. Hayes, D. L. Hill, M. O. Leach, and D. J. Hawkes. Nonrigid registration using free-form deformations: application to breast mr images. Medical Imaging, IEEE Transactions on, 18(8):712-721, 1999.

[403] R. Castillo, E. Castillo, R. Guerra, V. E. Johnson, T. McPhail, A. K. Garg, and T. Guerrero. A framework for evaluation of deformable image registration spatial accuracy using large landmark point sets. Physics in medicine and biology, 54(7):1849, 2009.

[404] H. Wang, L. Dong, J. O’Daniel, R. Mohan, A. S. Garden, K. K. Ang, D. A. Kuban, M. Bonnen, J. Y. Chang, and R. Cheung. Validation of an accelerated demons algorithm for deformable image registration in radiation therapy. Physics in medicine and biology, 50(12):2887, 2005.

[405] A. Soliman, F. Khalifa, N. Dunlap, B. Wang, M. A. El-Ghar, and A. El-Baz. An isosurfaces based local deformation handling framework of lung tissues. In Biomedical Imaging (ISBI), 2016 IEEE 13th International Symposium on, pages 1253-1259. IEEE, 2016.

[406] A. R. Larici et al. Lung abnormalities at multimodality imaging after radiation therapy for non-small cell lung cancer. Radiographics, 31(3):771-789, 2011.

[407] K. Latifi et al. Impact of dose on lung ventilation change calculated from 4D-CT using deformable image registration in lung cancer patients treated with SBRT. Journal of Radiation Oncology, 4(3):265-270, 2015.

[408] T. Yamamoto et al. Evaluation of four-dimensional 4D computed tomography CT pulmonary ventilation imaging by comparison with single photon emission computed tomography SPECT scans for a lung cancer patient. In Third Int. Workshop Pul. Image Anal., MICCAI, Beijing, China, pages 117-128, 2010.

[409] J. M. Reinhardt et al. Registration-based estimates of local lung tissue expansion compared to xenon CT measures of specific ventilation. Med. image anal., 12(6):752-763, 2008. 
[410] Y. Vinogradskiy et al. Use of 4-dimensional computed tomography-based ventilation imaging to correlate lung dose and function with clinical outcomes. Int. J. Radiat. Oncol. Biol. Phys., 86(2):366-371, 2013.

[411] W. Zhang et al. Quantitative study of lung perfusion SPECT scanning and pulmonary function testing for early radiation-induced lung injury in patients with locally advanced non-small cell lung cancer. Exp. Ther. Med., 3.

[412] H. Sharifi, W. Van Elmpt, C. Oberije, G. Nalbantov, M. Das, M. Öllers, P. Lambin, A.-M. C. Dingmans, and D. De Ruysscher. Quantification of ct-assessed radiationinduced lung damage in lung cancer patients treated with or without chemotherapy and cetuximab. Acta Oncologica, 55(2):156-162, 2016.

[413] M. Negahdar and A. A. Amini. Regional lung strains via a volumetric mass conserving optical flow model. In 2012 9th IEEE International Symposium on Biomedical Imaging (ISBI), pages 1475-1478. IEEE, 2012.

[414] M. Negahdara, A. Zacariasb, R. A. Milamc, N. Dunlapb, S. Y. Woob, and A. A. Aminia. An automated landmark-based elastic registration technique for large deformation recovery from 4-d ct lung images. In Proc. of SPIE Vol, volume 8317, pages $83171 \mathrm{G}-1,2012$.

[415] M. Negahdar and A. A. Amini. A 3d optical flow technique based on mass conservation for deformable motion estimation from 4-d ct images of the lung. In SPIE Medical Imaging, pages 83171F-83171F. International Society for Optics and Photonics, 2012.

[416] M. Negahdar and A. A. Amini. Tracking planar lung motion in 4d ct with optical flow: validations and comparison of global, local, and local-global methods. In SPIE Medical Imaging, pages 762623-762623. International Society for Optics and Photonics, 2010.

[417] M. Negahdar, N. Dunlap, A. Zacarias, A. C. Civelek, S. Y. Woo, and A. A. Amini. Comparison of indices of regional lung function from 4-D X-ray CT: Jacobian vs. strain of deformation. In 2013 IEEE 10th International Symposium on Biomedical Imaging, pages 648-651. IEEE, 2013.

[418] A. Blake, P. Kohli, and C. Rother. Markov random fields for vision and image processing. Mit Press, 2011.

[419] G. Gimel'farb and D. Zhou. Texture analysis by accurate identification of a generic Markov-Gibbs model. In Applied Pattern Recognition, pages 221-245. Springer, 2008.

[420] N. Liu, G. Gimel'farb, and P. Delmas. High-order MGRF models for contrast/offset invariant texture retrieval. In Proc. Int. Conf. Image Vision Computing New Zealand, pages 96-101. ACM, 2014.

[421] V. Vishnevskiy, T. Gass, G. Szekely, C. Tanner, and O. Goksel. Isotropic total variation regularization of displacements in parametric image registration. IEEE Transactions on Medical Imaging, 2016. 
[422] D. Mahapatra. Analyzing training information from random forests for improved image segmentation. Image Processing, IEEE Transactions on, 23(4):1504-1512, 2014.

[423] T. K. Ho. Random decision forests. In Proceedings of the Third International Conference on Document Analysis and Recognition, 1995.,, volume 1, pages 278-282. IEEE, 1995.

[424] L. Breiman. Random forests. Machine learning, 45(1):5-32, 2001.

[425] N. Liu, A. Soliman, G. Gimelfarb, and A. El-Baz. Segmenting kidney dce-mri using 1st-order shape and 5th-order appearance priors. In International Conference on Medical Image Computing and Computer-Assisted Intervention, pages 77-84. Springer, 2015.

[426] A. Soliman, F. Khalifa, A. Shaffie, N. Dunlap, B. Wang, A. Elmaghraby, and A. ElBaz. Detection of lung injury using 4d-ct chest images. In Biomedical Imaging (ISBI), 2016 IEEE 13th International Symposium on, pages 1274-1277. IEEE, 2016.

[427] A. Sotiman, F. Khalifa, A. Shaffie, N. Liu, N. Dunlap, B. Wang, A. Elmaghraby, G. Gimel'farb, and A. El-Baz. Image-based cad system for accurate identification of lung injury. In Image Processing (ICIP), 2016 IEEE International Conference on, pages 121-125. IEEE, 2016.

[428] A. Elnakib, M. F. Casanova, A. Soliman, G. Gimelfarb, and A. El-Baz. Analysis of $3 \mathrm{~d}$ corpus callosum images in the brains of autistic individuals. Handbook of Research on Trends in the Diagnosis and Treatment of Chronic Conditions, page 159, 2015.

[429] A. Elnakib, A. Soliman, M. Nitzken, M. F. Casanova, G. Gimel'farb, and A. ElBaz. Magnetic resonance imaging findings for dyslexia: A review. J. Biomedical Nanotechnology, 10(10):2778-2805, 2014.

[430] M. Ismail, R. Keynton, M. Mostapha, A. ElTanboly, M. Casanova, G. Gimel'farb, and A. El-Baz. Studying autism spectrum disorder with structural and diffusion magnetic resonance imaging: A survey. Frontiers in Human Neuroscience, 10, 2016.

[431] M. Ismail, A. Soliman, A. ElTanboly, A. Switala, M. Mahmoud, F. Khalifa, G. Gimel'farb, M. F. Casanova, R. Keynton, and A. El-Baz. Detection of white matter abnormalities in $\mathrm{mr}$ brain images for diagnosis of autism in children. In Proceedings of IEEE 13th International Symposium on Biomedical Imaging (ISBI'16), pages 6-9. IEEE, 2016.

[432] A. Alansary, M. Ismail, A. Soliman, F. Khalifa, M. Nitzken, et al. Infant brain extraction in t1-weighted $\mathrm{mr}$ images using bet and refinement using lcdg and mgrf models. IEEE journal of biomedical and health informatics, 20(3):925-935, 2016.

[433] M. F. Casanova, A. E.-B. S, S. S. Kamat, B. A. Dombroski, F. Khalifa, A. Elnakib, A. Soliman, A. Allison-McNutt, and A. E. Switala. Focal cortical dysplasias in autism spectrum disorders. Acta Neuropathologica Communications, 1(1):67, 2013.

[434] A. Alansary, A. Soliman, F. Khalifa, A. Elnakib, M. Mostapha, M. Nitzken, M. Casanova, and A. El-Baz. MAP-based framework for segmentation of mr brain images based on visual appearance and prior shape. MIDAS J, 1:1, 2013. 
[435] M. Ismail, M. Mostapha, A. Soliman, M. Nitzken, F. Khalifa, A. Elnakib, G. Gimel'farb, M. Casanova, and A. El-Baz. Segmentation of infant brain mr images based on adaptive shape prior and higher-order mgrf. In Image Processing (ICIP), 2015 IEEE International Conference on, pages 4327-4331. IEEE, 2015.

[436] A. Alansary, A. Soliman, M. Nitzken, F. Khalifa, A. Elnakib, M. Mostapha, M. F. Casanova, and A. El-Baz. An integrated geometrical and stochastic approach for accurate infant brain extraction. In Proc. 2014 IEEE Intern. Conf. on Image Processing (ICIP 2014), pages 3542-3546. IEEE Press, 2014.

[437] B. A. Dombroski, A. E. Switala, A. S. El-Baz, and M. F. Casanova. Gyral window mapping of typical cortical folding using MRI. Translational Neuroscience, 2(2):142-147, 2011.

[438] B. Dombroski, M. Nitzken, A. Elnakib, F. Khalifa, A. El-Baz, and M. F. Casanova. Cortical surface complexity in a population-based normative sample. Translational Neuroscience, 5(1):17-24, 2014.

[439] A. El-Baz, M. Casanova, G. Gimel'farb, M. Mott, and A. Switala. An MRI-based diagnostic framework for early diagnosis of dyslexia. Intern. J. Computer Assisted Radiology and Surgery, 3(3-4):181-189, 2008.

[440] A. El-Baz, M. Casanova, G. Gimel'farb, M. Mott, A. Switala, E. V. Bogaert, and R. McCracken. A new CAD system for early diagnosis of dyslexic brains. In Proc. IEEE Intern. Conf. on Image Processing (ICIP'2008), pages 1820-1823, 2008.

[441] A. El-Baz, M. F. Casanova, G. Gimel'farb, M. Mott, and A. E. Switala. Autism diagnostics by 3D texture analysis of cerebral white matter gyrifications. In Proc. Intern. Conf. on Medical Image Computing and Computer-Assisted Intervention (MICCAI'2007), pages 882-890. Springer, 2007.

[442] A. El-Baz, M. F. Casanova, G. Gimel'farb, M. Mott, and A. E. Switala. A new image analysis approach for automatic classification of autistic brains. In Proc. IEEE Intern. Symp. on Biomedical Imaging: From Nano to Macro (ISBI'2007), pages 352-355. IEEE Press, 2007.

[443] A. El-Baz, A. A. Farag, G. L. Gimel'farb, M. A. El-Ghar, and T. Eldiasty. Probabilistic modeling of blood vessels for segmenting MRA images. In Proc. $18^{\text {th }}$ IAPR Intern. Conf. on Pattern Recognition (ICPR'06), volume 3, pages 917-920, Hong Kong, China, Aug. 20-24, 2006. IEEE Press.

[444] A. El-Baz, A. A. Farag, G. Gimel'farb, M. A. El-Ghar, and T. Eldiasty. A new adaptive probabilistic model of blood vessels for segmenting MRA images. In Proc. Intern. Conf. on Medical Image Computing and Computer-Assisted Intervention (MICCAI 2006), volume 4191, pages 799-806. Springer, 2006.

[445] A. El-Baz, A. Farag, G. Gimelfarb, and S. Hushek. Automatic cerebrovascular segmentation by accurate probabilistic modeling of tof-mra images. In Medical Image Computing and Computer-Assisted Intervention (MICCAI'05), pages 34-42. Springer, 2005. 
[446] A. El-Baz, A. Elnakib, M. F. Casanova, G. Gimel'farb, A. E. Switala, D. Jordan, and S. Rainey. Accurate automated detection of autism related Corpus Callosum abnormalities. J. Medical Systems, 35(5):929-939, 2011.

[447] A. El-Baz, M. Casanova, G. Gimel'farb, M. Mott, A. Switala, E. V. Bogaert, and R. McCracken. Dyslexia diagnostics by 3D texture analysis of cerebral white matter gyrifications. In Proc. IAPR Intern. Conf. on Pattern Recognition (ICPR'2008), pages $1-4,2008$.

[448] A. El-Baz, M. Casanova, G. Gimel'farb, M. Mott, and A. Switala. A new imagebased diagnostic framework for early diagnosis of dyslexic brains. In Proc. $22^{\text {nd }}$ Intern. Congress and Exhibition on Computer Assisted Radiology and Surgery (CARS'08), pages 46-47, Barcelona, Spain, June 25-28, 2008.

[449] A. Elnakib, A. El-Baz, M. F. Casanova, G. Gimel'farb, and A. E. Switala. Imagebased detection of corpus callosum variability for more accurate discrimination between dyslexic and normal brains. In Proc. IEEE Intern. Symp. on Biomedical Imaging: From Nano to Macro (ISBI'2010), pages 109-112, 2010.

[450] A. Elnakib, A. El-Baz, M. F. Casanova, G. Gimel'farb, and A. E. Switala. Imagebased detection of Corpus Callosum variability for more accurate discrimination between autistic and normal brains. In Proc. IEEE Intern. Conf. on Image Processing (ICIP2010), pages 4337-4340, 2010.

[451] A. Elnakib, A. El-Baz, M. F. Casanova, and A. E. Switala. Dyslexia diagnostics by centerline-based shape analysis of the corpus callosum. In Proc. IAPR Intern. Conf. on Pattern Recognition (ICPR'2010), pages 261-264, 2010.

[452] A. Elnakib, M. F. Casanova, G. Gimel'farb, A. E. Switala, and A. El-Baz. Dyslexia diagnostics by 3-D shape analysis of the corpus callosum. IEEE Trans. Information Technology in Biomedicine, 16(4):700-708, 2012.

[453] A. Elnakib, M. F. Casanova, G. Gimel'farb, A. E. Switala, and A. El-Baz. Autism diagnostics by centerline-based shape analysis of the Corpus Callosum. In Proc. IEEE Intern. Symp. on Biomedical Imaging: From Nano to Macro (ISBI'2011), pages 1843-1846, 2011.

[454] A. Elnakib, M. F. Casanova, G. Gimel'farb, and A. El-Baz. Autism diagnostics by 3D shape analysis of the Corpus Callosum. In K. Suzuki, editor, Machine Learning in Computer-aided Diagnosis: Medical Imaging Intelligence and Analysis, chapter 15, pages 315-335. IGI Global, 2012.

[455] R. Fahmi, A. El-Baz, H. Hassan, A. A. Farag, and M. F. Casanova. Structural MRIbased discrimination between autistic and typically developing brain. In Proc. Computer Assisted Radiology and Surgery (CARS'2007), pages 24-26, 2007.

[456] M. Mostapha, A. Alansary, A. Soliman, F. Khalifa, M. Nitzken, R. Khodeir, M. F. Casanova, and A. El-Baz. Atlas-based approach for the segmentation of infant DTI MR brain images. In Proc. $11^{\text {th }}$ IEEE Intern. Symp. on Biomedical Imaging (ISBI 2014), pages 1255-1258. IEEE Press, 2014.

[457] M. Mostapha, A. Soliman, F. Khalifa, A. Elnakib, A. Alansary, M. Nitzken, M. F. Casanova, and A. El-Baz. A statistical framework for the classification of infant DT 
images. In Proc. $21^{\text {st }}$ IEEE Intern. Conf. on Image Processing (ICIP 2014), pages 2222-2226, Paris, France, Oct. 27-30, 2014. IEEE Press.

[458] M. Nitzken, M. F. Casanova, G. Gimel'farb, A. Elnakib, F. Khalifa, A. Switala, and A. El-Baz. 3D shape analysis of the brain cortex with application to dyslexia. In Proc. $18^{\text {th }}$ IEEE Intern. Conf. on Image Processing (ICIP 2011), pages 2657-2660, Brussels, Belgium, Sept. 11-14, 2011. IEEE Press.

[459] M. Nitzken, M. F. Casanova, G. Gimel'farb, F. Khalifa, A. Elnakib, A. E. Switala, and A. El-Baz. 3D shape analysis of the brain cortex with application to autism. In Proc. $8^{\text {th }}$ IEEE Intern. Symp. on Biomedical Imaging: From Nano to Macro (ISBI 2011), pages 1847-1850, Chicago, IL, USA, March 30 - April 2, 2011. IEEE Press.

[460] M. Nitzken, M. F. Casanova, F. Khalifa, G. Sokhadze, and A. El-Baz. Shape-based detection of cortex variability for more accurate discrimination between autistic and normal brains. In A. El-Baz., R. Acharya, A. Laine, and J. Suri, editors, Handbook of Multi-Modality State-of-the-Art Medical Image Segmentation and Registration Methodologies, volume 2, chapter 7, pages 161-185. Springer, New York, 2011.

[461] M. J. Nitzken, M. F. Casanova, and A. El-Baz. SPHARM analysis of the brain cortex for diagnosing dyslexia. In Proc. IEEE $11^{\text {th }}$ Intern. Symp. on Biomedical Imaging (ISBI 2014), Beijing, China, April 29 - May 2, 2014.

[462] M. J. Nitzken, M. F. Casanova, G. Gimelfarb, T. Inanc, J. M. Zurada, and A. El-Baz. Shape analysis of the human brain: A brief survey. IEEE J. Biomedical and Health Informatics, 18(4):1337-1354, 2014.

[463] A. Elnakib, G. M. Beache, G. Gimelfarb, and A. El-Baz. Intramyocardial strain estimation from cardiac cine mri. International journal of computer assisted radiology and surgery, 10(8):1299-1312, 2015.

[464] G. M. Beache, F. Khalifa, G. Gimel'farb, and A. El-Baz. Fully automated framework for the analysis of myocardial first-pass perfusion MR images. Medical Physics, 41(10):1-18, 2014.

[465] A. Elnakib, G. M. Beache, G. Gimelfarb, and A. El-Baz. New automated MarkovGibbs random field based framework for myocardial wall viability quantification on agent enhanced cardiac magnetic resonance images. Int. J. Cardiovasc. Imaging, 28(7):1683-1698, 2012.

[466] A. Elnakib, G. M. Beache, G. Gimel'farb, and A. El-Baz. A new framework for automated segmentation of left ventricle wall from contrast enhanced cardiac magnetic resonance images. In Proc. IEEE Intern. Conf. on Image Processing (ICIP'2011), pages 2289-2292, 2011.

[467] A. Elnakib, G. M. Beache, T. I. G. Gimel'farb, and A. El-Baz. Validating a new methodology for strain estimation from cardiac cine MRI. In Proc. Intern. Symp. on Computational Models for Life Science (CMLS'13), pages 277-286, 2013.

[468] H. Sliman, F. Khalifa, A. Elnakib, A. Soliman, G. Beache, G. Gimel'farb, A. Emam, A. Elmaghraby, and A. El-Baz. Accurate segmentation framework for the left ventricle wall from cardiac cine mri. In 2013 INTERNATIONAL SYMPOSIUM ON COM- 
PUTATIONAL MODELS FOR LIFE SCIENCES, volume 1559, pages 287-296. AIP Publishing, 2013.

[469] A. Elnakib, G. M. Beache, M. Nitzken, G. Gimel'farb, and A. El-Baz. A new framework for automated identification of pathological tissues in contrast enhanced cardiac magnetic resonance images. In Proc. IEEE Intern. Symp. on Biomedical Imaging: From Nano to Macro (ISBI'2011), pages 1272-1275, 2011.

[470] A. Elnakib, G. Beache, H. Sliman, G. Gimel'farb, T. Inanc, and A. El-Baz. A novel Laplace-based method to estimate the strain from cine cardiac magnetic resonance images. In Proceedings of IEEE International Conference on Image Processing (ICIP'2013). IEEE, 2013.

[471] F. Khalifa, G. Beache, A. El-Baz, and G. Gimel'farb. Deformable model guided by stochastic speed with application in cine images segmentation. In Proc. IEEE Intern. Conf. on Image Processing (ICIP'2010), pages 1725-1728, Hong Kong, September 26-29, 2010.

[472] F. Khalifa, G. M. Beache, A. Elnakib, H. Sliman, G. Gimel'farb, K. C. Welch, and A. El-Baz. A new shape-based framework for the left ventricle wall segmentation from cardiac first-pass perfusion MRI. In Proc. IEEE Int. Symp. Biomed. Imaging: From Nano to Macro, (ISBI'13), pages 41-44, 2013.

[473] F. Khalifa, G. M. Beache, A. Elnakib, H. Sliman, G. Gimel'farb, K. C. Welch, and A. El-Baz. A new nonrigid registration framework for improved visualization of transmural perfusion gradients on cardiac first-pass perfusion MRI. In Proc. IEEE Int. Symp. Biomed. Imaging: From Nano to Macro, (ISBI'12), pages 828-831, 2012.

[474] F. Khalifa, G. M. Beache, A. Firjani, K. C. Welch, G. Gimel'farb, and A. El-Baz. A new nonrigid registration approach for motion correction of cardiac first-pass perfusion MRI. In Proc. IEEE Int. Conf. Image Process., (ICIP'12), pages 1665-1668, 2012.

[475] F. Khalifa, G. M. Beache, G. Gimel'farb, and A. El-Baz. A novel CAD system for analyzing cardiac first-pass MRI images. In Proc. IEEE Int. Conf. Pattern Recogni., (ICPR'12), pages 77-80, 2012.

[476] F. Khalifa, G. M. Beache, G. Gimel'farb, and A. El-Baz. A novel approach for accurate estimation of left ventricle global indexes from short-axis cine MRI. In Proc. IEEE Intern. Conf. on Image Processing (ICIP'2011), pages 2645-2649, Brussels, Belgium, September 11-14, 2011.

[477] F. Khalifa, G. M. Beache, G. Gimel'farb, G. A. Giridharan, and A. El-Baz. A new image-based framework for analyzing cine images. In A. El-Baz, U. R. Acharya, M. Mirmedhdi, and J. S. Suri, editors, Handbook of Multi Modality State-of-the-Art Medical Image Segmentation and Registration Methodologies, volume 2, chapter 3, pages 69-98. Springer, 2011.

[478] F. Khalifa, G. M. Beache, G. Gimel'farb, G. A. Giridharan, and A. El-Baz. Accurate automatic analysis of cardiac cine images. IEEE Trans. Biomed. Eng., 59(2):445$455,2012$. 
[479] F. Khalifa, G. M. Beache, M. Nitzken, G. Gimel'farb, G. A. Giridharan, and A. ElBaz. Automatic analysis of left ventricle wall thickness using short-axis cine CMR images. In Proc. IEEE Int. Symp. Biomed. Imaging: From Nano to Macro, (ISBI'11), pages 1306-1309, 2011.

[480] M. Nitzken, G. Beache, A. Elnakib, F. Khalifa, G. Gimel'farb, and A. El-Baz. Accurate modeling of tagged CMR 3D image appearance characteristics to improve cardiac cycle strain estimation. In Proc. $19^{\text {th }}$ IEEE Intern. Conf. on Image Processing (ICIP 2012), pages 521-524, Orlando, FL, USA, Sept. 30 - Oct. 3, 2012. IEEE Press.

[481] M. J. Nitzken, A. S. El-Baz, and G. M. Beache. Markov-Gibbs random field model for improved full-cardiac cycle strain estimation from tagged CMR. J. Cardiovascular Magnetic Resonance, 14(1):1-2, 2012.

[482] H. Sliman, A. Elnakib, G. M.Beache, A. Elmaghraby, and A. El-Baz. Assessment of myocardial function from cine cardiac MRI using a novel 4D tracking approach. J. Computer Science \& Systems Biology, 7:169-173, 2014.

[483] H. Sliman, A. Elnakib, G. M. Beache, A. Soliman, F. Khalifa, G. Gimel'farb, A. Elmaghraby, and A. El-Baz. A novel 4D PDE-based approach for accurate assessment of myocardium function using cine cardiac magnetic resonance images. In Proc. $21^{\text {st }}$ IEEE Intern. Conf. on Image Processing (ICIP 2014), pages 3537-3541, Paris, France, October 27-30, 2014.

[484] H. Sliman, F. Khalifa, A. Elnakib, A. Soliman, G. M. Beache, A. Elmaghraby, G. Gimel'farb, and A. El-Baz. Myocardial borders segmentation from cine MR images using bi-directional coupled parametric deformable models. Medical Physics, 40(9):1-13, 2013.

[485] H. Sliman, F. Khalifa, A. Elnakib, G. Beache, A. Elmaghraby, and A. El-Baz. A new segmentation-based tracking framework for extracting the left ventricle cavity from cine cardiac MRI. In Proceedings of IEEE International Conference on Image Processing, (ICIP'13), pages 685-689, Melbourne, Australia, September 15-18, 2013.

[486] H. Sliman, F. Khalifa, A. Elnakib, A. Soliman, G. M. Beache, G. Gimel'farb, A. Emam, A. Elmaghraby, and A. El-Baz. Accurate segmentation framework for the left ventricle wall from cardiac cine MRI. In AIP Proc.: Intern. Symp. on Computational Models for Life Science, (CMLS'13), volume 1559, pages 287-296, Sydney, Australia, Nov. 27-29, 2013.

[487] A. Firjani, A. Elmaghraby, and A. El-Baz. MRI-based diagnostic system for early detection of prostate cancer. In Proc. Biomedical Sciences and Engineering Conf. (BSEC 2013), pages 1-4, 2013.

[488] A. Firjani, A. Elnakib, F. Khalifa, A. El-Baz, G. Gimel'farb, M. A. El-Ghar, and A. Elmaghraby. A novel 3D segmentation approach for segmenting the prostate from dynamic contrast enhanced MRI using current appearance and learned shape prior. In Proc. IEEE Intern. Symp. on Signal Processing and Information Technology, (ISSPIT'10), pages 137-143, Luxor, Egypt, December 15-18, 2010.

[489] P. McClure, A. Elnakib, M. A. El-Ghar, F. Khalifa, A. Soliman, T. El-Diasty, J. S. Suri, A. Elmaghraby, and A. El-Baz. In-vitro and in-vivo diagnostic techniques for 
prostate cancer: A review. Journal of biomedical nanotechnology, 10(10):27472777, 2014.

[490] A. Firjani, A. Elnakib, F. Khalifa, G. Gimel'farb, M. A. El-Ghar, J. Suri, A. Elmaghraby, and A. El-Baz. A new 3D automatic segmentation framework for accurate extraction of prostate from DCE-MRI. In Proc. IEEE Intern. Symp. on Biomedical Imaging: From Nano to Macro (ISBI'11), pages 1476-1479, Chicago, Illinois, March 30-April 2, 2011.

[491] A. Firjani, A. Elnakib, F. Khalifa, G. Gimel'farb, M. Abou El-Ghar, A. Elmaghraby, and A. El-Baz. A diffusion-weighted imaging based diagnostic system for early detection of prostate cancer. J. Biomed. Sci. Eng., 6(3A):346-356, 2013.

[492] A. Firjani, F. Khalifa, A. Elnakib, G. Gimel'farb, M. A. El-Ghar, A. Elmaghraby, and A. El-Baz. Non-invasive image-based approach for early detection of prostate cancer. In Proc. Fourth Intern. Conf. on Developments in eSystems Engineering (DeSE'11), pages 172-177, Dubai, UAE, December 6-8, 2011.

[493] A. Firjani, F. Khalifa, A. Elnakib, G. Gimel'farb, M. A. El-Ghar, A. Elmaghraby, and A. El-Baz. 3D automatic approach for precise segmentation of the prostate from diffusion-weighted magnetic resonance imaging. In Proc. IEEE Intern. Conf. on Image Processing (ICIP'11), pages 2285-2288, Brussels, Belgium, September 11-14, 2011.

[494] A. Firjani, F. Khalifa, A. Elnakib, G. Gimel'farb, M. A. El-Ghar, A. Elmaghraby, and A. El-Baz. A novel image-based approach for early detection of prostate cancer using DCE-MRI. In K. Suzuki, editor, Computational Intelligence in Biomedical Imaging, pages 55-82. Springer, 2014.

[495] A. Firjani, F. Khalifa, A. Elnakib, G. Gimel'farb, A. Elmaghraby, and A. El-Baz. A novel image-based approach for early detection of prostate cancer. In Proc. IEEE Intern. Conf. on Image Processing (ICIP'12), pages 2849-2852, Lake Buena Vista, Florida, Sept. 30-Oct. 3, 2012.

[496] I. Reda, A. Shalaby, M. A. El-Ghar, F. Khalifa, M. Elmogy, A. Aboulfotouh, E. Hosseini-Asl, A. El-Baz, and R. Keynton. A new nmf-autoencoder based cad system for early diagnosis of prostate cancer. In Biomedical Imaging (ISBI), 2016 IEEE 13th International Symposium on, pages 1237-1240. IEEE, 2016.

[497] A. Firjany, A. Elnakib, A. El-Baz, G. Gimel'farb, M. El-Ghar, and A. Elmagharby. Novel stochastic framework for accurate segmentation of prostate in dynamic contrast enhanced MRI. In A. Madabhushi, J. Dowling, P. Yan, A. Fenster, P. Abolmaesumi, and N. Hata, editors, Prostate Cancer Imaging. Computer-Aided Diagnosis, Prognosis, and Intervention, volume 6367 of Lecture Notes in Computer Science, pages 121-130. Springer, 2010.

[498] A. Firjany, A. Elnakib, A. El-Baz, G. Gimel'farb, M. A. El-Ghar, and A. Elmagharby. Novel stochastic framework for accurate segemntation of prostate in dynamic contrast-enhanced MRI. In Proc. Intern. Wokshop on Prostate Cancer Imaging: Computer-Aided Diagnosis, Prognosis, and Intervention, pages 121-130, Beijing, China, Sept. 24, 2010. 
[499] P. McClure, F. Khalifa, A. Soliman, M. A. El-Ghar, G. Gimelfarb, A. Elmagraby, and A. El-Baz. A novel NMF guided level-set for DWI prostate segmentation. $J$. Computer Science and Systems Biology, 7:209-216, 2014.

[500] A. Firjani, A. Elnakib, F. Khalifa, G. Gimel'farb, M. A. El-Ghar, A. Elmaghraby, and A. El-Baz. A new 3D automatic segmentation framework for accurate extraction of prostate from diffusion imaging. In Proc. Biomedical Science and Engineering Conf.- Image Informatics and Analytics in Biomedicine (BSEC'11), pages 13061309, Knoxville, Tennessee, March 15-17, 2011.

[501] M. Shehata, F. Khalifa, A. Soliman, M. Abou El-Ghar, A. Dwyer, R. Ouseph, and A. El-Baz. Early assessment of acute renal rejection. In 12th Annual Scientific Meeting of American Society for Diagnostics and Interventional Nephrology (ASDIN), Phoenix, Arizona, USA, Feb 19-21, 2016.

[502] M. Shehata, F. Khalifa, A. Soliman, A. Taki Eldeen, M. Abou El-Ghar, T. Eldiasty, A. El-Baz, and R. Keynton. An appearance-guided deformable model for 4D kidney segmentation using diffusion MRI. In X. J. A. El-Baz and T. . F. J. Suri, Eds, editors, Biomedical Image Segmentation: Advances and Trends, page (In Press). CRC, 2016.

[503] M. Shehata, F. Khalifa, A. Soliman, R. Alrefai, M. Abou El-Ghar, A. C. Dwyer, R. Ouseph, and A. El-Baz. A level set-based framework for 3D kidney segmentation from diffusion MR images. In Proceedings of IEEE 22nd International Conference on Image Processing, (ICIP'15), pages 4441-4445. IEEE, 2015.

[504] M. Mostapha, F. Khalifa, A. Alansary, A. Soliman, G. Gimel'farb, and A. El-Baz. Dynamic MRI-based computer aided diagnostic systems for early detection of kidney transplant rejection: A survey. In AIP Proc.: Intern. Symp. on Computational Models for Life Sciences (CMLS’2013), volume 1559, pages 297-306. AIP, 2013.

[505] A. K. Rudra, A. S. Chowdhury, A. Elnakib, F. Khalifa, A. Soliman, G. Beache, and A. El-Baz. Kidney segmentation using graph cuts and pixel connectivity. Pattern Recognition Letters, 34(13):1470-1475, 2013.

[506] M. Shehata, F. Khalifa, A. Soliman, R. Alrefai, M. Abou El-Ghar, A. C. Dwyer, R. Ouseph, and A. El-Baz. A novel framework for automatic segmentation of kidney from DW-MRI. In Proceedings of IEEE 12th International Symposium on Biomedical Imaging, (ISBI'15), pages 951-954. IEEE, 2015.

[507] M. Shehata, F. Khalifa, A. Soliman, M. A. El-Ghar, A. Dwyer, G. Gimelfarb, R. Keynton, and A. El-Baz. A promising non-invasive cad system for kidney function assessment. In International Conference on Medical Image Computing and Computer-Assisted Intervention, pages 613-621. Springer, 2016.

[508] F. Khalifa, A. Soliman, A. C. Dwyer, G. Gimel'farb, and A. El-Baz. A random forest-based framework for 3d kidney segmentation from dynamic contrastenhanced ct images. In Image Processing (ICIP), 2016 IEEE International Conference on, pages 3399-3403. IEEE, 2016.

[509] M. Shehata, F. Khalifa, A. Soliman, A. Takieldeen, M. A. El-Ghar, A. Shaffie, A. C. Dwyer, R. Ouseph, A. El-Baz, and R. Keynton. 3d diffusion mri-based cad system for early diagnosis of acute renal rejection. In Biomedical Imaging (ISBI), 2016 IEEE 13th International Symposium on, pages 1177-1180. IEEE, 2016. 
[510] F. Khalifa, A. Soliman, A. Takieldeen, M. Shehata, M. Mostapha, A. Shaffie, R. Ouseph, A. Elmaghraby, and A. El-Baz. Kidney segmentation from ct images using a 3d nmf-guided active contour model. In Biomedical Imaging (ISBI), 2016 IEEE 13th International Symposium on, pages 432-435. IEEE, 2016.

[511] F. Khalifa, A. El-Baz, G. Gimel'farb, and M. A. El-Ghar. Non-invasive image-based approach for early detection of acute renal rejection. In Proc. $13^{\text {th }}$ Intern. Conf. on Medical Image Computing and Computer-Assisted Intervention (MICCAI 2010), pages 10-18, Beijing, China, September 20-24, 2010.

[512] F. Khalifa, G. M. Beache, M. Abou El-Ghar, T. El-Diasty, G. Gimel'farb, M. Kong, and A. El-Baz. Dynamic contrast-enhanced MRI-based early detection of acute renal transplant rejection. IEEE Trans. Med. Imaging, 32(10):1910-1927, 2013.

[513] F. Khalifa, M. A. El-Ghar, B. Abdollahi, H. Frieboes, T. El-Diasty, and A. El-Baz. A comprehensive non-invasive framework for automated evaluation of acute renal transplant rejection using DCE-MRI. NMR in Biomedicine, 26(11):1460-1470, 2013.

[514] M. Shehata, F. Khalifa, A. Soliman, A. Takieldeen, M. Abou El-Ghar, A. Shaffie, A. C. Dwyer, R. Ouseph, A. El-Baz, and K. Robert. 3D diffusion MRI-based CAD system for early diagnosis of acute renal rejection. In Proceedings of IEEE 13th International Symposium on Biomedical Imaging, (ISBI'16), pages 1177-1180. IEEE, 2016.

[515] M. Shehata, F. Khalifa, E. Hollis, A. Soliman, E. Hosseini-Asl, M. Abou El-Ghar, M. El-Baz, A. Dwyer, A. El-Baz, and R. Keynton. A new non-invasive approach for early classification of renal rejection types using diffusion-weighted MRI. In Proceedings of IEEE 23rd International Conference on Image Processing, (ICIP'16), page (In Press). IEEE, 2016.

[516] F. Khalifa, M. Abou El-Ghar, B. Abdollahi, H. B. Frieboes, T. El-Diasty, and A. ElBaz. A comprehensive non-invasive framework for automated evaluation of acute renal transplant rejection using DCE-MRI. NMR in Biomedicine, 26(11):1460-1470, 2013.

[517] M. Shehata, F. Khalifa, A. Soliman, A. E.-G. Mohamed, D. Amy, G. Gimel'farb, R. Keynton, and A. El-Baz. A promissing non-invasive CAD system for kidney function assesment. In Proceedings of 19th Medical Image Computing and Computer Assisted Intervention, (MICCAI'16), page (In Press), Athens, Greece, October 17-21, 2016.

[518] A. Farag, A. El-Baz, S. Yuksel, M. A. El-Ghar, and T. Eldiasty. A framework for the detection of acute rejection with dynamic contrast enhanced magnetic resonance imaging. In Proc. IEEE Intern. Symp. on Biomedical Imaging: From Nano to Macro (ISBI'06), pages 418-421, Arlington, Virginia, USA, April 6-9, 2006.

[519] A. El-Baz, A. Farag, R. Fahmi, S. Yuksel, M. A. El-Ghar, and T. Eldiasty. Image analysis of renal DCE MRI for the detection of acute renal rejection. In Proc. $18^{\text {th }}$ IAPR Intern. Conf. on Pattern Recognition (ICPR-2006), pages 822-825, Hong Kong, China, Aug. 20-24, 2006. 
[520] A. El-Baz, A. A. Farag, R. Fahmi, S. E. Yuksel, W. Miller, M. A. El-Ghar, T. ElDiasty, and M. Ghoneim. A new CAD system for the evaluation of kidney diseases using DCE-MRI. In Proc. $9^{\text {th }}$ Intern. Conf. on Medical Image Computing and Computer-Assisted Intervention (MICCAI-2006), pages 446-453, Copenhagen, Denmark, October 1-6, 2006.

[521] A. El-Baz, A. A. Farag, S. E. Yuksel, M. A. El-Ghar, T. A. Eldiasty, and M. A. Ghoneim. Application of deformable models for the detection of acute renal rejection. In J. S. Suri and A. A. Farag, editors, Deformable Models, volume 1, chapter 10, pages 293-333. Springer, 2007.

[522] A. El-Baz, G. Gimel'farb, and M. A. El-Ghar. New motion correction models for automatic identification of renal transplant rejection. In Proc. $10^{\text {th }}$ Intern. Conf. on Medical Image Computing and Computer-Assisted Intervention (MICCAI-2007), pages 235-243, Brisbane, Australia, Oct. 29 - Nov. 2, 2007.

[523] A. El-Baz, G. Gimel'farb, and M. A. El-Ghar. A novel image analysis approach for accurate identification of acute renal rejection. In Proc. $15^{\text {th }}$ IEEE Intern. Conf. on Image Processing (ICIP-2008), pages 1812-1815, San Diego, CA, USA, Oct. 12-15, 2008.

[524] A. El-Baz, G. Gimel'farb, and M. A. El-Ghar. Image analysis approach for identification of renal transplant rejection. In Proc. $19^{\text {th }}$ IAPR Intern. Conf. on Pattern Recognition (ICPR-2008), pages 1-4, Tampa, FL, USA, Dec. 8-11, 2008.

[525] S. Yuksel, A. El-Baz, A. Farag, M. Abou El-Ghar, T. Eldiasty, and M. Ghoneim. Automatic detection of renal rejection after kidney transplantation. In International Congress Series, volume 1281, pages 773-778. Elsevier, 2005.

[526] M. Abou El-Ghar, A. Farag, T. El-Diasty, A. Shokeir, H. Refaie, Y. Osman, T. Mohsen, and M. Ghoneim. Computer aided detection of acute renal allograft dysfunction using dynamic contrast enhanced MRI. The Egyptian Journal of Radiology and Nuclear Medicine, 42(3):443-449, 2011.

[527] F. Khalifa, G. Beache, M. Nitzken, G. Gimel'farb, G. Giridharan, and A. El-Baz. Automatic analysis of left ventricle wall thickness using short-axis cine CMR images. In Proceedings of IEEE Int. Symp. Biomed. Imaging: From Nano to Macro, pages 1306-1309, Chicago, Illinois, USA, 30 March-2 April, 2011.

[528] M. Mostapha, F. Khalifa, A. Alansary, A. Soliman, J. Suri, and A. El-Baz. Computer-aided diagnosis systems for acute renal transplant rejection: Challenges and methodologies. In A. El-Baz, L. Saba, and J. Suri, editors, Abdomen and Thoracic Imaging, chapter 1, pages 1-35. Springer, 2014.

[529] F. Khalifa, A. Soliman, G. Gimel'farb, R. Ouseph, A. C. Dwyer, T. El-Diasty, and A. El-Baz. Models and methods for analyzing DCE-MRI: A review. Medical Physics, 41(12):1-32, 2014. 


\section{CURRICULUM VITAE}

\section{Ahmed Soliman Naeem Soliman}

Paul C. Lutz Hall, Room \# 309

BioImaging Laboratory

Department of Bioengineering

University of Louisville, Louisville, KY, USA

E-mail: asnaee01@louisville.edu,

ahmedsoli@gmail.com

Tel: (502) 550-8146

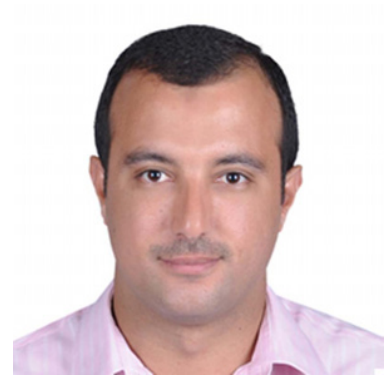

\section{Education}

2016 Ph.D., Electrical Engineering, University of Louisville, Louisville, KY 40292, USA

Ph.D. Dissertation: Computational Methods for the Analysis of Functional 4D-CT Chest Images- Dr. Ayman S. El-Baz and Dr. Jacek M. Zurada, Dissertation Co-advisors.

2010 M.Sc., Computer Engineering and Systems, Mansoura University, Mansoura 35516, Egypt.

M.Sc. Thesis: Schema Integration in Federated Database Systems- Dr. Aly I. Eldesouky, Thesis Advisors.

2004 B.Sc., Computer Engineering and Systems, Mansoura University, Mansoura 35516, Egypt.

Sr. Project: Complete Wireless Robot vision navigation System.

\section{Experience}

2012-2016 Graduate Research Assistant, BioImaging laboratory, Department of Bioengineering, University of Louisville, Louisville, KY 40292, USA.

2005-2011 Graduate Research Assistant, Department of Computer Engineering and Systems, Mansoura University, Mansoura 35516, Egypt.

\section{Professional Affiliations and Training}


- Member, Institute of Electrical and Electronics Engineers (IEEE).

- Member, IEEE Signal Processing (SPS) Society.

- Member, Egyptian Engineers Syndicate, Electrical Engineering.

\section{Awards and Recognition}

- Graduate Dean's Citation Prize in recognition of excellent achievement as a candidate for advanced degree in the University of Louisville, December 2016.

- Research!Louisville 2016 winner among master and Doctoral Engineering graduate students October 2016.

- IEEE SPS Travel Award 2016 to attend the IEEE International Conference on Image Processing 2016 (ICIP'16).

- Best paper runner-up in the International Symposium on Computational Models for Life Science, November 2013.

- Outstanding Graduate Student in the Electrical and Computer Engineering Department, Speed School of Engineering, University of Louisville, April 2014.

- Theobald Scholarship Award in the Electrical and Computer Engineering Department, Speed School of Engineering, University of Louisville, April 2014.

- University of Louisville Travel Awards in 2013 and 2016.

- NSF Travel Award 2013 to attend the IEEE International Symposium on Biomedical Imaging: From Nano to Macro 2013 (ISBI'13).

- Sponsored Research Tuition Award from Spring 2012 through Fall 2016.

- Ranked first, Undergraduate class 2004, B.Sc. (Honor degree), Mansoura University. Mansoura, Egypt.

- Collage excellence award, that is gifted to distinctive students, during all the five undergraduate years (1999-2004).

- Dakahlia Governorate award, for excellence outstanding students (Sept. 2004).

- Prof. Ashor award, for excellence outstanding students (Sept. 2004).

- Reviewer, Computer Methods and Programs in Biomedicine, International Journal of Biomedical Imaging, Computers in Biology and Medicine.

\section{Class Work Grade}

A total of 36 credit hours in Electrical Engineering, Mathematics, and Computer Science subjects- cumulative GPA is 3.983. Particular course concentration has been in statistical modeling, medical image analysis, and machine learning. Relevant courses include: medical image analysis, computer graphics, parallel programming, digital signal processing, and experimental design.

\section{$\underline{\text { Research Activities }}$}

- Image modeling, 2D, 3D and 4D image segmentation and registration, and texture and shape analysis.

- Development of computer-aided diagnostic (CAD) using computed tomography images for early detection of lung cancer. 
- Development of a CAD using 4D computed tomography (4D-CT) images for early detection of lung injury.

- Development of a CAD using dynamic contrast-enhanced MR abdominal images for early detection of acute renal transplant rejection.

- Assisted in grants writing and preparing primary results for the BioImaging Laboratory, University of Louisville, Louisville, KY, USA.

\section{Publications}

During my Ph.D. (Spring 2012- Fall 2016), I have authored or co-authored more than 50 technical publications that have appeared in world-renown journals, including IEEE Transactions on Medical Imaging, Medical Physics, IEEE journal of biomedical and health, IEEE Transactions on Biomedical Engineering; Pattern Recognition Letters; book chapters; top-rank international conferences and workshops ,including Medical Image Computing and Computer-Assisted Intervention (MICCAI), International Symposium on Biomedical Imaging (ISBI), International Conference on Image Processing (ICIP); and abstracts.

\section{- Journal Articles $($ Total = 12)}

1. A. El-Baz, A. Elnakib, F. Khalifa, M. Abou El-Ghar,P. McClure, , A. Soliman, G. Gimel'farb, "Precise Segmentation of 3D Magnetic Resonance Angiography," IEEE Transaction on Biomedical Engineering, vol. 59, no. 7, pp. 20192029, 2012.

2. A. El-Baz, G. M. Beache, G. Gimel'farb, K. Suzuki, K. Okada, A. Elnakib, A. Soliman, and B. Abdollahi "Computer Aided Diagnosis systems for lung cancer: Challenges and methodologies," International Journal of Biomedical Imaging, vol. 2013, 2013.

3. A. Alansary, A. Soliman, F. Khalifa, A. Elnakib, M. Mostapha, W. Nitzken, M. Casanova, A. El-Baz, "MAP-based framework for segmentation of MR brain images based on visual appearance and prior shape," MIDAS Journal, vol. 1, no. 1, pp. 1-13, 2013 [Online]. Available: http://hdl.handle.net/10380/3440.

4. A. Rudra, A. Chowdhury, A. Elnakib, F. Khalifa, A. Soliman, G. Beache, and A. El-Baz "Kidney Segmentation using Graph Cuts and Pixel Connectivity," Pattern Recognition Letters, vol. 34, no. 13, pp. 1470-1475, 2013.

5. M. Casanova, A. El-Baz, S. Kamat, B. Dombroski, F. Khalifa, A. Elnakib, A. Soliman, A. Allison-McNutt, and A. Switala "Focal cortical Dysplasias in Autism Spectrum Disorders," Acta Neuropathologica Communications, vol. 1, no. 1, pp. 1-11, 2013.

6. H. Sliman, F. Khalifa, A. Elnakib, A. Soliman, G. Beache, A. Elmaghraby, G. Gimel'farb, and A. El-Baz "Myocardial Borders Segmentation from Cine MR Images using Bi-Directional Coupled Parametric Deformable Models," Medical Physics, vol. 40, no. 9, pp. 092302, 2013.

7. A. Elnakib, A. Soliman, M. Nitzken, M. Casanova, G. Gimel'farb, and A. ElBaz "Magnetic Resonance Imaging Findings for Dyslexia: A Review," Journal of Biomedical Nanotechnology, vol. 10, no. 10, pp. 2778-2805, 2014.

8. P. McClure, F. Khalifa, A. Soliman, M. Abou El-Ghar, G. Gimel'farb, A. El- 
magraby, and A. El-Baz "A novel NMF guided level-set for DWI prostate segmentation," Journal of Computer Science and System Biology, vol. 7, no. 6, pp. 209-216, 2014.

9. P. McClure, A. Elnakib, M. Abou El-Ghar, F. Khalifa, A. Soliman, T. ElDiasty, J. S. Suri, A. Elmaghraby, and A. El-Baz "In-vitro and in-vivo diagnostic techniques for prostate cancer: A review," Journal of Biomedical Nanotechnology, vol. 10, no. 10, pp. 2747-2777, 2014.

10. F. Khalifa, A. Soliman, A. El-Baz, M. Abou El-Ghar, T. El-Diasty, G. Gimel'farb, R. Ouseph, and A. Dwyer "Models and Methods for Analyzing DCE-MRI: A Review," Medical Physics, vol. 41, no. 12, pp. 124301, 2014.

11. A. Alansary, M. Ismail, A. Soliman, F. Khalifa, M. Nitzken, A. Elnakib, M. Mostapha, A. Black, K. Stinebruner, M. Casanova, J. Zurada, and A. ElBaz "Infant Brain Extraction in T1-weighted MR Images using BET and Refinement using LCDG and MGRF Models," IEEE Journal of biomedical and health informatics, vol. 20, no. 3, pp. 925-935, 2016.

12. A. Soliman, F. Khalifa, A. Elnakib, N. Dunlap, B. Wang, G. Gimelfarb, R. Keynton, and A. El-Baz "Accurate Lungs Segmentation on CT Chest Images by Adaptive Appearance-Guided Shape Modeling," IEEE Transactions on Medical Imaging,, 2016. Doi = “http://dx.doi.org/10.1109/TMI.2016.2606370”.

\section{- Book Chapters (Total = 4)}

1. A. Soliman, M. Ismail, A. Taki Eldeen, G. Gimel'farb, A. El-Baz, and R. Keynton, "Robust image segmentation with a parametric deformable model using learned shape priors," In: Biomedical Image Segmentation: Advances and Trends, A. El-Baz, X. Jiang, and J. Suri, Eds, Taylor \& Francis, 2016, ch. 3, pp. 75-96, ISBN-13: 978-1482258554.

2. A. Soliman, P. McClure, F. Khalifa, A. Taki Eldeen, M. Abou El-Ghar, T. Eldiasty, A. Elmaghraby, A. El-Baz, "Novel NMF-based CAD system for early diagnosis of prostate cancer by using 4-D diffusion weighted magnetic resonance images (DW-MRI)," In: Biomedical Image Segmentation: Advances and Trends, A. El-Baz, X. Jiang, and J. Suri, Eds, Taylor \& Francis, 2016, ch. 14, pp. 309-340, ISBN-13: 978-1482258554.

3. M. Shehata, F. Khalifa, A. Soliman, A. Taki Eldeen, M. Abou El-Ghar, T. Eldiasty, A. El-Baz, and R. Keynton, "An Appearance-guided deformable model for 4D kidney segmentation using diffusion MRI," In: Biomedical Image Segmentation: Advances and Trends, A. El-Baz, X. Jiang, and J. Suri, Eds, Taylor \& Francis, 2016, ch. 12, pp. 269-288, ISBN-13: 978-1482258554.

4. M. Mostapha, F. Khalifa, A. Alansary, A. Soliman, J. Suri, and A. El-Baz, "Computer aided diagnosis systems for acute renal transplant rejection: Challenges and methodologies," Handbook Abdomen and Thoracic Imaging: An Engineering and Clinical Perspective, A. El-Baz, Luca Saba, and J. Suri Eds, Springer-Verlag, New York, 2014, ch. 1, pp. 1-36. 


\section{- Peer-Reviewed Conference Proceedings $($ Total $=28)$}

1. A. El-Baz, A. Soliman, P. McClure, G. Gimel'farb, M. Abo El-Ghar and R. Falk, "Early Assessment of Malignant Lung Nodules Based on The Spatial Analysis of Detected Lung Nodules," In: Proceedings of IEEE International Symposium on Biomedical Imaging: From Nano to Macro (ISBI'12), Barcelona, Spain, May 2-5, 2012, pp. 1463-1466.

2. B. Abdollahi, A. Soliman, C. Civelek, X. Li, G. Gimel' farb, and A. El-Baz, 'A Novel Gaussian Scale-Space Based-Joint MGRF Framework for Precise Lung Segmentation," In: Proc. IEEE International Conference on Image Processing (ICIP'12), Orlando, Florida, USA, September 30-October 3, 2012, pp. 20292032.

3. A. El-Baz, F. Khalifa, A. Elnakib, M. Nitkzen, A. Soliman, P. McClure, G. Gimel'farb, and M. Abou El-Ghar, "A Novel Approach for global lung registration using 3D Markov Gibbs appearance model," In: Proc. International Conference on Medical Image Computing and Computer-Assisted Intervention (MICCAI'12), Nice, France, October 1-5, 2012, pp. 114-121.

4. B. Abdollahi, A. Soliman, A. C. Civelek, X. F. Li, G. Gimel'farb, and A. ElBaz, "A Novel 3D Joint MGRF Framework for Precise Lung Segmentation," In: Proc. Machine Learning in Medical Imaging(MLMI'12), Nice, France, October 1-5, 2012, pp. 86-93.

5. A. Soliman, F. Khalifa, A. Alansary, G. Gimel'farb and A. El-Baz, "Segmentation of Lung Region Based on Using Parallel Implementation of Joint MGRF: Validation on 3D Realistic Lung Phantoms," In: Proc. IEEE International Symposium on Biomedical Imaging: From Nano to Macro (ISBI'13), San Francisco, CA, April 7-11, 2013, pp. 864-867.

6. H. Sliman, F. Khalifa, A. Elnakib, A. Soliman, G. M. Beache, A. Elmaghraby, and A. El-Baz, "A New Segmentation-Based Tracking Framework For Extracting The Left Ventricle Cavity From Cine Cardiac MRI," In: Proc. IEEE International Conference on Image Processing (ICIP'13), Melbourne, Australia, September 15-18, pp. 689-689. 2013. (Selected for oral presentation).

7. M. Mostapha, A. Alansary, A. Soliman, F. Khalifa, M. Nitzken, M. Casanova, A. El-Baz, "Atlas-Based Approach for the Segmentation of Infant DTI MR Brain Images," In: Proceedings of IEEE International Symposium on Biomedical Imaging: From Nano to Macro (ISBI'14), Beijing, China, April 28-May 2, 2014, pp. 1255-1259.

8. A. Alansary, A. Soliman, M. Nitzken, F. Khalifa, A. Elnakib, M. Mostapha, M. Casanova, and A. El-Baz, "An integrated geometrical and stochastic approach for accurate infant brain extraction," In: Proc. IEEE International Conference on Image Processing (ICIP'14), Paris, France, October 27-30, 2014, pp. 35423546.

9. M. Mostapha, A. Soliman, F. Khalifa, A. Elnakib, A. Alansary, M. Nitzken, M. Casanova, and A. El-Baz, "A statistical framework for the classification of infant DT images," In: Proc. IEEE International Conference on Image Processing (ICIP'14), Paris, France, October 27-30, 2014, pp. 2222-2226. 
10. H. Sliman, A. Elnakib, G. Beach, A. Soliman, F. Khalifa, G. Gimel'farb, A. Elmaghaby, and A. El-Baz, "A novel 4D PDE-based approach for accurate assessment of myocardium function using cine cardiac magnetic resonance images," In: Proc. IEEE International Conference on Image Processing (ICIP'14), Paris, France, October 27-30, 2014, pp. 3537-3541.

11. M. Shehata, F. Khalifa, A. Soliman, R. Elrefai, M. Abou El-Ghar, A. C. Dewyer, R. Ouseph, and A. El-Baz, "A novel framework for automatic segmentation of kidney from DW-MRI," In: Proceedings of International Symposium on Biomedical Imaging: From Nano to Macro (ISBI'15), Brooklyn, New York, April 16-19, 2015, pp. 951-954.

12. A. Soliman, A. Elnakib, F. Khalifa, M. Abou El-Ghar, and A. El-Baz, "Segmentation of pathological lungs from CT chest images," In: Proc. IEEE International Conference on Image Processing (ICIP'15), Quebec City, Canada, September 27-30, 2015, pp. 3655-3659. (Selected for oral presentation).

13. M. Shehata, F. Khalifa, A. Soliman, R. Elrefai, M. Abou El-Ghar, A. Dwyer, R. Ouseph, and A. El-Baz, "A level set-based framework for 3D kidney segmentation from diffusion MR images," In: Proceedings of IEEE International Conference on Image Processing (ICIP'15), Qubec City, Canada, September 27-30, 2015, pp. 4441-4445.

14. M. Ismail, M. Mostapha, A. Soliman, M. Nitzken, F. Khalifa, A. Elnakib, G. Gimel'farb, M. F. Casanova, and A. El-Baz, "Segmentation of infant brain MR images based on adaptive shape prior and higher-order MGRF," In: Proceedings of IEEE International Conference on Image Processing (ICIP'15), Qubec City, Canada, September 27-30, 2015, pp. 4327-4331.

15. A. Soliman, N. Liu, G. Gimel'fatb, and A. El-Baz, "Segmenting Kidney DCEMRI Using 1st-Order Shape and 5th-Order Appearance Priors," In: Proceedings of Medical Image Computing and Computer-Assisted Intervention (MICCAI'15), Munich, Germany, October 5-9, 2015, pp. 77-84.

16. A. Soliman, F. Khalifa, M. Abou El-Ghar, and A. El-Baz, "An ISO-surface based local deformation handling framework of lung tissues," In: Proceedings of IEEE International Symposium on Biomedical Imaging: From Nano to Macro (ISBI'16), Prague, Czech Republic, April 13-16, 2016, pp. 1253-1256.

17. A. Soliman, F. Khalifa, A. Shaffie, N. Dunlap, B. Wang, A. Elmaghraby, and A. El-Baz, "Detection of lung injury using 4D-CT chest images," In: Proceedings of IEEE International Symposium on Biomedical Imaging: From Nano to Macro (ISBI'16), ), Prague, Czech Republic, April 13-16, 2016, pp. 12741277.

18. M. Shehata, F. Khalifa, A. Soliman, A. Takieldeen, M. Abou El-Ghar, A. C. Dewyer, R. Ouseph, A. El-Baz, and R. Keynton, "3D diffusion MRI-based CAD system for early diagnosis of acute renal rejection," In: Proceedings of IEEE International Symposium on Biomedical Imaging: From Nano to Macro (ISBI'16), Prague, Czech Republic, April 13-16, 2016, pp. 1177-1180.

19. M. Ismail, A. Soliman, A. ElTanboly, A. Switala, M. Mahmoud, F. Khalifa, G. Gimel'farb, M. F. Casanova, R. Keynton, and A. El-Baz, "Detection of white matter abnormalities in MR brian images for diagnosis of Autism in children," 
In: Proceedings of IEEE International Symposium on Biomedical Imaging: From Nano to Macro (ISBI'16), Prague, Czech Republic, April 13-16, 2016, pp. 6-9.

20. F. Khalifa, A. Soliman, A. Takieldeen, M. Shehata, M. Mostapha, A. Shaffie, R. Ouseph, A. Elmaghraby, and A. El-Baz, "Kidney segmentation from CT images using a 3D-NMF-guided active contour," In: Proceedings of IEEE International Symposium on Biomedical Imaging: From Nano to Macro (ISBI'16), Prague, Czech Republic, April 13-16, 2016, pp. 432-435.

21. A. Soliman, F. Khalifa, A. Shaffie, N. Liu, N. Dunlap, B. Wang, A. Elmaghraby, G. Gimel'farb, and A. El-Baz, "Image-Based CAD System for Accurate Identification of Lung Injury," In: Proc. IEEE International Conference on Image Processing (ICIP'16), Phoenix, Arizona, September 25-28, 2016, pp. 121-125. (Selected for oral presentation).

22. F. Khalifa, A. Soliman, A. Dwyer, G. Gimel'farb, and A. El-Baz, "A Random Forest-Based Framework for 3D Kidney Segmentation from Dynamic Contrast-Enhanced CT Images," In: Proc. IEEE International Conference on Image Processing (ICIP'16), Phoenix, Arizona, September 25-28, 2016, pp. 3399-3403.

23. M. Shehata, F. Khalifa, E. Hollis, A. Soliman, E.-H Asl, M. Abou El-Ghar, M. El-Baz, A. C. Dewyer, A. El-Baz, and R. Keynton, "A new non-invasive approach for early classification of renal rejection types using diffusionweighted MRI," In: Proc. IEEE International Conference on Image Processing (ICIP'16), Phoenix, Arizona, September 25-28, 2016.

24. A. El Tanboly, M. Ismail, A. Switala, M. Mahmoud, A. Soliman, T. Neyer, A. Palacio, A. Hadayer, M. El-Azab, S. Schaal, and A. El-Baz, "A novel automatic segmentation of healthy and diseased retinal layers from OCT scans," In: Proc. IEEE International Conference on Image Processing (ICIP'16), Phoenix, Arizona, September 25-28, 2016, pp. 116-120.

25. M. Shehata, F. Khalifa, A. Soliman, M. Abou El-Ghar, A. Dwyer, G. Gimel'fatb, R. Keynton, and A. El-Baz, "A promising non-invasive CAD system for kidney function assessment," In: Proceedings of Medical Image Computing and Computer-Assisted Intervention (MICCAI'16), Athens, Greece, October 17-21, 2016, pp. 1-9.

26. A. Soliman, F. Khalifa, A. Alansary, G. Gimel'farb, and A. El-Baz, "Performance evaluation of an automatic MGRF-based lung segmentation approach," In: Proceedings of International Symposium on Computational Models for Life Science (CMLS'13), Sydney, Australia, November 27-29, 2013, vol. 1559, pp. 323-332.

27. M. Mostapha, F. Khalifa, A. Alansary, A. Soliman, G. Gimel'farb, and A. El-Baz, "Dynamic MRI-Based Computer Aided Diagnostic System for Early Detection of Kidney Transplant Rejection: A Survey," In: Proceedings of International Symposium on Computational Models for Life Science (CMLS'13), Sydney, Australia, November 27-29, 2013, vol. 1559, pp. 297-306.

28. H. Sliman, F. Khalifa, A. Elnakib, A. Soliman, G. M. Beache, G. Gimel'farb, A. Emam, A. Elmaghraby, and A. El-Baz, "Accurate segmentation framework 
for the left ventricle wall from cardiac cine MRI," In: Proceedings of International Symposium on Computational Models for Life Science (CMLS'13), Sydney, Australia, November 27-29, vol. 1559, pp. 287-296. (Best paper runner-up).

\section{- Patents and Disclosures (Total $=2$ )}

1. A. El-Baz, A. Alansary, A. Soliman, M. Nitzken, and M. Casanova, "Brain Segmentation Method for Young Children and Adults," US Provisional Patent Application, In submission with UofL OTT, 2013.

2. A. El-Baz, A. Dwyer, R. Ouseph, F. Khalifa, A. Soliman, and M. Shehata, "Computer aided diagnostic system for classifying kidneys," US NonProvisional Patent Application \# 14/676,111.

\section{Graduate Advisors}

Dr. Ayman El-Baz, Dissertation Advisor, Department of Bioengineering; and Dr. Jacek Zurada, Dissertation Co-advisor, Electrical and Computer Engineering Department, Speed School of Engineering, University of Louisville, Louisville, KY. 